\section{MULTIDISCIPLINARY TEAMS: MYTH OR REALITY?}

Rhonda Hawley, Department of Clinical Nursing, University of Sydney

Background: With increasing technology and specialisation patient management in asthma cannot be provided by one clinician or discipline alone. Yet physicians and nurses have a long history of examining patient issues separately. The view is taken that multidisciplinary health care teams that recognise the complementary role of each person in the team have the potential not only to improve patient outcomes and decrease direct institutional health care costs over time but also to increase the quality of patient care. The purpose of this study was to identify the similarities and differences in the work practices especialiy those related to patient education between the clinical nurse consultant (CNC) and the respiratory physician. Method: An ethnographic approach was employed for this study. Data were collected over a 30 month period. Data collection techniques involved field observation and in-depth interviews with 35 participants (CNC's, patients and physicians). Data collection, analysis and interpretation was cyclical and utilised a funnel structure, that is, it had a broad exploratory beginning and became more directed and focused over time. Results and discussion: This study found that although many similarities were identified in the work patterns of the CNC and physicians, distinct differences were also identified particularly in the approach taken to the patient. These differences complemented rather than reinforced or reiterated the approach taken by the physician. Conclusion: From this study it appeared that the question is not one relating to multidisciplinary teams: myth or reality? Rather it is one that focuses on how to acknowledge the contributions of each discipline in the asthma management team.

\section{HEALTH INFORMATION SYSTEM FOR ASTHMA}

Clint Moloney', lan Yang'2, Paul Zimmerman'2, Kwun Fong'2, John McCarthy² for the Asthma Clinical Management Team.

${ }^{1}$ CNC Thoracic and ${ }^{2}$ Division of Thoracic Medicine. The Prince Charles Hospital. Brisbane QLD 4032

Aims: To establish a Health Information System capturing key asthma data focusing on ACHS Clinical Indicators for asthma, clinical information and demographics. Methods: The Asthma Hospital Episode System [AHES] Too (Filemaker Pro V3.0), was developed in consultation with key personnel and trialed in the clinical setting. To ensure co-operation \& ownership, all medical officers offered suggestions. A single form data sheet for E.R. episodes replaced chart entry. Results: In April/May 199932 patients were admitted with DRG codes of asthma. 28 were captured using the AHES tool, meeting $100 \%$ of Indicators 8.1 (severity assessment) \& 8.2 (ongoing assessment). Of 4 charts not using this tool $50 \%$ met 8.1 and $75 \%$ met 8.2 .

\begin{tabular}{|l|l|l|}
\hline KEY DATA April/May 1999 & Female & Male \\
\hline Total Number [AHES] & 18 & 10 \\
\hline Mean age & 37 & 46 \\
\hline Mean FEV1/FVC presentation & $0.96 / 1.4$ & $1.12 / 1.9$ \\
\hline AV LOS = 5.4 days & \multicolumn{2}{|l}{} \\
\cline { 2 - 3 }
\end{tabular}

It is estimated that $>\mathbf{2 0 0}$ data entries will have been collected by March 2000. Conclusion: Successful use of this system clearly shows it is possible to meet $100 \%$ of Indicators 8.1 \& 8.2. As yet computerised discharge planning is not included and Indicator 8.3 is not addressed, however we are addressing this. Ongoing liaison with medical officers will ensure adherence with the tool and improve health informatics usage. The utilisation will be enhanced in the near future by converting the form to a scannable format.

Nil Funding Support

Key words: Intormatics, Asthma Hospital Episode System, Clinical Indicators. Nominated for prizes: Respiratory Nurses Prize

\title{
BACK TO THE FUTURE
}

J Ross; Respiratory CNC, Prince of Wales Hospital

C Sargeant; CAP Programme Manager, SESAHS Learning Service

In 1998 the South Eastern Sydney Area Health Service (SESAHS) developed a Respiratory Clinical Accreditation Programme (CAP), a joint venture with the University of Technology Sydney (UTS) to augment their Graduate Diploma in Acute Care Nursing. Due to declining enrolments in both programmes, a 6 month trial Respiratory CAP was conducted during 1999 at the Prince of Wales Hospital as an independent clinical programme. The aims of the programme were to provide registered nurses the opportunity and environment to promote their clinical expertise in mutually agreed upon areas of respiratory nursing competencies. This was achieved through a coordinated and supported workplace programme. Participants engaged in critical reflection of experiences utilising theory and patient centred activities. Consequently the respiratory CAP has now evolved into a 1year-workplace programme, in which the participants gain 2 subject credits with the University of Technology Sydney. The planned date of commencement of the programme is June 2000 . It is the aim of this paper to outline the following issues: Evolution, structure, benefits and future directions of the programme, as well as the experiences of facilitating the programme at the Prince of Wales Hospital.

\section{AMBULATORY INTRAVENOUS THERAPY IN CYSTIC FIBROSIS A UNIQUE APPROACH}

R. Starkey RN, K. Wild RN, S. Monroe RN.

Respiratory Unit, John Hunter Hospital, Newcastle, NSW.

Ambulatory care of patients with cystic fibrosis has become an increasingly important mode of treatment delivery in recent years. Achieving a safe, efficient, personalized service with a high level of patient satisfaction is a challenge being faced by healthcare providers across Australia. Our ambulatory intravenous antibiotic service has had $\mathbf{5 0}$ cystic fibrosis admissions representing 862 patient days since 1996. The average length of stay is 17.2 days, range $4-44$ days, average age 22years, range $9-40$ years. Most patients have drugs delivered via peripherally inserted central catheter $(54 \%)$ and port-a-cath (38\%). Many patients are treated with dual antibiotic therapy. The most frequently used drugs being Ceftazidime $(50 \%)$, Tobramycin (44\%). Most patients receive 24 hour continuous infusions; other treatment modes include bolus injection and intermittent infusions. Clinical response is measured by weight change and lung function. Outcomes and complications are carefully evaluated. An advantage of our approach is that intravenous therapy continues until the desired clinical response is achieved Although initially reluctant to participate the cystic fibrosis population in the Hunter region has since embraced the service enthusiastically. 
VIRAL INFECTION INDUCES IL-8 MEDIATED EOSINOPHIL ACTIVATION AND NEUTROPHIL INFLUX IN ACUTE ASTHMA.

PAB Wark ${ }^{1}$, J Simpson ${ }^{1}$, MJ Hensley ${ }^{1}$, SL Johnston ${ }^{2}$ I Moric ${ }^{2}$ PG Gibson'1. 1.Airways Reesearch Centre Newcastle, Aust.

2.University of Southampton, UK

Acute exacerbations of asthma are frequently caused by viral infections and remain an increasing cause of hospital admission. The inflammatory mechanisms in viral-induced asthma are poorly understood. Aims: To characterise the pattern of airway inflammation associated with viral induced asthma. Methods: Adults $(n=59)$ presenting to John Hunter emergency room with acute asthma were examined for infection by: serology to influenza (Flu) $A$ and $B$, mycoplasma pneumoniae, legionella pneumoniae; sputum DFA detection for respiratory syncytial virus (RSV); and sputum RTPCR for Flu, RSV, rhinovirus, coronavirus, adenovirus and parainfluenza virus 1 and 3 . Subjects testing positive to one of these were classed as having an infection and acute asthma. Subjects had spirometry and sputum induction on presentation, and were compared to a group with stable moderate-severe asthma ( $n=31$ ) and healthy controls $(n=8)$. Results: Forty five subjects had infection and acute asthma while 14 had acute asthma and no infection. Sputum induction demonstrated those with infection had a higher sputum total cell count $(p<0.001)$, increased neutrophils $(65.5 \%, p<0.001)$ and increased IL-8 (39ng/ml, $p<0.001)$, in comparison to the other groups. Levels of IL-8 correlated with neutrophils $(r=0.7)$. Those with non-infective exacerbations had more eosinophils compared to all groups $(p<0.001)$ and more cells staining positive for IL-5 which correlated with eosinophil numbers $(r=0.4)$. Both groups with exacerbations had elevated sputum eosinophil cationic protein which correlated with total cell count $(r=0.6)$, sputum IL-8 $(r=0.7)$ and eosinophils $(r=0.5)$. Subjects with acute asthma and infection had a longer length of hospital stay (median 2 days), compared to those with non-infective acute asthma $(0, p<0.001)$. Conclusion: Acute severe exacerbations of asthma associated with infection are characterised by sputum neutrophilia, LL- 8 release and eosinophil degranulation. Exacerbations of asthma not associated with infection are characterised by eosinophilic airway inflammation mediated by IL-5. In both cases there is marked eosinophil degranulation and there appears to be an interaction between IL- $B$ mediated inflammation and eosinophilic degranulation.

Funded by Asthma Foundation of New South Wales, Australia.

PERIPHERAL \& CENTRAL CHEMOSENSITIVITY TO CARBON DIOXIDE IS RAISED IN CENTRAL SLEEP APNEA WITH HEART FAILURE.

Solin P, T Roebuck, DP Johns, EH Walters and MT Naughton.

Dept of Respiratory Medicine, Alfred Hospital \& Monash University, Victoria, 3181.

Non-hypercapnic central sleep apnea (CSA) in congestive heart failure (CHF) is due to hyperventilation and hypocapnia, which has been attributed to excessive sensitivity of slowly responding central $\mathrm{CO}_{2}$ chemoreceptors situated in the medulla. However, in a typical 60-second cycle of CSA, three changes of ventilation occur, namely crescendo, decrescendo and apnea. This suggests that more rapidly responsive peripheral chemoreceptors, situated in the carotid body, are involved. Hypothesis: Peripheral chemoreceptor sensitivity is raised in CHF with CSA, and correlates with CSA severity. Methods: Peripheral and central chemosensitivities were measured using McClean's single breath hypercapnic ventilatory response (SBR), and Read's rebreathe hypercapnic ventilatory response (HCVR) under hyperoxic conditions, respectively. Three groups of males were studied: healthy volunteers (Normal), 'idiopathic' CSA patients with LVEF>55\% (N-ICSA), \& stable, severe CHF (LVEF $<45 \%)$. CHF patients were divided into 3 groups based on the absence (CHF-N) or presence of sleep apnea (AHI>5, obstructive $=C H F-O S A$, central $=C H F-C S A$ ). Results are mean $\pm \mathrm{SE} \mathrm{L/min} / \mathrm{mmHgCO}$.

Results: Normal ICSA CHF-N CHF-OSA CHF-CSA p $\begin{array}{lcccccc}\text { no. } & 8 & 11 & 12 & 8 & 12 & \text { (anova) }\end{array}$

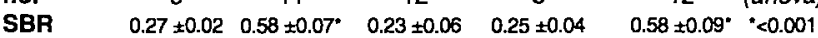
$\begin{array}{lllllll}\text { HCVR } & 2.2 \pm 0.2 & 3.5 \pm 0.3^{*} & 2.0 \pm 0.2 & 1.4 \pm 0.2 & 5.8 \pm 1.2^{*} & <<0.001\end{array}$ In CHF, peripheral chemosensitivity (SBR) correlated with $\mathrm{AHI}(r=0.58, p=$ 0.003 ) and the percentage of central to total apneas $(r=0.64, p=0.001)$, whereas central chemosensitivity (HCVR) correlated inversely with awake $\mathrm{PaCO}_{2}(r=-0.67, p<0.001)$, and less so with AHI $(r=0.435, p=0.030)$. Conclusions: In CHF, peripheral chemosensitivity is directly related to CSA severity, whereas central chemosensitivity is more related to background carbon dioxide. It is therefore likely that elevated central chemosensitivity predisposes to CSA, yet the periodicity is determined by elevated, rapidly responsive peripheral chemosensitivity.

Support: NHMRC, Viertal Foundation, ALF

Key words: central apnea, heart failure, chemosensitivity, ventilatory response Nomination for Awards: John Read Prize
2-METHOXYESTRADIOL INHIBITS EOSINOPHILIA AND AIRWAY HYPERRESPONSIVENESS IN A MURINE MODEL OF ALLERGIC BRONCHOCONSTRICTION

R Vlahos, DJ Fernandes, TL Bamford, T Harris \& AG Stewart Department of Pharmacology, University of Melbourne. Victoria 3010

\section{Airway wall remodelling (AWR) contributes to both airway}

hyperresponsiveness (AHR) and the progressive development of fixed ainway obstruction. We have previously reported that the anti-angiogenic estradiol metabolite, 2-methoxyestradiol (2-MEO) inhibits mitogen-stimulated DNA synthesis and proliferation of human cultured aiway smooth muscle (AJRCCM 159, A531, 1999) raising the possibility that this compound may inhibit smooth muscle hyperplasia, a key component of AWR. Aim: To examine the effects of 2-MEO on the development of bronchoalveolar lavage fluid (BALF) eosinophilia and AHR in a murine model of allergic bronchoconstriction. Methods: Male C57BL/6 mice were sensitised by intraperitoneal (ip) injection of ovalbumin (OVA) on days 0 and 12, followed by 8 aerosol challenges ( $30 \mathrm{~min}$ ) with a combination of OVA $(5 \% \mathrm{w} / \mathrm{v})$ and fetal calf serum (FCS, $5 \% \mathrm{v} / \mathrm{v}$ ) on days $20-27.2-\mathrm{MEO}(0.5-50 \mathrm{mg} / \mathrm{kg})$ was administered daily by ip injection at least $1 \mathrm{~h}$ before OVAFCS challenge. Control mice were challenged with saline and received vehicle $(3 \mathrm{ml} / \mathrm{kg}, 4 \%$ DMSO $+96 \%$ peanut oil) by ip injection. On days 19 and 27 , aiway responsiveness to aerosolised methacholine ( $\mathrm{MCh}, 3-12 \mathrm{mg} / \mathrm{ml})$ was measured using non-invasive, whole body plethysmography (Penh). On day 28 , the mice underwent BAL with sterile saline and cytospot preparations were stained with DiffQuick for eosinophils. Results: There was no difference in MCh reactivity between groups prior to 2-MEO treatment and OVAFCS challenge. 2-MEO dose-dependently reduced OVAFCS-induced AHR to MCh. Moreover, 2-MEO reduced OVAFCS-induced BALF eosinophilia. Conclusion: These results indicate that 2-MEO reduces AHR and BALF eosinophilia induced by repeated allergen inhalation. 2-MEO displays several properties desirable in an agent that targets aiwway wall remodelling.

Supported by Amrad Operations Pty Ltd Key words: Airways hyperresponsiveness, 2-methoxyestradiol, eosinophilia

IMPROVING THE DIAGNOSIS OF OBSTRUCTIVE SLEEP DISORDERED BREATHING IN INFANTS BY INCORPORATING ESOPHAGEAL MANOMETRY INTO CONVENTIONAL POLYSOMNOGRAPHY. V. Muscat 1,2, D. Kennedy2, Y. Pamula2, D. Parsons², G.P. Davidson ${ }^{3}$, T. Omari3, D.L. Moores, J. Martin², P. Buckley'.

'Dept. of Physiology, University of Adelaide, ${ }^{2}$ Dept. of Pulmonary Medicine, Women's and Children's Hospital, Adelaide and ${ }^{3}$ Dept. of Gastroenterology. Women's and Children's Hospital, Adelaide.

Obstructive sleep disordered breathing (OSDB) is currently diagnosed in infants using overnight polysomnography (PSG). Due to 'central - like' obstructive events and normal paradoxical breathing in this population, the accuracy of overnight PSG is unknown. Methods: Nine infants (1-18 months) underwent an overnight PSG with the addition of a manometric pressure-sensing catheter. Respiratory events were identified using both the conventional and the manometric techniques. The proportion of agreement and disagreement between the two methods was calculated for the total events scored and for the individual event types. The composition of missed events was investigated and the respiratory effort present with obstructive apneas, hypopneas and episodes of increased upper airway resistance (UAR) deduced. Results: Conventional PSG agreed with the more accurate manometric PSG scoring for only $47 \%$ of events. Hypopneas had the highest disagreement rate $(66 \%)$ when compared to other respiratory events $(p<0.01)$ and central apneas the lowest $(18 \%, p<0.0001)$. Of events missed by conventional analysis, $90 \%$ were periods of increased UAR. The respiratory effort associated with hypopneas, obstructive apneas and increased UAR was the same for all events $(p>0.1)$. Conclusions: Conventional PSG is routinely used to diagnose OSDB in infants. This study supports that esophageal manometry is a more sensitive indicator of respiratory effort in infants. The data suggest that the incorporation of esophageal manometry into current PSG scoring procedures would substantially improve our ability to diagnose OSDB in the infant population.

Key words: Infants, Esophageal Manometry, Sleep Disorders, Diagnosis. 
TUMOUR NECROSIS FACTOR IS DOWNREGULATED IN ASTHMA AND UPREGULATED BY INHALED CORTICOSTEROID: COMPARATIVE ANALYSIS BY IN SITU HYBRIDISATION, IMMUNOHISTOCHEMISTRY AND COMPETITIVE RT-PCR IN AIRWAY SAMPLES.

Maja Divjak, Eric M. Glare, Michael J. Bailey and E. Haydn Walters Respiratory Medicine. The Alfred Hospital and Department of Medicine. Monash University, Prahran, VIC 3181

Tumour necrosis factor (TNF) is thought to be a mediator of inflammation and fibrosis. Evidence suggests that TNF upregulation occurs in asthmatic subjects, especially those with acute, severe symptoms. Hypothesis: TNF mRNA and/or protein production is upregulated in stable asthmatics compared with normal controls and that inhaled corticosteroids (ICS) downregulate this response. Methods: In situ hybridisation (ISH) and immunohistochemistry (IHC) were performed on airway endobronchial biopsies (EBBx) and bronchoalveolar lavage (BAL) cells from 24 normal controls and 35 stable asthmatics, 22 on ICS. Signal per area in EBBx was measured in the lamina propria and epithelium, with the area of holes subtracted. Competitive(c)RT-PCR was also performed on EBBx from 23 normal controls and 75 stable asthmatics, 62 on ICS and on BAL cells from 30 normal controls and 87 stable asthmatics, 70 on ICS. Results: In EBBX, epithelial TNF was decreased in asthmatics compared with normal with both ISH ( $p \leq 0.0001)$ and IHC $(p=0.002)$. TNF protein was increased in ICStreated asthmatics, compared with asthmatics not on ICS $(\mathrm{IHC}, \mathrm{p}=0.01$ ) and there was a trend towards increasing mRNA expression with ICS treatment (ISH, $\mathrm{p}=0.068$ ). There were no differences in the lamina propria. cRT-PCR also demonstrated decreased TNF mRNA in asthmatics compared with normal ( $p=0.05)$. In BAL, total TNF positive cells and TNF positive macrophages by ISH were again lower in asthmatics compared with normal $\langle p=0.015$ and 0.01 respectively), but there were no differences by IHC or CRT-PCR. Conclusion: Paradoxically, TNF expression was lower in asthmatic airways compared with normal and normalised in the epithelium with ICS. Stable asthmatics may demonstrate impaired TNF expression, whilst 'normal' TNF levels may have a physiologically protective effect.

Supported by Glaxo Wellcome Australia and NHMRC

Key words: tumour necrosis factor, asthma, in situ hybridisation immunohistochemistry, competitive RT-PCR, inhaled corticosteroid

\section{Monday April 10 - Paediatric SIG Orals} $(1330-1530)$

IMPLEMENTATION OF EVIDENCE BASED MANAGEMENT OF ACUTE VIRAL BRONCHIOLITIS

J.U. Barben, C.F. Robertson, P.J. Robinson

Department of Respiratory Medicine. Royal.Children's Hospital. Melbourne

Background: Acute viral bronchiolitis (AVB) is the most common lower respiratory tract infection in the first year of life. Systematic reviews of the literature would suggest that pharmaceutical agents do not influence the course of the disease. Implementation of clinical guidelines is frequently delayed well beyond their dissemination and the publication of clinical evidence. The recently published Australian guidelines for the management of AVB have been evaluated by assessing the current practice of all paediatricians in Australia.

Methods: Questionnaire survey and literature review

Results: From a total of 891 questionnaires $555(62 \%)$ were returned. 373 (67\%) responders treat children with AVB and of these $232(63 \%)$ treat 10 50 children per year. A wide variation in the management practice of $A V B$ was noted. Up to $70 \%$ of paediatricians, who treat AVB, indicated the use of pharmaceutical agents in their outpatient management $(88 \%$ in inpatient management), most only using these agents sometimes or in high-risk children. Paediatric respiratory physicians tended to use bronchodilators and corticosteroids less frequently than the general paediatricians. Compared with many countries in Europe, few Australian paediatricians always use supplementary drugs in the inpatient management of AVB. In particular, bronchodilators $(61 \%$ vs. $6 \%)$ and corticosteroids $(11 \%$ vs. $1 \%)$ are used far less often.

Conclusions: Despite the evidence and the recommendation of the Australian guidelines pharmaceutical agents are used frequently in the management of AVB by paediatricians in Australia. Guidelines alone are not sufficient to implement change and there is a need for more specific strategies to ensure that children receive appropriate management for this common condition.

Key words: acute bronchiolitis, bronchodilators, evidence-based medicine
INHALED FLUTICASONE PROPIONATE (FP) DOES NOT ALTER INFLAMMATION IN THE AIRWAYS OF LUNG ALLOGRAFTS: A DOUBLE BLIND PLACEBO CONTROLLED TRIAL.

H.M. Whithord, B. Orsida, C. Ward, E.H. Walters, T.J. Williams, T. Kotsimbos, \& G.I. Snell, Alfred Hospital and Monash Medical Schooi, Prahran, VICTORIA. Progressive airflow obstruction i.e.: Bronchiolitis Obliterans Syndrome (BOS) remains a major factor limiting long term survival after lung transplantation (LTx). It is likely that inflammation precedes the scarring and remodeling of airways that occurs in this process. Inhaled steroids are widely used in asthma to treat airway inflammation, and have been used post LTX in the setting of early BOS. Methods: 29 stable LTX recipients (LTR) without BOS were recruited 3 to 9 months post $L T$, and randomised to receive either $750 \mu \mathrm{g} \mathrm{FP}$ or an identical appearing placebo. Baseline endobronchial biopsies were taken at routine bronchoscopy at the time of randomisation, and repeated 3 months later. Immunohistochemical staining of the biopsies was performed for $\mathrm{CD} 3$ (pan Tcell), CD8 (T suppressor/cytotoxic), CD4 (T helper/índucer), LCA (leukocyte common antigen) and Neutrophil Elastase. Positive cells were counted per $\mathrm{mm}^{2}$ of subepithelium. Biopsies taken from 10 healthy, nonsmoking volunteers were compared to those of the stable LTR. Ressults: Medians and ranges. 20 paired biopsies suitable for analysis.

\begin{tabular}{|l|l|l|l|l|l|l|}
\hline & No. & $\mathrm{CD} 3 / \mathrm{mm}^{2}$ & $\mathrm{CD8} / \mathrm{mm}^{2}$ & $\mathrm{CD} 4 / \mathrm{mm}^{2}$ & LCA/mm & PMN/mm² \\
\hline $\begin{array}{l}\text { Normal } \\
\text { Controls }\end{array}$ & 10 & $\begin{array}{l}347.5 \\
(155-661)\end{array}$ & $\begin{array}{l}146.1 \\
(64-285)^{* *}\end{array}$ & $\begin{array}{l}235.0 \\
(64-167)\end{array}$ & $\begin{array}{l}567.6 \\
(339-891)\end{array}$ & $\begin{array}{l}124.7 \\
(42-285)\end{array}$ \\
\hline Baseline SLTX & 20 & 425 & 228.2 & 302.6 & 766 & 159.7 \\
& & $(136-2149)$ & $(37-1023)$ & $(49-1621)$ & $(331-5006)$ & $(59-717)$ \\
\hline $3 / 12$ & 9 & 255.0 & 121.6 & 151.3 & 682.0 & 175.3 \\
FP & & $(7-1518)$ & $(7-586)$ & $(10-661)$ & $(77-2391)$ & $(21-624)$ \\
\hline $3 / 12$ & 11 & 501.0 & 199.0 & 405.5 & 733.0 & 239.9 \\
Placebo & & $(133-1835)$ & $(79-1494)$ & $(77-578)$ & $(361-4118)$ & $(81-767)$ \\
\hline
\end{tabular}

${ }^{\star \star} p<0.05$.

CD8 positive cells were increased in the LTR compared to controls, no other significânt differences were detected. No significant differences in any of the cell markers were found after 3 months treatment with FP compared to baseline and placebo. Conclusions: In stable LTR on triple immunosuppression the addition of inhaled FP does not affect the degree of airway inflammation. CD8 positive cells are increased in stable LTR, but neutrophil numbers in the subepithelium are not different from controls. This contrasts with the increased numbers of neutrophils in established BOS. This group of patients is being followed over a longer period to assess whether emergent changes reflect the long-term risk of BOS.

Supported by the NHMRC \& Glaxo Wellcome Australia.

Key words: Lung transplantation, airway inflammation, inhaled steroids

IN-UTERO CIGARETTE SMOKE EXPOSURE AND ALVEOLAR ATTACHMENTS IN THE SUDDEN INFANT DEATH SYNDROME John Elliot,(1) Neil Carroll,(1) Alan James, (1) Philip Robinson.(2) (1) Dept of Pulmonary Physiology. Sir Charles Gairdner Hospital, Perth Western Australia and (2) Dept of Respiratory Medicine. Royal Children's Hospital, Melbourne, Victoria, Australia.

The harmful effects of passive cigarette smoke exposure to infants include increased frequency of asthma exacerbations, increased incidence of lower respiratory viral infections, reduced lung function in the neonatal period, and an increased risk of Sudden Infant Death Syndrome (SIDS). We have previously shown an increase in the thickness of the inner airway wall in infants who have died of SIDS and were exposed to maternal cigarette smoke. To determine the effects of in-utero cigarette smoke exposure on the structure of the lung in infancy, we examined airway dimensions and airway alveolar attachments in $\mathbf{3 1 9}$ airways from 32 infants who died from SIDS. Cases were divided into four groups based on the maternal history of cigarette smoking: no smoke exposure $(n=6)$; post natal smoke exposure only $(n=4)$; in-utero smoke exposure only $(n=4)$; and both in-utero and post natal exposure $(n=18)$. In transverse sections of intra parenchymal airways stained with haematoxylin and eosin, the length of the outer perimeter of the airway was measured and the number of alveolar attachment points was counted. There were no significant differences between the groups with regard to airway size (internal perimeter) or outer airway perimeter. The mean $( \pm \mathrm{SE})$ distance, in millimetres, between alveolar attachments was similar in infants who had no smoke exposure $(0.08 \pm .002)$ and those that had post natal exposure only $(0.08 \pm 0.004)$. This distance was greater in infants exposed to cigarette smoke in-utero $(0.1 \pm .004, p<0.05)$ and in those exposed both in-utero and post natally $(0.09 \pm .002, p<0.05)$. These findings suggest that in-utero cigarette smoke exposure may have significant effects on infant lung structure and may help explain the observed abnormalities of lung function in infants of smoking mothers. Support: the National SIDS Council of Australia and NHMRC.

Key words: In-utero cigarette smoke, Alveolar attachments, SIDS. Awards: nil. 
MEASUREMENTS OF EXHALED NITRIC OXIDE WITH THE SINGLEBREATH TECHNIQUE IN INFANTS.

Peter Franklin1, Stephen Stick²

1Department of Paediatrics, University of Western Australia, 2Department of Respiratory Medicine, Princess Margaret Hospital for Children, WA, 6008. Exhaled nitric oxide levels in adults are flow dependent. We have reported that exhaled nitric oxide (eNO) can be measured in infants using a modified single-breath technique with positive expiratory pressure (1). The aim of this study was to investigate if this modified technique could detect a flow dependence for eNO in infants. Further we compared the single-breath technique with other methods of collecting eNO from infants - bag collections (mixed expired NO) and tidal breathing (peak tidal NO). Methods: Exhaled NO were measured in 6 infants using three different collection methods; (i) single-breath technique using two different expiratory flows ( 10 and $40 \mathrm{ml} / \mathrm{s}$ ), (ii) tidal breathing into a gas sampling bag and (iii) tidal breathing directly into a nitric oxide analyser. All collections were made on the same day while the infant was asleep following a dose of chloral hydrate $(80-100 \mathrm{mg} / \mathrm{kg})$. Results: A significant flow dependence for eNO was demonstrated using the single-breath technique. Average eNO levels were $28.8 \mathrm{ppb}$ with a flow of $10 \mathrm{ml} / \mathrm{s}$ and $20.1 \mathrm{ppb}$ with a flow of $40 \mathrm{ml} / \mathrm{s}$. There were significant correlations between the eNO levels measured by the single-breath technique and both bag collections $(r=0.64)$ and peak tidal NO $(r=0.93)$. Conclusions: A flow dependence for eNO can be demonstrated in infants using single-breath measurements. There is good agreement between eNO levels measured by the single-breath method with levels measured using other techniques.

1. Wildhaber et al. 1999. AJRCCM, 159: 74-78.

Supported by the NH\&MRC

Key words: Exhaled nitric oxide, infants

Nominations for awards: nil
PATIENTS WITH A WRITTEN ASTHMA PLAN HAVE A SHORTER HOSPITAL STAY.

JR Holberton, DA Fitzgerald, DM Cooper, Judy Cordwell \& BF Whitehead. John Hunter Children's Hospital, Newcastle, NSW

Aims: Asthma continues to be a major burden for its sufferers, their farnilies and society in general. We wished to obtain a better understanding of our local population, to see how this compared with other published work and to see if there were areas in which we were deficient or could improve. Methods: A 3 part "PAQART" (paediatric asthma quality assurance review team) questionnaire was administered to all asthma admissions (or their parents/guardians) from 01/04/98 until 30/06/98. This consisted of parental information on background and current asthma status, as well as an admission and a discharge part filled in by medical stafi.

Results: There were 103 admissions with asthma over this time to 98 patients and forms were completed on 79 of them (76.9). Firstly we compared patients without forms (by medical record review) with those who had data and found no significant differences in: age, sex, length of stay, first diagnosis, prior oral steroid use, prior antibiotic use, "PAQART" admission score, other medical problems and those who received specialist medical follow up.

37 of the 68 known asthma patients had a written asthma plan, which was followed by 14 . When these groups were compared there was a significant decrease in the length of stay in those patients who had an asthma plan versus with those without, which was further decreased, though not significantly, if the plan was followed. There was no difference in their age, admission score, background disease as measured by days off school and courses of steroids in the past year, and frequency of wheezing. They had a non-significant increase in pre-hospital oral steroids $(25 \%$ Vs. $12.5 \%$ $P=0.21)$, and a tendency to be girls $(59.5 \%$ Vs. $38.8 \% P=0.09$ ) Discussion: Our study further advances the benefits of having an asthma plan, while raising the question of why in our population boys seem to be less likely than girls to actually possess one.

\section{AIRWAY INFLAMMATION IN CHILDREN WITH INFREQUENT EPISODIC} WHEEZE.

Carolyn Maclennan 1,3 , Paul Hutchinson ${ }^{2}$, Nicholas Freezer ${ }^{1,3}$, Stephen Holdsworth ${ }^{2}$.

(1) Departments of Respiratory Medicine and (2) Clinical Immunology, Monassh Medical Centre and (3) Centre for Heart and Chest Research. Monash University. Melbourne. Australia.

In Australia 1 in 4 children will have at least one episode of wheeze per year. In adults the role of airway inflammation in asthma is well characterised with increased $T$ lymphocytes activation and a bias towards Th2 cells, however, few studies have been performed in children. In this study, a non-bronchoscopic bronchoalveolar lavage (BAL) technique was used to obtain T-cells from the lungs of children with a history of infrequent episodic wheeze (2-12 episodes/yr) and a control group with no history of cough or wheeze. 32 children were studied ( 16 asthmatic, 16 controls) aged 0.5 to 12 years and all children were asymptomatic at the time of study. There was no significant difference in the differential cell count on BAL fluid. Cell surface markers were performed on 12 asthmatics and 15 controls. In both groups the majority of $\mathrm{T}$ cells were $\mathrm{CD} 3, \mathrm{CD} 8$ positive with a CD4:CD8 ratio of 0.6 . No differences were found between the groups in the level of $T$ cell CD25, CD38, CD69 and HLA-DR expression. Intracellular cytokine studies were performed on 9 asthmatics and 6 controls. CD4 T-cell cytokine (IFN $\gamma, \mathrm{IL}-2, \mathrm{IL}-4, \mathrm{IL}-10$ ) production was measured by fiow cytometry following stimulation. No statistically significant difference between the groups was found, with IFN $\gamma$ being the highest in both groups. There was no correlation between total and specific serum IgE and markers of airway inflammation. These results suggest that significant airway inflammation is not present in asymptomatic children with infrequent episodic wheeze. They support the current guidelines for asthma management in Australia that children with infrequent episodic asthma do not require treatment with anti-inflammatory asthma medications between exacerbations of their lung disease.

Key words: asthma, airway inflammation, paediatrics
GREATER AIRWAY SMOOTH MUSCLE CONTENT IN THE LUNGS OF MALE INFANTS

Karen McKay

Children's Chest Research Centre \& Department of Respiratory Medicine. Royal Alexandra Hospital for Children. Westmead. New South Wales, 2145 Growth-related changes in lung structure may have a significant impact upon the normal functioning of the paediatric lung. Male children and adolescents have lower maximal airflow values and hence aiway calibre than females of the same height and age. In addition to these differences in airway function, there is evidence for gender differences in the rate of growth, of large and small aiways in children. Gender differences in small airways structure in infants studied at post-mortem have recently been reported. Such differences have been suggested as part of the pathophysiological basis, of the greater prevalence of lower respiratory tract illness in male children. In this study, standard methodology was used to perform computer-assisted morphometry. The inner $\left(W_{A i}\right)$ and outer $\left(W_{A 0}\right)$ airway wall areas, as well as the area of aiwway smooth muscle $\left(A_{s m}\right)$ and cartilage $\left(A_{c a r}\right)$, were measured in formalin fixed lungs from 7 male and 5 female infants (age: 2-123 days). These children had died suddenly from non-respiratory causes and their parents had donated their lungs for research purposes after coronial investigation. Random effects regression was used to calculate and compare the relationship between the airway internal perimeter $\left(P_{b m}\right)$ and these components in cartilaginous $(n=127$, $\left.P_{b m}: 1.4-19.8 \mathrm{~mm}\right)$ and non-cartilaginous $\left(n=135, P_{b m} ; 0.6-5.0 \mathrm{~mm}\right)$ airways from male and female infants. These relationships, and statistically significant gender differences $(* 0<0.01)$ are shown below.

\begin{tabular}{|l|l|l|l|l|}
\hline & \multicolumn{2}{|c|}{ Non-cartilaginous airways } & \multicolumn{2}{c|}{ Cartilaginous airways } \\
\hline & Female & Male & Female & Male \\
\hline$V W_{A}=$ & $0.098 P_{b m}-0.010$ & $0.099 P_{b m}-0.001$ & $0.063 P_{b m}+0.094$ & $0.076 P_{b m}+0.075^{*}$ \\
\hline$V W_{A 0}=$ & $0.192 P_{b m}-0.044$ & $0.135 P_{b m}+0.032$ & $0.158 P_{b m}+0.170$ & $0.199 P_{b m}+0.041$ \\
\hline$V A_{s m}=$ & $0.054 P_{b m}+0.003$ & $0.056 P_{b m}+0.011^{*}$ & $0.032 P_{b m}+0.064$ & $0.034 P_{b m}+0.087^{*}$ \\
\hline$\sqrt{ } A_{c a t}=$ & - & - & $0.101 P_{b m}-0.107$ & $0.096 P_{b m}-0.100$ \\
\hline
\end{tabular}

Therefore, while airways of the same size in infant male and female lungs have similar adventitial thicknesses, cartilaginous airways in infant males have significantly more tissue luminal to the smooth muscle. There is also significantly more smooth muscle in airways of all sizes from male infants. Airway structure therefore differs in male and female infants, and this greater amount of smooth muscle and thicker inner airway wall, may provide part of the explanation for gender differences in airway function.

Supported by: The Financial Markets Foundation for Children and The New Children's Hospital Teddy Bears Picnic

Key words: airway smooth muscle, airway wall structure, gender, infant 


\section{AEROBIC FITNESS AND PHYSICAL ACTIVITY IN BOYS}

R.G.D. Roberts ${ }^{1}$, M.I. Toohey ${ }^{3}$, S.I. Reid², K. Quang ${ }^{2}$, R.C. Starr ${ }^{2}$ \& J. Kemp ${ }^{3}$

(1) Department of Respiratory Medicine and (2) Hugh Williamson Gait Laboratory, Royal Children's Hospital, and (3) Department of Human Movement, Australian Catholic University, Melbourne, Australia

Aerobic fitness (AF) was estimated in two groups of boys by assessing the distance covered in a 6 minute run (6MD) and was compared with direct measurements of peak aerobic power $\left(\mathrm{vO}_{2}\right)$ in subgroups of these boys. 21 year 8 boys $(14.1 \mathrm{yr}, 1.68 \mathrm{~m}, 56.5 \mathrm{~kg}, \mathrm{BMl}: 20.01, \mathrm{FVC}: 3.8 \mathrm{~L}=94 \%$ pred, $\mathrm{FEV}_{1}: 3.5 \mathrm{~L}=104 \%$ pred $)$ and 31 year 5 boys $(11.0 \mathrm{yr}, 1.47 \mathrm{~m}, 41.7 \mathrm{~kg}$, BMl:19.28, FVC:2.6L=96\%pred, FEV,$: 2.36 \mathrm{~L}=104 \%$ pred) completed a Bruce protocol treadmill run during which breath by breath measurements of metabolic parameters were obtained from a COSMED K4b2. Peak HR was $195 \mathrm{bpm}\left(97 \%\right.$ pred) and RER 1.12 in yr 8 boys while peak vO $\mathrm{O}_{2}(50.8$ $\mathrm{ml} / \mathrm{kg} \cdot \mathrm{min}, 104 \%$ pred) was significantly correlated with $6 \mathrm{MD}(1249 \mathrm{~m}$, $\mathrm{P}=0.015)$ and treadmill run time $(11.7 \mathrm{~min}, \mathrm{P}<0.01)$ but negatively correlated with $B M I(P=0.002)$. The regression equation predicting $\mathrm{VO}_{2}$ from $6 \mathrm{MD}$ was $\mathrm{vO}_{2}=0.0385 \times 6 \mathrm{MD}+2.93\left(\mathrm{R}^{2}=0.287\right)$. In the younger group, peak $\mathrm{HR}$ reached $198 \mathrm{bpm}\left(97 \%\right.$ pred) and RER 1.07 while peak $\mathrm{vO}_{2}(44.7 \mathrm{~m} / \mathrm{kg}$.min $99 \%$ pred) was not significantly correlated with $6 \mathrm{MD}(1071 \mathrm{~m}, \mathrm{P}=0.075)$ or $B M I(P=0.67)$ but was significantly related to treadmill run time $(10.5 \mathrm{~min}$ $P=0.03)$. The 7 day recall Physical Activity (PA) Questionnaire for Children (PAQ-C) was administered to both groups of boys but no significant relationship was seen between PAQ-C score (yr 8:3.025 \& yr 5:3.178) and peak $\mathrm{vO}_{2}(\mathrm{P}=0.36 \& \mathrm{P}=0.90$ respectively). These resuits suggest that $P A$ and $A F$ levels vary with age and population selection and that in confirmation of previous investigations, in the age groups examined AF may be largely independent of PA.

Key words: Boys, peak aerobic power, 6 minute run, physical activity

\section{Monday April 10 - Population Health SIG Orals} $(1330-1530)$

DETERMINANTS OF 'HEALTHY' LUNG FUNCTION IN A REMOTE ABORIGINAL COMMUNITY IN NORTHERN AUSTRALIA

Graeme Maguire1, Stephen McDonald1,2, Norma Benger1 \& Bart Currie 1,2 (1) Menzies School of Health Research, Darwin (2) Flinders University, Royal Danwin Hospital Clinical School

Respiratory disease is a significant cause of morbidity and mortality for Indigenous Australians especially chronic respiratory disease. It is becoming clearer that determinants of respiratory health in non-Aboriginal Australians can not be generalised to Aboriginal Australians. We have commenced a multidisciplinary study of respiratory disease with the aim of optimising preventions, interventions and standards of care for remote Aboriginal communities. Our initial work has focused on a detailed assessment of baseline lung function. Methods: Cross-sectional survey of 202 adult residents (18-80 years) of a remote northern Australian rural Aboriginal community. Spirometry, histamine challenge, respiratory symptoms and signs, and established risk factors for respiratory disease were assessed. Results: Data from $54 \%$ of participants who had no evidence of respiratory disease or significant bronchial hyperreactivity (BHR) were used for analysis. These subjects were those with no recent wheeze, chronic bronchitis, BHR $\left\langle\mathrm{PD}_{20} \mathrm{FEV}_{1}<3.8 \mu \mathrm{mol}\right.$ histamine $)$ or reversibility with salbutamol and unremarkable chest auscultation. Of those without respiratory disease/BHR, $81 \%$ of men and $70 \%$ of women were current or past tobacco smokers. Age, height and gender explained $59 \%$ of the variance in $\mathrm{FEV}_{1}$ and $60 \%$ in $\mathrm{FVC}$. Predicted values for FEV, were $6.3 \%$ (0.3(SEM)) lower and FVC $0.6 \%$ (0.5(SEM)) lower than those predicted by Veale et al[1]. The decline in predicted FEV, and FVC over increasing agé paralleled that of Veale. Conclusion: The 'healthy' spirometry findings in this northern Australian Aboriginal community support the generalisation of the findings of Veale to at least other remote rural populations, with values of FEV, and FVC being lower than those of non-Indigenous Australians. Further studies are required to determine if these values apply to Indigenous Australians in southern and urban centres. We are now planning prevention and intervention programs for chronic respiratory disease in remote communities.

Supported by: AMAIAPMA Aboriginal Health Initiative, Flinders University Northern Territory Clinical School, Community Health and Anti-Tuberculosis Association, CRC for Aboriginal and Tropical Health.

Key words: Indigenous health, respiratory physiology

Nominations for Awards: nil

1. Veale, A., et al., 'Normal' lung function in rural Australian Aborigines. Aust NZ J Med, 1997. 27: p.543-549.
ADRENOCORTICAL SUPPRESSION AND BONE MINERAL DENSITY IN ASTHMATIC CHILDREN TREATED WITH HIGH DOSE FLUTICASONE PROPIONATE

Derrick Sim 1 , David Armstrong ${ }^{1}$, Caroline Clarke², Christine Rodda², Nicholas Freezer 1

1-Department of Paediatric Respiratory Medicine and 2 Department of Paediatric Endocrinology, Monash Medical Centre. Victoria 3162

It is recognised that inhaled corticosteroids have systemic effects on the hypothalamic-pituitary-adrenal (HPA) axis and bone metapolism. Fluticasone propionate $(\mathrm{FP})$ is a high potency corticosteroid with moderate accumulation after multiple dose therapy. The prevalence of HPA axis suppression and low bone mineral density in children treated with high dose fluticasone propionate is investigated in this cross-sectional study. Methods: 45 children and adolescents aged $4-20$ years attending asthma clinics at Monash Medical Centre were enrolled. Criteria for inclusion into the study were subjects treated with $\mathrm{FP}$ at a dose $\geq 500 \mathrm{mcg}$ for at least 6 months using a puffer and spacer or Accuhale $\Gamma^{\mathbb{B}}$ with mouth rinsing. Subjects were excluded if they had received oral or systemic steroids in the previous two weeks or had another chronic illness. Early morning serum cortisol levels were obtained and those subjects with a cortisol level less than 400nmol/ underwent a modified Synacthen test (tetracosactrin $250 \mathrm{mcg} / \mathrm{M}$ ) and dual energy X-ray absorptiometry (DEXA scan) of the lumbar spine and total body. Results: The subjects of mean age 12.8 years (range $4-20$ years) had been treated with mean daily dose FP $979.6 \mathrm{mcg} / \mathrm{m}^{2} /$ day (range $333-2985 \mathrm{mcg} / \mathrm{m}^{2} /$ day) for a mean duration of 2.3 years (range $6-48$ months). $68 \%$ of subjects had serum cortisol levels less than $400 \mathrm{nmol}$. Of these subjects, $25 \%$ demonstrated an inadequate cortisol response to tetracosactrin stimulation, defined as a less than twofold increase in serum cortisol from baseline and a peak cortisol level $\leq 550 \mathrm{nmol} / \mathrm{L}$ at $\leq 60$ minutes post tetracosactrin administration. None of the subjects in the study demonstrated low bone mineral density for bone age $(<-1.5$ SD from mean) when compared to healthy sex and age matched controls. There was a significant negative correlation (correlation coefficient $-0.31, p<0.05$ ) between the dose of FP / body surface area and morning serum cortisol levels. Conclusion: These results indicate that use of high dose inhaled fluticasone propionate may be associated with significant adrenocortical suppression in a dose dependent manner. There was no associated reduction in bone mineral density in this study group.

Key words: adrenocortical suppression, bone mineral density, fluticasone propionate, asthma, paediatrics

DECLINE IN LUNG FUNCTION IN ADULT AUSTRALIANS OF ABORIGINAL DESCENT (AAAD)

Musk AW, James AL, Ryan GF, Mukherjee S, Waring JA, LeSouef PN, McCarthy J, Wood MM, Johnson D, Palmer LJ, de Klerk NH. Perth Respiratory Epidemiology Group, Sir Charles Gairdner Hospital, WA. Background: AAAD are known to have lower levels of lung function than adult Australians of European descent. Our previous studies of crosssectional design have been unable to determine if this results from reduced lung growth during childhood, earlier cessation of lung growth or a greater decline in lung function with increasing age, which may all be due to environmental factors. Aim: To measure the rate of decline in lung function in AAAD and its relation to respiratory symptoms and smoking. Methods: Surveys of respiratory function were carried out in a community of AAAD in the northwest of Western Australia in 1993, 1995 and 1999 to provide data for longitudinal analysis. Subjects answered a modified BMRC questionnaire on cough, sputum, dyspnoea and smoking. FEVI was measured with a dry bellows spirometer. Generalised estimating equations were used to estimate the effect of calendar time on the rate of decline in FEV 1 where people were assumed to be random effects with repeated measures. Fixed effects for age, height and smoking were included using the STATA v5.0 sotware. Results: In the 1999 survey, the prevalence of cigarette smoking was $54.4 \%$ in males and $29.6 \%$ in females. Levels of lung function measured cross-sectionally were similar to those in our previous studies and comparable with those of other studies of AAAD. After adjustment for height and smoking, the average rate of decline in $F E V_{1}$ in adults was estimated to be $31 \mathrm{mls} / \mathrm{yr}(\mathrm{SE}=3 \mathrm{mls} / \mathrm{yr})$ in males and $23 \mathrm{mls} / \mathrm{yr}(\mathrm{SE}=2 \mathrm{mls} / \mathrm{yr}$ ) in females. The presence of doctor diagnosed asthma was associated with an increased decline of $9 \mathrm{mls} / \mathrm{yr}$ ( $\mathrm{SE}=3 \mathrm{mls} / \mathrm{ys})(\mathrm{P}=0.008)$, self-reported wheeze with an increased decline of 5 $\mathrm{mls} / \mathrm{yr}(\mathrm{SE}=2 \mathrm{mls} / \mathrm{ys})(\mathrm{P}=0.05)$ and current cigarette smoking with an increased decline of $9 \mathrm{mls} / \mathrm{yr}$ ( $S E=3 \mathrm{mls} / \mathrm{ys})(P=0.002)$. The presence of cough, sputum, or dyspnoea was not associated with a significant increase in

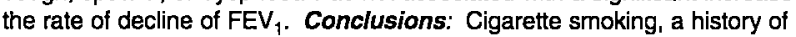
asthma and the presence of wheeze are associated with greater rates of decline of lung function. This provides further evidence that environmental factors may be important in determining the level of impairment of lung function in AAAD. Supported by HDWA \& MEDWA. 
IMPROVEMENTS IN RESPIRATORY HEALTH STATUS \& PREDICTING UTILISATION IN THE SA HEALTHPLUS SOUTHERN RESPIRATORY COORDINATED CARE TRIAL

\section{Pieter Walker, Peter Frith \& Mike Melino,}

Health Systems Development Service, Dept. Human Services, SA.

The Council of Australian Governments (COAG) approved the development of a national Co-ordinated Care trials with the primary aim to show that coordinating patient care will improve health outcomes and reduce demand. AIMS: 1) to test the hypothesis that a significant proportion of health service utilisation variance can be predicted from baseline health-outcome measures, demographics and historical utilisation. 2) to test the hypothesis that coordinated care will produce significant improvements in respiratory health status and quality of life over time. Method: A sample of 225 patients with a diagnosis of COPD from the Southern region of Adelaide were randomly allocated to either the co-ordinated care intervention (I) $(n=145)$ or the control (C) group (standard medical care, $n=80$ ). All patients were administered an SF-36 health status questionnaire, a respiratory disease questionnaire and had their Medical Benefits, Pharmaceutical Benefits Scheme (MBS \& PBS) and hospital in-patient data tracked for 2 years prior and up to 1 year after enrolment. RESULTS: Using multiple regression (stepwise) analysis $40 \%$ of the variance associated with hospital utilisation (as measured by equiseps), $14 \%$ of MBS utilisation and $41 \%$ PBS utilisation could be predicted from a combination of historical utilisation, SF36 scores and the Dyspnoea Impact \& Symptoms Questionnaire (DISQ). There were no significant differences in SF-36 scores over time, however there were significant improvements in intervention compared to control patients over time on self-efficacy, affect, functional and cognitive subdomains of the DISQ using repeated measures analysis of variance $(p<0.05)$. CONCLUSIONS Health risk assessment models in COPD can be developed to predict health service utilisation and can be used to identify patients most at risk of hospitalisation and higher users of medical and pharmaceutical benefits. Furthermore, intervention patients who have received coordinated care for 12 months are beginning to show significant improvements over time in regards aspects of health related quality of life.

\section{6}

LONGITUDINAL VALIDATION OF AN EPIDEMIOLOGICAL DEFINITION OF ASTHMA

Toelle B.G. Marks G.B., Belousova E.B., Xuan W, Peat J.K., Woolcock

A.J. Institute of Respiratory Medicine, Department of Medicine, University of Sydney, NSW, AUSTRALIA.

Current asthma, defined as the presence of both wheeze in the last 12 months and airway hyperresponsiveness (AHR), differentiates children with more severe abnormality from children with either measure alone. However the prognostic value of this epidemiological definition has not been well described. In 1982 we enrolled a random sample of 718 schoolchildren aged 8-10 years. We re-studied a representative sample of $573(80 \%)$ during 1997-1999, when the subjects were aged 22-27 years. AHR was defined as $20 \%$ fall in FEV1 at $<3.9 \mu \mathrm{mol}$ of histamine. Wheeze, sleep disturbance and activity limitation due to asthma during the last 12 months were measured by questionnaire. The outcomes in 1997-1999 were compared between groups classified by their status in 1982 .

\begin{tabular}{|l|c|c|c|c|}
\hline 1982 classification & $\begin{array}{c}\text { Normal } \\
n=483\end{array}$ & $\begin{array}{c}\text { Wheeze } \\
\text { only } \\
n=34\end{array}$ & $\begin{array}{c}\text { AHR only } \\
n=30\end{array}$ & $\begin{array}{c}\text { Current } \\
\text { asthma } \\
n=26\end{array}$ \\
\hline $\begin{array}{l}\text { Recent wheeze (\%) } \\
95 \% \mathrm{Cl}\end{array}$ & $\begin{array}{c}27.8 \\
(23.8,31.8)\end{array}$ & $\begin{array}{c}50.0 \\
(33.2,66.8)\end{array}$ & $\begin{array}{c}56.7 \\
(39.0,74.4)\end{array}$ & $\begin{array}{c}80.8 \\
(65.7,95.9)\end{array}$ \\
\hline $\begin{array}{l}\text { Sleep disturbance (\%) } \\
95 \% \mathrm{Cl}\end{array}$ & 9.5 & 20.6 & 13.3 & 46.2 \\
$(6.9,12.1)$ & $(7.0,34.2)$ & $(1.1,25.5)$ & $(27.0,65.4)$ \\
\hline $\begin{array}{l}\text { Activity limitation (\%) } \\
95 \% \mathrm{Cl}\end{array}$ & 4.8 & 8.8 & 20.0 & 34.6 \\
\hline $\begin{array}{l}\text { mean \%pred FEV } / \text { FVC } \\
95 \% \mathrm{Cl}\end{array}$ & $(2.9,6.7)$ & $(0,18.3)$ & $(5.7,34.3)$ & $(16.3,52.9)$ \\
\hline
\end{tabular}

There were statistically significant linear trends in all four outcomes measured $(P<0.001)$.

We conclude that this classification differentiates groups of children who have different prognoses. Furthermore, seventeen years after original classification, adults who were classified as having current asthma during childhood are much more likely to have 'asthma that matters'.

Support: CHATA, Allen + Hanburys, NHMRC, Asthma NSW, AstraZeneca
AT-HOME TREATMENT OF DEEP VENOUS THROMBOSIS (DVT) USING ENOXAPARIN IS MORE COST-EFFECTIVE THAN STANDARD CARE Josephine Weekley', Brian Smith', 2, Tim Howe3, Mark Hopps', Bob Bevan ${ }^{4}$ Dawn Brown ${ }^{4}$, Grace Leonello ${ }^{5}$ (1) Clinical Epidemiology and Health Outcomes Unit. (3) Registrar, (4) Interface Program and (5) Department of Finance. The Queen Elizabeth Hospital, SA 5011 and (2) Department of Medicine. The University of Adelaide. SA 5001

Introduction of low molecular weight heparins (LMWH) permit safe and effective treatment of DVT at home, for patients with minimal complications, compared to standard care involving hospital admission with continuous heparin infusion for 5 to 7 days 1.2. Substantial health-care costs savings have also been demonstrated ${ }^{3}$. The aim of this study was to conduct a cost analysis of DVT treatment at home with the LMWH enoxaparin, compared to standard inpatient care at The Queen Elizabeth Hospital (TQEH). Methods: Subjects presenting to the Emergency Department with principle diagnosis of DVT. or inpatients in whom DVT developed, were recruited over 1997-1999. Patients were eligible for inclusion according to criteria previously described ${ }^{2}$ and associated costs were tracked prospectively. Subjects were matched (2:1 where possible) to historical controls (1995/96) for age, gender and level of comorbidity (same or lower), checked with blinding by a physician uninvolved in the study. Control costs were obtained using the clinical costing system Trendstar ${ }^{(B)}$. Results: The mean total cost associated with at-home treatment was $\$ 649 \pm 15$ (SEM)( $n=28$ ) per patient, compared to $\$ 2,353 \pm 120$ (SEM) $(n=19)$ per patient receiving standard care, constituting a mean saving of $72.4 \%$ per patient. Minimal cost shifts of $<1 \%$ to patients and $7 \%$ to Medicare were demonstrated $(n=22)$. Recruitment was estimated as $29 \%$ of those eligible (62\% of total DVT coded patients) based on patient numbers for the financial year 1998/99. The gap between actual and potential recruitment was likely due to clinical practice preferences. Extrapolating from this recruitment data, estimated potential savings to the South Australian hospital systems range from $\$ 194,233$ to $\$ 415,257$ per year $(29 \%$ to $62 \%$ of eligible patients), based on the mean total number of DVT admissions to all metropolitan hospitals for the financial years 1995 to 1999. Conclusion: Athome treatment of uncomplicated DVT using enoxaparin is more cost-effective than standard care, without involving substantial cost-shifts.

1. Levine M et al, N Engl J Med 1996;334:677-81.

2. Koopman MMW et al, N Engl J Med 1996;334:682-87.

3. Spyropoulos AC et al, Arch Int Med 1999; 159(10):1139-40.

Key words: DVT, LMWH, cost-effectiveness

THE EFFECT OF COMBINED NICOTINE GUM AND NICOTINE PATCHES IN SMOKING CESSATION.

Richard Wood-Baker, Sylvia Cowles, Robert Ashwood \& Belinda Kellett. Department of Respiratory Medicine, Royal Hobart Hospital, Tasmania, 7000.

Smoking cessation is difficult to achieve and best outcomes are seen using nicotine replacement therapy. We have previously presented outcome data from the Smoking Cessation Program at our hospital'. Our data indicated that the 12 month abstinence rate was higher in smokers of less than 30 cigarettes/day $(20.4 \%$ ) than those who smoked 30 per day (16.4\%). Aim: to assess the effect of nicotine gum used in combination to a standard course of nicotine patches in heavy smokers. Methods: patients smoking

30 cigarettes/day presenting to the program were offered the opportunity to participate. In addition to standard interventions of counselling and nicotine patches, participants were provided with $4 \mathrm{mg}$ nicotine gum to use as required. At 3 months, all participants were contacted by telephone or letter and asked their current smoking status. Continued abstainers were asked to attend the clinic for measurement of breath carbon monoxide concentrations. Outcomes were compared with historical controls studied 18 months previously. Results: 107 participants were recruited over 4 months for the combination group. Their mean age was 45.0 (12.2 (SD)) years and $62.9 \%$ were female, similar to the control group of 116 whose mean age was 42.4 (11.8(SD)) years and $62.3 \%$ were female. Smoking uptake occurred at a similar age in the combination group and controls (15.5 (4.6(SD)) vs 16.0 $(5.8(S D))(p=0.48)$. However, there was a significant difference in the average number of cigarettes smoked per day $[41.7$ (12.6(SD)) vs 38.2 (11.1(SD)) $(p=0.36)]$ and the Fagerstrom score of nicotine addiction [8.2 (1.5(SD)) vs 7.5 $(1.9(S D))(p=0.0025)]$. At 3 months after entry into the program only $18.9 \%$ of the combination group were abstinent compared to $31 \%$ of historical controls. Breath carbon monoxide confirmed abstinence in the non-smokers, with a mean concentration of $2.5(2.2(\mathrm{SD})) \mathrm{ppm}$. Conclusion: The addition of nicotine gum to topical replacement did not improve 3 month abstinence rates in heavy smokers. This may reflect greater nicotine addiction in the experimental group when compared to historical controls.

1 The prevalence of cigarette smoking and smoking cessation rates in a hospital population. Richard Wood-Baker. TSANZ Annual Scientific Meeting, Adelaide, 1998 
SURVEILLANCE OF AUSTRALIAN WORKPLACE BASED RESPIRATORY EVENTS (THE SABRE PROJECT)

Michael Abramson, David Elder, Malcolm Sim

Department of Epidemiology \& Preventive Medicine. Monash Medical

Introduction: The Surveillance of Work related Occupational Respiratory Disease (SWORD) program has recently published its ten year results providing incidence rates and causative agents for occupational lung diseases in the UK. In Australia the only comparable scheme to report on a similar range of conditions is the SABRE scheme.

Methods: A notification form is mailed regularly to 46 full members of the TSANZ and 26 fellows of the Australasian Faculty of Occupational Medicine resident in Victoria and Tasmania.

Results: A total of 1382 forms including nil returns were returned over the first two years of the scheme. The mean (SD) age of the 337 patients notified was $53.5(15.9)$ years. There were $287(85 \%)$ males and 45 females. There injuries, 30 pneumoconiosis, 33 mesothelioma, 85 nonmalignant pleural disease ( 71 predominantly plaques and 14 diffuse thickening), 5 occupational lung cancers, 4 infectious diseases and 21 with another diagnosis. The most common agent reported was asbestos in 123 cases. The most common agent reported in asthma was wood dust in 21 cases.

Conclusions: Occupational asthma and nonmalignant pleural disease are the most commonly reported conditions in a voluntary reporting scheme involving respiratory and occupational physicians. The pattern of occupational lung diseases in Australia is similar to that of overseas schemes. Further studies are being undertaken to validate these diagnoses.

Supported by the Australian Lung Foundation/Dust Diseases Board

Key words: Occupational lung disease, epidemiology School. The Alfred Hospital, Prahran 3181, Australia were 5 cases of allergic alveolitis, 120 asthma, 30 bronchitis, 26 inhalation

Nominations for Awards: $\mathrm{Ni}$

THE SURVIVAL OF PATIENTS WITH CHRONIC AIRFLOW LIMITATION ON HOME OXYGEN THERAPY OVER A 22 YEAR PERIOD

Alan Crockett', Josephine Cranston', John Moss², John Alpers'1.

1. Respiratory Unit, Department of Medicine, Flinders Medical Centre, Bedforo Park, South Australia, 5042

2. Department of Public Health, University of Adelaide, South Australia. 5005.

The aim of this study was to determine whether the surviyal of patients with Chronic Airflow Limitation (CAL) on home oxygen therapy (HOT) seen in routine clinical practice was less than that of the original Randomized Controlled Trials (RCTs) and to investigate factors influencing survival. Methods: A list of recipients prescribed HOT for CAL at Flinders Medical Centre from 1 January 1977 to 31 March 1999 was generated retrospectively. Survival data were compared with that reported for the two original RCTs and recent overseas studies. Factors influencing survival were studied. Results: Survival data were collected for $505 \mathrm{CAL}$ patients on HOT (male:female 249:256) mean age (males, females) 69.9, 71.0 years. Survival was less than that of the original RCTs but comparable to that reported for Belgian and Swedish CAL patients. Overall crude survival was $75 \%$ at 1 year, $51 \%$ at 2 years, $19 \%$ at 5 years and $1 \%$ at 10 years. Median survival was 21.9 months for males and 28.2 months for females with a significant survival advantage for female sex (log rank test $p=0.016$ ). Cox's Proportional Hazard modelling showed a lower body mass index (BMI) was significantly associated with reduced survival for males while for females age, co-morbidity, BMI, and FEV, at baseline were related to survival. CAL patients using more than 19 hours per day on their oxygen concentrator had improved survival over those using less than 19 hours per day. Conclusions: Survival for CAL patients on HOT, where the tighter selection criteria of the original RCTs do not always apply, is less than that of the original RCTs but comparable to that recently reported overseas. Gender differences are apparent.

Supported by: NHMRC Evidence Based Clinical Practice Research Program Key words: Chronic Airflow Limitation, home oxygen therapy, survival

\section{CLINICAL MEASUREMENT OF HUMAN TRACHEAL MUCUS VELOCITY} (TMV)

Lucy Morgan', Mark Pearson'2, Douglas Mackey², Hans Van der Wall2, Robbert de longh ${ }^{1}$, Matthew Peters ${ }^{1}$, Jonathan Rutland Departments of Respiratory Medicine(1), and Nuclear Medicine(2), Concord Repatriation General Hospital. NSW 2139

Introduction: Mucociliary clearance (MCC) removes inhaled particulate matter, microorganisms, excess secretions and cellular debris from the respiratory tract. MCC is impaired in many respiratory conditions including chronic aiflow limitation (CAL) and may vary over time, during acute exacerbations and with pharmacological intervention. Current methods of measuring MCC are limited by long acquisition time, radiation dose, difficulty of aerosol generation and deposition, signal attenuation and the need for patient cooperation. Aims: (i) To develop a rapid, simple, direct measurement of TMV for assessment of MCC suitable for clinical and research use; (ii) To compare TMV in normal subjects with that of CAL patients. Methods: A $0.1 \mathrm{ml}$ droplet of macroaggregated albumin labelled with $99 \mathrm{mTC}(2-5 \mathrm{MBq})$ was injected, atter cutaneous application of local anaesthetic, through the cricothyroid membrane into the trachea of a seated subject. For data acquisition the subject lay supine for 15 min under a GE 400 gamma camera interfaced to an Icon workstation (Siemens). All data were stored in Icon Interfile format and analysed by CiliaCO, a program written in IDL specifically for the project. TMV was measured in 14 normal subjects and 17 patients with CAL. Results: The test was well tolerated and no adverse events were reported. TMV (mean \pm SEM) in normal subjects was $8.0 \pm 0.8 \mathrm{~mm} / \mathrm{min}$. TMV (mean \pm SEM) for patients with CAL was $0.6 \pm 0.8$ $\mathrm{mm} / \mathrm{min}$. TMV was significantly different in CAL patients than normal subjects $(p<0.001$, Student's $t$-test). Conclusions: We have developed a rapid, safe, direct measure of TMV that gives values similar to those reported elsewhere. Using this technique we have shown that TMV is significantly slower in patients with CAL than in normal subjects.

Supported by the ALF/ Boehringer Ingelheim CAL Fellowship Key words: mucociliary clearance, scintigraphic, CAL
MORBIDITY AND 3 MONTH MORTALITY POST LUNG VOLUME REDUCTION SURGERY (LVRS): A MULTI-CENTRE ANALYSIS. FA Finlayson, S Reid, C Franklin, M Bailey, EH Walters, JA Smith, GI Snell. The Alfred Hospital and Monash University, Melbourne, Victoria. The Australia and New Zealand (ANZ) LVRS Database was established in 1997 to provide clinicians in Australia with information that leads to the best possible outcomes for people who undergo LVRS. At October 1999, the database contained individual patient data from 235 cases from 10 centres. The data have been entered and verified in Access 97 and analysed in SPSS version 9 . Univariate and multivariate analysis was undertaken to examine the effect of pre-operative medical and peri-operative surgical factors on morbidity and 3 month mortality post surgery. $129(55 \%)$ cases were male.

Mean age at surgery was 62.1 (SD 7.7) years [ $n=230]$. Pre-operatively, mean FEV $1=0.77 \mathrm{~L}$ (SD 0.25$)[\mathrm{n}=228]$ a and mean $6 \mathrm{MW}$ $=323.5 \mathrm{~m}$ (SD 113.5) [n=202].

MULTIVARIATE ANALYSIS

\begin{tabular}{|l|l|l|l|}
\hline OUTCOME & $\begin{array}{l}\text { Variable with greatest effect } \\
\text { size (\%)/ odds ratio [OR] }\end{array}$ & $\begin{array}{l}\text { Regression } \\
\text { co-efficient }\end{array}$ & p value \\
\hline \multirow{4}{*}{ Length of stay } & No complications (19.7) & -0.75 & 0.0001 \\
\cline { 2 - 4 } & Pre-op 6MW (7.5) & -0.001 & 0.0001 \\
\cline { 2 - 4 } & Thoracotomy (2.1) & -0.37 & 0.019 \\
\hline \multirow{2}{*}{$\begin{array}{l}\text { Intercostal drain } \\
\text { time }\end{array}$} & No complications (20.6) & -0.74 & 0.0001 \\
\cline { 2 - 4 } & Apical resection (5.9) & 0.52 & 0.0002 \\
\cline { 2 - 4 } & Pre-op 6MW (4.2) & -0.001 & 0.0011 \\
\hline \multirow{3}{*}{$\begin{array}{l}\text { Death within 3 } \\
\text { months }\end{array}$} & $\begin{array}{l}\text { Thoracotomy } \\
\text { [15.6 Cl 2.8-87.4] }\end{array}$ & 2.70 & 0.02 \\
\cline { 2 - 4 } & $\begin{array}{l}\text { ICC time (log e) } \\
\text { [ 3.86 Cl 1.65-9] }\end{array}$ & 1.39 & 0.02 \\
\hline
\end{tabular}

Post-operative physiological (FEV1) and functional ( 6 min walk) parameters improve to 12 months. Pre-operative functional status and surgical factors have the greatest effect on morbidity and 3 month mortality post-operatively.

Acknowledgements:

Boehringer Ingelheim, John Clarke-Advanced Biomedical, Victorian

Tuberculosis and Lung Association, Contributing Centres 
CYTOKINE GENE POLYMORPHISMS IN COPD

Yang $\mid A^{1}{ }^{1}$, Rabnott GC1, Tunnicliffe AM11, Dent AG1, Slaughter R1, Zimmerman PV', Fong KM ${ }^{1}$

1 Division of Thoracic Medicine. The Prince Charles Hospital;

2Department of Medicine, University of Queensland, Brisbane QLD 4032

Chronic obstructive pulmonary disease (COPD) is characterised by abnormal airways inflammation in response to inhaled toxins. Functionally relevant polymorphisms of key cytokines that mediate inflammation have recently been characterised. Myeloperoxidase (MPO) is a neutrophil lysosomal enzyme that produces bactericidal oxidants and bioactivates toxins in tobacco smoke. Polymorphism at position -463 of the MPO promoter removes a transcription factor binding site thereby reducing MPO expression. Variable numbers of tandem repeats in intron 2 of the interleukin-1 receptor antagonist (IL-1Ra), an anti-inflammatory cytokine, alter IL-1Ra production. A base pair substitution (TNF2 allele) at position -308 of the tumour necrosis factor-alpha (TNF- $\alpha$ ) promoter enhances TNF- $\alpha$ expression in vitro. Airway TNF- $\alpha$ and MPO levels increased are COPD, and IL-1Ra expression is increased in asthmatic airways. Aim: To examine MPO, IL-1Ra and TNF- $\alpha$ polymorphisms as susceptibility and disease-modifying genes in COPD. Method: Genotypes were determined by PCR in 133 Australian adults with COPD and 128 anonymous blood donors. Results: COPD patients (65\% male) had mean (SD) age 69.6 (8.5) yr. FEV $48.2(19.7) \%$ pred and smoking 54 (33) pack-yr. Variant allele frequencies in COPD were MPO-A 22\%; TNF2 23\%; IL11Ra*2 29\%, IL-1Ra*3 3\%, which did not differ from controls. Variant alleles for IL-1Ra tended to be more frequent in COPD (32.2\%) compared with controls $(23.6 \%)(p=0.07)$. IL-1Ra*2 patients had lower mean KCO $(55.8 \%)$ than those without IL-1Ra*2 $(64.4 \%)(p<0.05)$. TNF- $\alpha$ and MPO genotypes did not predict FEV $1, K C O$ or chronic bronchitis. Conclusions: IL-1Ra may influence disease severity and susceptibility in COPD. Our present data do not provide evidence for MPO or TNF- $\alpha$ as susceptibility genes or modifiers of lung function in COPD. Recruitment is ongoing, to achieve sufficient power to exclude effects in COPD.

Supported by: The Prince Charles Hospital Foundation, NHMRC Postgraduate Medical Scholarship

Key words: COPD, cytokines, polymorphisms

Nomination for Prizes:

EFFECT OF AN EVIDENCE-BASED GUIDELINE FOR CHRONIC OBSTRUCTIVE PULMONARY DISEASE (COPD) ON PATIENT OUTCOMES.

Frida Cheok', Kieran McCaul1, Brian Smith22, Richard Ruffin'3, Anne Marie Southcott4, Peter Frith ${ }^{5}$, Ral Antic 6 .

(1) Epidemiology Branch, Dept Human Services, SA 5000. (2) Clinical Epidemiology Unit. The Queen Elizabeth Hospital (TQEH), SA 5011, (3) Dept of Medicine, TQEH, (4) Respiratory Medicine. TQEH, (5) Respiratory Department. Flinders Medical Centre, SA 5042, (6) Thoracic Medicine, Royal Adelaide Hospital, SA 5000 .

Introduction: Despite the proliferation of clinical practice guidelines, there is limited evidence of their uptake in clinical settings, and even less evidence of impact on patient health outcomes. The Adelaide Collaboration on Chronic Obstructive Respiratory Disease (ACCORD) conducted a trial to evaluate the effect of a management guideline, developed from the best available evidence, on process, impact and patient outcomes. Methods: Between May 1998 and June 1999, 1250 subjects with COPD were enrolled from 4 hospitals, using a pre-post study design, with two metropolitan hospitals assigned to the control arm and two to the intervention arm. Clinical and process measures were monitored for all subjects and health status data collected from consenting subjects at baseline (in hospital), 6 weeks and 6 months (Seattle Obstructive Lung Questionnaire (SOLQ) and General Health Questionnaire, 28 item version (GHQ-28)). Impact of the intervention on length of stay, re-admission rates, and patient health status were assessed on an "intention to treat" basis initially, then adjusted for actual use of the guideline. Results: On the basis of intention to treat, no evidence was found for an effect of the intervention on patient quality of life or mental health status. After adjustment for age and sex, there were indications of a reduction in risk of re-admission associated with the intervention, but this was not statistically significant. Considerable variation in the actual use of the guideline was found, however, and further analyses will be conducted to adjust for this. Conclusions: Our findings support the view that there is a need for research on the implementation of evidencebased medicine and for outcomes to be linked to guideline use.

Key words: Guidelines, COPD, outcomes, uptake
CARDIORESPIRATORY RESPONSES TO INCREMENTAL SHUTTLE WALKING TEST (ISWT) AND SIX MINUTE WALKING TEST (6MWT) IN CHRONIC OBSTRUCTIVE PULMONARY DISEASE (COPD) JENKINS SC 1,3, CECINS NM ${ }^{1}$, ALLEN-WILLIAMS $\mathrm{S}^{3}$, EASTWOOD PR \& HILLMAN DR ${ }^{2}$

(1) Departments of Physiotherapy and (2) Pulmonary Physiology. Sir Charles Gairdner Hospital. Perth 6009. (3) School of Physiotherapy, Curtin University of Technology. Perth. Western Australia 6008

The 6MWT and ISWT are commonly used measures of exercise capacity in patients referred for pulmonary rehabilitation. Previous research has shown lower heart rate (HR) and dyspnoea levels with the $6 \mathrm{MWT}$, indicating that it may only submaximally stress the cardiorespiratory system. Aim: To compare the cardiorespiratory responses associated with the $6 \mathrm{MWT}$ and ISWT in patients with COPD. Methods: 15 patients (12 male), mean (SD) age $62.6(7.7)$ years and FEV $, 0.91(0.47) 1,31.4$ (13) \%predicted were studied. ISWT and 6MWT were conducted using standard protocols with strong encouragement to maximise performance. HR (Polar monitor) and dyspnoea (Borg 0-10 scale) were measured at one minute intervals during both tests. Oxygen saturation $\left(\mathrm{SpO}_{2}\right)$ was measured pre- and immediately post-exercise. Results: There were no significant differences in maximum $\mathrm{HR}_{1}$ dyspnoea or the magnitude of desaturation with the two tests (Table). Mean(SD) distance walked in the ISWT and 6MWT was 397(136) and $507(108) \mathrm{m}$ respectively.

\begin{tabular}{|c|c|c|c|c|c|c|}
\hline & \multicolumn{2}{|c|}{ HR (bpm) } & \multicolumn{2}{|c|}{$\mathrm{SpO}_{2}(\%)$} & \multicolumn{2}{|c|}{ Dyspnoea } \\
\hline & Pre-ex. & Max-ex. & Pre-ex. & Post-ex. & Pre-ex. & Max-ex. \\
\hline ISWT & $89.6(12)$ & $125.1(18)$ & $95.5(1.5)$ & $86.3(6.3)$ & $0.6(0.8)$ & $6.0(2.2)$ \\
\hline 6MWT & $91.9(13)$ & $126.9(15)$ & $94.9(1.6)$ & $86.8(5.6)$ & $0.6(0.8)$ & $5.9(2.1)$ \\
\hline
\end{tabular}

Conclusions: With strong encouragement the $6 \mathrm{MWT}$ and ISWT can provide equivalent degrees of cardiorespiratory stress. However, unlike the $6 M W T_{t}$ the external pacing and standard increments in workload of the ISWT enables comparison between patients at equivalent workloads and the prescription of a walking program based on workload.

1Singh SJ et al (1992): Thorax 47: 1019-1024.

Key words: ISWT, 6MWT, chronic obstructive lung disease

REDUCED CONTRIBUTION OF THE DIAPHRAGM TO POSTURAL CONTROL IN PATIENTS WITH SEVERE CHRONIC AIRFLOW LIMITATION

P.W. Hodges, D.K. McKenzie, I. Heijnen and S.C. Gandevia

Prince of Wales Medical Research Institute. Sydney, NSW 2031

Contraction of the human diaphragm contributes to the response of the trunk muscles that stabilises the spine during rapid limb movements. This postural function must be coordinated with the respiratory activity of the diaphragm. During repetitive limb movement there is usually phasic modulation of diaphragm electromyographic activity (EMG) with respiration, as well as tonic activity and phasic modulation with each limb movement. Aim: To determine whether this pattern of activity occurs in subjects with increased respiratory demand. Methods: Two groups were tested: (i) six subjects with severe chronic airflow limitation (CAL) who were awaiting lung volume reduction surgery; and (ii) nine subjects with no history of respiratory disease who breathed with an increased dead space for 4 minutes. EMG recordings of the right costal diaphragm were made with intra-muscular electrodes inserted into the $7^{\text {th }}$ or $8^{\text {th }}$ intercostal space. Movement of the left arm was recorded with a potentiometer. Patients with CAL performed rapid repetitive shoulder movements $\left(30^{\circ}\right)$ for $10-20 \mathrm{~s}$ while standing or sitting. The normal subjects moved their left arm for the first $10 \mathrm{~s}$ of each minute of dead-space breathing. The contribution of the diaphragm to respiration and postural control was assessed by evaluation of the frequencies of phasic modulation of EMG amplitude. This was quantified by analysis of the distribution of power in the power spectral densities. Results: Unlike normal subjects, the CAL subjects had no or minimal modulation of diaphragm EMG at the frequency of limb movement. In the trials in which normal subjects breathed with increased dead space the power of the EMG data at the movement frequency was significantly reduced by the second minute. Conclusions: These results indicate that in situations of increased respiratory demand the contribution of the diaphragm to postural control is reduced.

Supported by the NHMRC and the Australian Lung Foundation. 


\section{SOCS-1: A KEY REGULATOR OF INTERFERON-GAMMA SIGNALLING} IN VIVO

W.S.Alexander, R.Starr, S.Nicholson, D.Metcalf, A.Elefanty, M.Brysha, T.Willson, E.Viney, N.Sprigg, A.Farley, S.Rakar, J.Corbin, S.Mifsud, L.DiRago, N.Nicola, and D.Hilton. The Walter and Eliza Hall Institute of Medical Research and The Cooperative Research Centre for Cellular Growth Factors. Post Office. Royal Melbourne Hospital, Vic. 3050

SOCS (suppressor of cytokine signalling)- 1 is one of a family of eight proteins containing an SH2 domain and C-terminal SOCS Box motif. To explore the physiological roles of the SOCS proteins, we are generating mice in which these genes are replaced by B-galactosidase. SOCS-1-/- mice were born but exhibited stunted growth and died before weaning with fatty degeneration of the liver and monocytic infiltration of several organs. B-galactosidase activity in SOCS-1 +1 - mice was detected in most cells within the thymus, as well as in splenocytes and bone marrow cells, particularly in B-lymphoid populations. Consistent with this expression profile, the thymus in diseased SOCS-1-/- mice was significantly reduced in size, the animals were lymphopenic and their haemopoietic organs exhibited deficiencies in B-lymphocytes. These data suggest that mice lacking SOCS-1 are unable to properly control responses to cytokines that have cytotoxic side-effects. As neonatal exposure to interferongamma is known to cause liver damage and lymphopenia, we investigated the role of this cytokine in the pathology of Socs-1-deficient mice. Macrophages derived from SOCS-1-deficient mice were markedly hypersensitive to the effects of interferon-gamma in assays of parasite killing, and the mice themselves were resistant to normally lethal viral challenge. Remarkably, damage to multiple organs and neonatal death of SOCS-1-/-mice was completely overcome by treatment with neutralising antibodies to interferongamma or by the production of mice lacking interferon-gamma in addition to SOCS-1. Thus SOCS-1 is a key physiological regulator of interferon-gamma signalling that is critical to balance the beneficial effects of this cytokine with potentially devastating side-effects.

\section{REMODELLED BRONCHIAL VESSELS IN CHRONIC BRONCHITIS AND} CYSTIC FIBROSIS

JW Wilson, X Li, S Wilson. $\mathrm{N}$ Harley, V Olsen, ATC.Kotsimbos and J Rolland.

Dept. of Respiratory Medicine, The Alfred Hospital, Prahran, Vic, Australia; University Medicine, Southampton General Hospital, Southampton, Hants, United Kingdom and Dept. of Pathology and Immunology, Monash University, Prahran, Vic, Australia.

Severe chronic bronchitis (CB) and cystic fibrosis (CF) are characterized by fixed aiflow obstruction and respiratory failure. Remodelling of the airways may involve bronchial vessels, as has been shown in asthma (Li \& Wilson AJRCCM 1997;156:229). We hypothesize that remodelled airways in CB and CF are associated with angiogenesis and increased vascularity. Using airways taken from subjects at the time of lung transplantation (CB, $n=10$ and $C F, n=10$ ) or recent post mortem controls $(C, n=10)$, we measured the number and area coverage of sections with blood vessels. Detection was achieved with monoclonal antibodies to Factor VIII Ag, collagen type IV, non-lymphatic vessels (PAL-E) and CD31. CF airways had more vessels than controls and CB using coll IV (504 vs $330 / \mathrm{mmsq} p<0.01$ and 354/mmsq $p<0.05$ respectively).

Non-lymphatic vessels accounted for a significant component of the increase, and was higher in $\mathrm{CF}(\mathrm{C}=205, \mathrm{CB}=223, \mathrm{CF}=369 / \mathrm{mmsq}, \mathrm{p}<0.05)$. There was no difference between groups for Factor VIII Ag positive vessels ( $\mathrm{C}=187, \mathrm{CB}=171, \mathrm{CF}=227 / \mathrm{mmsq}$ ). Numbers of vessels detected were greater for coll IV than for Factor VIII in all specimens $(p<0.001)$. Severe $C F_{1}$ but not $\mathrm{CB}$ is characterized by increased vessel numbers and is due in part to non-lymphatic vessel proliferation. We thank the Alfred Heart-Lung Transplant Service for their co-operation.

Supported by NHMRC and The Alfred Whole Time Medical Specialists Scheme.
IMMUNOHISTOCHEMICAL ANALYSIS OF PROTEASE ACTIVATED RECEPTOR (PAR) EXPRESSION IN HUMAN ASTHMATIC AIRWAYS. Darryl Knight ${ }^{1,2}$, Sam Lim ${ }^{3}$, Amelia Scaffidi 1,2, Nicholas Roche ${ }^{3}$, K.Fan Chung ${ }^{3}$, Geoffrey Stewart ${ }^{4}$ \& Philip Thompson ${ }^{1,2}$.

\section{(1) Asthma \& Allergy Research Institute. (2) Department of Medicine.} University of Western Australia. (3) National Heart \& Lung Institute. (4) Department of Microbiology, University of Western Australia. PAR are a family of G-protein coupled receptors, auto-activated via proteolytic cleavage of the extracellular amino terminus. PAR-1,-3 and -4 are activated by thrombin, whereas PAR-2 and -4 are activated by trypsin (tryptase). PAR-2 activation exerts a protective effect in mouse airways, suggesting an important role in the airways response to inflammation. We hypothesized that PAR-1 and PAR-2 expression would be altered in asthma. Methods: Bronchial biopsies were obtained from 10 normal controls and 20 asthmatics, including 10 who were using inhaled steroids. Frozen sections were stained with monoclonal antibodies to either PAR-1 or PAR-2, visualized with $\mathrm{DAB}$ and scored semi-quantitatively for staining intensity and cellular distribution. Results: Specific PAR staining was seen in epithelium (epi), airway smooth muscle (ASM) and macrophages. In normal subjects, epi PAR-1 staining was significantly greater than for PAR-2 and was confined to the apical region of columnar cells. PAR-2 staining was homogeneous throughout the epi. The intensity of PAR-1 staining in asthmatic biopsies was not different to contro|s, although the cellular distribution was distinctly different, appearing diffuse and widespread. In contrast, the intensity of epi PAR-2 staining was significantly increased over control. In biopsies from asthmatics taking inhaled steroids, epi staining for PAR-1 and PAR-2 was similar to controls. Expression of PAR-1 and PAR-2 in ASM was not different between the groups. Conclusion: These findings suggest that asthma per se influences the expression of both PAR-1 and PAR-2 in human bronchial epithelium. The expression of PAR-2 appears to be inducible and may initiate protective mechanisms in response to airway inflammation.

Supported by the NHMRC and Asthma Foundation of W.A.

Key words: Protease Activated Receptor, epithelium, inflammation, asthma.

\section{EXPRESSION OF METALLOPROTEINASES IN BRONCHOALVEOLAR LAVAGE FLUID OF LUNG TRANSPLANT RECIPIENTS}

M Tamm, M Roth, H Parsons, M Malouf, P Chhajed, P Johnson, J Black. A Glanville

Heart Lung Transplant Unit, St Vincent's Hospital and Department of Pharmacology, University of Sydney, Sydney, Australia

The composition of the extracellular matrix (ECM) plays an important role in aiway remodelling and increased ECM deposition is causally involved in fibrotic processes, The composition of the ECM is regulated by the balance of de novo synthesis of ECM, degradation of ECM by matrix metalloproteinases (MMP) and by the activity of their respective inhibitors. We investigated the expression of MMPs in bronchoalveolar lavage fluid (BAL) of patients following lung transplantation and compared the results to histopathological and microbiological findings. MMPs were determined by Zymography as previously described (AJRCCM 1997; 156: 1987). BAL samples of patients with acute rejection $(n=15)$ were compared to those obtained from patients with bacterial or fungal infection $(n=19)$ and to controls $(n=17)$. In addition, MMPs of patients treated for rejection were analysed. MMP-2 and MMP-9 were expressed in all BAL samples. Patients with acute rejection showed a marked increased expression of MMP-2 and a moderately elevated expression of MMP-9 compared to controls. The active form of MMP-2 was typically expressed in BAL samples of patients with bronchial rejection. The amount of MMP-2 and MMP-9 decreased after successful treatment for rejection. The degree of MMP-2 and MMP-9 expression varied in BALs of patients with infection.

Conclusion: the expression of MMP-2 might be a useful marker for airway remodeling and treatment success in lung rejection. Further studies will analyse the balance of MMPs and their respective inhibitors. 
A KEY ROLE FOR B CELLS IN ASTHMA: BRIDGING THE GAP BETWEEN ALLERGEN RECOGNITION AND TISSUE DAMAGE. Alusha A Mamchak Lung Disease Research Group. Department of Pharmacology, University of Melbourne Victoria 3010.

An initial event in the development of asthma is the recognition of allergens by cells of the immune system. Allergens may be recognised by $B$ cells and dendritic cells (DC), which process the allergen into peptides. Processed peptides are subsequently expressed on the cell surface in association with the major histocompatability complex class II (MHC-II). DC migrate to the draining lymph nodes where they present the peptide/MHC-Il complex to T cells, resulting in the activation of antigen-specific $T$ cells. Following activation, antigen-specific T cells can provide "help" to $B$ cells presenting an appropriate peptide/MHC-II combination. T cell "help" is provided by the expression of a new cell surface molecule, CD4OL, and the secretion cytokines. It is through the provision of T cell "help" that B cells become activated and differentiate into immunoglobulin ( $\mathrm{lg})$ secreting and memory cells. Igs have a number of isotypes each of which mediate different effecto functions. The $\mathrm{lg}$ isotype that is produced as a result of successful T / B collaboration is determined by the pattern of $T$ cell derived cytokines. In asthma, the formation of IgE secreting cells driven by the cytokines, interleukin (IL-)4 and IL-13, is detrimental. $\operatorname{lgE}$ binds to a receptor (FcERI/II) expressed on the surface of mast cells. When $\lg E$ binds to an allergen resulting in the cross-linking FceRI/II, it induces a signal that results in mast cell degranulation, releasing inflammatory mediators that cause tissue damage and ultimately lead to airway remodeling. Thus, the B cell by secreting $\mathrm{lgE}$ forms a bridge between the initial recognition of an allergen and ensuing tissue damage. While the recognition of allergens and subsequent production of Ig does not normally lead to disease, in asthmatics the immune response appears to be inappropriately regulated thereby allowing hyperactivation of the immune system. Asthma has the hallmarks of an inheritable trait, and one possibility is that mutations that increase the sensitivity of $B$ cells to, or enhance the production of, IL-4 or IL13 may lead to a predisposition to asthma.

BRONCHOALVEOLAR LAVAGE (BAL) MACROPHAGE AND LYMPHOCYTE PHENOTYPES IN LUNG TRANSPLANT RECIPIENTS. Ward $C$, Whitford $H$, Snell GI, Bao $H$, Zheng $L$, Kotsimbos ATC, Williams TJ and Walters EH.

Respiratory Medicine. Alfred Hospital and Monash University Melbourne. There is little information regarding the role of the alveolar macrophage (AM) in lung transplantation. We hypothesised that changes in BAL AM and lymphocyte phenotype would be apparent even in stable lung transplant recipients (SLTR) and may be important in understanding the pathophysiology of Bronchiolitis Obliterans Syndrome (BOS). We performed a cross sectional study, using a standardised $3 \times 60 \mathrm{~mL}$ BAL and flow cytometry in 19 SLTR, 5 subjects with BOS and 18 normals. We found significantly elevated neutrophils in the SLTR (median 4.5\%, range 2.5-30.7 versus normal; median $1 \%$, range $1-5 ; \mathrm{P}<0.05)$, with a frank elevation in the BOS subjects (median $12.6 \%$, range $2.5-30.7 ; P<0.05$ compared to normals: $\mathrm{P}=0.08 \mathrm{BOS}$ versus stable). Our lymphocyte data showed increased numbers of: Natural Killer (CD56/CD16 positive) cells, CD11b and CD11c on CD3 cells, CD8 positive lymphocytes and increased HLADR expression on CD8 cells in SLTR and BOS versus normals. In contrast, expression of surface markers associated with a range of AM host defence functions against bacteria, fungi and viruses, were significantly lower in lung transplant recipients: AM data expressed as mean channel fluorescence. ${ }^{*} P<0.05,{ }^{*} P<0.005$ versus normals.

\begin{tabular}{|l|c|c|c|c|c|}
\hline & CD14 & HLADR & CD11a & CD11b & CD11c \\
\hline Normals & 1.9 & 29 & 10.9 & 5.7 & 21.9 \\
& $1.4-2.9$ & $21-59$ & $6-20$ & $2.8-11$ & $11-38$ \\
\hline SLTR & $1.3^{*}$ & $13^{*}$ & $5^{* *}$ & $2.2^{*}$ & $10.7^{* *}$ \\
& $1-3.9$ & $3-54$ & $1.5-14.6$ & $0.9-10$ & $1-26$ \\
\hline BOS & $1.7 n . s$ & $23^{*}$ & $4^{*}$ & $5.2 n . s$ & $7.5^{*}$ \\
& $1.1-2.0$ & $12-41$ & $1.7-16.8$ & $1.8-7.5$ & $5.6-22$ \\
\hline
\end{tabular}

Our novel findings may be consistent with a situation in which stable allografts undergo complex lymphocyte and macrophage changes that may result from clinically silent processes of infection, partially suppressed rejection, or both.

Supported by Glaxo-Wellcome, Australia.

Key words: Transplantation, BAL, Macrophage, Infection, Rejection.
HLA-DR EXPRESSION ON TISSUE RESIDENT CELLS IN ASTHMA AND CHRONIC OBSTRUCTIVE PULMONARY DISEASE.

N Carroll1, S Mutavdzic'1, J Elliot'1, K Mackay², A James'1.

(1) Dept Pulmonary Physiology, Sir Charles Gairdner Hospital, Perth 6009. (2) Children's Chest Research Centre. The New Children's Hospital, Sydney 2124. Australia.

Asthma and COPD are characterised by persistent airway inflammation. We hypothesised that this results from persistent immune stimulation by activated tissue-resident cells in situ in smokers who develop COPD and in asthmatics. We measured HLA-OR expression on fibroblasts, resident macrophages (RM), lymphoid aggregates (LA), epithelium (Epi), smooth muscie (ASM) and mucous glands (G) in cases of COPD and asthma of varying severity. Transverse sections of 3 or 4 large airways from controls (CO), smokers with normal lung function (SM), mild (FEV1<80\%) and severe (FEV $1<20 \%$ ) COPD and mild (nonfatal - NFA) and severe (fatal FA) asthma; $n=8$ in each group, were stained with HAM56 and anti-HLA-DR monoclonal antibodies. HLA-DR+ celis counted in the submucosa were expressed as number (mean \pm SE) per $\mathrm{mm}$ of the BM while LA, Epi, ASM and $\mathrm{G}$ were scored semiquantitatively $(0-100 \%)$.

$\begin{array}{lllllll} & \text { CO } & \text { SM } & \text { NFA } & \text { FA } & \text { mCOPD } & \text { sCOPD } \\ \text { Fibros HLADR+ } & 0.01 \pm 0.0 & 0.01 \pm 0.0 & 0.01 \pm 0.01 & 0.03 \pm 0.01 \cdot & 0 \pm 0 & 0 \pm 0 \\ \text { RM's HLADR+ } & 0.03 \pm 0.01 & 0.03 \pm 0.01 & 0.02 \pm 0.01 & 0.1 \pm 0.03^{\circ} & 0 \pm 0 & 0.01 \pm 0.01\end{array}$
Cases of COPD had the highest expression of HLA-DR on epithelium and glands while CO and SM had the highest on ASM. The NFA group had the lowest HLA-DR expression on all resident cells. Cases of COPD and FA had a high \% of airways with LA's but HLA-DR expression was only seen in LA's in FA cases. HLA-DR staining on fibros and TRMs was very low and only increased in cases of FA, possibly due to an acute inflammatory stimulus. The findings suggest that activation of structural cells is widespread in all cases and may contribute to persistent airway inflammation.

Support: NHMRC Australia.

Key words: Asthma, COPD, inflammation, HLA-DR Awards: Nil

HUMAN EOSINOPHIL-AIRWAY SMOOTH MUSCLE CELL INTERACTIONS INVOLVE VCAM-1 AND ICAM-1

J Margaret Hughes ${ }^{1}$, Steve M Carlin², Peter RA Johnson², Carol L Armour1 Respiratory Research Group. Departments of Pharmacy ${ }^{1}$ and Pharmacology2,University of Sydney, NSW 2006

The airways of asthmatic subjects typically contain eosinophils, have increased amounts of ainway smooth muscle and are hyperresponsive. Eosinophils release a variety of mediators implicated in the signs and symptoms of asthma. We have previously established that in co-culture eosinophils attach rapidly to human airway smooth muscle cells (ASMC), with maximal adhesion occurring after $2 \mathrm{~h}$ and then falling gradually to $50 \%$ of maximum by $20 \mathrm{~h}$. The aim of this study was to determine whether the cellular adhesion molecules VCAM-1 and ICAM-1 are involved in human eosinophilairway smooth muscle cell interactions. Methods: ASMC were plated into slide wells at $2.5 \times 10^{5}$ celis/well and cultured for $48 \mathrm{~h}$. Purified eosinophils were obtained from the blood of healthy volunteers using immunomagnetic cell sorting and $2 \times 10^{5}$ cells/well added to the ASMC for 2 or $20 \mathrm{~h}$ in the presence or absence of antibodies to VCAM-1 and ICAM-1 at 1,3 and $10 \mu \mathrm{g} / \mathrm{ml}$. Culture medium and non-adherent eosinophils were removed and the remaining attached cells washed 3 times, fixed and stained with Kimura Light. Adherent eosinophils were identified and counted across 20 fields of view using light microscopy and 200x magnification. Results: Eosinophil adhesion to airway smooth muscle cells treated with anti-VCAM- $1(3 \mu \mathrm{g} / \mathrm{ml})$ was significantly $(p<0.05, n=4)$ reduced to $71.7 \pm 4.2 \%$ of control after $2 h$, but not after $20 \mathrm{~h}$, of co-culture. In contrast, adhesion in the presence of anti-ICAM- $1(3 \mu \mathrm{g} / \mathrm{ml})$ was not altered after $2 h$, but was significantly $(p<0.05, n=4)$ reduced to $77.0 \pm 3.3 \%$ of control after 20h of co-culture. Conclusions: This study provides the first demonstration that VCAM-1 is involved in the initial attachment of eosinophils to airway smooth muscle cells and that ICAM-1 is involved in their prolonged adhesion. It is possible that eosinophils, attached to smooth muscle cells via these molecules, directly modulate airway smooth muscle function in asthmatic airways.

Acknowledgments: We thank the donors and acknowledge the collaborative effort of the transplant team at St Vincent's Hospital. This study was supported by NHMRC and the Ramaciotti Foundation of NSW. 
INTRAPLEURAL FIBRINOLYTICS IN COMPLICATED PARAPNEUMONIC EFFUSIONS AND EMPYEMA- A SYSTEMATIC REVIEW.

R.J.Cameron, H.Davies

Background. Intrapleural fibrinoiysis has been used to treat complicated parapneumonic effusions and thoracic empyema for 50 years. Randomised controlled trials (RCT) addressing efficacy, safety and equivalent dosing have emerged only in the late 1990s. Objectives To conduct a systematic review of the literature concerning the benefit of adding intrapleural tibrinolytic therapy to intercostal tube drainage in the treatment of complicated parapneumonic effusions and empyema. Selection criteria. All studies were Randomised Controlled Trials. Participants. Patients $>14 \mathrm{y}$ with thoracic empyema or complicated parapneumonic effusions with no prior surgical intervention or trauma. Types of Interventions. 1/ Intrapleural fibrinolytics $v$ control. $2 /$ Intrapleural streptokinase vs. intrapleural urokinase. Data collection \& analysis. All identified RCTs were reviewed independently by two reviewers. Reviews were scored according to the Cochrane assessment of allocation concealment and the Jadad scale of methodological quality. Main results. One RCT directly comparing streptokinase and urokinase found both were equally effective in treatment outcomes, but streptokinase had a slightly higher non-fatal complication rate. Two RCTs compared streptokinase or urokinase vs normal saline control. There was significant improvement in length of hospital stay $(p=0.02)$, time to defervescence $(p=0.008)$, improvement in chest radiograph, treatment failure defined as death or requirement for surgery $(p=0.02)$ when the results were pooled, although individual study results did not necessarily show this. Significant complications attributable to therapy were not seen.

Conclusions. Intrapleural fibrinolytic therapy confers significant benefit when compared with normal saline control. The numbers in the RCTs are small and results of a larger 500 patient trial are awaited. Both agents are equally efficacious but streptokinase has a slightly higher non-fatal complication rate. Life-threatening complications are rare and were not seen in the RCTs. Evidence therefore suggests that intrapleural fibrinolysis can be considered as first-line therapy in these conditions. Issues regarding optimal dosing schedules and intrapleural fibrinolysis v early surgery are discussed and larger trials are awaited for this evidence.

DEVELOPMENT OF MOLECULAR DIAGNOSIS IN COMMUNITY ACQUIRED PNEUMONIA (CAP)

R Laing ${ }^{*}$ C Coles ${ }^{\#}$, J McWha", N Karulus ${ }^{\#}, S$ Chambers^, L Jennings ${ }^{+}, G$ Mills", R Cursons", W Slater" 1 Town^

Canterbury Respiratory Research Group*, Waikato Respiratory Research Group\#, Canterbury Health Laboratories ${ }^{+}$, Dept of Medicine, Christchurch School of Medicine^, New Zealand.

Aim: To assess the diagnostic yield of rapid molecular diagnostic techniques, for causative pathogens in CAP.

Methods: All patients over the age of 18 years admitted to Christchurch and Waikato hospitals between $26 / 07 / 99$ and $08 / 10 / 99$ with a principal diagnosis of CAP were prospectively enrolled in the study. Clinical samples were taken from each participant. All samples were processed for immediate traditional and new molecular diagnostic techniques. The remaining aliquots were frozen at $-80^{\circ} \mathrm{C}$ for subsequent molecular diagnostic testing. Participants were followed up at 6 weeks. Age and sex matched controls admitted with non respiratory conditions were identified and sampled to allow comparative testing of new molecular diagnostic techniques.

Results: 183 participants were enrolled with a mean age of 64 years, $53 \%$ female. $84(46 \%)$ had flu vaccination in the proceeding 12 months, however only $6(3.3 \%)$ received "Pneumovax". $54(30 \%)$ had received antibiotics prior to admission. Average length of hospital stay was 6.7 days with 8 (4.4\%) deaths within the 6 week follow up period. $9(5 \%)$ were admitted to ICU and $28(15 \%)$ had significant complications attributable to their CAP. Of the survivors, six week follow up occurred in $93 \%$. A microbial diagnosis was achieved for $92(50 \%)$ cases, $36(20 \%)$ St. pneumoniae, $29(16 \%), H$ influenzae, 24 (13\%) Influenza A, $10(5 \%)$ Adenovirus, $5(3 \%)$ Influenza B, 5 (3\%) RSV, 5 (3\%) Legionella spp, 4 (2\%) S. aureus, $2(1 \%)$ Mycoplasma pneumoniae and $5(3 \%)$ others. In $29(16 \%)$ cases multiple organisms were isolated. Legionella PCR has been performed for 86 cases, being positive in $9(10 \%)$, with no positives in the controls tested.

Conclusion: These are results from an ongoing 12 month study. The diagnostic yield from traditional techniques was $50 \%$. We have demonstrated an improved sensitivity for Legionella spp. through the application of Legionella PCR to samples from the same population, with no false positive results from an age and sex matched control population. We will go on to further explore the utility of new molecular techniques for the diagnosis of Legionella spp. and other common causative pathogens in CAP.
QUANTITATIVE ANALYSIS OF CMV PCR AND ANTIGENEMIA ARE COMPLEMENTARY IN PREDICTING DEVELOPMENT OF CMV DISEASE AND RESPONSE TO THERAPY POST TRANSPLANT

E. Gabbay, J. Flexman, R. Tarala, I. Kay, S. Palladino, R. Larbalestier, G. O'Driscoll, Cardiopulmonary Transplant Unit, Royal Perth Hospital, Australia

CMV disease is a major cause of morbidity in transplant recipients. The detection of CMV DNA by PCR or antigenemia may allow early detection of CMV infection but their predictive value is uncertain. Aim: To determine whether a quantitative assessment of CMV DNA in plasma by PCR (Roche CMV Monitor assay) and CMV antigenemia (pp65) could predict the development of CMV disease. Method: Serial weekly assays were performed in a blinded fashion on 16 thoracic organ recipients (9 Lung and 7 Heart) who were at risk for CMV disease (CMV Donor Positive / Recipient Negative or previous CMV disease). CMV disease was defined by tissue invasion with an appropriate clinical syndrome. Results: There were 10 episodes of CMV disease (pneumonitis 5, duodenitis 2, hepatitis 1 and colitis 2). Median (range) PCR (copies/ml) and antigenemia (positive cells/2 $\times 10^{5}$ leukocytes) in the weeks before and after disease are shown. Disease is at time-point zero. Significant differences $(p<0.05)$ between time points was determined by the Mann-Whitney test for paired samples.

\begin{tabular}{|l|l|l|l|l|}
\hline week & -2 & -1 & 0 & +1 \\
& pre-disease & $\begin{array}{l}+1 \\
\text { pre-disease }\end{array}$ & disease & $\begin{array}{l}3.6 \times 10^{4 \cdot} \dagger \\
\text { therapy }\end{array}$ \\
\hline PCR & 540 & $9700^{*}$ & $1.9 \times 10^{4}$ \\
& $(400-580)$ & $\left(590-3.5 \times 10^{4}\right)$ & $\left(1.7 \times 10^{4}-2 \times 10^{6}\right)$ & $\left(575-1 \times 10^{6}\right)$ \\
\hline antigenemia & $2(0-85)$ & $7(0-120)$ & $200(152-253)^{*} \dagger$ & $0(0-29) \ddagger$ \\
\hline
\end{tabular}

Significantly different from " time-point $-2, \uparrow$ time point -1 and $\neq$ disease. A 10 fold increase in CMV DNA detected by PCR was predictive of the subsequent development of CMV disease within 1 week [O.R. $=6.8$ (C.I. 4.6-11.2), $p<0.01]$. This was associated with a positive and negative predictive value of developing CMV disease of 90 and $87 \%$ respectively. In patients who developed CMV disease, an increase in CMV DNA (quantitative PCR) preceded the rise in antigenemia. However, in patients who improved clinically, a fall in CMV antigenemia preceded the fall in quantitative PCR. Conclusion: Quantitative analysis of CMV DNA by PCR appears to allow early prediction of disease whilst CMV antigenemia may be more useful in following response to therapy.

\section{FUNGAL INFECTIONS FOLLOWING LUNG TRANSPLANTATION M Tamm, M Malouf, A Glanville}

Heart Lung Transplant Unit, St Vincent's Hospital, Sydney, Australia Rejection and infection are the major contributors to morbidity and mortality after lung transplantation. Fungal infections are well recognised complications within the first few months after surgery but only few data are published in regard to the impact of late fungal infections. We analysed pulmonary fungal infections occurring later than 6 months after transplantation in 65 heart-lung (HLT), 105 single (SLT) and 108 bilateral (BLT) lung transplant recipients. Early pleural candida infection developed in 4 cases whereas late invasive candida infection was only found in two cases. Pulmonary nocardiosis was diagnosed in five patients later than 6 months after transplantation presenting as abscesses in three and empyema in another case. Three patients were successfully treated but in two the diagnosis was only made post mortem. Scedosporium apiospermium and Scedosporium prolificans was repeatedly documented in BAL fluid of 7 patients 11 to 58 months after transplantation. All 7 patients showed airway problems. Eradication of scedosporium infection proved difficult but this opportunistic infection did not disseminate under treatment with itraconazole and fluconazole. Aspergillus could be cultured from BAL of $26.2 \%$ HLT-, 28.6\% SLT- and $51.9 \%$ BLT recipients within the first few months after transplantation. Later than six months after surgery aspergillus was documented in $35.4 \%$ HLT-, $29.6 \%$ SLT and $22.2 \%$ BLT recipients. Invasive aspergillosis was most often associated with bronchiolitis obliterans but also developed in the native lung of $6 \mathrm{SLT}$ recipients.

Summary: late pulmonary fungal infections are associated with a considerable morbidity and mortality following lung transplantation, especially in patients with bronchiolitis obliterans. 
MYCOBACTERIUM KANSASII IN QUEENSLAND 1988-99

Rachel Thomson', John Armstrong', David Looke?

(1) Departments of Respiratory Medicine and (2) Infectious Diseases, Princess Alexandra Hospital (PAH), Brisbane. QLD 4102

Mycobacterium kansasii is the second most common pulmonary nontuberculous mycobacteria isolated in QLD. Aim: To review the clinical features, bacteriology and treatment of all pulmonary isolates of M.kansasii in QLD from 1988 - 99 . Of 71 isolates - 63 were pulmonary, 1 synovial, 1 soft tissue. Two HIV patients had M.kansasii in blood and sputum, 1 in blood and faeces. Results: Disease incidence rose from 0.073 (1988) to 0.376 (1998) per 100000 . Of the 65 pulmonary isolates, $49(75.4 \%)$ patients were notified as having clinically significant disease; 3 had probable disease and 3 possible disease. Median age of those with disease was 56 years (range 31-86), compared with 75 years (53-86) in those without disease ( $p=0.007)$. $77 \%$ were male. Predisposing conditions were seen in less than $30 \%$. Consolidation (41.7\%) and cavitation (36.7\%) were the most common Xray findings, predominantly in the upper lobes $(60 \%)$. Forty-five $(69.2 \%)$ patients were treated; 17 had documented side effects. Most regimens involved Ethambutol, Rifampicin, and Isoniazid (American Thoracic Society recommendations). Twenty-four patients $(56 \%$ of those treated) received Pyrazinamide ( 16 for more than 2 months). Mean duration of treatment was $12 \pm 6.6$ months (range $0.5-24$ ); $83 \%$ responded. Four patients died despite treatment ( 3 were intolerant of the medication); 4 died before treatment was initiated. There were no documented cases of relapse. In vitro sensitivities on 28 isolates showed all were sensitive to Rifampicin, 1 resistant to Ethambutol and all resistant to Isoniazid. Conclusions: Disease incidence has increased significantly. Treatment has been successful, though side effects common. $100 \%$ resistance to Isoniazid was seen, yet this drug is still included in treatment regimens. The absence of relapse in those treated for less than 18 months is supportive of shorter course therapy. Treatment of this disease in Australia should consider local experience and sensitivities.

Supported by a Princess Alexandra Hospital Clinical Research Fellowship and the Specialised Health Services TB Control Centre

Key words: mycobacteria, kansasii, nontuberculous

\section{0}

A RANDOMISED TRIAL OF HOME VS HOSPITAL INTRAVENOUS (IV) ANTIBIOTIC THERAPY IN ADULTS WITH INFECTIOUS DISEASES

Joanne Wolter, Ruth Cagney \& Joseph McCormack.

University Department of Medicine, Mater Adult Hospital, Brisbane.

Delivery of hospital care in the home is a rapidly growing industry favoured by patients, their families and the medical profession. Despite widespread adoption of home care services few randomised trials have compared health outcomes in the hospital and at home. We performed a prospective randomised trial of home versus hospital therapy in adults receiving IV antibiotics and compared clinical, quality of life (QOL) and cost outcomes. Methods: Consenting adults with - an infection requiring IV antibiotic therapy, compliance, stable disease, farmily support, telephone and transport were randornised to receive home care or complete their treatment in hospital. Where possible patients at home were taught to self-administer their antibiotics. QOL: Short Form 36 (SF-36) and Perceived Health Competence Scale (PHCS). Statistical analysis: unpaired $t$-tests, Mann-Whitney tests, ANOVA. Results: 128 patients were referred, 81 patients were randomised (39 home). Most common reason for non-inclusion was patient preference for one form of therapy over another (23 patients). Conditions treated included cellulitis, cystic fibrosis, pneumonia, UTI, wound infection, osteomyelitis, meningitis, endocarditis. 13 patients withdrew after randomisation, 7 because of randomisation to the hospital arm where an alternative home care option was offered. There were two unexpected readmissions in the home arm (patient request and CF not responding to therapy) and three further adverse events thought to be related to the study (IV site infection, PICC-line fracture and PICC related DVT). Preliminary analysis indicates average mean treatment duration was 18.1 days (SD 24) in the hospital arm and 14.2 days (SD 11.8) in the home arm $(p=0.38$ ). Mean duration of homebased care was 7.0 days (SD 5.2)

Conclusion: Recruitment to randomised trials of home versus hospital therapy is hampered by patient and staff expectations. Preliminary review of results suggests home IV therapy is well tolerated and is an appropriate treatment option for selected patients. Further clinical, QOL and cost outcomes will be presented.

Key words: home therapy, intravenous, quality of life, cost, infection Supported by: Mater Private Practice Fund, Roche Pharmaceuticals
THE TNF $\beta+250$ AND TNF $\alpha-308$ GENE POLYMORPHISMS ARE IMPORTANT IN COMMUNITY ACQUIRED PNEUMONIA(CAP). Waterer GW1 Quasney MW2 Z Zhang $Q^{2}$, Jones CB1, Wunderink RG1. IPulmonary and Critical Care Research, Methodist-Lebonheur Healthcare, Memphis. ${ }^{2}$ Department of Pediatrics, University of Tennessee, Memphis. Introduction: Clinically, CAP varies markedly even in similar patients with identical pathogens; genetic factors are likely to be important. The $T N F \beta+250$ and TNF $\alpha-308$ gene polymorphisms influence the outcome of some infectious diseases. We hypothesized that they would have an impact on CAP. Methods: A prospective cohort study of CAP. Septic shock(SS) was defined using ACCP-SCCM criteria. Type I respiratofy failure(T1RF) was defined as an $\mathrm{O}_{2}$ saturation on room air of $<90 \%$ with a normal $\mathrm{pCO}_{2}$. Genotype was determined using PCR amplification and restriction enzyme digestion. Genotype proportions are given in the order of AA/GAGG. Results: 169 patients were genotyped, 19 had SS, 45 had T1RF. The proportion of patients in each genotype developing SS were: TNF $\beta+250$ $0.18 / 0.07 / 0.11$ ( $p=0.08$ AA vs non AA); TNF $\alpha-308$ 0.20/0.09/0.11 ( $p=N S$ ) Carrying at least one AA genotype had an $18.6 \%$ risk of SS vs $7.3 \%$ $(p=0.04)$. GG homozygotes at both sites had only a $3.6 \%$ risk of SS. No subject was AA at both sites. T1RF in each TNFB+250 genotype was: $0.31 / 0.20 / 0.37(p=N S)$, however for T1RF in patients with no chronic respiratory disease it was $0.31 / 0.20 / 0.50$ ( $p=0.02$ for GG vs GA). Conclusions: The TNF $\beta+250$ and TNF $\alpha-308$ AA (hyper-secretor) genotypes are associated with SS and therefore these polymorphisms are important in the genetic variability of host response to CAP. The association between T1RF in patients without chronic lung disease and the TNF $\beta+250$ GG genotype (TNF $\alpha$ hypo-secretor) has important implications for immunotherapy in both CAP and sepsis, as well as for the definition of the systemic inflammatory response syndrome(SIRS) itself. Funding: The Athelstan and Amy Saw Medical Research Fellowship(GWW), the Methodist-Lebonheur Healthcare Foundation (GWW) and the Crippled Children's Foundation (MWQ).

Wednesday April 12 - Asthma \& Allergy SIG Orals (1500-1630)

DOES ENCASEMENT OF BEDDING IMPROVE ASTHMA IN ATOPIC ADULTS WITH ASTHMA?

S.C.Dharmage', M.Bailey', C.Wharton 1 , J.Raven², D.Cao3, J.Rolland ${ }^{3}$ F.Thien ${ }^{4}$, L.Light ${ }^{4}$, N.Freezer', E.H.Walters'2, M.Abramson 1 Departments of (1) Epidemiology \& Preventive Medicine. (2) Respiratory Medicine (3) Pathology \& Immunology (4) Allergy Asthma \& Clinical Immunology, Monash Medical School \& The Alfred Hospital, Prahran, 3181 and (5) Respiratory Meedicine, Monash Meedical Centre. Clayton 3168

The efficacy of house dust mite (HDM) avoidance in improving asthma control is controversial. Aim: To evaluate the impact of encasement of bedding with impermeable covers on asthma outcomes in HDM sensitised adults. Methods: A group of 33 young adults with current asthma and positive skin tests to HDM were recruited from Asthma Clinics. They were randomised into either an intervention group whose pillows, mattress and doonas were encased with impermeable covers, or a control group who received cotton covers. Before and 3 and 6 months after encasement, home visits were made to collect dust samples which were assayed for Der $p 1$. Clinical outcomes included quality of life, lung function, bronchial hyper-reactivity to methacholine and symptom/medication/peak flow diaries. Differences between groups were examined using $t$ tests and repeated measures analysis of variance. Results: Quality of life improved in both the intervention and control groups $(p=0.02$, 0.04 ), but there was no significant difference between groups. There was no significant change in $F E V_{1}, F V C$ or $\mathrm{PD}_{20}$. There were no significant differences between groups in daytime or nocturnal symptoms, requirements for reliever or preventive medication, morning or evening peak flows. Diurnal variability in peak flow was greater in the control group $(10.4 \% \vee 8.2 \%, p=.05)$, but the change over time did not differ between groups. Conclusions: Encasement of bedding alone is insufficient to produce worthwhile clinical improvements in asthma symptoms, medication requirements, lung function, bronchial hyperreactivity or quality of life in adults with asthma who are sensitised to HDM. Supported by the Victorian Department of Human Services. Key words: Asthma, encasement of bedding, house dust mites, epidemiology Nominations for awards: Nil 


\section{A METHOD FOR DETECTING AND MEASURING HDM ALLERGEN IN} THE UPPER AIRWAYS

Santos e Fonseca CMC., Rimmer J., Vanlaar C, Tovey ER, Salome C. Institute of Respiratory Medicine, University of Sydney, NSW 2050 HDM allergen has been related to asthma pathogenesis and in susceptible individuals may be related to severity or exacerbations of asthma. Although it is possible to measure environmental HDM allergen levels, we have no non invasive and sensitive method to measure the amount of allergen that reaches the airways. Aim: To develop methods to detect and measure Der $p 1$ in the upper airways. Methods: In 5 non-atopic subjects $0.2 \mathrm{ml}$ aqueous Der $p 1$ extract ( $3800 \mathrm{ng}$ ) was sprayed into a nostril on 2 different days. A nasal wash, using a squeeze bottle containing $10 \mathrm{ml}$ saline, was performed before allergen inhalation. Thirty seconds after the inhalation subjects gargled with $10 \mathrm{ml}$ saline, then performed a second nasal wash and nasal mucus was collected. Mucus was divided into liquid (n. mucus-L)and gel ( $n$. mucus $G$ ). Der $p$ I leveis in all samples were measured by ELISA assay, and the amount of allergen was expressed as a percentage of the dose delivered. Results: The table shows \% recovery in each sample on 2 test days (mean $\pm 95 \%$ confidence intervals).

\begin{tabular}{|l|l|l|l|l|}
\hline & gargle & n. wash & n. mucus- L & n. mucus-G \\
\hline Test 1 & $1.63 \pm 2.1 \%$ & $5.18 \pm 3.6 \%$ & $4.64 \pm 3.8 \%$ & $1.72 \pm 1.49 \%$ \\
\hline Test 2 & $0.24 \pm 0.47 \%$ & $2.33 \pm 2.3 \%$ & $4.3 \pm 3.3 \%$ & $2.18 \pm 1.42 \%$ \\
\hline
\end{tabular}

Conclusion: It is possible to detect Der $p 1$ in secretions from the upper airways following allergen administration. The allergen is primarily found in the nasal cavity, detectable in the mucus or the nasal wash, and very little passes through to the pharynx to be detected in the gargle. This method could be a useful new tool to examine amount of allergen that reaches the airways under varying conditions.

Key words: HDM, Der $p 1$ allergen, respiratory secretions, non invasive method

Study support: FAPESP - Brazil, Institute of Respiratory Medicine Australia

\section{THE ROLE OF THE SULFITE ADDITIVES IN WINE-INDUCED ASTHMA}

Hassan Vally \& Philip J. Thompson.

Asthma \& Allergy Research Institute, \& Department of Medicine. University of Western, Nedlands. Western Australia.

Although the sulfite additives have been implicated as a major cause of wine-induced asthma, direct evidence for this is limited. Aims and Methods: The aims of this study were to identify asthmatics exquisitely sensitive to the sulfite additives in wine using a single dose sulfited wine challenge protocol, and to describe the dose response characteristics of these responses using a double blind placebo controlled challenge model. In addition, the efficacy of a cumulative dose sulfited-wine challenge protocol to identify asthmatics sensitive to the sulfite additives was also assessed. Results: Four out of 23 self-reporting wine-sensitive asthmatics were found to respond to sulfite additives in wine using the single dose challenge protocol. In the double blind dose-response study, all of these asthmatics exhibited a significant fall in $\mathrm{FEV}_{1}$ ( $>15 \%$ from baseline) following challenge with wine containing 300 ppm sulfite, but did not respond to wines containing 20,75 or $150 \mathrm{ppm}$ sulfite. Asthmatic responses were maximal at $5 \mathrm{~min}$, with an average decline in FEV ${ }_{1}$ of $28.7 \pm 13 \%$, and took between 15 and 60 min to return to baseline levels. In the cumulative dose-response study, the responsiveness of 12 asthmatics providing a history suggesting a sensitivity to the sulfite additives in wine was compared with the response of 6 asthmatics reporting no sensitivities to these drinks. No significant difference was observed in any of the lung function parameters measured $\left(\mathrm{FEV}_{1}, \mathrm{PEF}_{1} \mathrm{FEF}_{25-75}\right)$ in these 2 groups. Conclusions: These results suggest that only a small number of wine-sensitive asthmatics respond to a single dose challenge with sulfitedwine under laboratory conditions and that cofactors may be playing a role in the sensitivity of many asthmatics to wine. A cumulative dose challenge protocol did not increase the sensitivity of our test for reactivity to the sulfites in wine.

Acknowledgements: Wines for these studies were kindly donated by Hardys BRL

Key words: Asthma, wine, sulfites.

\section{REDUCTION IN AIRBORNE HOUSE DUST MITE ALLERGEN AFTER} OCCLUSIVE COVERING OF BEDDING

\author{
R. Siebers, J. Lane, M. Sakaguchi", P. Fitzharris, J. Crane.
}

Wellington Asthma Research Group, Wellington School of Medicine, Wellington, New Zealand: and National Institute of Health-', Tokyo. Japan.

Mite occlusive bedding covers are recommended to reduce house dust mite (HDM) allergen exposure for HDM sensitised individuals with allergic disease. As inhaled HDM allergen is more relevant than HDM allergen levels in settled dust, we have studied whether occlusive bedding covering reduces the airborne HDM allergen, Der $p 1$ in bedrooms.

We obtained dust samples by vacuuming $\left(1 \mathrm{~m}^{2} / \mathrm{min}\right)$ from pillows, duvets, and mattresses of 12 subjects. Additionally, air was sampled for $8 \mathrm{hr} / \mathrm{hight}$ for 7 nights with a personal sampler (Sibata, Japan) at $1 \mathrm{~L} / \mathrm{min}$. Pillows, duvets, and mattresses were then covered with occlusive covers (Alprotec, UK) for 7 days, and during this time air was sampled as described above. After the 7 days, dust samples were obtained as described above directly from the surface of the bedding covers. Der $p 1$ in dust samples was measured by double-monoclonal antibody ELISA, and in the air samples by an extremely sensitive fluorimetric ELISA. Der $p 1$ levels are expressed as geometric mean ( $95 \%$ confidence intervals), bedding samples as $\mathrm{ug} / \mathrm{m}^{2}$, and airborne samples as $\mathrm{pg} / \mathrm{m}^{3}$. Statistical analysis was by paired $\mathrm{t}$-test. Der $p 1$ levels decreased after covering pillows (from $0.47 \mathrm{ug} / \mathrm{m}^{2}, 0.20-1.10$ to $0.09 \mathrm{ug} / \mathrm{m}^{2}, 0.05-0.17, \mathrm{p}=0.0021$ ), duvets (from $1.15 \mathrm{ug} / \mathrm{m}^{2}, 0.30-4.43$, to $0.07 \mathrm{ug} / \mathrm{m}^{2}, 0.03-0.16, \mathrm{p}=0.0001$ ), and mattresses (from $26.0 \mathrm{ug} / \mathrm{m}^{2}, 5.9$ 114.8 , to $\left.0.34 \mathrm{ug} / \mathrm{m}^{2}, 0.08-1.40, p=0.0004\right)$. Airborne Der $p 1$ decreased from $102.1 \mathrm{pg} / \mathrm{m}^{3}$ (45.6-228.6), to $17.5 \mathrm{pg} / \mathrm{m}^{3}(7.2-42.7), p=0.0063$. This study is the first to show that, concomitant with a reduction in bedding Der $p 1$ levels, airborne Der $p 1$ levels decreased approximately six-fold after occlusive covering of bedding for 7 days.

Supported by a grant from the Health Research Council of New Zealand

SETTLING DER P 1 IS NOT AFFECTED BY ALLERGEN CONTROL IN BEDS Vanlaar $\mathrm{CH}^{1}$, Mihrshahi $\mathrm{S}^{2}$, Peat $\mathrm{JK}^{2}$, Marks $\mathrm{GB}^{1,3}$, Forbes $\mathrm{S}^{2}$, Vukasin $\mathrm{N}^{2}$, Wainwright $\mathrm{C}^{3}$, Krause W3, Prater $\mathrm{G}^{2}$, Mellis $\mathrm{C}^{2}$, Tovey ER' 1 Institute of Respiratory Medicine, NSW 2050; ${ }^{2}$ Clinical Epidemiology, New Children's Hospital, NSW 2124: ${ }^{\text {DDept. Respiratory Medicine. Liverpool }}$ Hospital. NSW 2170

Rationale: Petri dishes may be used to collect settling dust to provide a simple estimate of ambient mite aeroallergen exposure over time. We measured aeroallergen near the beds of control and intervention groups involved in a study controlling mite allergen in beds and compared this to reservoir levels in those beds.

Methods: Settling dust was collected in Petri dishes placed adjacent to beds that had been fitted with occlusive mattress and pillow covers and where all bedding was laundered with an acaricidal additive. Dishes were also placed next to control beds where no intervention had been implemented. Reservoir bed dust was collected by vacuum sampling at the time dishes were opened (zero weeks) and eight weeks later. Dishes were left open for four to eight weeks. Der $p 1$ was extracted from reservoir and settling dust and measured by ELISA. Results: Geometric mean (GM) and median values are presented

\begin{tabular}{|c|c|c|c|}
\hline Der $p 1$ measure & Active & Control & p value \\
\hline Median Petri dish ( $\mathrm{ng} / \mathrm{m}^{2} /$ day) & $15.38(n=79)$ & $15.29(n=79)$ & $0.7477^{\star}$ \\
\hline GM in bed $(\mu g / g) @ 0$ weeks & $2.56(n=73)$ & $3.36(n=71)$ & $0.3361^{\prime \prime}$ \\
\hline GM in bed $(\mu \mathrm{g} / \mathrm{g}) 8 \mathrm{~s}$ weeks & $3.52(n=75)$ & $7.16(n=75)$ & $0.0017^{*}$ \\
\hline Median diff (wks 8-0) in total $(\mu \mathrm{g})$ & $0.022(n=77)$ & $0.044(n=76)$ & $0.5719^{*}$ \\
\hline
\end{tabular}

"Mann-Whitney $U$ test "* Independent samples t test "** Median test Correlations (Spearman's) of Petri dish settling rate with Der $p 1$ concen. in beds: Week 0 tho $=0.23(p=0.0057)$; Week 8 tho $=0.27(p=0.0008)$

Petri dish with rate of change in total allergen: rho $=0.10(p=0.2286)$ Conclusions: The bed intervention did not result in a lower rate of Der $p 1$ allergen settling in Petri dishes kept adjacent to beds despite intervention beds having less total allergen and a lower concentration of allergen in dust. Petri dish Der $p 1$ allergen levels showed a weak but significant correlation with bed reservoir levels but not the rate of total allergen change in beds. The allergen content of settling airborne dust may be affected by sources other than the bed. (Supported by the NHMRC) Key words: allergen control, Petri dish, aeroallergen 
REPORTED FOOD ALLERGY IS OVERESTIMATED

Rosalie Woods ${ }^{1}$, Rachel Stoney', Michael Bailey', Joan Raven2, Frank Thien ${ }^{3}$, Michael Abramson ${ }^{1}$, E. Haydn Walters ${ }^{2}$ (1) Epidemiology \& Preventive Medicine (2) Respiratory Medicine (3) Allergy \& Clinical Immunolegy, Monash Medical School and Affred Hospital, VIC 3181

We have previously found that young adults perceive food intolerance and allergies as common, however IgE mediated food allergy is deemed to be uncommon. Aim: To determine how much of perceived food allergy/intolerance was due to $\mathrm{lgE}$ mediated food allergy, as defined by skin prick tests (SPT). Methods: In 1998, 457 young adults (26-50 years) underwent SPT to five common food allergens (cow's milk, peanut mix, egg white, shrimp and wheat) and were asked whether they had ever suffered any food "illness/trouble", and if so to list such food(s). A positive SPT was defined as wheal formation of $\geq 3 \mathrm{~mm}$ diameter. Cohen's kappa ( $k$ ) was used to assess the agreement between SPT and self-reported reactions to food(s) which contain the allergen of interest. Results: 62 participants had a positive SPT to at least one food allergen. The table below summarises the main results. $A \times$ value of $<0.2=$ poor agreement.

\begin{tabular}{|l|c|c|c|c|}
\hline $\begin{array}{l}\text { Food } \\
\text { allergen }\end{array}$ & $\begin{array}{c}\text { SPT+ } \\
\text { (n) }\end{array}$ & $\begin{array}{c}\text { 'Illness/trouble' } \\
\text { wlth food (n) }\end{array}$ & $\begin{array}{c}\text { SPT + \& food } \\
\text { 'Illness/trouble' } \\
\text { (n) }\end{array}$ & $\mathbf{K}$ \\
\hline Cow's milk & 4 & 29 & 0 & -0.016 \\
\hline Peanut mix & 28 & 8 & 4 & 0.200 \\
\hline Shrimp & 19 & 20 & 6 & 0.277 \\
\hline Egg white & 9 & 9 & 1 & 0.093 \\
\hline Wheat & 13 & $\mathbf{9}$ & 0 & -0.024 \\
\hline
\end{tabular}

Conclusions: There was little agreement between self-reported "illness/troublen to food(s) known to contain the food allergen of interest, and positive SPT, suggesting that most reactions are not due to IgE mediated food allergy.

Supported by the NHMRC

Key words: food intolerance, food allergy, skin prick test, epidemiology Nominations for awards: $\mathrm{Nil}$

CPAP DOES NOT ABOLISH INTRINSIC POSITIVE END-EXPIRATORY PRESSURE (IPEEP) IN SEVERE STABLE COPD.

F O'Donoghue1, P Catcheside1, RS Orr'1, A Jordan'1, A Bersten², \& RD McEvoy' 1) Sleep Disorders Unit, Daw Park Repatriation Genera Hospital, SA 5041 2) Department of Critical Care Mecdicine. Flinders Medical Centre, SA 5041

Introduction: IPEEP may contribute to chronic respiratory failure in COPD, constituting an inspiratory threshold load (ITL) on muscles already at a severe mechanical disadvantage. Extrinsic PEEP has been advocated to relieve the ITL caused by iPEEP. We sought to document the effect of incrementally increasing extrinsic PEEP (as CPAP) on ITL during wakefulness. Methods: Nine patients with severe stable COPD (mean FEV, $30.7 \%$ pred) were studied. CPAP was increased in $1 \mathrm{cmH}_{2} \mathrm{O}$ increments to a maximum of $10 \mathrm{cmH}_{2} \mathrm{O}$. At each pressure we measured: i) iPEEP as the change in oesophageal pressure ( $\triangle$ Poes) from the onset of inspiratory effort to the start of flow ii)Change in transdiaphragmatic pressure ( $\Delta \mathrm{Pdi}$ ) over the same interval. iii) Pressure-time product (PTP) for the respiratory muscles. iv) End-expiratory lung volume (EELV). Results: In 8 subjects, $\triangle$ Pdi closely matched $\triangle$ Poes, indicating minimal contribution of abdominal expiratory muscles to measured PPEEP. In these 8 , mean ( \pm SEM) baseline iPEEP was $2.86\{0.61\} \mathrm{cmH}_{2} \mathrm{O}$. CPAP significantly reduced but did not abolish measured ITL. Mean minimum ITL was $0.96(0.15) \mathrm{cmH}_{2} \mathrm{O}(p<0.05)$. PTP per litre ventilation reduced from $5.84(1.03)$ to $2.81(0.42) \mathrm{cmH}_{2} \mathrm{O} / \mathrm{L}$ without a change in minute ventilation, but at the expense of progressively increasing lung volume, to a maximum of $0.58(0.11) \mathrm{L}$ above baseline.Conclusions: In severe stable COPD it is not possible to abolish the ITL due to IPEEP using CPAP, Inspiratory muscle pressure output is reduced but there is progressive hyperinflation. Possible reasons include i) iPEEP is not due to dynamic airway compression, but rather fixed airflow obstruction ii) marked inhomogeneity in severity of dynamic airway compression prevents complete counterbalancing of IPEEP.

Supported by the NHMRC and Daw Park Research Foundation

Key words: COPD, intrinsic PEEP, CPAP.

Nominated for Awards: John Read prize.
TREATMENT OF OBSTRUCTIVE SLEEP APNOEEA WITH NOCTURNAL CPAP INCREASES AWAKE ALVEOLAR VENTILATION AND DECREASES VENTILATION-PERFUSION $\left(V_{A} / Q\right)$ INEQUALITY. T D Robinson, C E Sullivan and I H Young Department of Respiratory Medicine, Royal Prince Alfred Hospital, Sydney. NSW, 2213 Some patients with obstructive sleep apnoea (OSA) have daytime hypoxia in the absence of significant lung disease. Treatment of OSA with continuous positive airway pressure (CPAP) can partly reverse this hypoxia: the mechanisms of improvement are unclear. We have performed detailed gas exchange studies using the multiple inert gas elimination technique (MIGET) on 4 patients with OSA, 3 with awake hypoxia and eucapnia and 1 with awake hypoxia and hypercapnia (obesity hypoventilation syndrome [OHS]), at baseline and following 4 months of treatment with CPAP (3 patients) or bilevel positive pressure (1 patient). The patients were obese, BMI $48(10$ $\mathrm{kg} / \mathrm{m}^{2}$ (SD) with FEV 1 2.2(0.2 L (68(5\% predicted) and $\mathrm{FEV} / 1 \mathrm{FVC} 82(5 \%$ with significant hypoxia, $\mathrm{PaO}_{2} 52\left(11 \mathrm{mmHg}\right.$ and $\mathrm{PaCO}_{2} 47(8 \mathrm{mmHg}$. At baseline, the MIGET measure of perfusion to low $V_{A} / Q$ units $(\log S D Q)$ was increased at $0.70(0.35$ (normal $<6.0)$, with increased shunt in 3 patients (3.3(3.3\%). The MIGET measure of ventilation to high $V_{A} / Q$ units $(\log S D V)$ was normal $\left(0.54(0.11)\right.$. After 4 months of treatment, $\mathrm{PaO}_{2}$ had increased to $6019 \mathrm{mmHg}\left(\mathrm{p}=0.08\right.$, paired t-test) and $\mathrm{PaCO}_{2}$ had fallen to $42(8 \mathrm{mmHg}$ $(p<0.01)$. Alveolar ventilation $\left(V_{A}\right)$ had increased in all, $7.3(1.1 \mathrm{~L} / \mathrm{min}$ to $8.7(0.8 \mathrm{~L} / \mathrm{min}(p<0.05)$ and $\log \mathrm{SD} Q$ and shunt had both fallen to $0.62(0.38$ $(p<0.05)$ and $1.6\left(1.9 \%(p=0.08)\right.$ respectively. The increase in $\mathrm{PaO}_{2}$ was due to both reduced $V_{A} / Q$ inequality $(64 \%)$ and increased $V_{A}$. Treatment of OSA and $O H S$ increases awake $V_{A}$, reduces the perfusion to low $V_{A} / Q$ units and decreases intrapulmonary shunt. This suggests that patients with OSA (even without OHS) have chronic awake hypoventilation and that sleep-disordered breathing results in awake $V_{A} / Q$ inequality. Treatment with nocturnal CPAP partly reverses these abnormalities.

Supported by the NH\&MRC

Key words: OSA, MIGET, CPAP, hypoxia

Nominations for Awards: TSANZ/ALF John Read Prize Physiological Research

REPEATABILITY AND NORMALISATION OF DIAPHRAGM ELECTROMYOGRAM (EMG) SIGNAL STRENGTH IN HEALTHY SUBJECTS

B. Singh1,3, J.A. Panizza1, P.J. Drury ${ }^{2}$, K.E. Finucane ${ }^{1}$

Departments of 1 Pulmonary Physiology and 2Radiology, Sir Charles Gairdner

Hospital, Western Australia and ${ }^{3}$ Physiology, University of Western Australia.

The root mean square of the diaphragm EMG signal (RMSdi) during inspiration has been used to quantify activation of the diaphragm during tidal breathing (Sinderby et al JAP 1998;85:2146-58). To examine the RMSdi response to hypercapneic ventilatory stimulation, its repeatability, and methods of normalisation, we measured breath-by-breath RMSdi, tidal volume, duration of respiratory cycle (Ttot), and end-tidal $\mathrm{PCO}_{2}$ in four healthy subjects (age [mean $\pm \mathrm{SD}$ ] $44.8 \pm 13.5 \mathrm{yrs}$, BMI $25.1 \pm 1.0 \mathrm{~kg} / \mathrm{m}^{2}$ ) on two occasions during steady-state ventilation at seven levels of $\mathrm{FiCO}_{2}$ between 0 and 0.08 presented in random order. RMSdi was measured using a multi-electrode oesophageal catheter and controlled for signal contamination and diaphragm position. We also measured RMSdi obtained during a slow inspiration from FRC to TLC (RMSdimax), electrocardiogram $\mathrm{R}$ wave amplitude at the oesophageal electrode pair closest to the diaphragm and thickness of the costal diaphragm by ultrasound. We found that (a) the coefficient of variation of RMSdi over 10 breaths at peak ventilatory stimulation $\left(\mathrm{FiCO}_{2} 0.08\right)$ ranged from 0.07 to 0.15 , and this coefficient improved only minimally when a greater number of breaths were analysed, (b) RMSdi increased progressively with $\mathrm{FiCO}_{2}$ in all subjects, (c) the repeatability of RMSdi improved after normalisation with either RMSdimax or R wave amplitude, (d) differences in RMSdi between the subjects were eliminated after normalising with RMSdimax and $T$ tot $(p=$ 0.10 , ANOVA) and (e) RMSdi was not proportional to thickness of the costal diaphragm. We conclude that neural drive to the diaphragm in healthy individuals can be measured using the mean RMSdi over 10 breaths during steady state ventilation, and can be normalised by dividing it by the RMSdi during a breath from FRC to TLC and the duration of the respiratory cycle.

Supported by NHMRC, Medical Research Fund of WA and SCGH Research Fund. 
UPPER AIRWAY EXTRALUMINAL TISSUE PRESSURE IN RABBITS Kristina Kairaitis, Sarah R. Garlick, Jason P. Kirkness, Terence C. Amis and John R. Wheatley.

Department of Respiratory Medicine. Westmead Hospital and University of Sydney, Westmead. NSW 2145

Pharyngeal patency depends upon the balance of forces acting across the upper airway walls. While the influence of intraluminal pressure on upper airway patency has been studied extensively there is little information describing upper airway extraluminal tissue pressure (ETP). Methods: We studied 7 adult, male, supine, anaesthetised, spontaneously breathing, New Zealand White rabbits. ETP was measured with a pressure transducer tipped catheter (MPC 500, Millar) surgically inserted into the tissues surrounding the lateral pharyngeal wall. Respiratory airflow was measured via a mask and pneumotachograph. The effect of head positioning and mouth opening on ETP was examined. A tracheostomy was then performed and the effect of caudal traction applied to the trachea (quantified using a force transducer attached to the cranial segment) was studied. Results: During spontaneous breathing and with the head positioned at $30^{\circ}$ to the horizontal, mean ETP was positive in all rabbits (5.6 (1.7 SEM), range 1.2-13.5 $\mathrm{cm} \mathrm{H}_{2} \mathrm{O}$ ) and varied from 6.5 (1.7) $\mathrm{cmH}_{2} \mathrm{O}$ to $5.1(1.8) \mathrm{cmH}_{2} \mathrm{O}$ with the same frequency as respiration. There was no signiticant change in ETP with head flexion from $30^{\circ}$ to $50^{\circ}$. However, further flexion to $7^{\circ}$ resulted in a significant increase in ETP to $7.7(1.9) \mathrm{cm} \mathrm{H}_{2} \mathrm{O}$ (ANOVA, p<0.05 compared to $30^{\circ}$ and $50^{\circ}$ ). Mouth opening (no mask, head position of $70^{\circ}$ ) to $1 \mathrm{~cm}$ (between the upper and lower incisor teeth) did not change mean ETP, however, mouth opening to $2 \mathrm{~cm}$ increased mean ETP to 10.1 (2.5) $\mathrm{cm} \mathrm{H} \mathrm{H}_{2} \mathrm{O}$ (p<0.05 ANOVA, compared with mouth closed). No significant effect of mouth opening was observed with other head positions (p<0.05). Following tracheostomy, caudal displacement of the trachea with a mean force of 155.4 (35.4) $\mathrm{g}$ resulted in a significant decrease in ETP from 11.6 (6.4) to $7.7(5.5) \mathrm{cm} \mathrm{H}_{2} \mathrm{O}(\mathrm{n}=6, \mathrm{p}<0.05$ Wilcoxon signed rank). Conclusions: In rabbits, ETP is positive and thus exerts a collapsing force on the upper airway. This force is increased by head flexion and mouth opening, and decreased by caudal traction on the trachea.

Supported by: NH\&MRC

Key words: Upper Airway, Extraluminal tissue pressure

Nominations for Awards: John Read Prize

Wednesday April 12 - Cystic Fibrosis SIG Orals (1500-1630)

SPUTUM CYTOLOGY AND IL-8 IN CYSTIC FIBROSIS (CF) INPATIENTS

Dakin $\mathrm{CJ}^{1}, 2$, Henry $\mathrm{R}^{1,2}$, Wang $\mathrm{H}^{2}$, Morton $\mathrm{J}^{1,2}$.

1. Respiratory Medicine. Sydney Children's Hospital. Randwick. 2031.

2. School of Paediatrics, UNSW, Randwick, 2031.

There is considerable current interest in measuring inflammatory cytokines in CF respiratory secretions. In CF infants, IL-8 has been shown to correlate with infection. Small studies on CF sputum have failed to show similar correlations. The aim was to study the relationship between duration of treatment, lung function, sputum cytology and IL-8 in CF inpatients (representing a relatively homogeneous group of those with bronchiectasis) Methods: Prospective standardised collection of spontaneous sputum samples at least 6 days apart during admission for pulmonary exacerbation. FEV ${ }_{1}$ and FVC were recorded. Sputum processing was selective, with dispersal by the three enzyme technique. IL-8 kit assay. Results: 26 admissions of 19 patients, 9 boys, with mean age 13.4 years (6-19 years). Mean of 10 days between data points (range 7-34). FEV , mean at admission $58 \%$ predicted $(27-97 \%)$ and discharge $67 \%$ (29-99\%). Sputum total cell count (TCC) mean fell non-significantly during admission from 32 to $20 \times 10^{6} / \mathrm{ml}(+/-32 s d)$. IL-8 levels were all very high, but showed no change over admission (mean 90, range 30-159 $\mathrm{ng} / \mathrm{ml})$. IL-8 level correlated with sputum TCC $(0.49, p=0.005)$, neutrophils $(0.47, p=0.016)$ and macrophages $(-0.42, p=0.028)$, however $I L-8$ values for $42 \%$ of the samples were extrapolated from the standard curve. IL-8 did not correlate with FEV $V_{1}$ or FVC. As previously shown, TCC correlated with $\operatorname{FEV}_{1}(-0.43, p=0.001)$ and FVC $(-0.48, p=0.0001)$. Conclusion: In $C F$ inpatients with established inflammation sputum IL-8 levels were very high. They did correlate in the expected directions with cell counts, but not with pulmonary function or inhospital treatment. In contrast, sputum cytology and puimonary function were shown to correlate in the same sputum samples.

Key words: Cystic fibrosis, sputum, cytokines, cytology Nominations for Awards: Nil.

\section{ASYMPTOMATIC OBSTRUCTIVE SLEEP APNOEA CAUSES} NEUROBEHAVIOURAL DYSFUNCTION

Maree Barnes', Natalie Tarquinio', Siobhan Banks², Krystyne Camp1, Andrew Kenner ${ }^{2}$, R. Douglas McEvoy² \& Rob Pierce'1.

(1) Department of Respiratory Medicine, Austin and Repatriation Medical Centre. Victoria 3084 and (2) Sleep Disorders Unit. Daw Park Repatriation Hospital. South Australia 5041

Introduction Completely asymptomatic patients may have sleep disordered breathing (SDB), but the severity of this and how it is related to daytime function is unclear. Aim To assess the incidence of SDB and neurobehavioural function in asymptomatic subjects. Methods As part of a study looking at neurobehavioural function in patients with obstructive sleep apnoea, 23 subjects were recruited as normal controls. A standard script was used to eliminate those who had any symptoms of SDB, including snoring and breathing problems while asleep, and those who had any psychiatric or other medical problems. These subjects then underwent overnight polysomnography, a Maintenance of Wakefulness Test (MWT) and neurobehavioural assessment. Results The 18 males and 5 females had a mean (SD) age of 47.3 (8.1)

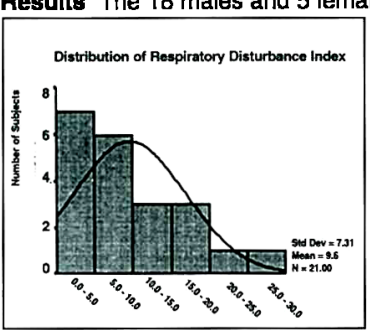

years, body mass index was 27.6 (3.4) $\mathrm{kg} / \mathrm{m}^{2}$ and abdominal:gluteal ratio was 0.89 (0.05). The mean (SD) respiratory disturbance index (RDI) was 9.6 (7.3), range $0.7-28.4$ and the mean (SD) arousal index (Al) was 14.8 (8.3). Mean (SD) MWT sleep latency was 37.7 (4.4) minutes and the Epworth Sleepiness Scale (ESS)(SD) score was $5.9(2.8) ; 17$ of 23 subjects had a normal ESS and MWT. Neurobehavioural assessment was abnormal in up to 5 patients in all areas tested, except verbal fluency (COWAT)

which had a reduced score in 10 subjects $(43.5 \%)$. There was no statistically significant correlation between the RDI and neurobehavioural results; there was a significant correlation between $R D I$ and abdominal:gluteal ratio $(R=0.50$, $p=0.04$ ), but not BMI. The MWT score correlated significantly with the Psychomotor Vigilance Task reaction time $(R=0.50, p=0.03)$ but not with the ESS score $(R=0.04, p=0.87)$. The ESS correlated significantly with the digit symbol substitution task $(R=0.42, p=0.05)$, a test of general cognitive ability. Conclusions 1 . Subjects who are screened to exclude symptoms of sleep disordered breathing may have mild to moderate obstructive sleep apnoea. 2. There is no statistically significant correlation between RDI and neurobehavioural dysfunction in these patients. 3. Daytime sleepiness correlates with vigilance and general cognition.

Supported by the NH\&MRC and the University of Melbourne

Key words: Obstructive sleep apnoea, neurobehavioural dysfunction

PREDICTING OXYGEN DESATURATION DURING SLEEP IN PATIENTS WITH CYSTIC FIBROSIS

Milross MA, Piper AJ, Norman M, Grunstein RR, Sullivan CE, Bye PTP. Respiratory Medicine, Royal Prince Alfred Hospital, Sydney, 2050, Australia Previous studies have suggested that an awake resting oxyhaemoglobin saturation $\left(\mathrm{SpO}_{2}\right)$ lower than $94 \%$ and an $\mathrm{FEV}_{1}<65 \%$ predicted are predictive of nocturnal desaturation in patients with cystic fibrosis (CF). This study was performed to evaluate predictors of sleep disordered breathing. Methods: Full dlagnostic sleep studies were performed in 21 patients with $\mathrm{CF}$ selected with an FEV $1<65 \%$ predicted who were in a stable clinical condition. Spirometry, lung volumes, and maximal inspiratory and expiratory pressures were measured. Arterial blood gas (ABG) measurements were taken in the afternoon and immediately upon awakening. Results: Patients were aged $26 \pm 5.6$ (mean $\pm \mathrm{SD}$ ) years, BMl $20+2 \mathrm{~kg} / \mathrm{m}^{2}, \mathrm{FEV}, 33 \pm 9 \%$ predicted, RV/TLC $58 \pm 10 \%$, awake $\mathrm{PaO}_{2} 67 \pm 8 \mathrm{mmHg}$ and $\mathrm{PaCO}_{2} 44 \pm 5 \mathrm{mmHg}$. Twelve out of 21 patients spent $>10 \%$ of total sleep time (TST) with $\mathrm{SpO}_{2} \leq 90 \%$, whereas 15 out of 21 patients spent $>10 \%$ of REM sleep time with $\mathrm{SpO}_{2} \leq 90 \%$. Three of 8 patients with an awake resting $\mathrm{SpO}_{2} \geq 94 \%$ had a TST average minimum $\mathrm{SpO}_{2}$ (the average of the minimum $\mathrm{SpO}_{2}$ per 30 second epoch of sleep) $<90 \%$ and 11 of 13 patients with resting $\mathrm{SpO}_{2}<94 \%$ had a TST average minimum $\mathrm{SpO}_{2}<90 \%$. All 21 patients had an absolute minimum sleep $\mathrm{SpO}_{2} \leq 90 \%$. Both the minimum sleep $\mathrm{SpO}_{2}$ and TST average minimum $\mathrm{SpO}_{2}$ correlated with awake resting $\mathrm{SpO}_{2}(r=0.76, p<0.0001 ; r=0.72, p<0.001)$ and $\mathrm{PaO}_{2}(r=0.78$, $p<0.0001$ both). FEV $1 \%$ predicted and Plmax \% predicted had the strongest correlation of the lung function parameters with minimum $\mathrm{SpO}_{2}$ during sleep $(r=0.51, p<0.05 ; r=0.47, p<0.05)$. No relationship was seen between nocturnal oxyhaemoglobin desaturation and lung volumes. A significant difference was seen in $\mathrm{PaCO}_{2}$ from the evening $(44 \pm 5 \mathrm{mmHg})$ to the morning $(46 \pm 6 \mathrm{mmHg})$ ABG measurement $(p<0.05)$. Conclusion: The significantly elevated morning $\mathrm{PaCO}_{2}$ is indirect evidence for hypoventilation in sleep. Awake $\mathrm{PaO}_{2}$, resting $\mathrm{SpO}_{21} \mathrm{FEV}_{1} \%$ predicted and PImax \% predicted correlate with minimum sleep $\mathrm{SpO}_{2}$. Normal awake resting $\mathrm{SpO}_{2}$ in $\mathrm{CF}$ does not exclude clinically significant desaturation during sleep. Further studies of the prevalence of sleep disordered breathing in patients with CF and mild lung disease are indicated. Grant Support: NHMRC Nominatlon for awards: No

Key words: cystic fibrosis, sleep, hypoventilation. 
RESPIRATORY SYMPTOMS, AIRWAY INFECTION AND LUNG FUNCTION IN INFANTS WITH CYSTIC FIBROSIS

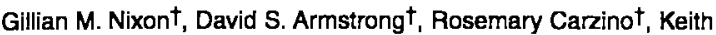
Grimwood ${ }_{\text {, Colin F. Robertson }}$.

IDepartment of Respiratory Medicine. Boyal Children's Hospital, Victoria 3052: \pm Department of Paediatrics. Wellington Clinical School. University of Otago. Wellington, New Zealand.

Airway infection, inflammation and respiratory symptoms may occur in infants with cystic fibrosis (CF) from the first few months of life. To explore the relationship between airway infection, lung function and respiratory symptoms, we conducted a cross-sectional study in 41 children with CF (median age $17 \mathrm{mo}$ ) who had bronchial lavage and lung function measurement under general anaesthesia. Forced expiratory volume in half a second $\left(\mathrm{FEV}_{0.5}\right)$ was determined by the raised volume rapid thoracic compression technique. A colony count $\geq 10^{5} \mathrm{CFU} / \mathrm{ml}$ bronchial lavage fluid and/or a differential neutrophil count $>50 \%$ was considered positive for airway infection/ inflammation. A healthy control group ( $n=8$, aged 5-32 mo) had lung function testing prior to elective surgery. Results: $18 / 41$ (44\%) children had airway infection, $56 \%$ of whom were asymptomatic. Conversely, $7 / 23(30 \%)$ infants without airway infection were symptomatic. Mean FEV 0.5 $(95 \% \mathrm{Cl})$ adjusted by analysis of covariance for height for those with and without infection was $215 \mathrm{ml}(187,243)$ and $244 \mathrm{ml}(219,269)$ respectively. Mean FEV 0.5 for the control group was $280 \mathrm{ml}(95 \% \mathrm{Cl} 239,321)$. The difference between the groups was significant $(p=0.04)$. No correlation was found between $\mathrm{FEV}_{0.5}$ and markers of inflammation. Symptomatic children had lower lung function, regardless of the presence of infection $(p=0.004)$. Conclusion: Airway infection in infants with CF is associated with lower lung function but the presence of respiratory symptoms is more predictive of lower lung function than infection status. The primary defect in CF or other factors affecting airway calibre may lead to impairment of lung function and respiratory symptoms in the absence of infection.

G. Nixon is supported by the Grand Lodge of New Zealand Freemasons.

Key words: Cystic fibrosis, infant, lung function, infection, cough.
IRON DEFICIENCY IN CYSTIC FIBROSIS: RELATIONSHIP TO DISEASE SEVERITY AND PSEUDOMONAS AERUGINOSA INFECTION.

Reid DW, Withers NJ, Francis L, Wilsọn JW, Kotsimbos TC.

Cystic Fibrosis Service, Department of Respiratory Medicine and Monash University Medical School, Alfred Hospital, Melbourne, Victoria. Introduction; Iron deficiency (ID) is common in cystic fibrosis (CF) and is normally attributed to gastrointestinal factors and chronic inflammation socondary to sino-pulmonary sepsis. Hypothesis; Pseudomonas aeruginosa $(\mathrm{PA})$ requires iron for proliferation and we hypothesise that ID in CF might be directly related to PA infection and severity of lung disease. Methods; We measured iron status and several systemic markers of inflammation in $31 \mathrm{CF}$ patients (median age 27 years, range 21-36 years) and assessed the relationship to FEV1\%predicted, daily sputum volume and degree of pancreatic enzyme supplementation. Additionally, we measured the sputum concentrations of total iron and ferritin in an unselected subgroup of 13 subjects. Results; Seventy percent of subjects studied were ID (serum iron $\leq 7 \mu \mathrm{mol} / L$ \&/or transferrin\%sat. $\leq 16 \%$ ). Signiflcant relationships existed between serum iron, transferrin\%satn., C-reactive protein (CRP) \& ferritin:CRP ratio and FEVI\%pred. $(r=0.5, r=0.4, r=-0.7, r=0.7$ respectively, $p<0.05$ ). The volume of sputum expectorated daily was strongly associated with FEV1\%predicted, transferrin\%satn, and serum ferritin:CRP ratio $(p<0.05)$. No relationship existed between ID and pancreatic supplementation. Sputum concentrations of total iron (median $63 \mu \mathrm{mol} / \mathrm{L}$, range 17-134 $\mu \mathrm{mol} / \mathrm{L}$ ) and ferritin (median $5038 \mu \mathrm{g} / \mathrm{L}$, range $894-6982 \mu \mathrm{g} / \mathrm{L}$ ) exceeded normal plasma levels and negatively correlated with

FEV1\%predicted $(r=-0.6, r=-0.5$ respectively, $p<0.05$ ). Conclusions; ID in CF patients appears to be directly related to the severity of suppurative lung disease. Iron loss into the airway may facilitate PA infection and contribute to iron deficiency. 
TIME COURSE AND PREDICTIVE VALUE OF PERIPHERAL INFLAMMATORY MARKERS WITH INHALED BUDESONIDE TREATMENT Helen Reddel, Sandra Ware, Guy Marks, Cheryl Salome, Christine Jenkins and Ann Woolcock, Institute of Respiratory Medicine, Camperdown, NSW 2050

This study examined the effect of inhaled budesonide on blood eosinophils (EO), serum eosinophil cationic protein (ECP) and eosinophil protein $X$ (EPX). 61 subjects with poorly controlled asthma (60 atopic) received inhaled budesonide twice daily by Turbuhaler@. Budesonide dose was 3200 or $1600 \mu \mathrm{g} /$ day for 8 weeks, then $1600 \mu \mathrm{g} /$ day for 8 weeks, then budesonide dose was titrated over $\mathbf{5 6}$ weeks. Airway hyperresponsiveness (AHR) was assessed as $\mathrm{PD}_{20} \mathrm{FEV}_{1}$ histamine.

\begin{tabular}{|l|l|l|l|l|l|}
\hline Weeks & 0 & 8 & 16 & 48 & 72 \\
\hline $\mathrm{PD}_{20} \mathrm{FEV}_{1}$ histamine* $(\mu \mathrm{mol})$ & 0.15 & 0.90 & 1.22 & 2.66 & 3.19 \\
\hline Clinic FEV $_{1} \%$ pred, mean & 72.0 & 85.8 & 86.3 & 88.0 & 87.0 \\
\hline Eosinophils $(\mathrm{EO})^{*}\left(\mathrm{x} 10^{9} / \mathrm{L}\right) 0.26$ & 0.10 & 0.12 & 0.14 & 0.15 & \\
\hline Serum ECP* $(\mu \mathrm{g} / \mathrm{L})$ & 13.5 & 9.3 & 9.9 & 11.3 & 10.8 \\
\hline Serum EPX* $(\mu \mathrm{g} / \mathrm{L})$ & 44.3 & 25.0 & 27.4 & 27.4 & 27.6 \\
\hline
\end{tabular}

"geometric mean. Median final daily budesonide dose was $800 \mu \mathrm{g}$ for subjects completing 72 weeks $(n=42)$ and $1600 \mu \mathrm{g}$ for subjects withdrawn earlier ( $n=19$ ). EO, ECP and EPX levels were lowest at Week 8, but AHR continued to improve to Week 72. EO was lower at Week 8 for $3200 \mu \mathrm{g}$ cf. $1600 \mu \mathrm{g}$ starting dose $\left(0.07 \mathrm{cf} .0 .12 \times 10^{9} / \mathrm{L}, \mathrm{p}=0.02\right)$. There were positive correlations between baseline EO and both final $\mathrm{FEV}_{1} \%$ predicted $(r=0.45$, $p<0.001)$ and final $P_{20} \mathrm{FEV}_{1}(r=0.43, p<0.001)$. This study shows that maximal reduction in peripheral inflammatory markers occurs after 8 weeks budesonide. Higher baseline blood eosinophil count, but not serum ECP or EPX, appears to predict better outcome in lung function and airway hyperresponsiveness in subjects with poorly controlled asthma who receive inhaled budesonide treatment.

Supported by AstraZeneca Sweden, AstraZeneca Australia and NHMRC Key words: asthma, inflammation, eosinophils, ECP, EPX

Nominations for awards: Nil

USE OF INDUCED SPUTUM TO SELECT ADD-ON THERAPY IN PERSISTENT ASTHMA: A RANDOMISED TRIAL OF SALMETEROL IN NON-EOSINOPHILIC ASTHMA

Peter G Gibson, Jodie L Simpson and Nicholas Saltos Airways Research Centre, Department of Respiratory and Sleep Medicine, John Hunter Hospital, NSW, Australia

Both long-acting $\beta$-agonists and inhaled corticosteroids (ICS) are effective add-on therapy in persistent asthma. Clinicians usually make empiric choices between these 2 different classes of drugs. Induced sputum identifies a group with persistent asthma but suppressed inflammation (noneosinophilic) asthma in whom we hypothesised that Salmeterol (S) may provide better asthma control than ICS. 78 adults with stable symptomatic asthma were recruited and those with suppressed eosinophils $(E<5 \%, n=33)$ entered a 1 week run-in period followed by concealed random allocation to add-on therapy with fluticasone $500 \mu \mathrm{g} /$ day $(F)$ or $S 50 \mu \mathrm{g} /$ day with doubleblind evaluation of the treatment effect after 1 month. Baseline airway responsiveness (AR) was moderately increased, mean $P D_{20} 5.75 \mathrm{ml}$, and improved 2-fold to $11.5 \mathrm{ml}$ with $S(p<0.05)$, whereas $F$ led to no change in AR $(7.51 \mathrm{ml}, p>0.05)$. Sputum eosinophil cationic protein (ECP) was high at baseline $(2519 \mathrm{ng} / \mathrm{ml})$ and was not suppressed by therapy $(S=3673$.

$\mathrm{F}=2258$ ). Sputum $\mathrm{E}$ at baseline was $1.3 \%$, and remained unchanged after $\mathrm{S}$, but fell to $0.6 \%$ after $\mathrm{F}(\mathrm{p}<0.05)$. Peak flow improved by $28 \mathrm{~L} / \mathrm{min}$ with $\mathrm{S}$ $(p<0.01)$, but was not altered by $F(1.1 \mathrm{~L} / \mathrm{min})$. Daily symptoms and $\beta$-agonist use were reduced by $S(1.33$ putfs/day, $p<0.05)$, but not by $F$. Quality of life improved 0.8 units with $S(p<0.05)$ and 0.4 units with $F$. We conclude that in adults with persistent asthma, induced sputum can define a group with persistent AHR but suppressed eosinophilia. Salmeterol provides better asthma control than ICS in these patients. Induced sputum can help choose add-on therapy in persistent asthma.
EXHALED NITRIC OXIDE IS RELATED TO ALLERGIC SENSITISATION TO HOUSE DUST MITE AND CLADOSPORIUM

Sara H. Downs, Jörg D. Leuppi, Sue R. Downie, Guy B. Marks, Cheryl M. Salome Institute of Respiratory Medicine. University of Sydney, NSW, 2006

The concentration of nitric oxide in exhaled air (eNO) is associated with atopy and may also be a measure of airway inflammation. Individuals allergic to pollens and fungi have been reported to have more symptoms during seasons when the levels of the aeroallergens in ambient air are high. Aim: To determine if the association between eNO levels and sensitisation to specific allergens is the same in summer and winter. Methods: Skin prick test results for five allergens in 286 atopic children from Wagga and Moree were related to concentration of eNO in winter and summer 1998. At the skin prick test, a wheal size $>3 \mathrm{~mm}$ was defined as being sensitised. Expired air was collected in a $3 \mathrm{~L}$ wine cask bag and the NO content measured by chemiluminescent analyser Results: The proportion of children sensitised to house-dust mite was $64.3 \%$, to Alternaria was $43.4 \%$, to rye grass pollen was $53.5 \%$, to Cladosporium was $23.4 \%$ and to cat was $18.5 \%$. House dust mite and Cladosporium were significant predictors of eNO in both summer $(p<0.001)$ and winter $(p<0.001)$. Between winter and summer eNO increased in Moree $(p<0.001)$ and decreased in Wagga $(p<0.001)$. Sensitisation to house dust mite, Cladosporium, Alternaria, ryegrass or cat were not significant predictors of change in eNO in either town, although a negative association between Alternaria sensitisation and change in eNO in Wagga was of borderline significance $(p=0.03)$. Conclusion: In this sample of atopic children, eNO was related to sensitisation to house dust mite and Cladosporium. The direction of seasonal changes in eNO differed in the two towns, but were probably unrelated to allergic sensitisation. The factors which determine seasonal change in eNO remain unclear.

Supported by: Swiss Respiratory-, Scientific- and Novartis- Foundation, NHMRC, Lady Askin Trust, RPAH

Key words: Asthma, exhaled nitric oxide, skin prick test, allergen Nomination for Awards: none 
PERCEPTION OF ASTHMA: USEFULNESS OF A 10 YEAR PEF DIARY IN ASSESSING THE EFFECTS OF TREATMENT - A SINGLE CASE STUDY

K Bale, G Laszlo, AH Kendrick

Department of Respiratory Medicine. Bristol Royal Infirmary, Bristol, England.

Asthmatics can be classified as good or poor perceivers of airway function either in the laboratory or in the community. In a study done in $1988-90$ good perceivers were identified as having a significant negative correlation of visual analogue score (VAS) against coded PEF. Only ten of 300 patients were recording daily PEF using a Mini-Wright meter. We report a case of a good perceiver who has maintained a PEF and treatment diary card from first assessment in 1989 to re-assessment in 1999. Methods: The male patient, aged 66 in 1989, had known asthma. Perception of asthma was assessed in 1989 and 1999 using a $100 \mathrm{~mm}$ VAS with $0 \mathrm{~mm}$ being "No Asthman and $100 \mathrm{~mm}$ being "Worst Ever Asthma". PEF were recorded using turbine PEF meter up to 4 times daily for 14 days. The readings of PEF were not visible to the patient. The diary card, kept by the patient, showed twicedaily PEF. The variability of PEF and the effects of stated treatment for each day were analysed. Results: In 1989, PEF ranged from $30 \%$ to $107 \%$ predicted and VAS max $_{\text {was }} 93 \mathrm{~mm}$. VAS and PEF were significantly correlated $(r=-0.85)$, with a slope of $-1.03 \mathrm{~mm}^{-1}$. In 1999. PEF ranged from $87 \%$ to $120 \%$ predicted and VAS max $_{\text {mas }} 23 \mathrm{~mm}$. VAS and PEF were significantly correlated $(r=-0.33)$, with a slope of $-0.21 \mathrm{~mm} \%^{-1}$. Variation in PEF was up to $70 \%$ prior to March 1990 , with PEF ranging from 180 to $460 \mathrm{I}_{\mathrm{min}} \mathrm{m}^{-1}$, with inhaled $\beta_{2}$-agonists being the primary treatment. In March 1990 Becloforte improved PEF from a mean of $308 \pm 53 \mathrm{I}_{\mathrm{Imin}}^{-1}$ to $425 \pm 31 \mathrm{I} \cdot \mathrm{min}^{-1}$ Subsequently the variation in PEF was maintained to about $\pm 20 \%$, with $P E F$ ranging from 405 to $5201 . \mathrm{min}^{-1}$. Seven major reductions in PEF were observed ( $\triangle P E F>751 . \mathrm{min}^{-1}$ ), some requiring medical intervention.

Conclusion: The changes observed in perception demonstrate the probable effects of inhaled corticosteroids on perception of asthma, with a reduced slope compared to that in 1989. The control of asthma is clearly improved with marked reduction in PEF variability. The diary card has provided a unique insight into the variation of PEF and the requirements for treatment over 10 years in this patient.

Key words: Perception, Asthma

\section{AN OBSERVATIONAL STUDY OF MONTELUKAST IN ASTHMA}

Ian Charlton', Craig Mellis 2, Abe Rubinfeld 3, J Paul Seale 4

\section{(1) General Practice, Kincumber NSW 2251 (2) The New Children's} Hospital, Westmead NSW 2124 (3) Royal Melbourne Hospital, Parkville VIC 3052 (4) Department of Clinical Pharmacology. The University of Sydney NSW 2006.

Aim: To assess the effects of adding montelukast sodium (Singulair, MSD) to existing treatment in the control of asthma. Methods: Observational study. Patients were enrolled at the discretion of their physician, if their asthma was inadequately controlied on current treatment. Details of treatment and a symptom-based questionnaire were completed. FEV1 and PEF were recorded. Montelukast $10 \mathrm{mg}$ (5 mg in children) was added to current treatment. Patients were reviewed twice over a 12-week period. Results: Study population: $n=616$. The data for 132 completed patients have been analysed. Age range $2-91$ years; $77 \%$ aduits; $23 \%$ children; $62 \%$ female. $97 \%$ of patients had moderate to severe, frequent episodic or persistent asthma, and most were taking inhaled corticosteroids and long-acting B2agonists. Physicians reported that $69 \%$ of pafients had a moderate to substantial improvement while $29 \%$ had no improvement with montelukast. Post bronchodilator PEF increased from a mean of 331 to $362 \mathrm{~L} / \mathrm{min}$ in adults $(n=64)$. Improvement was reported in: symptoms in the past week, and, in the past 4 weeks, emergency visits to a hospital or a GP, days los from work or school, oral steroids, and overall patient satisfaction with preventer medication. 17\% (102/616) of patients discontinued treatment and $4 \%(23 / 616)$ experienced side-effects. Conclusion: $69 \%$ of patients with moderate to severe asthma demonstrated a sustained and clinically-relevant improvement in their asthma with the addition of montelukast to existing treatment for the 12-week period.

Supported by: A grant from Merck Sharp \& Dohme (Australia) Pty Limited Key words: Montelukast sodium, Singulair, severe asthma

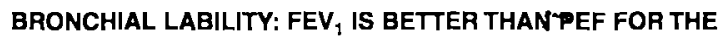
DIAGNOSIS OF ASTHMA.

David Belavy ${ }^{1}$, Charles Mitchell ${ }^{1} \&$ Brenton Eckert ${ }^{2}$

1Department of Medicine, The University of Queensland. 2Department of

Respiratory Medicine, Princess Alexandra Hospital, Brisbane, 4102.

Serial measurements of airway calibre are used in the diagnosis and management of asthma. Traditionally PEF is used to calculate Bronchial Lability $(B L)$ as: [highest daily PEF - lowest daily PEF]/highest daily PEF. FEV 1 is less effort dependent and more reliable than PEF, and has been shown to reflect flow in both large and small airways. Portable data storage spirometers now makes serial FEV 1 measurements possible. Aim: Determine the measurement of airway calibre (FEV 1 or PEF) and calculation of $\mathrm{BL}$ that best separates normal subjects from those with asthma. Method: On the basis of asthma questionnaire, spirometry and methacholine challenge, 25 well-defined asthma and 25 normal subjects were identified. All subjects then performed serial spirometry, recorded 7 times per day for 4-7 days using an electronic data storage spirometer (Micromedical DiaryCard, UK). Only sessions where spirometric data met ATS acceptability and reproducibility criteria were used in subsequent analysis. $B L$ indices of overall variability, within-day variability, between day variability and circadian variability for FEV 1 and PEF were calculated, and the upper limit of the normal range (95th percentile for normal subjects) determined for each index. Results: The traditional method of determining $B L$ using daily amplitude in PEF had an upper limit of normal of $18.2 \%$ and correctly identified only $50 \%$ of subjects with asthma. By contrast the coefficient of variation of $\mathrm{FEV}_{1}$ from all sessions $\left(\mathrm{CovFEV}_{1}\right)$ best separated the asthma

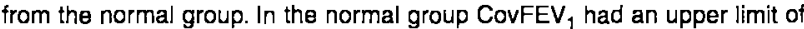
normal of $4.5 \%$. Values in excess of this level were strongly suggestive of asthma (sensitivity $=92 \%$ ). For all indices of $\mathrm{BL}, \mathrm{FEV}$, proved a significantly better measure than PEF $(p<0.01)$. Where the quality of spirometric data can be assured, FEV 1 proved more sensitive than PEF in separating the asthma and normal groups for all indices of $B L$. The index of BL providing the greatest sensitivity in the detection of asthma was CovFEV .

RECOVERY FROM AN ASTHMA EXACERBATION FOLLOWING A SINGLE HIGH DOSE OF INHALED CORTICOSTEROIDS - A PILOT STUDY. Sue R. Downie, Joerg D. Leuppi, Cheryl M. Salome, Ann J. Woolcock. Institute of Respiratory Medicine, University of Sydney. NSW. 2006.

The introduction of high dose inhaled corticosteroids (ICS) can lead to rapid improvements in lung function and asthma symptoms. A single dose of $2400 \mu \mathrm{g}$ of Budesonide can reduce airway responsiveness and sputum eosinophils within 6 hours. Aim: To determine if a single dose of $3200 \mu \mathrm{g}$ of Budesonide improves recovery from an asthma exacerbation. Methods: 19 Asthmatics with asthma exacerbation following withdrawal of ICS were randomised to receive either usual care (a doubling dose of ICS) plus placebo, or usual care plus a single dose of $3200 \mu \mathrm{g}$ budesonide, in a double-blind manner. Subjects monitored PEF, symptoms and rescue $\beta$-agonist use daily for 4 weeks. PEF was expressed as the lowest reading in each week as a $\%$ of the best reading in the four weeks pre exacerbation (PEF Low\%high). Symptom scores incorporated symptoms and $\beta$-agonist use, and had a range of $O$ to $B$. Ressults: The budesonide group had significantly lower symptom scores and higher PEF (Low\%high) in the first week after exacerbation than the placebo group. Both groups had recovered to similar levels by week 4 . (mean $\pm 95 \% \mathrm{Cl}$ ):

\begin{tabular}{|lllllll|}
\hline \multicolumn{3}{c}{ Mean Symptom Score } & \multicolumn{3}{c|}{ Mean PEF (Low\%high) } \\
Exac. & $2.2 \pm 0.9$ & $2.6 \pm 0.6$ & 0.53 & $84.9 \pm 3.3$ & $76.1 \pm 7.6$ & 0.08 \\
Wk 1 & $1.3 \pm 0.7$ & $2.5 \pm 0.5$ & 0.008 & $89.4 \pm 2.9$ & $81.7 \pm 3.6$ & 0.006 \\
Wk 4 & $0.2 \pm 0.2$ & $0.4 \pm 0.4$ & 0.4 & $90.3 \pm 3.49$ & $90.3 \pm 3.3$ & 0.99 \\
\hline
\end{tabular}

Conclusion: Asthma exacerbations treated by doubling the dose of ICS resolve more rapidly with the addition of a single high dose of $3200 \mu \mathrm{g}$ of budesonide. The effect is most marked during the first week after the exacerbation, when it is most likely to reduce morbidity due to the exacerbation. Supported by SNSF, Novartis-Found. NHMRC, Aust. ARDS-Assoc. Key words: Asthma, exacerbation, high dose ICS. Nominations for awards: Nil 


\section{P5}

A CLINICAL TRIAL OF AIR IONISERS IN THE TREATMENT OF ASTHMA Francesco Giorlando, Michael Abramson, Marc Cohen, Rosalie Woods Department of Epidemlology \& Preventive Medicine. Monash Medical School. Altred Hospital. VIC 3004

Air lonisers employ the generation of potential differences in the kilovolt range to produce negatively charged particles. The particles they emit are hydrated, mobile and highly reactive. Air ionlsation has been associated with altered serotonin metabolism in human and animal experiments. It is an effective means of precipitating airborne particles including particulate, airborne allergens and microorganisms, however, the effect of the use of these devices upon the clinical condition of asthmatics is the subject of controversy. This study aimed to assess the effect of nightly negative air ionisation (estimated 250000 negative ions/ml at 1 metre from emitters) upon measures of asthma in 53 adult subjects (28 active, 25 placebo). Methods: A double-blind, placebo-controlled, study design with two parallel groups was utilised. Following a 1 week run in, subjects used active ionisers or sham devices for 9 weeks. Lung function (pre and post bronchodilator $F E V_{1}, F V C, F E F_{25-75 \%}$ ) and Asthma Quality of Life were assessed in interviews at 5 week intervals. Subjects recorded peak flows, medication usage and symptoms twice-daily. Total plasma serotonin concentrations were atso measured. Results: There was a significant difference found between groups in the Social Disruption subscale. The active group showed less of an increase $(1.68, p=0.040)$ after sex adjusted analysis, suggesting a detrimental effect of air ionisation upon Quality of Life. Short acting bronchodilator usage increased more in the active group (1.82 doses per day, $p=0.19$ ) between weeks 5 and 6 than in the placebo group. Lung function scores were not significantly different between groups, although there was a trend for decreased FEV 1 and increased response to bronchodilator in the active group. Plasma 5-HT was increased in the active group by $101.5 \mathrm{nmol} / \mathrm{L}(\mathrm{p}=0.43)$ after 9 weeks of treatment. Conclusions: These results suggest that there may be a detrimental effect of the nightly use of air ionisers in asthma treatment. It is essential that further investigation of these effects be undertaken in well controlled patients to establish if the negative air ioniser usage is truly a risk factor for asthma exacerbations in the community.

Key words: asthma, air ionisation, clinical trial, serotonin

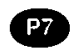

EFORMOTEROL: INFLUENCE OF DELIVERY DEVICE ON CLINICAL. EFFECT

McWilliams A M, Summers Q A.

Department of Respiratory Medicine, Royal Perth Hospital, Perth, W.A.

Longacting beta ${ }_{2}$ agonists now have an established role as an adjunct to Inhaled corticosteroids in asthma management. Eformoterol is available in two dry powder devices, Foradile Aerolizer and Oxis Turbuhaler(3). There has been no comparative study of their clinical effect. We compared the bronchodilator response, time to onset of response and time to maximum bronchodilator response following $12 \mu \mathrm{g}$ eformoterol delivered by these devices. Nineteen nonsmoking asthmatic patients with a documented bronchodilator response were enrolled in the randomised, single-blinded, crossover trial. The severity of baseline airflow limitation ranged from mild (FEV $1>70 \%$ predicted) to moderately severe (FEV $150-59 \%$ predicted). The patients were assessed on two separate study days at least one week apart. FEV was measured for a total of 6 hours after a single dosage of eformoterol. Side effects were also recorded. Statistical analyses were

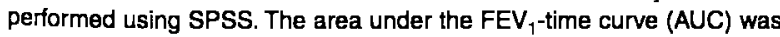
calculated for each device and evaluated by paired t-test. There was no significant difference in AUC, time to onset or time to peak effect. One third of patients experienced side effects with each device which resolved within the study period. These included tremor, nausea and headache. We conclude that there is no significant clinical difference between $12 \mu \mathrm{g}$ of eformoterol delivered by either device.

Key words: eformoterol, bronchodilator
ADDING FORMOTEROL IS MORE EFFECTIVE AND SAFER THAN DOUBLING THE DOSE OF INHALED STEROIDSIN MODERATELY SEVERE ASTHMA

Jenkins $C^{1}$, Mitchell $C$, Scicchitano $R$, Rubinfeld A. On behalf of 16 Australian Centres. ${ }^{1}$ Institute of Respiratory Medicine. Camperdown NSW 2050

Methods:In a prospective, multicentre, randomized, doubleblind, paraliel group study we compared the effectiveness of (A) adding formoterol (Foradilo(B))(FORM) dry powder inhalation $12 \mathrm{mcg}$ bd to beclomethasone (BDP) $500 \mathrm{mcg}$ bd with (B) doubling the BDP dose to $1000 \mathrm{mcg}$ bd in asthma patients on BDP 1000mcg a day or equivalent. Subjects: All had 2 of the following on 2 of the last 7 days of a 4 week run-in: daytime(DT) symptoms limiting activity; any nocturnal(NT) symptoms; use of at least 4 puffs of rescue bronchodilator(BD); diurnal variation in PEF of $>15 \%$. There were no differences between groups in baseline characteristics, symptoms, PEF and 2 hour post waking urinary cortisol/creatinine ratios (UCCR) as a measure of adrenal suppression. :The baseline values and changes at 12 weeks were:

\begin{tabular}{|c|c|c|c|c|c|c|}
\hline & \multicolumn{3}{|c|}{$A: F O B M+B D P 1000$} & \multicolumn{3}{|c|}{ B: $B D P 2000$} \\
\hline & L & Mean & Change* & $\underline{\mathrm{n}}$ & Mean & Change* \\
\hline MORNING PEF ( $(/ \mathrm{m})$ & 100 & 352 & +39 & 101 & 350 & +11 \\
\hline EVENING PEF $(/ / m)$ & 100 & 380 & +22 & 101 & 371 & +3 \\
\hline DT SYMPTOM SCORE & 100 & 1.42 & -0.93 & 101 & 1.44 & +0.45 \\
\hline NT SYMPTOM SCORE & 100 & 0.69 & -0.35 & 101 & 0.63 & -0.13 \\
\hline DT BD USE (puffs/day) & 100 & 3.20 & -2.27 & 101 & 3.32 & -0.89 \\
\hline UCCR (nmol/mmol) & 97 & 50.5 & +3.5 & 98 & 50.0 & -13.4 \\
\hline
\end{tabular}

Conclusion: FORM + BDP 1000 was more effective and resulted in less suppression of cortisol secretion than BDP 2000 in these symptomatic patients with moderately severe asthma

Supported by: Novartis Pharmaceuticals

Key words: asthma, formoterol, beclomethasone, adrenal suppression Nomlnatlons for awards: nil

"AS IF I HAVE NOT TAKEN A FULL BREATH" OR "HYPERVENTILATION SYNDROME" OR "SIGHING SYMPTOM" DUE TO GORD AND CURED WITH PHOPER TREATMENT.

Chitta M Mukerjee', Jane Carrick2 \& David Freiberg ${ }^{3}$

1SouthWest Specialist Medical Centre, Cabramatta, NSW. 2Dolphin Specialist Centre, Merrylands, NSW. ${ }^{3 B a n k s t o w n ~ R e s p i r a t o r y ~ C e n t r e, ~ B a n k s t o w n, ~ N S W . ~}$ Hyperventilation syndrome may be due to many causes, but gastrooesophageal reflux disease (GORD) has not been aetiologically linked to date. 21 cases have been identified. Three are reported here. Each was diagnosed with GQRD and each was cured with appropriate treatment. Case 1 (SE) A 25 year old Lebanese born female presented in May 1998 with a four year history of "as if I have not taken a full breath". She was sighing regularly. Lung function tests \& CXR were normal. Clinical examination was normal apart from an indented tongue and obesity. Gastroscopy showed one small area of reflux oesophagitis and biopsy showed H.pylori colonisation. She was treated with triple antibiotic therapy (Helicopack) for fourteen days. Her symptoms resolved and she remains symptom free after 18 months follow up.

Case 2 (JD) A 40-year-old male East Timorese refugee presented with a four-month history of "I can't breathe properly" especially when going upstairs. At night he woke with shortness of breath "as if 1 have not taken a full breath" and obtained some relief by drinking water. He also had a dry cough. Clinical examination revealed no abnormality except for repeated sighing. Lung function tests, CT scan and serum iron were normal. Gastroscopy showed a deep oesophageal ulcer (grade 3) at the lower end of the oesophagus. He was treated with Losec HP7 followed by Omeprazole 20 mgs bd for 6 months and 16 months later he was "very well".

Case 3 (SE) A 22-year-old Australian born male of Lebanese extraction presented in September 1996 complaining of "as if I have not taken a full breath" and sighing many times each day for 4-5 years. His wife feared that "my husband does not like me". Spirometry and CXR were normal. Gastroscopy showed severe ulcerative oesophagitis (grade 4). He was treated with omeprazole $20 \mathrm{mgs}$ bd for 6 months and at last follow up on 9/11/99 he said, "I am very well thank you. You cured me long ago". GORD can cause the above mentioned symptom which is curable and physicians should be aware of it and treat accordingly. 


\section{P9}

SHOULD INHALED CORTICOSTEROID TREATMENT "START HIGH" IN POORLY CONTROLLED ASTHMA?

Helen Reddel, Christine Jenkins, Guy Marks, Sandra Ware, Wei Xuan, Cheryl Salome, CaroAnne Badcock*, Ann Woolcock. Institute of Respiratory Medicine, Camperdown, NSW 2050: *AstraZeneca, Ryde, NSW 2113

Objectives: To determine if short-term or long-term outcomes in subjects with poorly controlled asthma can be improved with a starting dose of inhaled budesonide higher than that recommended in international guidelines. Methods: Parallel-group study of 61 subjects with poorly controlled asthma, randomised to receive $3200 \mu \mathrm{g}$ or $1600 \mu \mathrm{g}$ budesonide daily by Turbuhaler@ for 8 wks (double-blind), then $1600 \mu \mathrm{g} /$ day for 8 wks (single-blind), followed by 14 months of open-label budesonide dose titration using a simple clinical algorithm. Study design incorporated a written asthma crisis plan based on electronic PEF monitoring. Primary outcome variable for Wks 1-16 was change in airway hyperresponsiveness (AHR), and for the open-label phase, mean daily budesonide dose. Results: During Wks 1-16, subjects starting with $3200 \mu \mathrm{g} /$ day were 3.8 times more likely to achieve remission of AHR ( $\left.\mathrm{PD}_{20} \mathrm{FEV}_{1} \geq 3.92 \mu \mathrm{mol}\right)$ than those starting with $1600 \mu \mathrm{g} / \mathrm{day}$, but other outcome measures did not differ significantly (e.g. increase in morning peak expiratory flow $+134 \mathrm{cf} .+127 \mathrm{~L} / \mathrm{min}, \mathrm{p}=0.8$ ). Subjects achieving remission were less likely to experience an exacerbation (2/13 cf. 27/48, $p=0.02$ ). During budesonide dose reduction, there was no significant difference in mean budesonide dose ( $1327 \mu \mathrm{g} /$ day cf. $1325 \mu \mathrm{g} /$ day, p $>0.3)$. By completion/withdrawal, there was no significant difference in overall improvement in AHR ( $+3.9 \mathrm{cf}$. +4.2 doubling doses, $p>0.6$ ) or in asthma control (median symptoms $0.0 \mathrm{cf}$. 0.0 days/wk, $\beta_{2}$-agonist use $0.0 \mathrm{cf} .0 .1$ occasions/day, $p>0.8$ ). Median compliance with electronic monitoring was $89 \%$ over the whole study. Concluslons: A high starting dose of budesonide in subjects with poorly controlled asthma caused more rapid remission of AHR, but tight control of asthma was achieved with both starting doses.

Supported by AstraZeneca Sweden, AstraZeneca Australia, and NHMRC Key words: asthma, inhaled corticosteroids, airway hyperresponsiveness

THE WORDS TO DESCRIBE METHACHOLINE INDUCED AIRWAY NARROWING IN ASTHMATICS AND NON-ASTHMATICS

Alyson Roberts and Cheryl Salome

Institute of Respiratory Medicine. University of Sydney NSW 2006

Symptoms of wheeze or chest tightness, reported in questionnaires, form part of the definition of asthma in epidemiological studies, but these words may have different subjective meanings. Aim: To determine if the appropriateness of the terms used to describe methacholine induced airway narrowing differs between asthmatics and non-asthmatics, or, in a community sample, between subjects with reported wheeze and those who have not wheezed. Methods: 23 non-asthmatic and 19 asthmatic subjects underwent methacholine challenge $(0.04-200 \mu$ moles $)$, to cause respiratory discomfort. They then marked a visual analogue scale (VAS) to rate the appropriateness of each of 9 terms to best describe the sensations induced by the methacholine. The distance of the mark from the left-hand end of the VAS labelled 'not at all appropriate' was recorded as the \% of the total VAS length. This protocol was repeated in subjects selected from a population sample, with $(n=32)$ and without $(n=20)$ reported recent wheeze. Results: The asthmatics $(23.5 \pm 6.3 \%)$ had a slightly greater \% fall in FEV 1 than the non-asthmatic subjects $(16.2 \pm 4.6 \%)(p=0.06)$. Asthmatics rated '" feel wheezy' $(68.2 \pm 13.1 \%)(p<<0.001)$, 'My chest feels tight' $(68.9 \pm$ $13.8 \%)(p<<0.001)$ and 'I find it hard to breathe' $(61.4 \pm 13.0 \%)(p<<0.001)$ as significantly more appropriate descriptors than did non-asthmatics. In the population sample, subjects with wheeze in the last 12 months rated 'I feel wheezy' $(47.6 \pm 11.6 \%)$ as significantly more appropriate than did nonwhe hyperresponsiveness. Conclusions: Asthmatics and non-asthmatics differ in the words used to describe sensations associated with airway narrowing. It is not known if this difference reflects different physiological changes in the airways. Subjects who report recent wheeze, consider 'l feel wheezy' to be a more appropriate term to describe induced bronchoconstriction than do subjects with no history of wheeze. This may have implications for the interpretation of symptoms recorded by questionnaire in epidemiology. Supported by the NHMRC

Key words: asthma, perception, symptoms, epidemiology
PERSONAL BEST AND RECENT BEST PEAK FLOW IN ASTHMA MANAGEMENT - IMPLICATIONS FOR ASTHMA ACTION PLANS Helen Reddel, Guy Marks, Cheryl Salome, Christine Jenkins, Sandra Ware, Ann Woolcock, Institute of Respiratory Medicine. Camperdown. NSW 2050

Many PEF-based asthma action plans suggest a change in treatment when morning PEF falls below a trigger point calculated from a reference value such as personal best PEF or predicted PEF. Recent guidelines suggest once-daily PEF monitoring during good asthma control. Aim: To examine PEF reference values and simple PEF indices during inhaled corticosteroid treatment, using morning only and twice-daily values. Methods: 61 subjects with initially poorly controlled asthma recorded PEF electronically twice daily during 72 weeks of inhaled budesonide treatment. For each week, the lowest morning PEF (Low) was identified, together with highest PEF (High=recent best) and personal best PEF ( $P B=$ cumulative best PEF). PEF indices (Low\%High, Low\%PB, and Low\%predicted) were examined at baseline (poor control) and at plateau (good control). Results:

\begin{tabular}{|l|c|c|l|}
\hline $\begin{array}{l}\text { PEF index } \\
\text { median (IQR) }\end{array}$ & Poor control & Good control & $\begin{array}{l}\text { Level at good control, } \\
\text { cf. Low\%PB }\end{array}$ \\
\hline Low\%PB & $63 \%(48,73)$ & $81 \%(76,84)$ & - \\
\hline Low\%High & $63 \%(48,73)$ & $89 \%(85,92)$ & $p<0.0001$ \\
\hline Low\%predicted & $49 \%(40,66)$ & $82 \%(72,95)$ & $\mathrm{p}=0.02$ \\
\hline
\end{tabular}

During poor control, Low\%High and Low\%PB were substantially higher $(+7$ percentage points) when based only on morning PEF compared with twice daily PEF; during good control the differences were still significant ( $p<0.0001)$ but small ( +2 percentage points). Conclusions: This study shows that recent best, personal best and predicted PEF are not interchangeable as reference values in asthma action plans; recent best (High) is preferred as it does not need continuous PEF monitoring. A trigger point of $<80 \%$ personal best in action plans would result in overtreatment. During good asthma control, indices of PEF variation are only slightly overestimated by recording PEF once daily in the morning.

Supported by AstraZeneca Swoden, AstraZeneca Australia and NHMRC Key words: asthma management, asthma crisis plans, PEF monitoring Nominations for awards: $\mathrm{Ni}$

\section{* Refer to page A18 for P10A}

NEBULISER AND PRESSURE OUTPUT FROM THE MEFARTM DOSIMETER: RESULTS FROM ECRHS CALIBRATION PROJECT. R.J Ward, D.P. Johns, B. Skoric, E.H Walters. Department of Respiratory Medicine. The Alfred Hospital and Monash University Medical School, Melbourne, Australia.

The European Respiratory Health Survey (ECRHS) is a large multicentre longitudinal survey investigating the prevalence of asthma around the world. Mefar ${ }^{T M}$ nebulisers and dosimeters are currently used in the ECRHS, to determine the level of airway responsiveness. We have demonstrated in the past that differences exist in the driving pressure developed by different MefarTM dosimeters, this significantly changes aerosol output. Aerosol output can also significantly differ between batches of the same model of nebuliser. Due to these concerns over the accuracy of the Mefar ${ }^{T M}$ system used in phase 1 ECRHS and its potential confounding effect on the results, we were commissioned by the ECRHS Coordinating Committee to calibrate all the nebulisers to be used in the second phase of the ECRHS (phase II). We measured the mean aerosol output from 366 new Mefar ${ }^{\top M}$ nebulisers manufactured in 2 batches. We also studied the effect of changing dosimeter driving pressure on aerosol output in the nebulisers $(n=5)$. Nebuliser output was measured using a locally developed calibration method using lithium chloride. Nebuliser output was significantly different between the two batches $(p<0.005)$. The mean $\pm(S D)$ aerosol output for batch 1 was 7.0 mg. $\sec ^{-1}(0.8)$ and for Batch 2 mean $6.3 \mathrm{mg} \cdot \mathrm{sec}^{-1}(0.7)$. The combined mean of all 366 nebulisers was 6.4 mg.sec $^{-1}(0.8)$ range $3.1-8.4$ mg.sec $^{-1}$. The mean aerosol output of 5 nebulisers studied was directly related $\left(\mathrm{R}^{2}=0.99\right)$ to dosimeter driving pressure with range of $5.1(0.7)-9.0(1.3)$ mg.sec-1 between $150-250 \mathrm{kPa}$. These findings are important because we have shown that significant differences in aerosol output can occur at different driving pressures. These potential confounders could compromise phase II of the ECRHS. Therefore these differences in aerosol output should be taken into account during statistical interpretation of the final data. 
THE COST-EFFECTIVENESS OF INHALED FLUTICASONE PROPIONATE AND BUDESONIDE IN AUSTRALIAN ADULTS AND CHILDREN WITH ASTHMA

John Wilson', Carolyn Rutherford?

1) Department of Respiratery Medicine. Alfred Hospital, Prahran, 3181 and (2) Glaxo Wellcome Australia Ltd, Boronia, 3155

A meta-analysis has compared the efficacy of two commonly used inhaled corticosteroids, fluticasone propionate (FP) and budesonide (BUD), at the clinically equivalent dosing ratio of $1: 2$ in 1980 patients (1). All clinical efficacy measures showed greater improvements with FP than BUD. Limited data are available on the relative economic benefit of FP and BUD, particularly in Australia, hence an economic analysis has been conducted based on the meta-analysis. Methods: The economic analysis was conducted from the perspective of the Australian healthcare system. Costs to the healthcare system (eg. drugs, medical consultations, hospitalisations) were calculated by applying Australian unit costs to health care resource use recorded during the studies. Sensitivity analyses were conducted. Results:

\begin{tabular}{|l|l|l|l|}
\hline Endpoint & FP & BUD & P value \\
\hline Total cost per patient per week & $\$ 10.96$ & $\$ 18.77$ & \\
\hline Successfully treated week & $42 \%$ & $34 \%$ & $<0.001$ \\
\hline Cost per successfully treated week & $\$ 26.28$ & $\$ 54.88$ & \\
\hline Symptom-free days & $45 \%$ & $42 \%$ & 0.036 \\
\hline Cost per symptom-free day & $\$ 3.46$ & $\$ 6.41$ & \\
\hline Episode free days & $31 \%$ & $27 \%$ & 0.003 \\
\hline Cost per episode-free day & $\$ 5.05$ & $\$ 10.04$ & \\
\hline
\end{tabular}

-improvement from baseline in Peak Expiratory Fow (PEF) of $5 \%$ or more of PEF predicted -.days on which patients had no symptoms, no use of rescue medication, no disturbance in sleep pattern and an absence of adverse effects

Conclusion: Treatment with FP costs less than BUD at a dosing ratio of 2:1. In addition, FP showed greater improvement in PEF and was therefore more cost-effective than BUD,

Reference (1) Barnes NC, Hallett C, Harris TAJ. Respir Med 1998;92:95-104. Key words: asthma, fluticasone, budesonide, cost-effectiveness

\section{P15}

EVALUATING ASTHMA CARE IN AUSTRALIAN AND BRITISH GENERAL PRACTICES lan Chariton

Aims. To conduct a case control study comparing a nurse run asthma clinic with traditional Australian general practice asthma care and compare these results to a nurse run asthma clinic in the UK.

Methods. A case control study was conducted comparing patients attending a nurse run asthma clinic in Kincumber N.S.W. with control patients in neighbouring surgeries at Saratoga and Avoca. Eighty three patients who attended the asthma clinic were matched for age, sex and disease severity. Patient case notes were audited to determine the number of consultations with the doctor, courses of oral steroid and use of acute salbutamol nebulisations. These results were compared to a nurse run asthma clinic in Aylsham Norfolk. Intervention. The nurse run asthma clinics in Australia and the UK were run in similar ways. Patients using inhaled steroids were invited by their GP to a clinic conducted by an asthma nurse. The nurse spent one hour explaining the mechanism, treatments, and seif management of asthma. This was reinforced with a peak flow meter and diary card. Patients were reviewed by the nurse at 12 weekly intervals. Patients could consult their own doctors at any time if they wished.

Results Practices and patients were similar in the study groups. Australian practices had a higher proportion of children compared to the UK.(54\% v $30 \%)$. GP consultation times were longer in Australian practices(15 v 10 minutes). In the UK clinic 1.7 courses of oral steroids were prescribed in the 6 months before the clinic versus 0.5 after $(p<0.001)$. Australian clinic: 0.4 beiore 0.3 after $(p=N S)$. Australian control 0.4 before 0.3 after $(p=N S)$.

The percentage of patients who would like their doctors to talk more about their asthma was $70 \%$ in the UK patient group and $22 \%$ for the Australian control practice and $13 \%$ for Australian asthma clinic practice. The difference between Australian and UK groups was significantly different $(p<0.05)$

Conclusions The Australian asthma clinic failed to demonstrate appreciable advantages over well managed GP care. Patients attending the UK asthma clinic had greater morbidity than in Australia. After attending the UK asthma clinic the patients' asthma improved to a level comparable to the Australian asthma patients. The differences between the UK and Australia may be due to the progress general practice has made in the 5 years between the studies or the greater amount of time Australian GPs spend with their patients. Asthma clinics may be most appropriate where patient care is less than ideal or where doctors themselves do not feel confident in managing asthma at the level current guidelines recommend.

\section{A CONTROLLED STUDY TO ASSESS THE EFFECTIVENESS OF A GENERAL PRACTITIONER AND ASTHMA NURSE EDUCATION SESSION WITH AUSTRALIAN PRIMARY SCHOOL TEACHERS. lan Charlton}

Objective. To evaluate the effectiveness of a one hour education programme conducted by a local GP and Asthma Nurse with a group of primary school staff.

Methods. Four schools from the Central Coast of N.S.W. were randomly selected. Intervention schools were matched with schools of a similar size and location and whether they had received asthma education in the past. Questionnaires evaluating knowledge, attitudes and response to scenarios were administered before and after to intervention and control schools.

Intervention schools received a one hour tutorial outlining the basic mechanisms of asthma, use of inhalers and spacers and treatment of emergency episodes was undertaken. They also received an asthma first aid kit, wall posters and reading material. Control schools received no contact other than requests to complete the before and after questionnaires.

Results Seventy two teachers participated in the intervention and 60 teachers provided information for the control group. Knowledge scores prior to the intervention were high. The knowledge of teachers after the intervention was generally better than that of the teachers in the control schools, although statistical significance was only recorded for the teacher's awareness of the schools asthma policy $(p<0.03)$.

A higher proportion of teachers in the intervention group were able to provide a suitable response to the scenario questions after the intervention compared to the control group ( $p<0.07$ ). After the intervention, $68 \%$ of the teachers were still unable to identify the appropriate steps to take when salbutamol therapy failed to be effective.

Conclusions. This study outlines the difficulty of developing programmes to improve teacher's management of asthma in the school situation.

Programmes that involve a more active participation in the learning process may be more effective rather than the seminar approach used in this study. HEALTH SERVICE UTILISATION AND ACTIVITY LIMITATION IN
ASTHMATICS FROM A SOUTH AUSTRALIAN POPULATION

DJ Christopher, B Smith, D Wilson, AM Southcott B Ruffin, Dept of Respiratory Medicine, University of Adelaide, TQEH Campus, SA- 5011

This study looks at the utilisation of the health services by the asthmatics in the community and the limitation to physical activity and work caused by the disease. We performed a survey of a representative adult population of 3010 South Australians. Yn these, face-to-face interviews recorded: demographic data, details of past medical diagnosis of asthma $\&$ other chronic lung diseases; asthma symptoms; medical services available to asthmatics and activity limitation on account of the disease. Quality of life was measured using standard quality of life measures.

Results: In 299 patients $(\mathbf{9} .9 \%$ ) a diagnosis of asthma had been made by a doctor. Over a 12 month period, in comparison to the rest of the study population, a significantly higher number of asthmatics utilised the services of the general practitioner ( $59 \%$ VS $43.9 \%$; $P<001)$, the hospital (12\% VS $7.7 \% ; P<0.01$ ) and the other health services ( $34.1 \%$ VS $24 \% ; P<0.001)$ and were unable to obtain health services that they needed $(12.4 \%$ VS $7.9 \%$; $P<0.007)$. Significantly more asthmatics were using medications for a chronic disease for more than 6 months in the previous 12 months, when compared to the rest of the population ( $50.5 \%$ VS $22.6 \% ; P<0.001)$. A higher number of asthmatics were unable to carry out their normal duties on account of their disease (20.9\% VS $14.7 \% ; \mathrm{P}<0.004)$ among those who were able to carry out normal activities, more asthmatics tended to cut down the activities or accomplish less $(35.6 \%$ VS $19.2 \% ; P<0.001)$. The quality of life scores were lower for asthmatics when compared to the rest of the population.

Conclusion: Asthmatics in the community seem to have the need to utilise the health services more and the disease seems to impose significant limitation to activity and work.

Key words: asthma, health services, work limitation, quality of life "This research was funded by The Queen Elizabeth hospital Research Foundation. 
QUALITY OF LIFE IN PERENIIAL ALLERGIC RHINITICS IN SYDNEY Sue R. Downie, Morgan Andersson, Joerg D. Leuppi, Cheryl M. Salome, Janet Rimmer. Institute of Respiratory Medicine. University of Sydney, NSW. 2006.

House dust mite (HDM) allergen levels in Sydney are high by national and international standards. Although allergic sensitisation to HDM and rhinitis are common in Sydney, little is known about the impact of perennial allergic rhinitis on quality of life (QOL) in a high allergen environment. Aim: To compare QOL measures in HDM sensitised, ininitic subjects and healthy non-atopic controls, over a period of one year. Methods: 40 rhinitis patients skin prick test positive to D.farinae or D.pteronyssinus, and 19 nonatopic controls were enrolled in a longitudinal one year study that involved $\mathrm{QOL}$ assessment at 3 monthly intervals. The disease-specific $\mathrm{QOL}$ questionnaire (Juniper, 1997) measures the effect of rhinitis on daily activity, sleep, non-nasal non-eye problems, practical problems, nasal and eye symptoms and emotions. Results: In all measures, the QOL was significantly worse in thinitis patients than controls both in summer and winter. There were no significant differences between summer or winter. Compared to healthy controls, rhinitis subjects were not only significantly more troubled by nose $(p<0.0001)$ and eye $(p<0.001)$ symptoms, they were also more troubled by non-nasal non-eye problems $(p<0.01)$ such as fatigue, poor concentration, headache and reduced productivity. In rhinitics who were sensitised to pollens and HDM $(n=19)$, QOL measures did not differ significantly from those with HDM sensitisation only, and they did not have significant seasonal changes in quality of life. Conclusions: In a high allergen environment, perennial minitis has a significant impact on quality of life, and affects not only specific symptoms, but also non-specific measures of quality of life such as fatigue, poor concentration, headache and reduced productivity. Seasonal allergens appear to have little additional impact in the presence of high levels of perennial allergens.

Supported by Astra, Lund.

Key words: Perennial rinitis, quality of life, house dust mite, allergens Nominations for awards: Nil

\section{P19}

ADULT ASTHMA RISK SCREENING QUESTIONNAIRE INVOLVING SEVERITY PREDICTS QUALITY OF LIFE

Pilotto LS1, Smith BJ2, Heard A3 Bennett PW4, Weekley J1, Nobes E1, DeLucia $A^{4}$, Ruffin RE2, McElroy $\mathrm{HJ}^{1}$.

(1) Clinical Epidemiology and Health Outcomes Unit. North West Adelaide Health Service, Adelaide 5011, (2) Department of Medicine. University of Adelaide. (3) Department of Human Services, Adelaide and (4) Adelaide Western Division of General Practice. Adelaide 5011.

An adult risk screening questionnaire (RSQ)1, principally involving severity, has previously been shown at a score above 30 to predict attendance at hospital emergency departments. We are using a modified form of this questionnaire in a RCT of asthma clinics in general practice. From baseline data, we have used the RSQ to assess asthma severity as a predictor of quality of life (QoL). Methods: 12 general practices in Adelaide. The RSQ covered sleep disturbance, hospital admissions, number of GPs visited and use of oral steroids for asthma, and a patient's rating of the severity of their asthma. The St. George Respiratory questionnaire (StGQ) was used to measure QoL. Results: 167 adult asthmatics were recruited, with a mean (sd) age of 50.4 years ( \pm 16.7 years). 56 participants scored over 30 on the RSQ. Age, weight, number of co-morbidities, history of smoking, $\%$ predicted $\mathrm{FEV}_{1}$ and RSQ were independently related to the StGQ score. A stepwise regression of these variables showed a RSQ score $>30$ to be a good predictor of the StGQ score after adjusting for number of comorbidities and history of smoking $(p<0.001)$. On average, people with a RSQ score > 30 will have a StGQ score 13.4 points higher (95\%Cl: 8.7 , 18.2) than those with score $\leq 30$. This is a clinically significant difference. Conclusion: The RSQ, used as a measure of severity, is a good predictor of clinically significant differences in QoL in adult asthmatics, and is sufficiently brief to be useful in general practice, and aiso as an alternative to lengthy QoL measures in some research settings.

'Wakefield $M$, Ruffin $F$, et al. A risk screening questionnaire for adult asthmatics to predict attendance at hospital emergency departments. Chest 1997; 112: 1527-1533. Supported by Commonwealth Department of Health and Aged Care Key words: Asthma, severity, quality of life
DEVELOPMENT OF A QUESTIONNAIRE FOR ESTIMATING ADHERENCE TO ASTHMA MEDICATION

Rod O'Connor'1, Peter Gibson², Christine Jenkins 3 , Charles Mitcheil/4 \& Matthew Peters 5

(1) School of Health Services Management, University of New South Wales. 2052 (2) Department of Respiratory Medicine. John Hunter Hospital, 2305 (3) Institute of Respiratory Medicine, Royal Prince Alfred Hospital, 2050 (4) Department of Respiratory Medicine. Princess Alexandra Hospital, 4102 (5) Bespiratory Department. Concord Repatriation General Hospital, 2139

The AMAQ (Asthma Medication Adherence Questionnaire) has been developed to estimate adherence to asthma preventer medication based on patients' attitudes and behaviours. Methods: Literature review and patient interviews were used to identify items that could measure traits and behaviours relevant to adherence, along with questions to record reported adherence. Items were trialled to assess appropriateness, clarity, and comprehensiveness, modified through several draft versions, and then presented to patients by a non-clinician, emphasising that non-adherence was known to be common, honesty was desired, and responses were confidential. Results were analysed using Factor Analysis to refine item clusters representing each domain (eg. perceived stigma, asthma severity), and Cronbach Alpha was used to reduce the number of items in each cluster and enhance reliability. Multiple regression was then applied to select and weight items to form a scoring method predictive of nonadherence. Results: Analyses were based on 26 subjects under 50 years of age (all patients over age 50 reported full adherence). Seven item clusters were selected to be predictive of non-adherence, eg. 'perceived stigma', 'subjective estimate of asthma seriousness', 'locus of control'. The model predicted preventer non-adherence with multiple $\mathrm{R}=.90 ; \mathrm{R}^{2}=.81$; Adjusted $\mathrm{R}^{2}=.69, \mathrm{~F}(8,13)=6.88, p<.001$. Conclusions: The $A M A Q$ offers a survey instrument that estimates non-adherence based on reported attitudes to asthma medication. It also provides a systematic and comprehensive assessment of attitude factors and behaviours associated with nonadherence, and could be used to allocate subjects a priori to treatment groups in clinical trials to control for non-adherence effects. Key words: asthma, medication adherence, questionnaire Supported by Glaxo Wellcome Australia

\section{SMOKING IN ADOLESCENTS WTH ASTHMA: PREMEDICATION TO PROMOTE SOCIAL PARTICIPATION}

S Sawyer1, R Aroni², D Goeman², M Abramson³, F Thien ${ }^{4}$, J Douglass ${ }^{4}$, K Stewart

Centre for Adolescent Health ${ }^{1}$ LaTrobe University ${ }^{2}$, Monash University ${ }^{3}$. Alfred Hospitals and College of Pharmacy 5

There is little understanding about what most influences decision-making around smoking in young people with asthma. We aimed to examine the perceptions, attitudes and behaviours of adolescents with asthma, focussing on understanding the experiences and practices of smoking and its impact on asthma and medication use. Method: 90 young people ( $10-24$ years) were recruited from 3 tertiary asthma clinics and participated in an in-depth interview that was recorded and transcribed. Content and thematic analyses were undertaken, with data management assisted by NUD*IST. Results: 57 participants reported to be non-smokers, 11 classified themselves as smokers, 4 as former smokers and 7 as marijuana smokers. Smoking status was not detailed in 11. Across all age groups participants knew that smoking was bad for their health. However, nearly all smokers in the sample mentioned initial peer pressure, via constructions of "being cool", as contributing to their smoking. This was frequently despite acknowledging that smoking made their asthma worse. The smokers chose to premedicate with reliever medications prior to social events where they knew that they and/or others would be smoking. This behaviour was paralleled among the non-smokers who premedicated prior to participating in social events where they expected to be exposed to passive smoke. Both groups viewed this as a reasonable means of participating in social activities without overly compromising short term health outcomes. These findings indicate that smoking (passive and active) is commonly experienced as an asthma trigger, but that in smoking adolescents, this knowledge is more influential in informing use of reliever medication than in stopping smoking. This suggests that smoking cessation strategies must be attuned to both social as well as health contexts.

Supported by the NHMRC and Asthma Victoria (Anna Jane Trust) Key words: Asthma, adolescents, smoking, premedication Nominations for awards: Nil 
ADHERENCE IN ADOLESCENTS WITH ASTHMA: COMPARISON OF SELF REPORT WITH PARENTAL REPORT AND OBJECTIVE MEASUREMENT

\author{
SM Sawyer', V Dakin
}

Centre for Adolescent Health and Department of Respiratory Medicine ${ }^{1}$ Boyal Children's Hospital, Victoria, 3052

The extent of poor adherence with preventative medications for asthma is increasingly recognised. Adolescents are widely believed to be less adherent than other populations although there is little evidence to support this belief. The reliabillty of adolescent self report (AR) versus parent report (PR) of adolescent medication taking is unknown. We aimed to measure adherence with preventative asthma medications in adolescents and compare AR and PR of adherence with objective measures of adherence. Method: 30 consecutive adolescents were enrolled following routine review at a tertiary adolescent asthma clinic. An electronic Doser was attached to all metered-dose inhalers (MDI) for one month. Telephone follow up with adolescents and parents (separately) identified AR and PR for the last 7 days and the total study period, using clinical approaches to optimise self report. Results: Mean adherence rates for preventative medication were $71 \%$ (last 7 days) and $78 \%$ (study period). However, adolescents were fully adherent on only $40.6 \%$ (last 7 days) and $53.4 \%$ (study period) of days. Extra dosing was identified throughout the study period, rather than rapid MDI emptying or 'dumping'. There was moderate association but weak agreement with AR over the last 7 days of monitoring only (ICC $=0.25)$. PR showed poor agreement with objective monitoring over both time periods. PR agreed better with AR. Concluslons: Mean adherence in this selected population of adolescents is as high as has been previously reported in any population with asthma. However, adolescents were fully adherent on less than half of all days studied. Adolescents reported medication use more reliably than parents over the last 7 days of monitoring.

Supported by Asthma Victoria (Helen Schutt grant) Key words: Asthma, adolescents, adherence, electronic monitoring, self report. Nominatlons for Awards: Nil

TEACHING ASTHMA MANAGEMENT IN FINAL YEAR MEDICAL. STUDENTS: AN EVIDENCE-BASED APPROACH

D H Yates', S Shah', E Veitch', PS Thomas ${ }^{3}$. Depts of Respiratory Medicine, Concord ${ }^{1}$ and Prince of Wales Hospitals ${ }^{3}$ and Dept of Community Medicine, Auburn Hospital3, NSW

Asthma management plans and asthma education are incorporated into guidelines, yet teaching about asthma in medical school is seldom assessed. We studied asthma knowledge, confidence in asthma management, and effectiveness of an asthma education workshop. Methods: Interactive teaching sessions on management of asthma and a practical session using asthma devices. Theoretical knowledge was assessed by a 20 item questionnaire, completed before the workshop at 2 wks. Practical knowledge was assessed by confidence scores in use of asthma devices (0-5 : not confident - very confident) before, immediately after the workshop, and at 2 weeks. Results: Mean (SE) knowledge scores increased significantly from $64.4(6.1) \%$ to $74.2(5.6) \%$ ( $p<0.05$, paired t-test) at 2 weeks. Students scored poorly in questions on: predictors of asthma mortality, nebuliser and Turbohale ${ }^{R}$ use, asthma management plans, side effects of inhaled glucocorticosteroids, and physical signs in acute asthma. Confidence scores rose significantly for all practical aspects of asthma management ( $p<0.01$; Wilcoxon matched pairs test), and declined at 2 weeks, but still remained significantly higher than baseline. Mean (SE) scores:

$\begin{array}{llll} & \text { Pre } & \text { Post } & 2 \text { weeks } \\ \text { Discussing asthma } & 3.3(0.8) & 4.4(0.6)^{* *} & 4.1(0.7)^{* *} \\ \text { Use of PF meter } & 4.0(0.9) & 4.6(0.6)^{* *} & 4.4(0.7)^{*} \\ \text { Use of MDI } & 3.8(0.2) & 4.7(0.5)^{* *} & 4.3(0.7)^{*} \\ \text { Use of spacer } & 3.5(0.9) & 4.7(0.5)^{* *} & 4.3(0.6)^{*} \\ \text { Use of turbohaler } & 3.3(0.9) & 4.7(0.5)^{* *} & 4.1(0.9)^{*} \\ \text { Use of nebuliser } & 3.1(1) & 4.6(0.8)^{* *} & 4.0(0.8)^{* *}\end{array}$

Conclusions: Medical student knowledge about several important features of asthma care was poor. Our workshop increased knowledge and contidence in management of asthma.

\section{PEER-LED ASTHMA EDUCATION IMPROVES QUALITY OF LIFE IN ADOLESCENTS}

S Shah, J Peat, G Cantwell. $H$ Wang. P Sindusake. R Henry, P Gibson Asthma is a major health problem in adolescents. The Adolescent Asthma Action (Triple A) Program is the first peer-led asthma education program, which empowers young people to take control and improves knowledge and attitudes about asthma in the school community.

Aim: To establish the effect of the Triple A Program on quality of life (QoL) in a randomized controlled trial.

Methods: Students were from six high schools in rural Australia. The schools were randomly allocated to either the intervention or control group. 1379 students from Years 7 (12.5yrs) and 10 (15.5yrs) completed the ISAAC video questionnaire to measure asthma prevalence. Students who reported wheezing in the last year ( $n=272,86 \%$ R.R) completed the Paediatric Asthma Quality of Life Questionnaire and performed spirometry. Following the implementation of the program in the 3 intervention schools, these variables were re-assessed.

Results: Prevalence of recent wheeze was $25 \%$. At baseline, most students with recent wheeze reported mild to moderate impairment of QoL due to asthma. FEV 1 was $103 \%$ predicted and the FVCNC ratio was $89 \%$. Total QoL scores after adjusting for year and gender significantly improved in the intervention group when compared to the control group $(p=0.03)$ with $25 \%$ of students with asthma achieving a clinically relevant improvement, compared to $12 \%$ in the control group $(p=0.01)$. This effect was greatest in Year 10 students and in females. Significant improvements occurred in the activities domain ( $41 \%$ vs $28 \%, p=0.03)$ and some improvement in the symptoms domain $(26 \%$ vs $18 \%$ ) and the emotions domain (27\% vs $20 \%$ ), however these failed to reach statistical significance except for males in the emotions domain (39\% vs $19 \%, p=0.02$ ). Lung function improved significantly in both the control and intervențion groups. FEV $N$ NC ratio remained stable over time. Conclusion: The Triple A Program leads to a clinically and statistically important improvement in QoL, but not in lung function, in students with asthma. The challenge in the new millennium is dissemination and sustainability of the program.

ACTIVATION OF PROTEASE ACTIVATED RECEPTORS INDUCE CYTOKINE RELEASE FROM THE RESPIRATORY EPITHELIAL CELLS N Asokananthan', PT Graham1, DA Knight2, PJ Thompson² and GA Stewart' Department of Microbiology', Allergy and Asthma Research Unit, Dept of Medicine ${ }^{2}$ University of Western Australia, Nedlands, WA.

Asthma is a very common, chronic disease in both children and adults. The respiratory epithelium is important in asthma as it is the first tissue to contact inhaled allergens and contributes to the inflammatory response due to its ability to synthesise a range of pro-inflammatory products under the influence of a variety of stimuli. In this regard, we have shown that allergenic house dust mite proteases are potent inducers. What is not clear from our studies, however, is how the mite protease induced effects are mediated at the cellylar level. Recent identification of a novel family of G-protein coupled cell surface receptors which activate a variety of cellular functions, once cleaved by proteases, has been of particular significance in this study. Thus far, four PAR have been identified and differentiated on the basis of their activation by different proteases; PAR 1,3 and 4 are activated by thrombin whereas PAR-2 and 4 is activated by trypsin.

We have for the first time shown the presence of PAR in airway epithelial cell lines BEAS-2B, A549, $\mathrm{NCl}-\mathrm{H} 332$ and cultured human lung cells using specific antibodies. Further we have confirmed the presence of mRNA for these receptors. We used ELISA to determine the effect of the small peptides consisting of six amino acid residues, corresponding to the cleaved nascent $\mathrm{N}$ terminus portion of the receptor of PAR-1 to -4 in activating the receptors on the release of the pro-inflammatory cytokines IL-8, IL-6, and GMCSF from the lung epithelial cell lines. These results demonstrate that activation of PARS present on human airway epithelial cells will lead to the production of proinflammatory cytokines. Further, we have also shown that thrombin and trypsin are also capable of cleaving this protease-activated receptor. Thus, this study takes us a step closer to understanding the mechanism of action of the dust mite proteases and allergens on the lung epithelium.

Supported by the NHMRC \& Asthma foundation WA. Key words: Protease activated receptor, Cytokines, Allergy. 
NON-ALLERGIC PROTEINS FROM THE HOUSE DUST MITE - IMMUNE RESPONSES TO RECOMBINANT HOUSE DUST MITE FERRITIN IN ALLERGIC AND NON-ALLERGIC SUBJECTS

MJ Epton', L Hazell1, W Smith', PJ Thompson?', WR Thomas' (1) Department of Molecular Biology, TVW Telethon Institute for Child Health Research. P O Box 855 West Perth. WA 6872 and (2) Department of Medicine. University of Western Australia

The majority of proteins making up the house dust mite (HDM) are not allergenic. Immune responses to these proteins may alter responses to HDM allergens by changing the cytokine milieu around antigen presenting cells. Alms: To isolate and characterise HDM non-allergens, and to study the immune responses to recombinant non-allergens in allergic and non-allergic subjects. Methods: A Dermatophagoides pteronyssinus $\lambda$ gt11 library was screened with rabbit anti-HDM serum and antigenic clones screened for absence of IgE binding. A suitable clone was sequenced and expressed as a recombinant fusion protein. Immune responses to this protein were studied in allergic and non-allergic volunteers by immunoglobulin binding, peripheral blood mononuclear cell (PBMC) proliferative responses, and cytokine production. Results: A cDNA clone coding for ferritin, probably heavy chain, was isolated and a recombinant protein expressed using the pET vector. No IgE binding to the recombinant protein was seen from a battery of allergic sera. The protein induced significant PBMC proliferative responses (Mean proliferation (SE) $8811(1050) \mathrm{cpm}$ vs control $1265(149) \mathrm{cpm}(n=15$,

$p<0.0001)$. There was no difference between allergic and non-allergic groups in proliferative responses. Stimulated PBMC produced significant IL-5 (Mean IL-5 $106(20) \mathrm{pg} / \mathrm{ml}$ ) and interferon- $\gamma$ (Mean IFN- $\gamma 2077$ (751) pg/ml). There was no difference between allergic and non-allergic groups for either cytokine. Conclusions: House dust mite ferritin has been cloned, sequenced and expressed as a recombinant protein, which has the immunoglobulin binding characteristics of a non-allergen. Significant T-helper 2 type cytokine production is observed with PBMC stimulation. The classification of proteins as allergens or non-allergens may require more than just the ability to bind IgE.

Support: NH\&MRC

Key words: House dust mite, allergy, recombinant, ferritin, non-allergen Nominations for awards: Nil

INSECT VENOM IMMUNOTHERAPY - A 10-YEAR REVIEW OF THE USE OF A "RUSH PROTOCOL" IN AN AUSTRALIAN TEACHING HOSPITAL'S ALLERGY UNIT.

Westall GP, Czarny D, Thien FCK, O'Hehir RE, Douglass JA. Department of Allergy, Asthma \& Clinical Immunology, Monash University Medical School, The Alfred Hospital, Prahran VIC 3181.

Purpose of study: To report the 10-year cumulative experience at the Alfred Hospital with a rush venom immunotherapy protocol used on patients who have presented with anaphylaxis after insect venom stings. Methods: Patients were referred to the allergy unit after a history suggestive of anaphylaxis following insect venom sting. Skin tests and RAST levels confirmed causative insect. Patients were treated over a 5 -day period in a hospital providing full emergency resuscitation facilities. The schedule consisted of: Day 1 (0.1-0.4-12-4-8 $\mu \mathrm{g}$ of venom), Day $2(10-20-30-40 \mu \mathrm{g})$, Day $3(50-50 \mu \mathrm{g})$, Day 4 $(100 \mu \mathrm{g})$, Day $5(100 \mu \mathrm{g})$. Results: 68 venom-allergic patients received a total of 73 courses of rush immunotherapy. 65 were treated with honey bee venom, 7 with yellow jacket venom (vespula sp.) and 1 with paper wasp venom (polistes so.) The mean age was 38.02 years with a range 13-71 years. The severity of the initial sting was graded according to Müeller's scale: grade I $(2.94 \%)$, grade II $(16.18 \%)$, grade III $(27.94 \%)$, and grade IV $(52.94 \%)$. Of the 68 patients 24 were atopic and 10 were asthmatic. Of the 73 courses of VIT, complications occurred in 27 courses, of which 25 were hypersensitivity reactions. One patient was initially inadvertently given the incorrect venom (polistes instead of vespula wasp venom), and 2 patients developed gramnegative sepsis presumably, from infected intravenous sites. During induction 13 patients $(17.8 \%)$ developed severe generalised reactions. In all 949 injections were administered during the induction phase, and adrenaline was administered on 14 occasions (1.48\%). During the maintenance phase severe hypersensitivity reactions necessitating the use of adrenaline occurred in 4 patients. As a consequence of repeated severe generalised reactions during maintenance, One patient was advised to discontinue immunotherapy. Conclusions: The experience gained over 10 years of using a rush protocol during the induction phase of VIT suggests that this should continue to be performed in an in-patient setting with appropriate resuscitation measures. Of those patients receiving VIT, $89 \%$ were bee-allergic. This figure is different to that seen in Europe and the United States, where most patients are waspallergic.

\section{CHILDHOOD ASTHMA AND EXPOSURE TO FORMALDEHYDE AND} VOLATILE ORGANIC COMPOUNDS

Krassi Rumchev1, Steve Stick2, Jeff Spickett1, Mike Phillips'

(1) School of Public Health, Curtin University Perth 6845 and (2) Respiratory Medicine Department, Princess Margaret Hospital for Children

Young children spend most of their time indoors (80\%-90\%) and exposure to indoor air pollutants has been associated with increased acute respiratory disease morbidity, increased prevalence and incidence of respiratory symptoms and aggravation of asthma.

In a case-control study carried out in Perth, indoor environmental factors were studied in relation to respiratory illness in 192 children, aged between 6 months and 3 years old. Cases $(n=88)$ were children who attended the Accident and Emergency Department at Princess Margaret Hospital and were diagnosed with acute asthma. Controls $(n=104)$ were healthy children and identified from birth records through the Health Department. Information about respiratory conditions experienced by the child and characteristics of the home was collected in a self-administered questionnaire, completed by the child's parents/guardians. Formaldehyde, nitrogen dioxide, VOCs, house dust mites, temperature and humidity were monitored in each household. Skin prick tests were performed on all children. The unconditional logistic regression model showed that VOCs exposure was a statistically significant independent risk factor for asthma with OR=1.06 [95\% Cl: 1.01-1.10]. Furthermore, formaldehyde appeared to be a risk factor for asthma only among atopic children with $\mathrm{OR}=1.03$ [95\% $\mathrm{Cl}: 1.01-1.05]$. House dust mite levels were also a significant risk factor with $\mathrm{OR}=1.63,[95 \% \mathrm{Cl}$ : 1.08-2.48]. No association between asthma and nitrogen dioxide levels was seen. The study results showed that indoor environmental factors contribute as risk factors for childhood asthma.

Supported by the APAWS

Key Words: asthma, children, indoor environmental factors

Nominations for Awards: Nil

OUTPATIENT CLINIC DESENSITISATION FOR ASPIRIN SENSITIVE ASTHMA-RHINOSINUSITIS

Westall G, Thien F, O'Hehir R. Department of Allergy, Asthma and Clinical Immunology, Alfred Hospital and Monash Medical School, Commercial Road, Prahran 3181.

BACKGROUND: Aspirin sensitive asthma is a distinct syndrome affecting about $10 \%$ of asthma patients. Rhinosinusitis is a significant co-morbidity in these patients with minorrhoea, anosmia and recurrent nasal polyposis. Aspirin desensitisation has been found to improve nasal symptoms as well as reduce steroid requirements, reduce need for repeated nasal polypectomies, and fewer emergency presentations for asthma. However, currently published desensitisation protocols are time and labour intensive. AlM: To develop a protocol of outpatient clinic desensitisation for aspirin sensitive asthmarhinosinusitis suitable for a hospital or specialist ambulatory clinic. METHODS: Patients with asthma-rhinosinusitis with a history or suspicion of aspirin sensitivity were recruited from the Allergy Clinic of the Affred Hospital. Baseline lung function was obtained and patients were included if FEV1 was greater than $70 \%$ predicted. Patients were challenged in the clinic commencing with oral aspirin $50 \mathrm{mg}$ (half a Cardiprin ${ }^{\mathrm{TM}}$ tablet) and observed for 2 hours with half hourly peak flow and symptom records, and FEV1 at the end of challenge. If the challenge was negative, the patient returned at weekly intervals, and challenged with 100,200 and $300 \mathrm{mg}$ until a positive challenge. If the challenge was positive, then the patient was maintained at that threshold dose of aspirin daily until the next visit when they would be challenged at the next higher dose. A validated minoconjunctivitis quality of life (RQOL) questionnaire was obtained before and after desensitisation RESULTS: 9 patients $(5 \mathrm{M}, 4 \mathrm{~F})$ with mean age of $43 \pm 12$ years and mean baseline $\mathrm{FEV} 1$ $92 \pm 16$ (\% predicted) of were challenged. The threshold dose of a positive challenge was $50 \mathrm{mg}$ for 3 patients, $100 \mathrm{mg}$ for 4 patients and $200 \mathrm{mg}$ for 1 patient, and $300 \mathrm{mg}$ for 1 patient. The mean maximal fall in FEV1 was $12 \pm 9 \%$ with the lowest FEV1 reached being $66 \%$ predicted. One patient developed persistent urticaria after reaching $200 \mathrm{mg}$ and was withdrawn. The RQOL score fell from a median of 74 (range 26-131) predesensitisation to 30 (range 9-136) post-desensitisation. Patients were maintained on aspirin ranging from $100 \mathrm{mg}$ daily to $600 \mathrm{mg}$ bd. CONCLUSION: Gradual aspirin desensitisation is safe and feasible within an ambulatory hospital or specialist clinic setting, with the use of daily maintenance aspirin between visits to maintain the threshold. 
HOUSING FACTORS DETERMINING THE DISTRIBUTION OF ENDOTOXINS IN NEW ZEALAND HOMES

K. Wickens, J. Douwes, R. Siebers, G. Doekes", K. Mason, P. Fitzharris, J. Crane

Wellington Asthma Research Group, Wellington School of Medicine. Wellington, New Zealand; and Environmental and Occupational Health Group-, Wageningen, Netherlands

Recent reports suggest that bacterial endotoxin may be associated with asthma severity. Little is known about housing characteristics that influence the distribution of endotoxins in domestic dust. Endotoxin levels in dust were measured from a selection of houses with carpets in Wellington, New Zealand ( $n=74$ ). Using standard methods, we sampled dust from the whole living-room floor. Endotoxin levels were measured using a LAL assay. Questionnaires were used to collect information on home characteristics. The geometric mean level of endotoxin on the whole floor was 28,352 endotoxin units per gram of dust (EU/g), (Geometric $s d=3.4$ ). After adjusting for confounders, houses without insulation had significantly ( $p=0.008$ ) higher endotoxin leveis $(39,064 \mathrm{EU} / \mathrm{g}, \mathrm{sd}=4.0)$ than houses with insulation or a room or garage below the living room $(18,672 \mathrm{EU} / \mathrm{g}, \mathrm{sd}=2.3)$. Endotoxin levels were also independently higher $(p=0.04)$ in houses situated on sloping ground $(35,603 \mathrm{EU} / \mathrm{g}, \mathrm{sd}=3.7)$, compared with houses situated on flat ground $(20,252 \mathrm{EU} / \mathrm{g}, \mathbf{s d}=2.7)$, and in carpets which had been steamcleaned or shampooed in the last year $(52,351 \mathrm{EU} / \mathrm{g}, \mathrm{sd}=3.4)$, compared to carpets not steam-cleaned or shampooed $(25,348 \mathrm{EU} / \mathrm{g}, \mathbf{s d}=3.3)$, $(p=0.05)$. Houses without insulation or situated on sloping ground may have higher levels of endotoxins because they are likely to be damp or have poor underfloor ventilation. These are characteristics of many Wellington homes and it is in these homes that endotoxin levels may affect respiratory health. The higher levels of endotoxins in carpets which have been steam-cleaned or shampooed may also be due to an increase in moisture within these carpets atter cleaning.

\section{ASTHMA MORBIDITY AND MANAGEMENT AMONG ADULTS IN} AUSTRALIA, 1998

Gibson PG, Mitchell C, Bauman A, Henry R, Comino E, Robertson C, Laudau L, Ruttin R. National Asthma Campaign (NAC), South Melbourne, Australia.

The NAC conducted a third epidemiological study of primary school aged children and their parents in late 1998 to assess changes in asthma management. Methods: Three surveys were conducted in six major centres, with samples of 13086 parents in 1990, 13991 in 1993 and 16844 in 1998 . Response rates were $84 \%, 84 \%$ and $64 \%$. Parents reported their asthma symptoms, diagnosis, medication and management. Results: Parents were aged $37-38$ years (mean) across surveys. Rates of 12 month wheeze were similar across surveys, at $19.1 \%$ in $1990,18.1 \%$ in 1993 and $20.2 \%$ in 1998 , whilst diagnosed asthma rates were $7.1 \%, 7.2 \%$ and $8.8 \%$. In those with diagnosed asthma, use of any reliever medication declined over time $(89.7 \%, 89.5 \%, 83.1 \%)$ as did theophylline use $(20.5 \%, 3.9 \%$, $0.9 \%)$, preventer use declined in $1998(36.8 \%, 44.5 \%, 40.6 \%)$. There was a decline in the proportion with asthma who reported having any lung function test in the previous year $(40.2 \%, 44.4 \%, 32.5 \%)$, and having a peak flow meter $(18.7 \%, 29.9 \%, 16.8 \%)$, but the proportion with a written action plan increased $(14.0 \%, 19.9 \%, 25.6 \%)$. Smoking rates of those with asthma (22.2\%) were similar to those without asthma (23.9\%). However, of those with asthma and frequent symptoms $30 \%$ currently smoked, compared to $18.3 \%$ of those with less frequent symptoms. There were significant trends in asthma medication use and management across levels of educational attainment. Concluslons: Use of written action plans has increased but use of PFM and having lung function assessed has decreased from 1993. Regular bronchodilator use declined, with higher levels of preventer use maintained. These results were remarkably similar to trends in asthma management among primary school children.

Acknowledgments: National Asthma Campaign and participating centre research assistants.
ASTHMA PREVALENCE IN THE INTERNATIONAL STUDY OF ASTHMA COMMUNITY RESPIRATORY HEALTH SURVEY (ECRHS)

Soo Cheng, Neil Pearce, on behalf of the ISAAC Steering Committee and the European Community Respiratory Health Survey

Wellington Asthma Research Group.Wellington School of Medicine P.O. Box 7343. Wellington, New Zealand

International and regional prevalence comparisons are required to test and generate hypotheses about the causes of increasing asthma prevalence worldwide. The International Study of Asthma and Allergies in Childhood (ISAAC) is the first such study in children and the European Community Respiratory Health Survey (ECRHS) is the first such study in adults. We have therefore conducted a comparison of the findings from these two surveys, for the 17 countries in which both surveys were undertaken. There was a strong correlation between the ISAAC and ECRHS prevalence data, with $64 \%$ of the variation at the country level, and $74 \%$ of the variation at the centre level, in the prevalence of "wheeze in the last 12 months" in the ECRHS Phase I data being explained by the variation in the ISAAC Phase I data. There was also generally good agreement in the international patterns observed in the two surveys for self-reported asthma (74\% of country level and $36 \%$ of centre level variation explained), self-reported asthma before age 14 years $(64 \%$ and $26 \%)$, hay fever $(61 \%$ and $73 \%)$, and eczema $(41 \%$ and $50 \%$ ). These findings therefore add support to the validity of the two studies which provide a new picture of global patterns of asthma prevalence, and identify some of the key phenomena which future research must address.

Supported by the Health Research Council of New Zealand Key words: asthma prevalence epidemiology

MELBOURNE STUDY OF CHILDHOOD ASTHMA, OUTCOME AT AGE 42

Elisabeth Horak, Colin F. Robertson, Anna Lanigan, Liam Welsh, Mary Roberts, John Wilson, and Anthony Olinsky.

\section{Dept. of Respiratory Medicine, Royal Children's Hospital, Melbourne 3052}

In 1964, 295 wheezy children and 106 controls were recruited at age 7 years, to determine the outcome of childhood asthma. A further 83 children with severe asthma were included in 1967 at age 10 . The subjects have been reevaluated each 7 years with clinical history, lung function measurements, BHR and atopy status. In this report, we present the results at age 42. To date, clinical history is available for 328 subjects and lung function for 238. At recruitment, children were classified as: $C_{\text {, controls; }}$ MWB, mild wheezy bronchitis ( $<5$ episodes of wheezing with RTI); WB, wheezy bronchitis ( $>5$ episodes of wheezing with $R T$ ); $A_{1}$ asthma (wheezing unassociated with RTI); SA, severe asthma (persistent symptoms) At 42, the subjects were reclassified as: $W$-no wheeze in the last 3 years; $X-n o$ wheeze in the last 3 months; $Y$-wheezing in past 3 months, < once per week; $Z$-wheezing in past 3 months, > once per week. Data in the table represent the outcome at age 42 for each of the groups at age $7 / 10$.

\begin{tabular}{|c|c|c|c|c|c|c|c|}
\hline \multicolumn{2}{|c|}{ Classification } & W & $x$ & $\bar{Y}$ & $\mathrm{z}$ & FEV1(\%pred.) & FEF75-25(\%pred.) \\
\hline \multicolumn{2}{|c|}{ C $\quad(n=61)$} & $85 \%$ & $8 \%$ & $5 \%$ & $2 \%$ & $104 \%$ & $86 \%$ \\
\hline MWB & $(n=57)$ & $67 \%$ & $19 \%$ & $14 \%$ & $0 \%$ & $108 \%$ & $84 \%$ \\
\hline WB & $(n=73)$ & $59 \%$ & $12 \%$ & $18 \%$ & $11 \%$ & $102 \%$ & $83 \%$ \\
\hline A & $(n=88)$ & $33 \%$ & $18 \%$ & $26 \%$ & $23 \%$ & $96 \%$ & $70 \%$ \\
\hline SA & $(n=49)$ & $12 \%$ & $14 \%$ & $29 \%$ & $45 \%$ & $86 \%$ & $55 \%$ \\
\hline
\end{tabular}

These results show a good clinical outcome for childhood asthma into adult years with relative preservation of lung function. Only those with severe asthma in childhood have a modest reduction in lung function in adulthood. Overall, there has been little change in clinical status in the last 7 years and no further deterioration in lung function.

Supported by NATIONALBANK AUSTRIA and NHMRC

Key words: childhood asthma, outcome, lung function 


\section{PREVALENCE OF ASTHMA IN RECREATIONAL SCUBA DIVERS}

Paul Langton1, Carolyn Grove1,2, Rebecca Cresp², Emma Lalor² \& Liora Valinsky².

Departments of Medicine ${ }^{1}$ \& Public Health2, University of Western Australia, Nedlands, 6009, WA.

Asthma is considered a contributory factor in SCUBA-diving related mortallty and is present in up to $9 \%$ of deaths. Current asthma is regarded as a medical contra-indication to diving. Although routine screening aims to exclude asthmatics from dive training, some asthmatics are known to dive. The prevalence of asthma amongst divers, and in relation to diving mortality, is unknown. We aimed to determine the prevalence of asthma in a cohort of Western Australian recreational SCUBA divers. We sought to characterise their general health, to identify any diving related problems and to determine the beliefs of divers regarding the implication of asthma on dive safety. Methods: Questionnaires were mailed to 982 divers who had either recently completed a PADI diving course, or who were members of the University of WA dive club.

Results: In the $540(55 \%)$ respondents, the prevalence of current asthma was $9.7 \%(95 \%$ CI $7.1-12.3 \%)$. This not significantly different from the prevalence of $11.6 \%$ in Australian adults. Past asthma was present in $5.6 \%$ and a further $15.3 \%$ had symptoms suggestive of asthma. Most asthmatic divers reported only symptoms of mild airways disease. There were no significant differences in the general health of asthmatic and non-asthmatic divers. Asthmatics had not experienced any increase in diving related tochnical or health problems. Although most asthmatics identified asthma related diving risks, many continued to dive despite active disease. Conclusions: The prevalence of current asthma in divers does not support the contention that asthmatics are over represented in diving deaths. Current screening is ineffective at identifying asthma and precludes education of asthmatics in the potential risks of diving

Supported by the Asthma Foundation of WA.

Keywords: Asthma, SCUBA, diving

\section{IS ALLERGEN EXPOSURE THE MAJOR PRIMARY CAUSE OF} ASTHMA?

Neil Pearce, Jeroen Douwes, Richard Beasley

Wellington Asthma Research Group, Wellington School of Medicine, P.O. Box 7343. Wellington. New Zealand

In recent decades, a number of authors have argued that allergen exposure is the major primary cause of asthma, and that the global increases in asthma prevalence are due to increases in exposure to aeroallergens. We have assessed the epidemiological evidence in support of this hypothesis No longitudinal studies were identified in which allergen exposure during infancy in a random population sample has been related to asthma risk after age six years. Two studies have been conducted in selected populations chosen on the basis of a family history of asthma or allergy; one study found a non-statistically significant association whereas the other study found no association. Many of the identified prevalence studies in children showed negative associations between allergen exposure and current asthma, and the weighted averages of the population attributable risks in children were $4 \%$ for Der $p 1,11 \%$ for Fel d $1,-4 \%$ for Bla g II, and $6 \%$ for Can $f 1$. There was little change in these estimates in studies in which children whose parents had adopted allergen avoidance measures were excluded. Furthermore, evidence from population studies is equivocal and provides little consistent evidence that allergen exposure is associated with the prevalence of asthma at the population level. Population-based cohort studies are clearly required, but currently available evidence suggests that allergen exposure is at most a minor risk factor for the primary causation of asthma in children.

Supported by the Health Research Council of New Zealand

Key words: asthma, allergy, atopy, epidemiology

\section{PLACENTAL WEIGHT AND ITS RELATION TO INFANT SIZE AT BIRTH} AND ASTHMA AND ATOPY IN CHILDHOOD

Philip Leadbitter', Neil Pearce1, Soo Cheng', Malcolm R. Sears'2, David Holdaway ${ }^{3}$, Erin Flannery ${ }^{3}$, G. Peter Herbison ${ }^{3}$, Richard Beasley ${ }^{1}$

[1] Wellington Asthma Research Group. Department of Medicine. Wellingten School of Medicine, P.Q. Box 7343. Wellington. N.Z: [2] McMaster University, Hamilton, Ontario. Canada: [3] Dunedin School of Medicine. P.Q.Box 913. Dunedin, N.Z.

In a previous analysis we found an association between infant size at birth and the development of asthma and atopy in childhood. We have further analyzed the data to investigate factors influencing infant size at birth, and how they relate to asthma and atopy in later childhood. Methods: A longitudinal birth cohort of 1037 children born in Dunedin, New Zealand in 1972-1973 who were enrolled at age 3 years and were subsequently assessed every 2-3 years. Results: At age 13 years, data were available on 735 subjects ( $71 \%$ of the original cohort). Those infants with a placental weight of less than $460 \mathrm{gm}$ ( $10^{\text {th }}$ centile) had an adjusted odds ratio for reported asthma of $0.2(95 \% \mathrm{Cl} 0.1-0.9)$ at age 13 years. Placental weight was not associated with bronchial hyperresponsiveness or skin prick test positivity at 13 years, or serum total IgE at 11 years. The placental weight to birth weight ratio was not associated with reported asthma or any of the markers for atopy. Conclusions: Reduced placental growth is independently associated with a reduced prevalence of asthma at age 13 years. The placenta plays a crucial role in fetal nutrition and development and the factors influencing placental growth may also be "programming" the fetal immune or respiratory systems.

Supported by the Health Research Council of New Zealand Keys words: asthma, epidemiology, childhood

\section{INCREASED WEIGHT IN CHILDREN IS A RISK FOR SYMPTOMS AND MEDICATION USAGE FOR ASTHMA, BUT NOT AIRWAY HYPERRESPONSIVENESS.}

Linda M Schachter. Cheryl Salome, Jennifer Peat, Ann Woolcock, Institute of Respiratory Medicine, Sydney University, NSW, Australia

Obesity may be a risk factor for asthma in children. If so, the effect could be due to an effect of obesity on lung volume and thus airway hyperresponsiveness (AHR). Methods: Data from 5982 Caucasian children (8-11 years) from 7 large epidemiological studies performed in NSW were analysed. Subjects were included if data were available for height, weight, age and a measure of airway responsiveness (AR). Doctor diagnosis of asthma, history of wheeze, cough and medication usage in the last 12 months were obtained by questionnaire. Body mass index (BMI) $\mathrm{kg} / \mathrm{m}^{2}$, divided into quintiles per year age, was used as a measure of obesity. The highest quintile was defined as ovenweight. Dose response ratio (DRR) was used as a measure of $\mathrm{AR}$. DRR $=(\%$ fall in $\mathrm{FEV} / /$ ol histamine $)+3$. Alway hyperresponsiveness was defined as a DRR $\geq 8.1$. Adjusted odds ratios (OR) were obtained by logistic regression. Results: After adjusting for atopy, age, sex, family smoking history and family history, being overweight was a significant risk factor for cough in last 12 months (OR 1.35, 1.17-1.56, $p<0.001$ ) and medication usage for asthma in the last 12 months (OR 1.25, 1.08-1.44, $p=0.002$ ). Increased weight was not a risk for AHR (OR 0.89 , $0.75-1.05, p=0.14$ ), diagnosed asthma (OR 1.06, 0.92-1.21, $p=0.44$ ) or wheeze in the last 12 months (OR 1.11, 0.96-1.29, $p=0.16$ ). Conclusions: Increased weight in children is a risk factor for cough and medication use for asthma but not airway hyperresponsiveness.

This study was funded by The Asthma Foundation of NSW, Australia 


\section{MICRONUTRIENT INTAKE AND CURRENT ASTHMA IN YOUNG}

ADULTS.

Rosalie Woods', Rachel Stoney', Michael Bailey', Joan Raven², Paul Ireland4, Frank Thien ${ }^{3}$, Michael Abramson'1, Haydn Walters ${ }^{2}$ (1) Epidemiology \& Preventive Medicine (2) Respiratory Medicine (3) Allergy \& Clinical Immunology, Monash Medical School and Alfred Hospital, VIO 3181 (4) National Cancer Control Initiative. VIC 3053

Whilst the true role of diet in asthma remains unknown, it has been hypothesised that diet may be a risk factor for asthma. Current interest is focused on the protective effect of fresh oily fish (specifically, long chain omega 3 fatty acids) and Vitamin $E$ intake and the possible deleterious effect of dietary sodium. Methods: 638 young adults participated in a communitybased cohort study of dietary risk factors for asthma. Participants completed a detailed respiratory questionnaire and a subset $(n=419)$ also completed a semi-quantitative food frequency questionnaire and underwent methacholine challenge to measure bronchial hyper-reactivity (BHR). Predictive models were developed with multivariate logistic and linear regression. Results: 81 participants (38.9 (SD 6.3) years, $49 \%$ male) were defined as having current asthma on the basis of self-reported wheeze in the past 12 months and BHR. They had a BMI of $26.6(6.0) \mathrm{kg} / \mathrm{m}^{2}$ and $19 \%$ were current smokers. These characteristics did not differ significantly from those participants without current asthma ( $n=338$ ). Dietary sodium intake was a significant negative predictor $(\mathrm{OR}=0.03,95 \% \mathrm{Cl}=0.003-0.24)$, whilst tinned fish intake $(1.5,1.03-2.19)$, average portion size $(4.6,1.61-13.0)$ and total energy intake $(10.8,0.84-138)$ were independent predictors of current asthma. In a separate multiple regression model, dietary sodium was found to be a significant negative predictor of $\mathrm{PD}_{20}$, following adjustment for confounding variables (dietary cholesterol, atopy, and gender). Conclusions: Those with current asthma appear to consume more tinned fish and less dietary sodium, which may be consistent with dietary change consequent to asthma awareness and/or diagnosis. However, sodium intake was associated with BHR, suggesting that sodium may play a role in the dietary aetiology of asthma.

Supported by the NHMRC

Key words: asthma, bronchial hyper-reactivity, diet, epidemiology Nominations for awards: Nil

RECOMMENDED LENGTHO NOT REDUCE THE SUBSEQUENT RISK OF CHILDHOOD ASTHMA

A. Zacharasiewiczi, 2, T. Zidek ${ }^{1}$, G. Haidinger1, C. Vutuc ${ }^{1}$

1 Department of Epidemiology, Institute of Cancer Research. University of Vienna, Borschkegasse 8a, Austria.

2Wellington Asthma Research Group. Wellington School of Medicine RO. Box 7343 Wellington, New Zealand

Background/Methods: As part of the population - based ISAAC (International Study of Asthma and Allergy in Childhood) study conducted in Upper Austria we analysed breastfeeding habits and the influence on the development of asthma, allergic rhinitis and eczema in children up to the age of $6-9$ years. Results: $74.6 \%$ of the parents reported breastfeeding their infants: $25.9 \%$ were exclusively breastfed for less than 2 months, $25.9 \%$ for 2 to 4 months, $13.2 \%$ for $5-6$ months (the recommended duration of exclusive breastfeeding) and $10.4 \%$ of the children were exclusively breastfed for more than 6 months. Smoking during pregnancy and low educational level were inversely related to breastfeeding and duration of exclusive breastfeeding. in comparison to children without family history of atopic diseases, risk and high risk- children were exclusively breastfed more often and for a longer period of time. No significant reduction in the prevalence of lifetime diagnosis of asthma was found either for breastfed infants in comparison to those not breastfed (OR: 0.92; Cl: 0.82-1.04), nor did the length of exclusive breastfeeding have any significant influence on asthma prevalence; breastfeeding reduced the risk of allergic rhinitis (OR: $0.83 ; \mathrm{Cl}: 0.74-0.94$ ). For atopic eczema, breastfeeding and length of exclusive breastfeeding was associated with an increase in risk (OR: $1.23 ; \mathrm{Cl} 1.13-1.34$ ). Conclusion: A protective effect of breastfeeding was found only for allergic rhinitis. We found no significant effect of breastfeeding on asthma prevalence and even a negative influence on atopic eczema. Despite the otherwise undoubted advantages of breastfeeding for child nutrition, the importance of the recommended length of exclusive breastfeeding for allergy prevention should not be overemphasised.

Supported by Land Oberoesterreich and Department of Epidemiology, University of Vienna

Key words: breastfeeding, ISAAC, asthma, prevention, epidemiology

* Refer to page A18 for P39A

\section{ASTHMA IS NEGATIVELY ASSOCIATED WITH GROWTH IN HEIGHT} DURING ADOLESCENCE

Wei Xuan, Guy Marks, Brett Toelle, Elena Belousova, Jennifer Peat, Ann Woolcock

Institute of Respiratory Medicine, The University of Sydney, NSW 2006 Australia

Introduction and aim There has been concern about the effects of inhaled corticosteroids on the growth of children with asthma. Previous work has suggested that children with asthma who are not taking inhaled steroids grow normally (McCowan et al., 1998 BMJ 668-672). We have used data from the Belmont cohort to evaluate whether asthma is independently negatively associated with height and growth in height during adolescence and young adulthood.

Methods A cohort of 8-10 year old children ( $n=718$ ), living in Belmont, NSW, were studied at two yearly intervals from 1982-1992, and studied again in the period 1997-1999. At each survey, standing height was measured and information on wheeze and inhaled steroid use in the preceding 12 months was collected by questionnaire. The presence of airway hyperresponsiveness (AHR), defined as $\mathrm{PD}_{20} \mathrm{FEV}_{1}<3.9$ umol, was determined by histamine challenge test.

Results A history of wheeze in the last 12 months was associated with, on average, a $1.6 \mathrm{~cm}(95 \% \mathrm{Cl} 0.6$ to 2.6$)$ lower height but not with a lower growth in height $(P>0.45)$. The presence of AHR was associated, on average, with a $2.9 \mathrm{~cm}(95 \% \mathrm{Cl} 1.6$ to 4.1$)$ lower height and also with a lower growth in height $(P<0.001)$. The number of subjects using inhaled or oral steroid in the last 12 months at each survey ranged from 2 to 29.

Adjustment for steroid use did not affect the associations described above.

Conclusions People with asthma are slightly shorter in stature than others during adolescence and young adulthood and this is independent of inhaled or oral steroid use.

Supported by: NHMRC, Allen+Hanburys

Key words: Asthma, Epidemiology, adolescence

ASSOCIATION BETWEEN GENETIC VARIANTS OF THE MAST CELL CHYMASE GENE AND ATOPY IN PAEDIATRIC POPULATIONS.

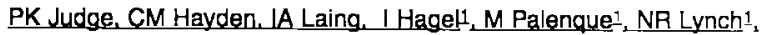
$\checkmark$ Goldblatt2, PN LeSouef.

Department of Paediatrics, University of Western Australia. 1'nstitute of Biomedicine, Central University of Venezuela, Experimental Asthma Clinic, Coche Island, Venezuela. ${ }^{2}$ Genetic Services of WA, Princess Margaret Hospital, Perth, Australia.

Atopic disorders are characterized by elevated serum immunoglobulin $\mathrm{E}$ (lgE) levels and are dependent on the specific triggering of IgE-sensitized mast cells by allergen. Mast cell chymase (MCC) has an impqrtant role in allergic inflammation within the respiratory tract and dermis. A previous study identified a polymorphism in the MCC gene (14q11.2), which can be detected by BstX1 enzyme restriction, and reported an association between this polymorphism and eczema. The aim of this study was to identify whether there was an association between genetic variants in the MCC gene and atopic outcomes in two unselected paediatric populations. Population 1: 77 six year old subjects from a cohort of infants recruited from an Australian hospital antenatal clinic. Population 2: 135 children (mean age 9 years) from Venezuela. Phenotypic parameters included skin prick testing, serum total \& specific IgE titres, blood eosinophil measurements and a questionnaire was administered to ascertain family history and environmental exposures in both populations. In addition, spirometry and histarnine challenge were conducted on population 1. In the Australian population, an association was found between the $B s t X 1$ polymorphism and specific $\lg E$ to mixed grass $(p=0.039)$. However, there was no association between this polymorphism and asthma, eczema or other atopic outcomes. In the Venezuelan population, the BstXI polymorphism was associated with a physician diagnosis of asthma $(p=$ 0.005 ), but not associated with elevated serum IgE levels or skin prick reactivity. These findings suggest that variants in the mast cell chymase gene are important in the genetics of atopy.

Funded by NH\&MRC (Australia) Medical Scholarship Key words: Atopy, genetics, polymorphisms, paediatrics 


\section{POLYMORPHISM SCREEN OF THE CC16 GENE PROMOTER REGION.}

Ingrid Laing', Jack Goldblatt², Peter Le Souëf'.

(1) Department of Paediatrics, Univerșity of Western Australia (2) Genetic Services. Princess Margaret Hospital for Children, WA, 6008.

The A38G polymorphism in the $5^{\prime}$ non-coding region of exon 1 of the Clara cell secretory protein (CC16) gene has previously been associated with an increased risk of developing asthma and reduced levels of circulating $\mathrm{CC} 16$ protein. Aim: To screen the promoter region of the $\mathrm{CC} 16$ gene to identify other polymorphisms that may alter CC16 gene function. Methods: Sixty four children were selected for asthma from a tertiary hospital respiratory clinic and 44 controls were recruited from a suburban general hospital. DNA from each subject was used in PCR to amplify the 550bp $\mathrm{CC} 16$ promoter region. Two PCR products, 287 and 273bp in length, encompassing the CC16 promoter region were screened for mutations using single stranded conformational polymorphism (SSCP) analysis and heteroduplex analysis. DNA sequencing on a $10 \%$ random sample was used to confirm these results. Results: No polymorphisms were identified in the CC16 promoter region. Conclusions: The association between the A38G polymorphism, asthme and reduced plasma $\mathrm{CC} 16$ levels is most likely due to attered $\mathrm{CC} 16$ gene function caused by the A38G polymorphism and not another linked polymorphism in the CC16 promoter region.

Supported by the NH\&MRC

Key words: Polymorphism screen, CC16, promoter, asthma. NomInations for Awards: nil

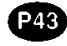

\section{$\beta_{2}$ ADRENOCEPTOR GENOTYPES AND LIFE-THREATENING ASTHMA}

Yang IA1, 3, Rabnott GC1, Tunnicliffe AM1, Douglas JA1, Shaw JG1, MCCarthy JB2, Zimmerman PV', Fong KM ${ }^{1}$

1Division of Thoracic Medicine and 2]ntensive Care Unit (ICU), The Prince Charies Hospital: 3Department of Medicine, University of Queensiand. Brisbane QLD 4032

The $\beta_{2}$ adrenoceptor ( $\beta_{2} \mathrm{AR}$ ) has key functions in asthmatic airways, and exhibits desensitisation and down-regulation with prolonged $\beta_{2}$-agonist exposure in asthmatics. Functionally relevant $\beta_{2} \mathrm{AR}$ polymorphisms have recently been characterised. lle-164 reduces $\beta_{2} A R$ affinity for $\beta_{2}$-agonists. Codon 16/27 polymorphisms enhance down-regulation, reduce bronchodilatation and increase noctumal asthma. 5 ' leader cistron polymorphism reduces receptor density and expression. We hypothesised that these $\beta_{2}$ AR polymorphisms modify asthma severity. Aim: To examine $\beta_{2} A R$ polymorphisms as disease-modifying genes in asthma. Methods: We recruited 53 adults with life-threatening asthma (admission to ICU due to asthma), 127 mild asthmatics (no ICU admission) and 177 healthy anonymous blood donors. $\beta_{2}$ AR genotypes were determined using PCR. Results: Patients with lifethreatening asthma had mean (SD) age 42 (18) years and $55 \%$ were female. The lle-164 variant was associated with an odds ratio of $6.9(95 \% \mathrm{Cl}$ 1.2-38.2) for life-threatening asthma (see Table). There was no association of codon $16 / 27$ or 5 'leader cistron polymorphisms with life-threatening asthma.

\begin{tabular}{|c|c|c|c|}
\hline Alleles & Life-threatening asthma & Mild asthma & Blood \\
\hline lle- 1 & $8 \%)$ & $(0.4 \%)$ & \\
\hline Thr- 16 & 102 (96.2\%) & $253(99.6 \%)$ & $352\left(99.4^{\circ}\right.$ \\
\hline
\end{tabular}

* $P=0.027$ (Fisher's exact test) compared with other groups

Conclusions: The lle-164 polymorphism in the coding region of the $\beta_{2} \mathrm{AR}$ gene was infrequent but increased the risk of life-threatening asthma. Correlation of $\beta_{2} \mathrm{AR}$ genotype to asthma phenotype has implications for understanding the pathogenesis of severe asthma, predicting prognosis and individualising asthma management.

Supported by: The Prince Charles Hospital Foundation, The Asthma Foundation of Queensland, NH\&MRC Medical Postgraduate Scholarship Key words: Asthma, $\beta_{2}$ adrenoceptor, polymorphism Nomination for Prlzes:
ASSOCIATION STUDY OF CC16 AND CD14 POLYMORPHISMS IN AN UNSELECTED POPULATION ASSESSED AT AGE 8 AND 25. AR O'Donnel|1. CM Hayden ${ }^{1}$ LA Laing ${ }^{1}$, B Toelle ${ }^{2}$. G Marks ${ }^{2}$, JK Peat?2. $\checkmark$ Goldblat ${ }^{3}$, PN Le Souef 1 .

1Department of Paediatrics, University of Western Australia, Perth, Australia 2Institute of Respiratory Medicine, New South Wales, Australia ${ }^{3}$ Genetic Service of WA, Princess Margaret Hospital, Perth, Australia.

The genes for Clara cell secretory protein (CC16) and CD14 both contain common polymorphisms (A38G and C-159T respectively) that have been associated with asthma-related phenotypes and altered serum levels of $\mathrm{CC}_{16}$ and $\mathrm{CD} 14$ respectively. Aim: To investigate the relationship between $\mathrm{CC} 16$ A38G and CD14 C-159T and the atopic phenotype in an unselected cohort. Method: 276 eight year olds were recruited from an unselected Australian population and followed-up at age 25. Assessment included questionnaire, spirometry, histamine challenge and skin prick test. Blood was taken at age 25 for DNA and total serum IgE. Genotypes were determined using restriction enzyme digestion. Independent association analyses were done relating genotype to phenotype at age 8 and 25. Results: CC16: 14\% were 38AA $50 \% 38$ AG and $36 \% 38$ GG. There was no association between CC16 genotype and asthma-related parameters (asthma diagnosis, symptoms, bronchial hyper-responsiveness (BHR), wheeze $+\mathrm{BHR}$ ) in subjects analyzed at age 8 and 25. CD14: $28 \%$ were $-159 \mathrm{CC}, 49 \%-159 \mathrm{CT}$ and $23 \%-159$ T. At age 8 there was a 2.5 fold lower risk of one $\geq 3 \mathrm{~mm}$ skin prick test in $-159 \mathrm{TT}$ subjects compared with $-159 \mathrm{CC}$ and $-159 \mathrm{C} T$ subjects $(95 \% \mathrm{C} \mid=1.04-5.88$, $p=0.039$ ). There were no associations between CD14 genotype and other atopic parameters. At age 25 there was no association between CD14 genotype and atopic parameters. Conclusion: A reduced risk of a positive skin prick test was found in 8 year old children with the CD14 -159TT genotype, but the association was of marginal statistical significance. No other associations were found between $\mathrm{CC} 16 \mathrm{A38G}$ or CD14 C-159T and asthma or atopic parameters in subjects age 8 or 25 years. These data suggest that these two polymorphisms do not have a significant role in inherited susceptibility to atopy or asthma in this population.

Funded by AstraZeneca

Key words: asthma, atopy, genetics, polymorphisms

FLUUTICASONE PROPIONATE INHIBITS EOSINOPHILIA, GOBLET CELL HYPERPLASIA AND AIRWAY HYPERRESPONSIVENESS IN A MURINE MODEL OF ALLERGIC BRONCHOCONSTRICTION

M Boujaoude, R Vlahos, DJ Fernandes, TL Bamford \& AG Stewart

Department of Pharmacology, University of Melbourne, Victoria 3010

Asthma is a disease characterised by the presence of chronic airways inflammation, airway hyperresponsiveness (AHR) and airway wall remodelling (AWR). AWR may explain a major part of AHR. We have previously reported that glucocorticoids inhibit the proliferation of human cultured airway smooth muscle (Br J Pharmacol 116, 3219-3226, 1995). Aim: To examine the effects of the glucocorticoid fluticasone propionate (FP) on the development of bronchoalveolar lavage (BALF) eosinophilia, AHR and AWR in a murine model of allergic bronchoconstriction. Methods: Male C57BL/6 mice were sensitised by intraperitoneal (ip) injection of ovalbumin (OVA) on days 0 and 12, followed by 8 aerosol challenges ( 30 min) with a combination of OVA ( $5 \% \mathrm{w} / \mathrm{v}$ ) and fetal calf serum (FCS, $5 \% \mathrm{v} / \mathrm{v}$ ) on days 20-27. FP (0.03-1 mg/kg) was administered daily by ip injection at least $1 \mathrm{~h}$ before OVAFCS challenge. Control mice were challenged with saline and received vehicle ( $3 \mathrm{ml} / \mathrm{kg}, 4 \%$ DMSO + $96 \%$ peanut oil) by ip injection. On days 19 and 27 , airway responsiveness to aerosolised methacholine ( $M C h, 3-12 \mathrm{mg} / \mathrm{ml}$ ) was measured using non-invasive, whole body plethysmography (Penh). On day 28, the mice underwent BAL with sterile saline and cytospot preparations were stained with DiffQuick for eosinophils. Results: FP, at all doses tested $(0.03-1 \mathrm{mg} / \mathrm{kg})$, reduced OVAFCS-induced increases in BALF eosinophils. The threshold for inhibition of goblet cell hyperplasia by FP was $0.1 \mathrm{mg} / \mathrm{kg}$ whereas only 1 $\mathrm{mg} / \mathrm{kg}$ FP significantly reduced OVAFCS-induced AHR. Conclusion: These results indicate that FP inhibits AHR, BALF eosinophilia and goblet cell hyperplasia induced by repeated allergen inhalation with different potencies.

Supported by Glaxo Wellcome (UK)

Key words: Airways hyperresponsiveness, fluticasone propionate, eosinophilia, goblet cell hyperplasia, airway wall remodelling Nominations for Awards: Nil 


\section{A COMPARISON OF THE IN VITRO PERFORMANCE BETWEEN THE FORADILE ${ }^{\circledR}$ AEROLIZER ${ }^{\circledR}$ AND OXIS ${ }^{\circledR}$ TURBUHALER $^{\circledR}$}

Hak-Kim Chan, Nora Y.K. Chew

Department of Pharmacy, University of Sydney, NSW 2006. Australia.

Eformoterol fumarate dihydrate is a long acting beta-2 agonist for bronchodilation in asthmatic treatment. To date, Foradile ${ }^{\circledR}$ Aerolize ${ }^{\circledR}$ and $\mathrm{Oxis}^{\circledR}$ Turbuhaler are the only dry powder inhalers available for the delivery of eformoterol. The two products differ in the formulation, the aerosol production mechanism and the device resistance to air flow. Our aim was to compare the in vitro performance of these two inhalers in producing the eformoterol aerosols. The particle size distributions of the two inhalers was assessed by a four-stage liquid impinger (plus filter) with a glass throat, at flows of $30-120 \mathrm{~L} / \mathrm{min}$. Eformaterol collected from the impinger was assayed by high performance liquid chromatography using UV detection at $240 \mathrm{~nm}$. Fine particles are those $\leq 4.4-6.8 \mu \mathrm{m}$ in the aerosols, depending on the air flow. At high air flows, 90 and $120 \mathrm{~L} / \mathrm{min}$, both inhalers produced similar amounts $(4 \mu \mathrm{g})$ of fine particles in the aerosol per dose discharged. This fine particle mass is about $30 \%$ of the label claim. As the flow was decreased to 30 and $60 \mathrm{~L} / \mathrm{min}$, both inhalers produced significantly less fine particles $(p<0.05)$, with the Oxis ${ }^{\circledast}$ Turbuhaler ${ }^{\circledast}$ producing lesser amounts than the Foradile ${ }^{\circledR}$ Aerolize ${ }^{\circledR}$. At a 'comfortable' inspiratory effort of 40 $\mathrm{CmH}_{2} \mathrm{O}$, the Foradile ${ }^{\circledR}$ Aerolize ${ }^{\circledR}$ would produce a significantly higher fine particle mass in the aerosols. We conclude that the in vitro performance of the two inhalers for eformoterol was equivalent at high but not at low air flows.

Supported by Novartis Australia

Key words: eformoterol, dry powder inhaler, Foradile ${ }^{\circledR}$ Aerolizer ${ }^{\circledR}$, Oxis $^{\circledR}$ Turbuhalerition

Nominations for Awards: Nil
A CROSS SECTIONAL STUDY OF EOTAXIN AND IL-5 IN THE BRONCHOALVEOLAR LAVAGE (BAL) OF ASTHMATICS ON DIFFERENT LEVELS OF INHALED CORTICOSTEROIDS.

B N Feltis, CWard, DW Reid, E M Glare, R Bish, R L Boyd, E H Walters Department of Respiratory Medicine and Monash Medical School, Affred Hospital INTRODUCTION: Eosinophilia is a key feature of clinical asthma. Two important cytokines that play a role in eosinophil biology are eotaxin and IL-5, but human data are limited and especially their responses to inhaled corticosteroid (ICS) regimes. HYPOTHESIS: That there is an increase in IL-5 and eotaxin in asthma and that ICS decreases the levels of these cytokines. AIMS: To measure eotaxin and IL- 5 in the BAL of asthmatic patients on varied steroid doses and contrast those levels with non-asthmatic controls. METHODS: We have developed sensitive $(>3 \mathrm{pg} / \mathrm{m} /)$ chemiluminescent ELISA techniques that can measure both eotaxin and IL-5 protein levels in BAL. These assays have been performed in a cross sectional study of 42 steroid naive asthmatics, 53 asthmatics on a low steroid dose $(<1000 \mu \mathrm{g} /$ day BDP), 25 asthmatics on a high steroid dose ( $>1000 \mu \mathrm{g} /$ day BDP) and 25 non-asthmatic controls, in whom a standardised $3 \times 60 \mathrm{ml}$ BAL had been performed. RESULTS: Our findings are summarised in the figures below:
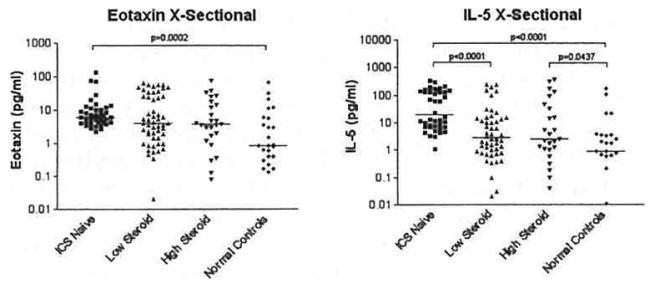

CONCLUSIONS: The above figures support our hypothesis that eotaxin and IL-5 are raised above normal levels in asthma. ICS appears to lower IL-5 expression to an intermediate level. In contrast raised eotaxin exhibits some resistance to steroid treatment across a range of doses. Future longitudinal studies will be required to investigate this further.

SUPPORTED BY: Glaxo - Wellcome and NHMRC

KEYWORDS: BAL Asthma IL-5 Eotaxin Treatment

EARLY INTERVENTION IN ASTHMA: EFFECTS OF FORMOTEROLBUDESONIDE "EARLY INTERVENTION" TH2 IMMUNITY AND INFLAMMATION IN A MURINE MODEL MURINE ASTHMA.

Alusha A. Mamchak, Jessica Jones, Sarah-Jane Beavitt, Alastair G. Stewart, Gary P. Anderson. Department of Pharmacology, University of Melbourne, Australia

Treatment with a combination of long acting beta-agonists and

glucocorticosteroids can control asthma symptoms/exacerbations (1), and early stercid intervention may produce better long term lung function (2). However, in vitro both steroids and beta2-agonists suppress IL-12 synthesis and may therefore reinforce deviation to a TH2 immune resonse (3). A TH2 immune response is associated with most childhood. As both steroids and beta2-agonists are presently used to treat asthma we examined their in vivo effects on the intensity of TH2 recall responses and eosinophilic inflammation elicted by ovalbumin (OA). Groups of 7-10 male $20 \mathrm{~g}$ Balb/c mice received nothing (C), PBS vehicle (PBS), high dose (based on species equivalence estimátes) daily exposure of formoterol $(F, 50 \mu g / k g$, sC), budesonide $(B, 30 \mu g / k g, s c)$ or both $(F / B)$

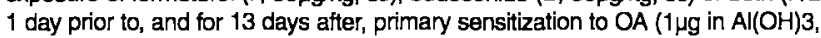
i.p.). The $O A$ response was boosted $14 \mathrm{~d}$ after primary sensitization to expand TH2 effectors, and the mice subsequently challenged with three exposures to OA $(1 \mu \mathrm{g} t \mathrm{n})$ on days 21,22 , and 23 to elicit recall, analysed on d24. Eosinophilic inflammation measured in BAL was not exacerbated compared to $C$ (4.0/0.9 E5 cells/ml - mean/SEM) and PBS (7.1/1.3 cells/ml) by F (6.6/4.6 E5 cells/ml) and was suppressed by $B$ or $F / B(0.7 / 0.2 \mathrm{E} 5,2.7 / 1.1 \mathrm{E} 5 \mathrm{celll} / \mathrm{m} / \mathrm{m}$, respectively, p<0.05). Compared to $C$ and PBS, $F, B$, and F/B suppressed IL-5 and IFNy levels in FACS sorted CD4+/CD3+ T cells obtained from draining lymph nodes and restimulated with anti-CD3 in vitro (IL-5: 450;540; 190; $<100$ and $<100 \mathrm{pg} / \mathrm{ml}$, respectively. IFNY: $650 ; 1100 ;<100 ;<100 ;<100 \mathrm{pg} / \mathrm{ml}$, respectively). In addition, B but not $F$ or $F / 8$ suppressed IL-5 production from anti-CD3 stimulated T cells from mixed BAL cultures. The data suggest that treatment with high dose parenteral $F, B$, or $F / B$ during emergence of $T H / 2$ immune response does not enhance the intensity of lung mucosal recall responses.

(1) Pauwels RA, et al. N Engl J Med 1997 337:1405-11; (2) Haahtela T, et al., N Engl J Med 1991 325:388-92; (3) Panina-Bordignon P, et al. J Clin Invest 1997 100:1513-9

Funded by an unrestricted grant from Astra-Zeneca, Sweden Key words: TH2, immune deviation, eosinophil, inflammation, formoterol, budesonide 
EFFECT OF BUDESONIDE ON PERCEPTION OF BREATHLESSNESS IN ASTHMATIC SUBJECTS

CM Salome, AM Roberts, SI Ware, HK Reddel, CA Jenkins, GB Marks and AJ Woolcock. Institute of Respiratory Medicine. University of Sydney, NSW Australia

The perception of breathlessness is an indirect determinant of asthma treatment, particularly with symptom based self-management plans. The effect of treatment on perception has received limited study. Aim: To determine the effect of inhaled budesonide on the perception of breathlessness induced by histamine challenge. Methods: 61 subjects with poorly-controlled asthma were randomised to receive budesonide 1600 or $3200 \mu \mathrm{g} /$ day for 8 weeks, followed by 8 weeks on $1600 \mu \mathrm{g} /$ day and subsequent down titration according to a clinical algorithm. Histamine challenges were performed at baseline and $8,16,24,48$ and 72 weeks. In 35 subjects the severity of breathlessness was recorded by Borg scores during challenge and perception was estimated as the slope of $\mathrm{Borg} / \mathrm{FEV}$ \% \%all. Blood eosinophils and serum ECP were measured. Results: Slope Borg/ FEV increased significantly after 8 weeks budesonide, $(0.11 \pm 0.02$ to $0.175 \pm 0.03, p<0.0001)$. Perception remained significantly increased at all subsequent visits, but did not increase any further. Slope Borg/FEV ${ }_{1}$ at baseline did not differ significantly between subjects on inhaled corticosteroids (ICS) at study entry and those not on ICS ( $0.11 \pm 0.03$ vs $0.11 \pm 0.02$ ). The magnitude of change in perception in the first 8 weeks of treatment did not differ significantly between the two treatment groups, and was not related to changes in baseline FEV , PD20FEV1, blood eosinophils or serum ECP. Conclusions: Perception of breathlessness is increased within 8 weeks by treatment with inhaled corticosteroids in asthmatic subjects. However, there is no further change during a year of good asthma control. The increase is not related to improvements in clinical or inflammatory markers. Altered perception during treatment with inhaled steroids may lead to underestimation of symptomatic improvement, with consequences for self-management.

Study support: NHMRC Australia, AstraZeneca Sweden and AstraZeneca Australia

Key words: asthma, perception, inhaled corticosteroids

\section{IDENTIFICATION OF LYN KINASE AS A CENTRAL REGULATOR OF SEVERE, PERSISTENT ASTHMA IN MICE.}

S.J. E. Beavitt (1) P.D. Sly (3), K. Harder (2), S. Brennan (2), D. Sly (3), J. Jones (1), A. Dunn (2), M. Hibbs (2), M. Smyth (4), D. Tarlington (5), G.P. Anderson (1).

(1) Dept. Pharmacology University of Melbourne. Parkville VIC 3052: (2) Ludwig Institute for Cancer Research, Parkville VIC 3052: (3) TWV Institute of Children's Health Research. Perth. WA 600Q: (4) Austin Research Institute, Heidelberg VIC 3084: (5) The Walter \& Eliza Hall Institute of Medical Research, Parkville VIC 3052.

The Src family tyrosine kinase, lyn, is currently thought to be a key therapeutic target in allergic asthma because it is essential for in vitro signalling by high affinity IgE receptors, the common $\beta$ chain of IL-3-, IL-5- and GM-CSF-receptors and the $B$ cell receptor complex. We used lyn knock-out mice (lyn-KO) (Hibbs et al., 1995) to test this hypothesis. Paradoxically, sensitized lyn-KO mice (C57BI6xC129; |Ab) mounted profound, persistent eosinophilic inflammation to ovalbumin aerosols (wildtype control $1.04 \pm 0.39 \times 10^{5}$ vs lyn KO $9.73 \pm 1.88 \times 10^{5}$ bronchoalveolar lavage eosinophils/ml, $n=11-12, p<0.001$ ), and had serum hyper-IgE, mast cell hyper-degranulation and bronchial hyperresponsiveness to inhaled antigen. The intense eosinophilia was not due to enhanced eosinophil progenitors (CFU-eos) in bone marrow or spleen. Similarly, elevated IgE was not responsible because double knock-out in B cell deficient (lyn $+\mu \mathrm{MT}$ KO) mice also showed increased eosinophilia. Although purified thoracic $\mathrm{CD} 4+$ lymphocytes secreted $>10$-fold higher levels of IL-4 and IL-5 (>100pg/m/ $/ 2 \times 10^{5}$ cells, $p<0.01$ ), but not IFN-y, ultrapurified CD4+ T cells did not express lyn. However, lyn was highly expressed in the rare NK1.1+ natural killer (NK) cell population. Depletion of NK cells with monoclonal antibodies, but not inactivation by gene targetting of their principle effector molecule, perforin, prevented development of the multi-trait asthma phenotype. These data represent the first example of a single molecular defect causing an asthma-like syndrome involving multiple, diverse and seemingly unrelated disease loci simultaneously. We propose that lyn links these diverse loci by a fundamentally important, undiscovered, biochemical mechanism governing asthma severity and progression, acting principally on the NK1.1+ lymphocyte subset. Hibbs et al., (1995) Cell: 83:301-311

Funded by NHMRC

Key words: lyn, TH2, IgE, NK cell, eosinophil, inflammation,

\section{CELLULAR LOCALISATION OF INTERLEUKIN-13 RECEPTOR IN NORMALS AND ASTHMATICS.}

T.L.Bamtord1 D.M.Smallwood ${ }^{1.2}$,T.Harris ${ }^{1}$,M.C.F.Pain ${ }^{2}$ J.W.W. Wilson ${ }^{3}$, C.Robertson4, A.G.Stewart 1 . Departments of 1 Pharmacology, The University of Melbourne, ${ }^{2}$ Thoracic Medicine, Royal Melbourne Hospital, ${ }^{3}$ Respiratory Medicine, Alfred Hospital, ${ }^{4}$ Respiratory Medicine, Royal Children's Hospital, Melbourne Australia.

Interleukin (IL)-13 appears to be capable of inducing the entire allergic asthma phenotype. Goblet cell hyperplasia, mucus hyperproduction and subepithelial fibrosis have been demonstrated in response to IL-13 in murine models of allergic bronchoconstriction. However, there are no studies of the distribution of IL-13 receptor in human ainways. We studied the localisation of the IL-13 receptor- $\alpha 1$ (IL-13R $\alpha 1)$ in the airways of a cohort of normals and asthmatics that have been followed prospectively with reassessment every 7 years since the age of 7 (H Oswald et al, $B M J$ 1994; 309: 95-6) and have recently returned for extensive review (age 42 years) including basic lung function tests and bronchoscopy. Endobronchial biopsies were analysed immunohistochemically for IL-13R $\alpha 1$ from 10 asthmatics of varying severity and 6 nonasthmatic controls. Fibroblasts were isolated from separate biopsies taken at the same time and cultured in DMEM $20 \%$ FCS and bFGF 3nM) for up to 5 weeks to produce an explant culture. Second passage cells were immuno-stained for IL-13R $\alpha 1$. IL-13R $\alpha 1$ was detected in basal epithelial cells, eosinophils, mononuclear cells and fibroblasts within the biopsies. IL-13R $\alpha 1$ was also identified in fibroblasts cultured from biopsy specimens. We conclude that the expression of the IL-13R $\alpha 1$ on cells associated with key asthma pathology is consistent with emerging evidence of IL-13 in a role for perpetuating the inflammatory response in asthma.

This work was funded by NH\&MRC Australia, Glaxo-Welcome UK.

THE RELATIONSHIP BETWEEN SUB-BASEMENT MEMBRANE THICKNESS AND AIRWAY WALL DIMENSIONS IN ASTHMA.

\author{
AL James, G Pearce-Pinto, J Elliot, N Carroll.
}

Department of Pulmonary Physiology, Sir Charles Gairdner Hospital. Nedlands 6009. Western Australia.

Airway remodelling in asthma includes increased wall thickness, areas of smooth muscle and mucous glands and deposition of extracellular matrix proteins and results in altered airway function. It is thought to be due to airway intlammation and responds to therapy with inhaled corticosteroids. Measuring airway inflammation and remodelling may be necessary to optimise therapy. Airway dimensions can only be measured on whole transverse sections. Aim: To determine if the sub-basement membrane thickness (BMt), which can be measured on a bronchial biopsy, reflects changes in dimensions across the entire airway wall. Methods: Transverse sections of large airways ( $>10 \mathrm{~mm}$ internal perimeter) from cases of fatal asthma ( $F A$, clinically severe), nonfatal asthma (NF, mild) and control (C) cases ( $n=5$ each) were examined. BMt was measured at $X 400$ and related to the area of the inner airway wall (WAi), outer wall (WAo), total wall (WAt), smooth muscle (ASM), mucous glands (Agl), cartilage (Acart) and numbers of eosinophils (Eos). Results: The mean value for BMt was stable after 15 measurements, taken randornly round the airway perimeter at intervals of $0.1 \mathrm{~mm}$. Mean BMt was $5 \mu \mathrm{m}$ in $\mathrm{C}$ and increased in NF $(7 \mu \mathrm{m})$ and FA (9 $\mu \mathrm{m}$ ) cases. BMt correlated positively with WAi (as \% of total cross-sectional area, $p<0.05)$, ASM $(p<0.001), \mathrm{Agl}(\mathrm{p}<0.001)$ and Eos in the inner $(p<0.05)$ and outer (0.001) airway wall for all cases grouped and, for the FA group alone, with ASM $(p<0.05)$ and Agl $(p<0.05)$. BMt was not related to BM length, muscle shortening. Acart, WAo or WAt. These findings suggest that $\mathrm{BMt}$ is a marker of airway remodelling in asthma, particularly in severe cases and that changes in BMt may be useful to monitor asthma treatment. Support: NHMRC Key words: Sub-basement membrane, airway remodelling, asthma. Awards: nil 
INHIBITION OF DEEP INSPIRATION (DI) DURING METHACHOLINE INHALATION INCREASES AIRWAY NARROWING IN ASTHMATICS.

Greg King, Barbara Moore and Peter Paré. Institute of Respiratory Medicine, Sydney, Australia and UBC Pulmonany Research Laboratory, Vancouver. Canada.

Introduction: Inhibition of DI during repeated methacholine inhalation increases airway narrowing in normals reaching a plateau at 10 minutes ( $G$. King et al, Am J Resp Crit Care Med 1999). Since the bronchodilator response to Dl is impaired in asthmatics, we hypothesised that inhibition of DI would have less of an effect on aiway narrowing during methacholine inhalation in asthmatics compared with normals.

Methods: We studied 4 male and 3 female asthmatics (age range 27-53 years) who received $5 \times \mathrm{PC} 15$ doses of methacholine given 5 minutes apart and measured FEV1 after each dose. On 4 separate days, they were given 2, 3, 4 or 5 doses in random order, but FEV1 was measured only at baseline and after the last dose. Dls were inhibited between times.

Results: Geometric mean PC15 was $2 \mu$ mol. When Dls were allowed, the maximum decrease in FEV1 occurred after 3 doses and was $22 \pm 5.7 \%$ of baseline. When DIs were inhibited, \% decrease in FEV1 was enhanced which became greater as the duration of inhibition of DI increased. The \% decrease after the last dose was $39 \pm 6.8 \%$ when Dls were inhibited compared with $19 \pm 2.5 \%$ when Dls were allowed

Conclusion: Inhibition of DI during methacholine inhalation in asthmatics increases airway narrowing by a similar degree in asthmatics and normals.

Support: Astra/MRC/PMAC Canada Fellowship.

Key words: Asthma, Airway smooth muscle, Deep inspiration
EXPRESSION OF MATRIX METALLOPROTEINASES-2 AND - 9 IN SERUM AND BRONCHOALVEOLAR LAVAGE FLUID OF ASTHMATIC PATIENTS.

G. King, M. Tamm, A. Woolcock, J. Black, M. Roth. Institute of Respiratory Medicine, University of Sydney, Australia

INTRODUCTION: Asthma is characterised by thickening of the basement membrane and altered mechanical properties of the airway. This may be caused by enhanced proliferation of bronchial smooth muscle cells and an accumulation of extracellular matrix (ECM). Matrix metalloproteinases (MMP) with gelatinolytic activity are key regulators of local remodelling of ECM and have been suggested to be involved in the pathogenesis of asthma. We investigated the expression pattern of MMP-2 and MMP-9 in the serum of healthy controls and in serum and bronchoalveolar lavage fluid obtained from asthmatic patients.

RESULTS: Based on enzymatic activity by gelatine zymography, the inactive pre-cursor of MMP-2 was highly expressed in asthmatic serum samples, while it could not be detected in controls. Similar, high amounts of active MMP-2 were present in asthmatic serum samples, while only a low expression of active MMP-2 was observed in controls. The MMP-9 pre-cursor was more often expressed in sera obtained from asthma patients, while only few controls expressed low amounts of the MMP-9 pre-cursor. Active MMP-9 was not detectable in any serum sample. Interestingly, the active forms of both MMP were highly expressed in bronchoalveolar fluid samples of asthmatics.

CONCLUSION: We observed a distinct expression of MMP-2 and MMP-9 in the serum and BAL of asthmatic patients. It is yet unclear whether the enhanced expression of these MMP contributes to disease progression or if it represents a remodelling process of the lung to reconstitute the structure of its ECM. Support: Fairfax Family Foundation Grant.

Key words: Asthma, Airway mechanics, Matrix metalloproteinases

\section{BRONCHODILATATION AFTER DI IS REDUCED IN ASTHMATICS COMPARED WITH NORMALS ONLY AFTER GREATER DEGREES OF AIRWAY NARROWING.}

Gregory G. King, Alyson M. Roberts, Wei Xuan and Cheryl M. Salome. Institute of Respiratory Medicine. University of Sydney, NSW 2006. Australia.

INTRODUCTION: It has been suggested that bronchodilatation after deep inspiration (DI) is reduced in asthmatics. The relationship between the magnitude of narrowing and amount of reversal has not been determined. We hypothesized that in normals, $\mathrm{DI}$ induced bronchodilatation is greater as narrowing increases whereas in asthmatics, the bronchodilatation fails to increase with greater narrowing.

METHODS: 16 normals and 18 asthmatics (airway hyperresponsiveness and wheeze) inhaled doubling doses of methacholine up to a maximum dose of $8 \mu \mathrm{mol}$. The difference between flow at $40 \% \mathrm{VC}$ on complete (V40c) and partial (V40p) expressed as the \% baseline V40p (V40DI) was measured, as was the decrease in V4Op as percent baseline ( $\triangle \mathrm{V} 40 \mathrm{p})$. The relationships between $V 40 D I$ and $\triangle V 40 p$ were examined using a mixed effects linear regression model.

RESULTS: The mean \pm SEM $\%$ decrease in $\triangle V 40 p$ was $49 \pm 4 \%$ in normals and $70 \pm 3 \%$ in asthmatics $(p<0.01)$ and for FEV1 was $8 \pm 1 \%$ and $21 \pm 2 \%$ respectively $(p<0.01)$. The linear regression slopes of $V 40 D I$ vs $\triangle V 40 p$ were $0.58(p<0.01)$ in normals and $0.06(p>0.05)$ in asthmatics.

CONCLUSIONS: The reversal of bronchodilatation due to $\mathrm{DI}$ increases to match increasing airway narrowing in normals but does not similarly increase in asthmatics. This difference could be due to mechanical differences of the airway walls or functional differences in airway smooth muscle.

Support: Institute of Respiratory Medicine and NH\&MRC Australia.

Key words: Asthma, Airway smooth muscle, Deep inspiration
DEEP INSPIRATION (DI) PRECEDING HISTAMINE INHALATION PROTECTS AGAINST AIRWAY NARROWING BY REDUCING BASELINE AIRWAY SMOOTH MUSCLE TONE.

G King, C Salome and P Paré. Institute of Respiratory Medicine, Sydney, Australia and UBC Pulmonary Research Laboratory Vancouver, Canada.

INTRODUCTION: DI that preceds methacholine inhalation by 6 mins or less reduces airway narrowing. We hypothesized that the Dl prior to histamine inhalation protects against airway narrowing by reducing baseline airway smooth muscle tone.

METHODS: Four normal subjects inhaled histamine in a døse estimated to decrease the FEV 1 by $15 \%$, under 4 different conditions; with and without DI for 10 mins preceding inhalation either with or without $80 \mathrm{mg}$ ipratropium administered 25 mins before inhalation. FEV1 was measured 5 times after histamine inhalation.

RESULTS: When DI's were inhibited before histamine inhalation, $\triangle F E V 1$ was greater compared to when Dl's were taken $(30 \pm 3$ cf $20 \pm 4, p=0.05$, ANOVA). Pre-treatment with ipratropium decreased $\triangle F E V 1$ when DIs were inhibited $(30 \pm 3$ of $12 \pm 1, p<0.05)$ but had no effect on $\triangle F E V 1$ when Dls were not inhibited.

CONCLUSIONS: Pre-treatment with ipratropium abolished the increase in $\triangle F E V 1$ caused by inhibition of DI before histamine inhalation. DI may protect against induced airway narrowing by reducing baseline airway smooth muscle tone.

Support: Institute of Respiratory Medicine and Astra/MRC/PMAC Canada Fellowship.

Key words: Asthma, Airway smooth muscle, Deep inspiration 
EFFECT OF A LONG-ACTING $\beta_{2}$ AGONIST OVER 3 MONTHS ON AIRWAY WALL VASCULAR REMODELLING IN ASTHMA. BE Orsida, C Ward, X Li, R Bish, JW Wilson, F Thien*, EH Walters Respiratory Medicine. The Alfred Hospital and Monash Uriversity Medical School, Melbourne. Victoria 3181 ("now at Department of Allergy and Clinical Immunology).

There are little data regarding the potential effects of anti asthma treatment on indices of airway remodelling such as airway vascularity in asthma patients. Methods: We studied 45 symptomatic asthmatic subjects who were receiving treatment with low dose inhaled corticosteroids and 28 non asthmatic normal subjects as a control population. Subjects underwent bronchoscopy with ainway biopsy and asthmatic subjects were then randomised to receive supplementary inhaled salmeterol $50 \mathrm{\mu g}$ bd, fluticasone propionate $100 \mathrm{\mu g}$ bd or placebo for 3 months. Biopsy of the airway was then repeated. The biopsies were analysed for vascular structures in the sub-epithelial lamina propria. Results: Sufficient biopsy material was available for analysis of vascularity in 34 of the asthmatic and 25 of the normal subjects. We confirmed that asthmatic airways had a significant increase in the number of vessels $/ \mathrm{mm}^{2}$ of lamina propria compared to normal airways $\left(524 \pm 137\right.$ vessels $/ \mathrm{mm}^{2}, n=34$ vs $425 \pm 130$ vessels $s / \mathrm{mm}^{2}, n=25 ; p=0.004$ ). As previously described decrease in vascular area with low dose ICS at baseline was evident (Orsida et al. Thorax 1999; 54: 289-295). There was a decrease in the density of vessels of lamina propria after supplementary treatment only in the salmeterol group compared to baseline (before, $535 \pm 153$ vessels $/ \mathrm{mm}^{2}$ vs after, $400 \pm 142$ vessels $/ \mathrm{mm}^{2} ; n=12 ; p=0.04$ ). There was no significant change in the fluticasone $(n=11)$ or placebo $(n=11)$ treatment groups.

Discussion: No treatments were associated with adverse affects on parameters of airway vascularity. The demonstrated fall in vessel number following salmeterol treatment may suggest an advantageous effect of long acting $\beta_{2}$ agonists on this manifestation of airway remodelling over the 3month time scale of this study. This effect seems to be complementary to the action of ICS on airway vascularity.

Supported by: Glaxo-Wellcome, Australia.

Key words: Asthma, airway remodelling, vascularity, treatment.

\section{PLASMA PHOSPHOLIPASE $A_{2}$ (PLA $)$ ACTIVITY IN STABLE ASTHMATICS AND CONTROL SUBJECTS}

\author{
Nenad Petrovic, Carolyn Grove and Philip Thompson
}

Department of Medicine University of Western Australia, and Asthma and Allergy Research Institute. Perth, Wesstern Australia

PLA $\mathrm{A}_{2}$ represents a large group of enzymes that share as a common characteristic the capacity to hydrolyse fatty acids from the sn-2 position of glycerophospholipids. Hydrolysis of phospholipids by $\mathrm{PLA}_{2}$ provides the precursors for eicosanoid synthesis that is important in lung inflammation and asthma. We have determined secreted PLA $A_{2}$ activity levels in plasma of a cohort of 64 stable asthmatics ( 43 mild and 21 severe) as well as in 23 contro subjects. Correlation between plasma $P\left\llcorner A_{2}\right.$ activity values and gender, age, atopy, \% of predicted PEF (\%PEF) and vitarnin C levels was analysed. Variables were compared by either unpaired, two-tailed t-test or by linear regression analysis. PLA $A_{2}$ levels were significantly higher in males compared to females $\left(p=0.0287\right.$ ). Levels of $P L A_{2}$ negatively correlated with plasma vitamin $C$ concentrations $(p=0.0080)$. Furthermore, negative correlation with vitamin $C$ levels and asthma status was also significant $(p=0.0066)$. Positive correlation between \%PEF and PLA $A_{2}$ was observed in control and mild asthmatics $(p=0.0420$ and 0.0389 respectively) but no such correlation was observed in severe asthmatics $(p=0.2378)$. In male subjects $P L A_{2}$ levels correlated significantly with age $(p=0.0253)$ and atopic status $(p=0.0481)$ while in females no significant correlation among these parameters was observed. Preliminary results of this study suggest that effects of dietary anti-oxidants and plasma $P L A_{2}$ on lung inflammatory processes might be gender-dependent. Further studies are needed to characterize the relationship between vitamin $C$ and $\mathrm{PLA}_{2}$ levels as well as the effect of gender on these measurements.

Supported by Asthma Foundation of Western Australia

Key words: PLA $A_{2}$ Asthma, vitamin $C_{1}$ gender.

NomInations for awards: $\mathrm{Nil}$
GLYCOLMETHACRYLATE EMBEDDING OF TISSUE CAN BE USED FOR MEASURING COLLAGEN III AND IV WITHOUT SIGNIFICANT LOSS OF ANTIGENICITY.

M.Pais, A.Sama, C.Ward, B.Feltis, E.Haydn Walters.

Respiratory Medicine. Monash University And Alfred Hospital. Melbourne VIC.

Ten Hacken et al. (Mod Pathol 1997;10:1043-1046) suggested that antigenicity levels for cell markers decreased alarmingly over time in bronchial biopsy specimens that were embedded in glycolmethacrylate (GMA), which has taken on 'gold standard' status in recent years. This raised the question of whether the same would occur when looking at indices of airway wall remodelling such as collagen III (scar tissue) and collagen IV (vessels) staining. This prospective study was undertaken to see what changes occurred to levels of these collagens over time, as a prelude to a long term study of airway remodelling in asthma. Nasal polyps were collected from 8 patients and embedded in GMA after appropriate processing. Sections were stained for collagen III and IV (after etching in acetone for 15 minutes and then digesting in $0.001 \%$ trypsin to improve staining) and then scored using computerised image analysis where collagen IV yielded a score of vessel number expressed per $\mathrm{mm}$ of basement membrane and collagen III was expressed as percentage positive area. Survey sections were measured to $100 \mu \mathrm{m}$ below the basement membrane. Sectioning, staining and scoring were carried out at baseline (base; 1 day post polypectomy) and subsequently at 1 month (TP1), 2 months (TP2) and 3 months (TP3) after embedding. Mean \pm SD are presented:

\begin{tabular}{|l|l|l|l|l|}
\hline & Base & TP1 & TP2 & TP3 \\
\hline Vessel no & $19.0 \pm 6.4$ & $19.1 \pm 6.3$ & $24.9 \pm 13.2$ & $19.4 \pm 7.1$ \\
\hline $\begin{array}{l}\text { Col IV } \\
\text { (No/mmBM) }\end{array}$ & $0.6 \pm 0.05$ & $0.5 \pm 0.3$ & $0.5 \pm 0.3$ & $0.7 \pm 0.2$ \\
\hline $\begin{array}{l}\text { Col III } \\
\text { (\%area) }\end{array}$ & $40 \pm 10$ & $40 \pm 6$ & $40 \pm 10$ & $40 \pm 10$ \\
\hline
\end{tabular}

CONCLUSION:Our results indicate that embedding in GMA does not appear to cause a systematic loss of antigenicity over time for collagen III and collagen IV.

Key words: Glycolmethacrylate, Nasal Polyp, Collagen III, Collagen IV Supported by:Glaxo Wellcome Australia.

PROLIFERATION CAPACITY OF BRONCHIAL SMOOTH MUSCLE CELLS IS ENHANCED IN ASTHMATICS

M. Roth, P Johnson, G King, M Tamm, J Black

Department of Pharmacology and Institute of Respiratory Medicine, University of Sydney, Australia

Histopathological features of airways in patients with asthma include airway inflammation and bronchial smooth muscle cell (BSMC) hyperplasia. It is not yet clear if this is due to increased cell proliferation or delayed cell apoptosis. Establishment of primary human cell lines from the lung provides a unique basis to study pathogenetic mechanisms of lung diseases on a cellular level. To our knowledge no data are published on primary bronchial smooth muscle cells cultured from asthmatics. To investigate cell proliferation, BSMC from three patients with asthma were cultured and compared with BSMCs from non-asthmatic non-sensitised controls. Cells were grown under sterile conditions in RPMl supplemented with $10 \%$ fetal calf serum (FCS), $8 \mathrm{mM} \mathrm{L}$ glutamine and antibiotics. Cells were seeded onto 24 well plates ( $1 \times 10^{4}$ cells $/ \mathrm{cm}^{2}$ ) and grown until reaching a logarithmic growth phase. Cell cultures were kept in FCS free medium for 48 hours. Following serum deprivation cells were restimulated with $10 \%$ human serum (obtained from atopic patients with asthma or non-sensitised healthy controls). Cell counts were performed daily for the following 4 days. In addition, proliferation was assessed by ${ }^{3} \mathrm{H}$ - thymidin incorporation. Asthmatic cells grew more rapidly compared with controls. Cell growth increased by a mean of $87.3 \%$ if sensitised serum was used compared with an increase of a mean of $48.6 \%$ under non-sensitised conditions. Asthmatic cell lines exhibited a greater increase in cell proliferation under sensitised conditions compared with control cells. Summary: In this study we found evidence that bronchial smooth muscle cells of patients with asthma grow faster compared with controls. This effect was more apparent if cells were grown under sensitised conditions. Increased cell proliferation might contribute to airway thickening and altered contractility, which is observed in asthma. 
EXACERBATIONS OF ALLERGIC BRONCHOPULMONARY ASPERG|LLOSIS (ABPA) A STUDY OF LABORATORY MARKERS Caldecott M. ${ }^{1}$ James $\mathrm{H}^{2}{ }^{2}$ Cook R. ${ }^{2}$ Gillis D. ${ }^{2}$, Rice A. ${ }^{1}$,Holmes M. ${ }^{1}$ Woodcock J.2. Scicchitano R. ${ }^{2}$.

(1)Department of Thoracic Medicine. Royal Adelaide Hospital. (2) Division of Human Immunology, Institute of Medical and Veterinary Science, Adelaide

Introduction: A reliable disease marker for acute exacerbations (AE) of $A B P A$ is not available. Currently the diagnosis is made clinically based on symptoms, radiology, total IgE and eosinophil counts. IL-5 plays an important role in eosinophil accumulation in the airways of patients with ABPA. The $\alpha$ chain of the IL-5 R exists as membrane bound and soluble isoforms. Hypothesis: Changes in the ratio of membrane to soluble $\alpha \mathrm{lL}-5 \mathrm{R}$ (M/S $\alpha$ IL-5R) correlate with disease activity. Aims: To compare the usefulness of M/S $\alpha \mid L-5 R$ with other markers to predict AE of ABPA. Methods: We developed an assay to measure the isoforms of $\alpha \mathrm{IL}-5$ R RNA in peripheral blood leukocytes. Patients were followed prospectively for 6 months. AE were diagnosed clinicaliy and were managed by the treating physician. We serially measured total eosinophil count, total IgE, Aspergillus specific $\lg E$ and eosinophil cationic protein (ECP). RNA for the isoforms of the $\alpha$ lL-5R was measured in peripheral blood using RT-PCR with real time PCR technology (Taqman). Results: 9 AE were observed in 10 patients. All $A E$ were treated with prednisolone. There was a significant correlation between eosinophil counts and disease activity $(P<0.01)$. An increase in ECP with exacerbations was also observed, but was not significant $(P=0.09)$. IgE was a poor marker of disease activity. While, as a group, there was no significant change in mean $M / S \alpha$ LL-5R ratios with $A E$, in one patient the ratio varied inversely with eosinophil count. Conclusion: Eosinophil counts, but not total IgE, were a reliable marker for $A E$ in ABPA. We intend to carry out further studies on M/S $\alpha$ LL-5R in a larger group, including the effects of corticosteroids on this ratio.

Key words: Allergic bronchopulmonary aspergillosis, Total $\lg E$, interleukin-5

\section{EFFECT OF ANAESTHESIA AND SURGERY UPON EXHALED NITRIC} OXIDE AND CARBON MONOXIDE.

R. Farhadieh, P.S. Thomas. Faculty of Medicine, UNSW, \& Department of Respiratory Medicine. Prince of Wales Hospital, Randwick 2031.

Increased exhaled nitric oxide is associated with inflammatory changes in the lung and airway, and likewise elevated exhaled carbon monoxide is also associated with asthmatic inflammation in non-smokers. Volatile anaesthetic agents and intubation are known irritants of the major ainways. These agents might therefore be expected to affect the regulation of nitric oxide synthases in the upper airway. We therefore studied subjects who were undergoing abdominal surgery, principally gynaecological, to assess the changes in exhaled nitric oxide before and after surgery and a general anaesthetic. 36 subjects agreed to participate, 2 had asthma, 11 were current smokers. All had exhaled nitric oxide measured using a gas impermeable bag and a flow restriction to increase oral pressure to approximately $5 \mathrm{~cm}$ of water, thus avoiding nasal contamination of the sample. Sampling was repeated 24-30 hours later as soon as the subject felt able to provide a specimen.

Results: Mean nitric oxide level before surgery: $15.1+/-9.5 \mathrm{ppb}$; post surgery: $15.2+/-8.0 p p b$. Mean exhaled carbon monoxide levels before surgery (excluding smokers) 4.2+/-3.7ppm; post surgery: $4.9+/-4.1 \mathrm{ppm}$. None of these changes was significant when log transformed as appropriate and subjected to parametric statistical tests. Conclusion: Modern anaesthetic agents and abdominal surgery do not appear to affect exhaled levels of nitric oxide or carbon monoxide.

Key words:exhaled nitric oxide, exhaled carton monoxide
THE EFFECTS OF SALIVARY CONTAMINATION ON SPUTUM CELL COUNTS: ESTABLISHING QUALITY CRITERIA

Naomi L Timmins, Jodie L Simpson, Kellie Fakes, Peter G Gibson

Airways Research Centre, John Hunter Hospital, Newcastle, NSW Australia

Salivary contamination confounds the accurate interpretation of induced sputum cell counts in asthma. The acceptable levels of contamination and its effect on measurements are not well established. The aim of this study was to examine the effects of salivary contamination on cell counts and to establish quality markers that could be used to determine the adequacy of sputum samples. Healthy adults $(n=6)$ provided samples of blood and saliva. Granulocytes were isolated using percoll gradient centrifugation and ammonium chloride lysis of red cells. Aliquots of saliva were added to the granulocyte suspension in the following concentrations ( $v \%): 0 \%, 10 \%$, $30 \%, 50 \%, 70 \%, 80 \%$ and $100 \%$. A total cell count and viability were periormed on the saliva-granulocyte suspension with cytospin slides prepared for differential cell counts and supernatant assayed for ECP. Saliva contained between 25 and $128 \times 104 / \mathrm{mL}$ cells per $\mathrm{mL}$, of which $25-44 \%$ were viable. The cellular differential of saliva contained 65 to $86 \%$ squamous cells, with the remainder being neutrophils. No eosinophils or lymphocytes were seen in saliva samples. The concentration of ECP in saliva ranged from 5 to $60 \mathrm{ng} / \mathrm{mL}$. The addition of increasing volumes of saliva to the granulocyte suspension had no significant effect on total and differential counts up to $50 \mathrm{vol} \%$. Salivary contamination at $70 \mathrm{vol} \%$ was associated with a squamous cell count of $>50 \%$. There was significantly reduced cell viability from $98.9 \%$ to $59 \%$ (p<0.01), eosinophil percentage from $15 \%$ to $7 \%$ $(p=0.01)$, lymphocyte percentage from $1 \%$ to $0 \%(p=0.01)$ and ECP from $241 \mathrm{ng} / \mathrm{ml}$ to $37 \mathrm{ng} / \mathrm{ml}(p<0.01)$. There was a non-significant trend for neutrophils to increase with the addition of saliva $(p=0.08)$. In conclusion, salivary contamination has little effect on the cellular differential until there are $>50 \%$ squamous cells present. There are significant effects on fluid phase markers at all levels of contamination. We propose quality criteria for sputum samples of $>90 \%$ viability and $<50 \%$ squamous cells in order to minimise the confounding effects of salivary contamination. More research is required to control for the effects of salivary contamination on fluid phase markers.

Supported by NHMRC

\section{AIRWAY EOSINOPHILIA IS ASSOCIATED WITH WHEEZE BUT IS UNCOMMON IN CHILDREN WITH PERSISTENT COUGH AND FREQUENT CHEST COLDS}

Ruth C Toneguzzi, Peter G Gibson. Jodie L Simpson, Anita C Chalmers. Peter AB Wark, Amanda J Wilson, Michael J Hensley

Airways Research Centre, John Hunter Hospital NSW and Newcastle Environmental Toxicology Research Unit, University of Newcastle Callaghan NSW

The role of eosinophilic airway inflammation in the variant asthma syndromes of cough and chest colds is not well defined. We tested the hypothesis that children with persistent cough and chest colds have increased sputum eosinophils, similar to those with wheeze, The parents of 390 primary school children completed a symptoms questionnaire. Children with wheeze $(n=25)$, cough $(n=12)$, recurrent chest colds $(n=17)$ and no symptoms (controls, $n=24$ ) consented to allergy skin prick tests, spirometry, combined hypertonic saline inhalation challenge with sputum induction, peak expiratory flow (PEF) and symptom diary over a 2 month period. Children with wheeze had significantly reduced PEF $(p=0.001)$ and higher sputum eosinophils when compared to the cough, chest cold and control groups (Medians: $3.1 \%$ vs $0.5 \%, 0 \%, 0 \%, \mathrm{p}=0.03$ ). The prevalence of eosinophilic bronchitis (sputum eosinophils $>2.5 \%$ ) was $45 \%$ in the wheeze group which was signiticantly higher than the control group $(9.35 \%, p=0.04)$. Eosinophilic bronchitis was present in two children with cough (17\%) and two with chest colds $(12 \%, p>0.05$ vs control). In these groups eosinophilic bronchitis was not associated with AHR ( $p>0.05)$ but tended to be associated with rhinitis. Children with cough and chest colds reported greater exposure to environmental tobacco smoke. In conclusion, this community-based survey of children with chronic respiratory symptoms has shown that wheeze is a good discriminator for the presence of eosinophilic bronchitis: persistent cough and recurrent chest coids without wheeze should not be considered variants of asthma.

Funding: Asthma NSW, Community Health \& Anti-Tuberculosis Assoc, NHMRC and NSW Health. 
INCREASED EOSINOPHILIC AIRWAY INFLAMMATION WITH CHLAMYDIA PNEUMONIAE INFECTION IN ASTHMA

J Simpson, PG Gibson, R Scicchitano, I Town. P Thomas, R Toneguzzi. I McNamara, P Mullins.

Airways Research Centre, John Hunter Hospital NSW. Royal Adelaide Hospital SA, University of NSW. Canterbury Respiratory Research Grous. Christchurch. NZ

Infection with Chlamydia pneumoniae (CP) is associated with increased asthma severity. The relationship between $\mathrm{CP}$ infection and airway inflammation in persistent asthma is not known. We sought to compare airway inflammation in asthmatics with and without serologic evidence of persistent or prior $\mathrm{CP}$ infection. Adults with symptomatic asthma were recruited and evaluated by clinical assessment, spirometry, and airway responsiveness to hypertonic saline and induced sputum analysis. Serum antibodies $(\lg G, \lg A)$ to $C P$ were measured by MIF and subjects classified as seronegative (CP-neg; $n=37$ ), seropositive to $\lg G$ alone (CP-G; $n=28$ ), and seropositive to both $\lg G$ and $\operatorname{IgA}(C P-G A ; n=53$ ). Subjects had moderate severity asthma with mean $\mathrm{FEV}_{1} 75 \%$ predicted and mean dose of beclomethasone $1300 \mu \mathrm{g}$ daily. ${ }^{*} p<0.05$

\begin{tabular}{|l|l|l|l|}
\hline & CP neg & CP-GA & CP-G \\
\hline Sputum eos $\%$ & 4.1 & 2.6 & $6.98^{*}$ \\
\hline ECP,ng/ml & 1802 & 1718 & $6458^{*}$ \\
\hline $\mathrm{KL}-5, \mathrm{pg} / \mathrm{ml}$ & 21 & 11 & $59^{*}$ \\
\hline $\mathrm{IL}-8, \mathrm{pg} / \mathrm{ml}$ & $6 \overline{0}$ & 94 & 223 \\
\hline
\end{tabular}

Sputum neutrophils and total cell counts were similar between the groups ( $p>0.05$ ).

In conclusion, asthma with Chlamydia pneumoniae infection and $\mathrm{CP}-\mathrm{G}$ seropositivity is characterised by an increase in the severity of IL-5 mediated eosinophil inflammation and eosinophil degranulation. An IgA response in association with $\operatorname{lgG}$ seropositivity to CP appears to protect against some of the inflammatory changes in asthma. Persistent $\mathrm{CP}$ infection may increase the severity of eosinophilic airway inflammation in asthma.

Supported by Hoechst Marion Roussel

\section{P67}

CHLAMYDIA PNEUMONIA AND ACUTE ASTHMA: PREVALENCE AND EFFECT ON AIRWAY INFLAMMATION

Peter AB Wark, Jodie L Simpson, Michael J Hensiey and Peter G Gibson Airways Research Centre, Department of Respiratory Medicine, John Hunter Hospital NSW Australia

Chiamydia Pneumoniae (CP) causes acute respiratory tract infections and persistence of the agent is seen in chronic severe asthma. Aims: To determine the prevalence of acute or reactivating infection with $\mathrm{CP}$ in acute asthma and it characterises the effect on airway inflammation. Methods: Adults ( $n=54$ ) presenting to the emergency room with acute exacerbations of asthma had a clinical assessment, spirometry and sputum induction, acutely and four weeks later. Paired serum samples were taken for chlamydia pneumoniae (CP) IgG and IgA and tested using ELISA. Results: Initial serum demonstrated a prevalence $\mathrm{CP} \operatorname{lgG}$ of $41 \%(n=22)$ and $C P \lg A$ of $31 \%(n=17)$. Ten (18.5\%) showed an increase in IgG and $21(38.5 \%)$ showed an increase in IgA consistent with either acute infection or reactivating infection. Those with rising $\mathrm{CP} \lg \mathrm{A}$ had more intense airway inflammation in sputum (median total cell count $\times 10^{6} ; 11.4$ ), compared to those with negative or stable CP IgA $(2.4, p<0.01)$. In addition there was an increase in levels of eosinophilic cationic protein (median 7071.1 vs 1016.7 $\mathrm{ng} / \mathrm{mL}, \mathrm{p}=0.02$ ) in sputum supernatant. An increase in serum igG did not significantly influence aiway inflammation (median total cell count 4.6 vs $2.8, p=0.3$ ) and eosinophilic cationic protein (median 2087.9 vs 1269.1 , $p=0.6$ ) though there was a trend similar to that seen in those with an increase in serum IgA. Conclusion: In acute severe asthma, serological evidence of acute or reactivating infection with $\mathrm{CP}$ was seen in $18 \%$ (rise in $\mathrm{IgG}$ ) and $39 \%$ (rise in IgA antibodies) of subjects. Acute asthma exacerbations accompanied by a rise in CP IgA had more intense airway inflammation with marked eosinophil degradation.

Supported by Asthma NSW

* Refer to page A18 for P67A
MODIFICATION OF ALLERGIC INFLAMMATION WITH BACTERIAL LIPOPOLYSACCHARIDE

MK Tulic \& PD Sly Clinical Sciences, TVW Telethon Institute for Child Health Research \& University of WA Department of Paediatrics. Perth WA 6008 Alms: To determine the influence of infective inflammation on response to allergen we have administered nebulised lipopolysaccharide (LPS) a) during the primary sensitisation phase, b) during allergen challenge or c) between the acute and late phase of the allergen response using an in vivo animal model. Methods and Results: During the primary sensitisation of PVG rats to ovalbumin (OA), a single aerosol challenge of LPS $(50 \mathrm{\mu g} / \mathrm{ml})$ given day -1 or up to day 4 after sensitisation, completely abolished allergic sensitisation, resulting in no increase in $\mathrm{lgE}$ or response to OA challenge. Presence of LPS $(0.5 .5$ or $50 \mu \mathrm{g} / \mathrm{ml})$ with $\mathrm{OA}$ in the same nebuliser during allergen challenge resulted in an immediate decrease in lung function (time-to-peak decreased from $10.0 \pm 0.9(\mathrm{SEM})$ to $2.5 \pm 0.2$ minutes $(n=6, P<0.001)$ but abolished latephase hyper-responsiveness, cellular influx and vascular leakage (assessed by Evans Blue dye) in a dose-dependent manner $(n=5, P<0.01)$. Exposure of sensitised animals to LPS, 18 hours post allergen challenge also inhibited the late-phase hyper-responsiveness when measured at 24 hours $(n=5, P<0.05)$ but further exacerbated the $O A$-induced neutrophil influx and Evans Blue leakage $(n=5, P<0.01)$. In the later group of animals, aminoguanidine (iNOSselective: $100 \mathrm{mg} / \mathrm{kg} \mathrm{sc}$ ) was effective in reducing the cellular influx and vascular leakage $(n=5, P<0.001)$ whilst L-NAME (cNOS-selective: $100 \mathrm{mg} / \mathrm{kg} \mathrm{sc}$ ) potentiated the allergen-induced hyper-responsiveness $(n=5, P<0.01)$. Conclusion: These results suggest that the modification of allergic response by LPS is dependent on the dose and timing of exposure. Whilst LPS exposure prior to sensitisation inhibits lgE production and hence the development of allergic inflammation, exposure post allergen challenge further exacerbates the OA-induced cellular inflammation in rats. In this model, nitric oxide production by iNOS plays a major role in the migration of inflammatory cells and vascular permeability following allergen challenge while that produced by cNOS limits bronchial hyper-responsiveness.

Supported by The Asthma Foundation of WA and NH\&MRC

Key words: allergic inflammation, bacterial infection, sensitisation, nitric oxide

Posters - Cell Biology/lmmunology

IL-13 ACTIVATES HUMAN CULTURED AIRWAY SMOOTH MUSCLE THROUGH THE IL-13 R $\alpha 1$ RECEPTOR

I. L. Bamford1, E. Guida1, T. Harris' J.W. Wilson² A. Nash ${ }^{3}$ and A.G. Stewart1. 'Department of Pharmacology, The University of Melbourne, 2Respiratory Medicine, Alfred Hospital, Prahran, and 3AMRAD Operations Pty Ltd, Victoria, Australia.

Animal models have shown that IL-13, produced by activated $\mathrm{CD}^{+} \mathrm{TH}_{2}$ cells, plays a key role in the pathophysiological features of allergic asthma. The function of IL-13 in human cultured airway smooth muscle cells (HASM) has been elucidated by investigating the presence of the receptor for IL-13 (IL-13R $\alpha 1$ ), its regulation at a transcriptional level and the effect of IL-13 on cell proliferation. HASM were grown to confluence in standard culture flasks and 8 chamber slides, and then serum-deprived for $24 \mathrm{hr}$ before a 30 minute pretreatment with either fluticasone propionate (FP $1 \mathrm{nM}$ ) or 2 -

methoxyestradiol (2-Meo 10 $\mu \mathrm{M}$ ) prior to incubation in cytokine mix (TNF $\alpha$ $0.03 \mathrm{nM}+\mid \mathrm{L}-1 \alpha 0.1 \mathrm{ng} / \mathrm{ml}$ ) or medium alone. Immunoperoxidase staining was used to determine the presence and location of the IL-13 R $\alpha 1(n=3)$. The expression levels of 2 mRNA transcripts for the IL-13 R $\alpha 1$ (4.2kb and $2 \mathrm{~kb}$ ) were assessed by northern Blotting ( $n=6)$. DNA synthesis was assessed by incorporation of $3 \mathrm{H}$-thymidine to determine growth potential of $\mathrm{IL}-13$ in the presence and absence of $5 \%$ FCS $(n=6)$. IL-13R $\alpha 1$ was detected in cultured HASM by immunohistochemistry. The increased staining intensity for IL$13 R \alpha 1$ following addition of the cytokine mix was accompanied by redistribution of immunoreactive $\mathrm{LL}-13 \mathrm{R} \alpha 1$ from cytoplasm to a distinctly perinuclear location. The intensity and redistribution of IL-13R $\alpha 1$ were reduced by pretreatment with either FP or 2-Meo. The level of the 2kb mRNA transcript was increased by the cytokine mix and decreased upon the addition of either FP or $2-M e O(69.8 \pm 5.0 \& 60.2 \pm 12 \%$ of the cytomix level, respectively). IL-13R $\alpha 1$ concentration-dependently stimulated DNA synthesis with additive effects with $5 \%$ FCS. These data are compatible with a functional influence of IL-13 on HASM.

This work was funded by NH\&MRC (Australia) 
CULTURE OF AIRWAY SMOOTH MUSCLE ON A COLLAGEN TYPE I MATRIX IMPAIRS THE ANTI-PROLIFERATIVE ACTION OF GLUCOCORTICOIDS

JV Bonacci, T Harris, TL Bamford, V Koutsoubos \& AG Stewart Department of Pharmacology, University of Melbourne, Victoria 3010 Changes in extracellular matrix (ECM) and airway wall rigidity are integral aspects of asthmatic airway wall pathology. The ECM in the subepithelial space of the asthmatic airway is characterised by excessive collagen deposition, which together with airway smooth muscle (ASM) stiffness, increase airway wall rigidity. Reduced distensibility of asthmatic airways limits stretch of ASM cells beyond resting state, reducing airway strain. Alm: We have investigated whether two important pathological features of asthma, increased amounts of ECM collagen type I, and reduced strain, affect ASM proliferation and its regulation by the glucocorticoid, dexamethasone. Methods: Bovine and human ASM were seeded in Flexce|l ${ }^{T M}$ plates containing a flexible silastic membrane coated with collagen I or laminin. A 72 h period of $4 \%$ stretch of the silastic membrane was applied at a frequency of 15 per minute, to mimic the strain expected in situ (A.JRCCM 158, S176, 1998). Cell numbers were determined by haemocytometry. Results: Neither matrix type nor level of strain significantly affected bovine ASM proliferation. However, following $72 \mathrm{~h}$ incubation with bFGF ( $300 \mathrm{pM}$ ), cells subjected to reduced strain and seeded on collagen were significantly more responsive to bFGF-induced mitogenesis (Laminin/strain 100; Laminin/no strain 129 \pm 9 ; Collagen/strain 142 \pm 20 ; Collagen/no strain 157 $\pm 20, P<0.05, n=6-7$ ). Similar effects were seen with human ASM cells. In cells seeded onto collagen and subjected to reduced strain, dexamethasone significantly inhibited bFGFinduced proliferation in bovine ASM cells (Control 100; bFGF 127 \pm 9 ; Dex/bFGF 82 $\pm 9, P<0.05, n=6$ ). However, human ASM cultured under the same conditions, were resistant to the anti-proifferative effects of dexamethasone. Conclusion: Proliferative responses of ASM appear to be biomechanically regulated.

Supported by Glaxo Wellcome (UK) \& NHMRC (Australia)

Key words: airway smooth muscle, strain, collagen, laminin Nominatlons for Awards: Nil

\section{EOSINOPHILIC INFLAMMATION AND SURFACTANT IN AIRWAYS OF CHILDREN WITH ASTHMA}

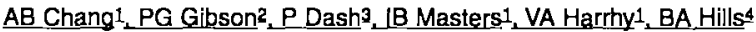

'Dept. of Respiratory Medicine, ${ }^{3}$ Dept. of Anatomical Pathology, ${ }^{4}$ Paediatric Respiratory Research Centre, Mater Misericordiae Children's Hosp, Brisbane; and 2Dept. of Respiratory Medicine, John Hunter Hospital, Newcastle, Australia.

Eosinophilic inflammation is a major component of asthma. Biologically, the physical barrier properties of surfactant (SAPL) that is predominantly dipalmitoyl phosphatidylcholine (DPPC) has been increasingly recognised. The relationship between SAPL and inflammation indices of asthma is unknown. We hypothesised that the degree of inflammation influences DPPC content of induced sputum. Sputum was induced from 21 children (16 asthmatics [A], 5 controls [C]) using the hypertonic saline and analysed for eosinophil cationic protein (ECP), myeloperoxidase (MPO) IL-8 and DPPC. The standard spectrophotometric method of quantifying DPPC content of SAPL was used. Blood was also analysed for eosinophil count and ECP. The median age of children ( 16 boys, 5 girls) was 10.7 years (range 6-16.9). Multiple linear regression on log data revealed a significant relationship between sputum ECP and DPPC level (ug per $\mathrm{ml}$ sputum) for group [A] $(r=0.766, p=0.0014)$, as well as for the total group $(r=0.638, p=0.0044)$. Log DPPC values per $\mathrm{ml}$ of sputum was significantly higher in group [A] when compared to group $[C]$, mean difference $0.48, p=0.033(95 \% \mathrm{Cl} 0.05,0.92)$. There was no relationship between sputum DPPC with sputum MPO sputum IL-8, serum ECP, serum eosinophils and sputum eosinophils. None of the clinical markers of asthma related to any inflammatory marker. We conclude that increased DPPC levels occur when eosinophilic inflammation is increased.

Study supported by The Sylvia \& Charles Viertel Charitable Foundation and HM Foreman Memorial Fund.

\section{LYMPHOID AGGREGATES AND HLA-DR EXPRESSION WITHIN THE AIRWAYS IN ASTHMA AND CHRONIC OBSTRUCTIVE PULMONARY DISEASE.}

N Carroll', S Mutavdzic', J Elliot'1, K Mackay², A James'.

(1) Dept Pulmonary Physiology, Sir Charles Gairdner Hospital. Perth 6009. (2) Children's Chest Research Centre, The New Children's Hospital, Sydney 2124. Australia.

Asthma and COPD are characterised by persistent airway inflammation. We hypothesised that this results from persistent immune stimulation by activated cells in situ in smokers who develop COPD and in asthmatics. Lymphoid aggregates (LA) have been described in asthma and COPD. We hypothesised that these structures, if present, may represent a local site within the airway for antigen presentation and T-cell stimulation to occur. We counted the number of cases of COPD and asthma of varying severity with defined lymphoid aggregates and whether they expressed HLA-OR or not. Transverse sections of 3 or 4 large airways from controls (CO), smokers with normal lung function (SC), mild (FEV1<BO\%) and severe (FEV1<20\%) COPD and mild (nonfatal NFA) and severe (fatal - FA) asthma; $n=8$ in each group, were stained with HAM56 (macrophages) and anti-HLA-DR monoclonal antibodies. HAM56 was used to determine if HLA-DR + cells within lymphoid aggregates were macrophages or not.

Figure 1

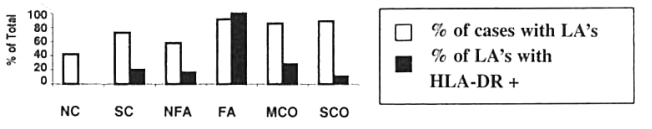

Compared with controls, the \% of cases with defined LA's was increased $(p<0.05)$ in cases of FA, mCOPD and sCOPD. The percentage of LA's staining for HLA-DR was increased $(P<0.05)$ only in cases of fatal asthma. There was little HAM56+ staining in LA's. The high percentage of cases with LA's in FA, mCOPD and sCOPD suggests that they may play a role in the development of aifflow obstruction. HLA-DR expression was increased only in FA, possibly due to an acute inflammatory stimulus. The findings suggest that lymphoid aggregates are related to severity of airflow obstruction and may provide a local site within the airway wall where antigen presentation can occur.

Support: NHMRC Australia.

Key words: Asthma, COPD, inflammation, HLA-DR

Awards: Nil

PREVALENCE OF ATOPY IN PATIENTS WITH SARCOIDOSIS.

Cornere $M^{*}$, Hopkin $R^{*}$, Douglas $D^{+}$, Wilsher $M^{*}$. Dept of Respiratory Services, Green Lane Hospital ${ }^{*}$ and Dept of Immunology, Auckland Hospital ${ }^{+}$, Auckland, New Zealand

Hypothesis: The prevalence of atopy is low in patients with sarcoidosis because their immune systems are skewed towards generating a TH1 type response.

Alm: To determine the prevalence of atopy in patients with sarcoidosis compared with normal controls.

Methods: Patients with a diagnosis of sarcoidosis not currently on treatment ( $n=98,53 F$, age range $24-75$ ) were prospectively recruited and data compared with historical controls from the European Community Health Survey (ECHS) $(n=1257,663 F$, age range 20-44). A history of asthma or allergy was determined by questionnaire derived form the ECHS. Atopy was defined as a serum specific $\operatorname{lgE}>35 \mathrm{kU} / \mathrm{L}$ to one or more of four common aeroallergens.

Pesults: Patients reported a higher prevalence of asthma ever $21.4 \%$ vs $15.9 \%$ $(p=n s)$ but lower prevalence of nasal allergy $28 \%$ vs $38 \%(p=0.06)$. The prevalence of atopy was similar for both patients and controls $31.6 \%$ vs $34.8 \%$ as was the percentage of patients with a total IgE > 100kU/L, $27.6 \%$ vs $30.5 \%$. Conclusions: Patients with sarcoidosis report similar prevalences of asthma and allergic symptoms and have similar prevalence of atopy. Further study of a subset of patients with acute or active sarcoidosis may be necessary to test the hypothesis

Supported by the Health Research Council of New Zealand 
REGULATION BY FORMOTEROL AND BUDESONIDE OF IL-8 AND STEM CELL FACTOR (SCF) LEVELS IN HUMAN CULTURED AIRWAY SMOOTH MUSCLE (ASM)

Darren Fernandes', Gary Anderson', John W Wilson², Alastair G Stewart ${ }^{1}$

(1) Department of Pharmacology University of Melbourne. Vic 3010 (2) Respiratory Medjcine, Afred Hospital, Prahran, Vic 3076

The combination of a long acting $\beta_{2}$-agonist with inhaled steroid appears to be more effective in treatment of asthma than increasing the dose of steroid. It is possible that, inter alia, long acting $\beta_{2}$-agonists have moderate antiinflammatory effects in airway disease that are complementary to or synergise with those of anti-asthma steroids. Alm: We tested the hypothesis that cytokine (IL-8 and SCF) levels in media of cultured ASM cells would be more sensitive to inhibition by the combination of $\beta_{2}$-agonist and steroid than either agent alone. Methods: ASM cells were grown to confluence, then serum-deprived for $24 \mathrm{~h}$ before treatment. Cytokine levels in smooth muscle supernatants were measured by ELISA after 0,24 , and $48 \mathrm{~h}$ incubation with IL-1 $\alpha(1 \mathrm{ng} / \mathrm{ml})$. Anti-asthma drugs were added $30 \mathrm{~min}$ prior to IL-1 $\alpha$ addition. Results: IL-8 levels increased time-dependently in culture ( $t=0 h, 5.9 \pm 1.1 \mathrm{ng} / \mathrm{mg}$ protein, $t=48 \mathrm{~h}, 24.4 \pm 10.9 \mathrm{ng} / \mathrm{mg}$ protein, $n=3$ ). $\mathrm{IL}-1 \alpha(1 \mathrm{ng} / \mathrm{ml})$ increased ASM IL-8 levels over $48 \mathrm{~h}$ (control $100 \%, \mathrm{IL}-1 \alpha$ $563 \pm 37 \%)$. Neither budesonide $(100 \mathrm{nM})$ nor formoterol $(10 \mathrm{nM})$ affected basal IL-8 levels. Budesonide ( $1-100 \mathrm{nM}$ ) concentration-dependently reduced IL-1 $\alpha$-stimulated IL-8 release (IL-1 $\alpha$ 100\%; IL-1 $\alpha /$ bud $1 \mathrm{nM} 57 \pm 8 \%$; $(L-1 \alpha /$ bud $100 \mathrm{nM} 49 \pm 8 \%$ ). However, formoterol ( $10 \mathrm{nM}$ ) had no inhibitory effect, either alone or when co-incubated with budesonide. SCF levels also increased time-dependently in culture ( $t=0 \mathrm{~h}, 113 \pm 38 \mathrm{pg} / \mathrm{mg}$ protein; $t=48 \mathrm{~h}$ $448 \pm 142 \mathrm{pg} / \mathrm{mg}$ protein). In contrast to the stimulatory effect of $(\mathrm{L}-\alpha$ on IL-B levels, IL-1 $\alpha$ reduced SCF levels over $48 \mathrm{~h}$ (control 100\%; IL-1 $1 \alpha 72 \pm 10 \%$ ). Budesonide further reduced SCF release $(53 \pm 5 \%$ control), while formoterol produced no further inhibitory effect, alone or in combination with budesonide. Conclusions: These results suggest that ASM levels of IL-8 and SCF were reduced by budesonide alone and this action was not influenced by the long-acting $\beta_{2}$-agonist formoterol.

Supported by Astra (Sweden)

Key words: Airway smooth muscle, formoterol, budesonide, IL-8, stem cell factor Nominatlon for awards: Nil

\section{P75}

VISUALISATION OF ZINC (Zn) IN GRANULES OF MAST CELLS AND ITS ROLE IN SUPPRESSION OF NF-KB IN AIRWAY EPITHELIAL CELLS

Lien Ho', Richard Ruffin and Peter Zalewski. Department of Medicine. The University of Adelaide. The Queen Elizabeth Hospital. Woodville Rd. Woodville. SA. 5011

The human respiratory tract is pich in mast cells, especially beneath the bronchial epithelium and these play an important role in airway inflammation. Zine is known to be anti-inflammatory and one of our interests is to determine whether mast cells are rich in $\mathrm{Zn}$, which is released during degranulation. We are also interested in whether $\mathrm{Zn}$ has an antiinflammatory effect through the suppression of NF- $\mathrm{KB}$ transcription factor. The distribution of intracellular labile $\mathrm{Zn}$ was visualised in cord blood and HMC-1 mast cells using a Zn-specific fluorophore, Zinquin and image analysis. High levels of Zinquin fluorescence indicated the cells were rich in $\mathrm{Zn}$. Mast cells were degranulated with IgE/anti-IgE, PMA, and $\mathrm{Ca}$ ionophore A23187 and compound $48 / 80$ for $4 \mathrm{hr}$ at $37^{\circ} \mathrm{C}$. Zinquin fluorescence was decreased in HMC-1 treated with IgE/anti-IgE from $101.5 \pm 2.6$ to $59.3 \pm 3.7$ pixels $(p<0.001)$. NF-xB was activated in NCl-H292 bronchial epithelial cells with $20 \mathrm{ng} / \mathrm{ml}$ TNF- $\alpha$ for $4 \mathrm{hr}$ at $37^{\circ} \mathrm{C}$. It was detected by immunofluorescent labelling using a primary antibody specific to the p65 subunit of NF- $\mathrm{xB}$. Zn supplementation by $25 \mu \mathrm{M} \mathrm{ZnSO} \mathrm{Z}_{\mathrm{A}}+1 \mu \mathrm{M}$ pyrithione resulted in suppression of NF-KB. Differences in levels of fluorescence were seen in untreated cells $(51.6 \pm 1.5), T N F-\alpha$ treated $(111.1 \pm 2.1)$ and $\mathrm{Zn}+\mathrm{TNF}-\alpha$ treated cells $(57.7$ \pm 1.3 ) pixels $(p<0.001)$. These results indicate that $Z n$ is likely to be released from mast cells and have a role in the suppression of NF-kB in airway opithelial cells, with possible implications for asthma.

Supported by the University of Adelaide Scholarship Key words: Mast cells, zinc, degranulation, inflammation and NF-kB.
AN INVESTIGATION OF HOUSEKEEPING GENE UTIILITY FOR NORMALIZATION OF GENE EXPRESSION IN HUMAN CULTURED AIRWAY SMOOTH MUSCLE

E. Guida \& A.G. Stewart.

Department of Pharmacology. The University of Melbourne. Victoria 3010. Interest is growing in the regulation of gene expression in human cultured airway smooth muscle (HASM) in relation to cell cycle, phenotype and cytokine production. The normalization of gene expression by housekeeping genes uses the assumption that these housekeeping genes, such as tubulin or $23 \mathrm{kD}$ Highly Basic Protein (23 kD HBP), or more commonly, $\beta$-actin or glyceraldehyde 3- phosphate dehydrogenase (GAPDH), are expressed at constant levels. Alm: Our aim was to identify the gene which best meets the criteria for housekeeping: namely, that its level of expression be unaffected by different treatment regimens and that the variance of both its absolute levels and the variance of the normalized product of interest are minimised. Methods: We have used CDNA arrays (Atlas ${ }^{T M}$ array) and northern analysis to pattern gene expression in mitogen-stimulated HASM in the presence and absence of anti-asthma agents. Results: The presence of multiple housekeeping genes in the array revealed that different mitogenic and drug treatments altered expression of certain housekeeping genes. These observations were confirmed by northern Analysis, a more quantitatively reliable method than the array methodology. B-Actin was up regulated by thrombin ( $150 \%$ compared to control), conversely tubulin was down regulated by 2 -methoxyestradiol ( $50 \%$ compared to control). Given that there was a significant difference in variance of the absolute levels of these two housekeeping genes across the different treatment regimens, these genes were not further evaluated. Although there was no significant difference in the variance of the absolute levels of either GAPDH or $23 \mathrm{kD}$ $\mathrm{HBP}$, the $23 \mathrm{KD}$ HBP housekeeping gene had a significantly higher coefficient of variation (paired $t$-test, $p<0.0001$ ) when used to normalized either cyclin D1 or $\mathrm{P}_{21}{ }^{\mathrm{ClP} 1}$ genes. Conclusion: The present study has shown that, in HASM, of the four housekeeping genes examined, GAPDH is the most reliable housekeeping gene when normalizing gene expression levels.

Supported by Glaxo Wellcome (UK), Amrad Operations Pty Ltd, NHMRC (Australia)

Key words: Housekeeping gene, human aiway smooth muscle Nominations for Awards: Nil

\section{FLOW CYTOMETRIC ANALYSIS OF BRONCHIAL LAVAGE}

S.Hodge',2,G. Hodge ${ }^{3}$, P.Han ${ }^{3}$, M.Holmes' 1 , R.Scicchitano

1 Thoracic Medicine, Royal Adelaide Hospital

2 Department of Medicine, University of Adelaide

3 Haematology Department, Women's and Children's Hospital, North Adelaide

The characterisation of cellular infiltrates in bronchial lavage (BAL) is important in the diagnosis of a variety of lung disorders (Jeffery, 1998). In chronic bronchitis, the predominant cell infiltrate in BAL is macrophages and $\mathrm{T}$ cells. In chronic obstructive pulmonary disease (COPD), macrophages, neutrophils and CD8 positive T cells are present. Asthrna is characterised by a marked eosinophil infiltrate and CD4 positive T cells. We describe a rapid multiparameter flow cytometric assay using fluorescently conjugated monoclonal antibodies to characterise cellular subtypes in BAL.

The use of standard control beads allows simultaneous analysis of absolute numbers of cells using a single platform assay, without the need for manual cell counts. There was good agreement between this automated counting method (counting 100,000 cel/s) and standard manual counting methods (counting 200 cells) to quantify absolute cell numbers. We conclude that the speed and accuracy of defining cell populations in BAL using flow cytometric techniques may aid in the differential diagnosis of lung disease. This technique would also be amenable to analysis of cell types in samples of sputum and bronchial brushing.

\section{Reference:}

Jeffery, $P$. (1998) Structural and inflammatory changes in COPD: a comparison with asthma. Thorax; 53: 129-136. 
IL-4 AND TNF- $\alpha$ INHIBIT TGF- $\beta$ PRODUCTION BY HUMAN LUNG EPITHELIAL CELL LINES: RELEVANCE TO COPD

S. Hodge 1.2,G. Hodge ${ }^{3}$, R.Flower4, M. Holmes' ${ }^{1}$ R.Scicchitano1

1 Thoracic Medicine, Royal Adelaide Hospital

2 Department of Medicine, University of Adelaide

3 Haematology Department, Women's and Children's Hospital, North

Adelaide

4 PALMS, Royal North Shore Hospital, Sydney.

Human bronchial epithelial cells are known to secrete an array of inflammatory cytokines which play a role in immune responses in COPD. However, the regulatory mechanisms governing cytokine production in bronchial epithelia are largely unknown. TNF- $\alpha$ is a pro-inflammatory cytokine, known to be upregulated in COPD. IL-4 has been reported to induce IL-8 release by bronchial epithelial cells and to inhibit fibronectin release by these cells. TGF- $\beta$ is an immunosuppressive cytokine, produced by many cells including airway epithelial cells and is involved in airway repair and fibronectin synthesis.

We studied the cytokine interactions that might regulate TGF- $\beta$ production using two epithelial cell lines (A549 and 16HBE). Cells were stimulated with various combinations of TNF- $\alpha$ and IL-4 $(20 \mathrm{ng} / \mathrm{ml})$ for $24 \mathrm{~h}$. TGFB and IL-4 production was measured by flow cytometry and immunohistochemical techniques. TNF- $\alpha$ significantly upregulated production of IL-4 from cultured epithelial cells. Unstimulated cells spontaneously released TGF- $\beta$. The production of TGF- $\beta$ was signiticantly increased by PMA. TNF- $\alpha$ and IL-4 inhibited production of TGF- $\beta$ by both epithelial cell lines. The inhibitory effect of TNF- $\alpha$ and IL- 4 on TGF- $\beta$ synthesis was additive. We conclude from our study that TNF- $\alpha$ induces IL-4 release from human epithelial cells and the inhibitory effect of IL- 4 and TNF- $\alpha$ on TGF- $\beta$ synthesis by these cells was additive. The inhibitory effect of IL- 4 and TNF- $\alpha$ on the regulatory cytokine TGF- $\beta$ in the bronchial mucosa, may contribute to the progression of the inflammatory response and decrease in repair processes in COPD.

\section{ANT JO 1 ANTIBODY AND LUNG DISEASE}

\section{T J McWilliams and M L Wilsher}

Respiratory Services, Green Lane Hospital, Auckland. New Zęaland Introduction: The anti Jo 1 antibody is known to be associated with adult dermatomyositis and polymyositis. A much smaller group of these patients present with lung disease. We reviewed this group of patients at our hospital. Methods: A retrospective case review of patients with documented anti Jo1 antibody and lung disease. Demographic information, age at presentation, diagnostic investigations, treatment and progress were documented. Results: 4 female patients age 40-47 presented with respiratory symptoms. Three required open lung biopsies for diagnosis; two had organizing pneumonitis and one follicular bronchiolitis. One patient has an accepted high resolution CT diagnosis of organizing pneumonitis. Three of the patients developed connective tissue diseases after the onset of respiratory disease (dermatomyositis (1), polymyositis (1) and one rheumatoid disease and one has no clinical manifestation of a connective tissue disease. Two patients developed respiratory disease before becoming positive for anti Jo 1 antibodies. All patients required treatment with corticosteroids and three were also treated with steroid sparing agents. Response was poor in alf. Conclusion: This review highlights this small but important group of patients with anti Jo 1 antibody and lung disease. In this group symptoms of connective tissue disorders may develop after the onset of respiratory disease. These patients often respond poorly to treatment.

\section{IDENTIFICATION OF Th1 RESPONSE TO NON-TYYPEABLE} HAEMOPHILUS INFLUENZA BY FLOW CYTOMETRY

Paul King 1.4. Paul Hutchinson'2, Paul Johnson ${ }^{3}$, Peter Holmes 1,4, Nicholas Freezer 1.4 , Stephen Holdsworth2.

Department of Respiratory Medicine. (2) Department of Medicine/Clinical Immunology, (3) Department of Infectious Disease \& Clinical Epidemiology Monash Medical Centre and (4) Monash University Centre for Heart and Chest Research, Clayton 3168. Victoria

Most adults are colonised with Haemophilus influenza, which is one of the major causes of respiratory infection. The role of the T lymphocyte and in particular the T-helper (Th or CD4) cell, in the immune response to haemophilus infection has not been established. Th cell function can be assessed by the measurement of Th1 (IFN- $\gamma$ ) or Th2 (IL-4, IL-5) cytokines that are produced following antigen exposure. Aims: To measure whether healthy adults produce a Th1 or Th2 response when exposed to non-typeable haemophilus influenza (NTHi) in vitro, using flow cytometry to measure intracellular cytokine production. Methods: Flow cytometry has been described to measure intracellular cytokine production to cytomegalovirus (CMV) infection. This technique is performed by adding antigen and costimulatory antibody (CD28 and CD49) to whole blood, which is incubated for 6 hours. Red blood cells were lysed and leucocytes fixed, permeabilised and stained with immunoflourescent antibodies for CD4, CD69, IFN- $\gamma, \mathrm{IL}-2, \mathrm{IL}-4$, and IL-5. Binding of the antibodies to the cytokines is then measured using the flow cytometer. We validated this technique by screening 10 subject's response to CMV antigen; 5 were responders with $0.63 \% \pm 0.28$ of Th cells producing IFN- $\gamma_{1}$ consistent with a Th1 response. NTHi obtained from a child with conjunctivitis, was cultured on agar plates, washed and suspended at a concentration of $2.0 \mathrm{McFarlane}$ units, heat inactivated and then disrupted by ultrasound sonication. $2 \mathrm{ml}$ of biood was taken from 4 healthy male subjects. $1 \mathrm{ml}$ of blood from each subject was exposed to $50 \mu \mathrm{l}$ of NTHi and the other $\mathrm{ml}$ served-as a control. The response to NTHi compared with control was measured using flow cytometry. Results: Three of the six subjects produced a distinct Th1 response with $0.17 \%$ of Th cells producing IFN- $\gamma$, compared with control of $0.00 \%$, with undetectable levels of IL-4 and IL-5. This result was comparable to that produced from the CMV group. This response was prevented by the addition of MHC-2 blocking antibody contirming the role of the Th cell. Conclusion: The results show that a Th1 response to NTHi can be measured by flow cytometry.

Key words: Haemophilus, Th1/Th2 cytokine response, flow cytometer

DEVELOPMENT OF A NEW ASSAY FOR MEASUREMENT OF INFLAMMATION-RELATED PHOSPHOLIPASE A ACTIVITY IN HUMAN PLASMA

Nenad Petrovic, Carolyn Grove, and Philip Thompson

Department of Medicine. University of Western Australia, and Asthma and Allergy Research institute, Perth, Western Australia

Phospholipases $\mathrm{A}_{2}(\mathrm{PLA}$ ) play crucial roles in diverse cellular responses, including phospholipid digestion and metabolism, host defense and signal transduction and providing precursors for eicosanoid generdtion. Mammalian tissues and cells generally contain more than one PLA enzyme, each of which is regulated independently and has distinct functions. Secreted PLA $\left(s P L A_{2}\right)$ found in plasma has been linked with inflammatory processes. One of the reported characteristics of the SPLA $A_{2}$ is its ability to bind to heparin. Using specifically designed heparin affinity chromatography, $\mathbf{S P L A} \mathrm{A}_{2}$ was partially purified from human plasma samples. The resulting enzyme preparation was assayed with a newly developed activity assay using a chromogenic lipid substrate 4-nitro-3-(octanoyloxy)-benzoic acid. The products of the $S P L A_{2}$ reaction were detected spectrophotometrically in a microplate assay. In optimized conditions the plasma $S P L A_{2}$ reaction followed Michaelis-Menten kinetics. Mean of SPLA $A_{2}$ activities measured in plasma of 23 control subjects was $421.4 \pm 29.4$ units [nmoles of product formed in $1 \mathrm{~h}$ per milligram of protein bound to the heparin column]. The amount of plasma needed for $s P L A_{2}$ activity assay is relatively small $(0.5 \mathrm{ml})$ and the assay is rapid, simple and very reproducible (mean of standard deviation observed in measurements was $4.95 \%$ ). The assay was successfully used to analyse $\mathrm{SP} L \mathrm{~A}_{2}$ activity in plasma from a cohort of 64 stable asthmatics ( 43 mild and 21 severe) and revealed a mean activity of $420.4 \pm 18.4$ and $449.0 \pm 28.2$ units respectively.

Supported by Asthma Foundation of Western Australia

Key words: PLA $A_{2}$, Asthma, plasma, assay.

NomInatlons for awards: Nil 
IMPORTANCE OF GROWTH AND STRESS-RELATED SIGNALLING PATHWAYS IN HUMAN AIRWAY SMOOTH MUSCLE CELL PROLIFERATION

CE Ravenhall \& AG Stewart.

Department of Pharmacology, University of Melbourne, Victoria 3010.

Hyperplasia and hypertrophy of aiway smooth muscle contributes to airway wall thickening and hyperresponsiveness in asthma. Understanding the intracellular signalling of airway smooth muscle proliferation may provide new anti-asthma drug targets. Aim: in this study, the relative importance of the activity of the two distinct families of MAPKs (extracellular signalregulated kinase (ERK) and p38HOG) for the regulation of cyclin D1 levels and DNA synthesis was investigated using the inhibitors PD 98059 (ERK) and SB 203580 (p38HOG). Methods: Cyclin D1 protein and mRNA levels were examined by western and northern blotting, respectively. ERK phosphorylation was measured by western blotting, while kinase activity was measured following ERK immunoprecipitation. DNA synthesis was assessed by the incorporation of [ $\left.{ }^{3 \mathrm{H}}\right]$-thymidine into newly synthesised DNA. Results: Both thrombin $(0.3$ and $3 \mathrm{U} / \mathrm{ml})$ and bFGF $(0.3$ and $3 \mathrm{nM})$ increased ERK phosphorylation and activity levels, cyclin D1 levels at $20 \mathrm{~h}$ and DNA synthesis between 24 and $28 \mathrm{~h}$ after mitogen addition. Although PD $98059(30 \mu \mathrm{M})$ reduced ERK activity to baseline levels, it had no effect on cyclin D1 mRNA levels and reduced the increase in cyclin D1 protein levels in response to only the lower mitogen concentrations. PD 98059 completely prevented thrombin-stimulated DNA synthesis, whereas DNA synthesis in response to $3 \mathrm{nM}$ DFGF was only partially inhibited (0.3 nM: 81 $\pm 11 \%$; 3 nM: $67 \pm 10 \%$ inhibition). Conversely, SB $203580(10 \mu \mathrm{M})$ inhibited bFGF-stimulated DNA synthesis ( $0.3 \mathrm{nM}: 56 \pm 3.2 ; 3 \mathrm{nM}: 92 \pm$ $9.7 \%$ inhibition), but had no significant effect on thrombin-stimulated DNA synthesis. SB 203580 did not reduce mitogen-stimulated cyclin D1 mRNA or protein levels. Conclusions: ${ }^{3} 8^{\mathrm{HOG}}$ contributes to the signalling of bFGF, but not thrombin-stimulated DNA synthesis through activity unrelated to the regulation of cyclin D1 levels.

Supported by Glaxo-Wellcome (UK) \& NHMRC (Australia).

Key words: airway smooth muscle, signal transduction, extracellular signalregulated protein kinase, p38HOG.

ONCOSTATIN M (OSM) AND LEUKEMIA INHIBITORY FACTOR (LIF) INDUCE FIBROBLAST PROLIFERATION: MODULATION BY THE CYCLOOXYGENASE PATHWAY.

Amelia Scaffidi1,2, Philip Thompson 1,2, Darryl Knight1,2.

(1) Asthma \& Allergy Research Institute, (2) Department of Medicine. University of Western Australia, Nediands. Western Australia.

Introductlon: OSM and LIF are members of the IL-6 family of cytokines and influence the behaviour of a variety of cell types. OSM has been shown to upregulate the production of epithelial anti-proteases and fibroblast-derived tissue inhibitor of metalloprotease and also induces the release of collagen from fibroblasts. We have recently demonstrated the widespread distribution of LIF mRNA within the lung and its release from human lung fibroblast cultures (HFL-1). Hypothesis: We hypothesised that OSM and LIF may play a role in airway remodelling by regulating fibroblast proliferation. Methods: We examined the proliferative effects of stimulating HFL-1 cultures with OSM $(0.2,2$ and $20 \mathrm{ng} / \mathrm{ml})$ or LIF $(0.5$ and $50 \mathrm{ng} / \mathrm{ml})$. Proliferation was assessed via a MTS assay and direct cell counts at 24,48 and $72 \mathrm{hr}$. Results: Both OSM and LIF enhanced the mitotic activity of HFL-1 in a time and dose dependent manner. Maximal proliferation in response to OSM was $42 \%$ above control and observed after $72 \mathrm{hrs}$, at a concentration of $2 \mathrm{ng} / \mathrm{ml}$. Incubation with the cyclooxygenase 2 (COX-2) inhibitor nimuselide $(2 \mu \mathrm{M})$ or the MAPK inhibitor PD98059 $(10 \mu \mathrm{M})$ enhanced the mitogenic effect of OSM above control levels to $59 \%$ and $71 \%$ respectively. In comparison, LIF exerted its maximal effect at $48 \mathrm{hr}$ at a concentration of $50 \mathrm{ng} / \mathrm{ml}$, reaching an $80 \%$ increase in proliferation above control, which declined to $25 \%$ by 72 hr. Conclusion: These results demonstrate that OSM and LIF induce fibroblast proliferation and that for OSM at least, COX-2 release as well as signalling via the MAPK pathway may act to modify this mitogenic effect. These data support the hypothesis that OSM and LIF contribute to airway remodelling and fibrosis.

Supported by the NHMRC and the Raine Medical Foundation. Key words: OSM, LIF, COX-2, fibroblasts, proliferation, asthma.

\section{IRON OVERLOAD AND NO-DERIVED OXIDATIVE STRESS FOLLOWING} LUNG TRANSPLANTATION.

Reid D MRCP., Snell GI FRACP., Ward R M Phil., Krishnaswamy R MSc., Zheng L MD., Ward C M Phil., Williams TJ FRACP., Walters EH DM, FRACP. Department of Respiratory Medicine, Alfred Hospital and Monash University Medical School, Melbourne, Victoria.

Background: The local generation of harmful reactive oxygen species (ROS) may contribute to the development of chronic rejection and irreversible airflow limitation (bronchiolitis obliterans syndrome - BOS) following lung transplantation (LT). Hypothesis: Chemically active iron and nitric oxide (NO)-derived radicals within the allograft may add to the oxidative burden. Methods: As a marker of potential iron load we determined the concentration of ferritin in bronchoalveolar lavage fluid (BALF) and assessed the relationship to haemosiderin-laden macrophages (HLM) in 14 stable LT recipients (sLTR) and 7 subjects with BOS. HLM were quantified using a haemosiderin-score (HS). BALF nitrite and albumin concentrations were also determined as markers of nitric oxide-derived oxidative stress and microvascular leakage. Results: BALF ferritin levels and HS were significantly higher in LTR than controls $(p<0.01)$, but there was no difference between LT groups. There was a significant relationship between ferritin concentration and HS in LTR ( $r=0.7, p<0.01)$. BALF nitrite was significantly elevated in LTR compared to controls $(p<0.01)$. There was a significant relationship between BALF nitrite and \%neutrophils $(r=0.4$, $p<0.01)$, particularly in BOS $(r=0.9, p<0.01)$ but only a weak relationship to BALF ferritin $(r=0.3, p=0.2)$ and no relationship to BALF albumin. In BOS patients there was a trend toward higher BALF albumin levels compared with controls $(p=0.07)$ and a significant relationship to BALF ferritin $(r=0.8$, $\mathrm{p}=0.05$ ) but no such relationship in sLTR. Conclusions: Our findings suggest the lung allograft could be subject to significant iron-generated oxidative stress over time which may be exacerbated by NO and neutrophilderived ROS. Microvascular leakage and plasma exudation may be an independent feature of established chronic rejection which potentiates the iron over-load and contributes to further aiway damage and remodelling.

IDENTIFICATION OF THE BRAINSTEM REGIONS INVOLVED WITH COUGHING : A STUDY USING C-FOS IMMUNOHISTOCHEMISTRY

Puntarica Suwanprathes ${ }^{1,2}$, Glenn Hunt ${ }^{3}$, Antony Breslin ${ }^{1}$, Alvin Ing $^{1}$ and Meng Ngu².

(1) Respiratory (2) Gastroenterology Units and (3) Psychological Medicine. Concord Hospital. The University of Sydney, NSW AUSTRALIA

The central control of cough is thought to arise from an ill defined region within the brainstem. The aim of this study was to identify, using c-fos immunohistochemistry, afferent pathways which are activated by mechanical stimulation of the larynx and trachea. Methods: In pentobarbital anaesthetised Wistar rats $(n=7)$, the trachea was exposed and a small incision was made to insert a fine plastic tube to mechanically stimulate the larynx and trachea for 30 minutes. Rats who were similarly anaesthetised and whose trachea was cut but no tube inserted, acted as a control group $(n=4)$. Rats remained anaesthetised for a further one hour before they were perfused and their brains processed for $c$-fos immunohistochemistry. Results: c-Fos immunoreactivity was increased in a number of brain regions in the rats receiving mechanical stimulation ( $p<0.05$, one-way ANOVA). These areas included the Kolliker-Fuse nucleus, the medial parabrachial nucleus (external part) and the nucleus of the solitary tract (nTS) (medial and ventrolateral parts) which are involved with respiratory and autonomic functions. Conclusions: The pattern of $c$-fos expression in the mechanically stimulated rats was similar to a previous study (Gestreau et al, J Neurosci $1997 ; 17: 9340-9352)$ in which coughing was induced by electrical stimulation of the superior laryngeal nerve in cats. Our data suggest that stimulation of mechanoreceptors in the larynx and trachea activates neuronal pathways which project to nTS (medial and Ventrolateral parts) in the medulia.

Key words : trachea, laryn $x$, cough, $c$-fos, the solitary tract, rat Nominations for Award : nil 
A NOVEL TECHNIQUE: PRIMARY FIBROBLAST CULTURES FROM TRANSBRONCHIAL BIOPSIES OF LUNG TRANSPLANT RECIPIENTS

\section{Tamm12, M. Roth1.2, M. Malouf11, P. Chhajed1, P. Johnson 2 J. Black2,} A. Glanville ${ }^{1}$.

Heart Lung Transplant Unit1, St. Vincents Hospital and Department of Pharmacology2, University of Sydney, Sydney, Australia Longterm survival following lung transplantation (LT) is limited by the development of bronchiolitis obliterans (BO). Subepithelial fibrosis may be found in transbronchial biopsies (TBB) prior to the diagnosis of $\mathrm{BO}$. In order to study the pathogenesis of $\mathrm{BO}$ we attempted to establish for the first time primary human fibroblast cell cultures from TBBs of LT recipients. One to two TBB samples of LT patients undergoing diagnostic or surveillance TBB were collected in sterile PBS supplemented with antibiotics. Biopsies were cut in 4 to 8 small pieces and placed onto 12 well culture plates or $25 \mathrm{~cm}^{2}$ culture flasks and cultured under routine conditions $\left(21 \% \mathrm{O}_{2}, 5 \% \mathrm{CO}_{21}\right.$ $37^{\circ} \mathrm{C}$ ). Culture medium consisted of RPMI $1640,10 \% \mathrm{FCS}$, L-glutamine and HEPES. Fibroblast culture results of 50 consecutive TBBs were analysed. The 50 TBBs were performed in $30 \mathrm{LT}$ recipients $13 \mathrm{CF}, 8$ emphysema, 4 $\mathrm{PPH}, 4$ CFA, 1 VSD) who underwent bilateral (19), single (8) or heart-lung (3) transplantation. The indication for TBB were symptoms, a drop in FEV and/or infiltrates on chest $X$ ray in 27 and a surveillance or follow up procedure in 23 cases. The overall culture success rate was $54 \%(27 / 50)$ defined as cells reaching confluence to be further passaged. The culture success was independent of the age, the transplant procedure, the underlying lung disease, the indication for TBB (symptomatic/surveillance/ follow up), the result of histology in regard to rejection $\left(A_{0} / A_{1}\right.$ versus $A_{2} / A_{3}$ i $B_{0} / B_{1}$ versus $\left.B_{2} / B_{3}\right)$ and the $B O S$ stage. Bacterial/fungal colonisation of the bronchial tree was frequent but showed no negative influence on cell culture results. Fibroblasts could be cultured from $48 \%(11 / 23)$ of samples with a clinical diagnosis of pulmonary infection (BALTBB result) and from $52 \%$ (12/23) TBBs of patients without infection.

Summary and conclusion: we have established a novel method of culturing primary human lung fibroblasts from lung transplant recipients. These cultures provide a unique in vitro model of human tissue to study pathogenetic mechanisms of bronchiolitis obliterans at an early stage.

\section{P87}

WEGENER'S GRANULOMATOSIS DIAGNOSED IN PREGNANCY

Alistair Wright'1, Peter Holmes', Steven Holdsworth².

1. Department of Respiratory Medicine, Monash Medical Centre. 2. Department of Medicine. Monash University. Clayton Rd, Clayton, Victoria, Australia. 3168

CASE: A 31 year old woman presented at 9 weeks gestation. Her past history included an episode of pericarditis 5 years previously and a 2 year history of sinusitis and nasal congestion which had required surgery. At presentation the patient had a 4 week history of night sweats and an asymmetric polyarthritis and a 1 week history of haemoptysis, dysphonia and intermittent epistaxis. Examination revealed a low grade fever and a number of tender, mildly swollen joints. Her nasal mucosa was erythematous and swollen. INVESTIGATIONS: A CXR revealed bilateral pulmonary infiltrates. The cytoplasmic anti-neutrophil cytoplasmic antibody (c-ANCA) was moderately elevated (titre $=124$ ANCA units, normal $=$ undetectable) and the ESR was $106 \mathrm{~mm} / \mathrm{hr}$. Renal function was normal. A biopsy of her nasal mucosa revealed only non-specific inflammation. DIAGNOSIS: A diagnosis of Wegener's granulomatosis was made and pulse methylprednisolone was given. There was a prompt improvement in the patient's clinical condition with resolution of her haemoptysis and improvement in her voice and joint symptoms and normalisation of her inflammatory markers including the c-ANCA. ISSUES: The patient wished to continue her pregnancy. In view of (a) the possiple teratogenic effects of cyclophosphamide given in the first trimester of pregnancy and (b) of her prompt response to steroids and (c) the limited nature of her disease it was decided to withhold cyclophosphamide and to treat her with prednisolone and close observation. PROGRESS: The patient's health continued without complication until 34 weeks gestation when she represented with haemoptysis. She was treated with further pulse methylprednisolone and the child was delivered. Following delivery cyclophosphamide was commenced. Both the mother and baby are currently well. SUMMARY: A literature search confirms this to be the first case of Wegener's granulomatosis diagnosed during the $1^{\text {st }}$ trimester of pregnancy where there has been a successful maternal and foetal outcome.

Key words: Wegener's granulamatosis, pregnancy, prednisolone.
EXPRESSION OF CYCLOOXYGENASE IN HUMAN DENDRITIC CELLS. John Upham, Darryl Knight \& Philip Thompson.

\section{Asthma \& Allergy Research Institute, and University of Western Australia} Dept. of Medicine. Sir Charles Gairdner Hospital. Nedlands WA 6009.

It has been proposed that dendritic cells (DC) play a key role in regulating the balance between Th1 and Th2 immunity, possibly through release of prostaglandins (PGs), and cytokines such as IL-12 and IL-10 Thus, understanding the regulation of cyclooxygenase (COX) expression and PG synthesis in DC may shed important light on the induction \& regulation of allergic disease. As little is known about the regulation of $\mathrm{COX}-1$ and $\mathrm{COX}-2$ in human dendritic cells (DC), the expression of these enzymes in DC and monocytes was assessed by immunocytochemical staining and by intracellular flow cytometry of permeabilised cells. COX-2 staining was detected at low intensity in only a small minority of freshly isolated blood DC and monocytes, but was dramatically upregulated following exposure to LPS (10 $\mathrm{ng} / \mathrm{ml})$. Intensification of staining was apparent by $4 \mathrm{~h}$, and reached maximal leveis within $24 \mathrm{~h}$, with $80-90 \%$ of cells showing COX-2 expression. Exposure of freshly isolated blood DC and monocytes to GM-CSF \& IL-4 for $24 \mathrm{~h}$ did not alter COX-2 expression, whereas monocyte-derived DC (obtained by culturing monocytes with GM-CSF \& IL-4 for 7 days) exhibited intense COX-2 staining which was further enhanced by exposure to LPS. In contrast, COX-1 staining was detected at low-moderate intensity in the DC \& monocyte populations studied, and its expression was not appreciably altered by LPS. These findings indicate that both COX-1 and COX-2 can be detected in human DC. The expression of COX-2 in DC varies in response to inflammatory stimuli and/or cellular maturation, and the influence of other cytokines and tissue derived factors needs to be explored further.

\section{Supported by the NHMRC}

Key words: dendritic cells, prosiaglandins, cyclooxygenase

Posters - COPD

OUTCOMES AND COSTS OF AN OUT-PATIENT EXERCISE PROGRAM FOR INDIVIDUALS WITH CHRONIC LUNG DISEASE

Jenkins SC1, 2, Cecins NM' \& Collins GB2

(1) Physiotherapy Department. Sir Charles Gairdner Hospital, Perth 6009 and (2) School of Physiotherapy. Curtin University of Technology. Perth. Western Australia 6008

Exercise training is an established component of the management of patients with chronic lung disease, however there is little data regarding the cost of such programs. Aim: To determine the outcomes and costs of an out-patient exercise program. Methods: 119 patients were referred during 1998. Eighty-five patients were assessed of whom $72(83 \%$ with chronic obstructive lung disease) entered an 8 week exercise program. This was a continuous rolling program and patients attended twice per week. Classes were supervised by one physiotherapist with an average of 8 patients per class. Exercise consisted of a $20-30 \mathrm{~min}$ walk at $60-85 \%$ estimated peak oxygen consumption followed by a $30 \mathrm{~min}$ exercise circuit. Outcomes included exercise capacity (field walking tests) and quality of life (QOL [SF36 and Chronic Respiratory Disease Questionnaire]). Also provided was a once per week maintenance exercise class for patients with end-stage lung disease who had completed the 8 week program. For costing purposes seven categories of service were considered including the 8 week program, the maintenance program and a program for lung volume reduction surgery (LVRS) patients. Analyses were based on 1998 physiotherapy salaries and the costs of consumables only. Results: Fifty-seven patients (36 males) completed the program (20.8\% attrition). Mean (SD) age and FEV1 were $65.5(9.3)$ years and $41.4(23.7) \%$ predicted. Significant improvements in exercise capacity and QOL. were demonstrated following the 8 week program $(p<0.05)$. The cost of this program was $\$ 249$ per patient. The respective costs for the maintenance and LVRS programs were $\$ 427$ and \$766. Conclusions: Significant benefits in exercise capacity and $\mathrm{QOL}$ can be demonstrated from an out-patient exercise program and the cost of this type of program is modest.

Key words: Exercise training, pulmonary rehabilitation, costs, outcomes 
PATIENT CHANGES IN HEALTH LOCUS OF CONTROL FOLLOWING PULMONARY REHABILITATION

Paul Cafarella, Pieter Walker and Peter Frith

Repatriation General Hospital, Daws Rd, Daw Park, South Australia, 5041

There are psychoiogical benefits from Pulmonary Rehabilitation (PR) (Mahler, 1998). PR should improve perception of control over one's health, which in turn should benefit health related behaviour. Some studies have considered the influence of PR on self efficacy, but none has assessed the effects of PR on health related perceptions of locus of control. Aim: To evaluate effects of PR on psychological morbidity and locus of control. Method: In a controlled design, 100 patients with COPD completed the condition specific version of the Multidimensional Health Locus of Control scale (MHLC), General Health Questionnaire (GHQ) and the COPD Self Efficacy scale. Results: T-tests for paired samples indicate that patients had a more internal health locus of control (IHLC) following PR (pre PR mean $=29.55, s d=8.50$ vs post $P R$ mean $=34.87, s d=8.01, p<.001$ ) and were less chance oriented (CHLC) (pre $P R$ mean=24.16, sd=7.65 vs post $P R$ mean=22.01, $s d=7.17, p<.05$ ). However, there was no significant difference in the likelihood of patients placing their health locus of control in the hands of powerful others (health professionals) (PHLC) (pre PR mean $=38.24$, $s d=6.06$ vs post $P R$ mean $=38.41, s d=5.61, p>0.05$ ). Patients had significantly lower rates of psychological morbidity following PR (pre PR mean $=6.42$, $s d=6.84$ vs post $P R$ mean $=4.01, s d=5.49, p<0.00$ ).

Conclusions: The results imply that PR helps respiratory patients recognise that self-responsibility for actions influences their clinical state. They are less likely to leave symptoms to fate and more inclined to self monitor. PR appears to foster patient empowerment and self care.

Key words: COPD, pulmonary rehabilitation, health locus of control, selfefficacy

\section{EVALUATION OF AN OUTPATIENT PULMONARY REHABILITATION} PROGRAMME

Vanessa McDonald, Kim Wild, Kim Cookson, Louise Dymond, Peter G Gibson

Department of Respiratory and Sleep Medicine. John Hunter Hospital. Newcastle

Pulmonary Rehabilitation is an important part of COPD management. In 1994 a Pulmonary Rehabilitation Programme (PRP) was developed to reduce dyspnoea, panic and anxiety and improve exercise tolerance, confidence, disease management skills, knowledge and overall quality of life in COPD. The aim of this study was to evaluate the effect of the PRP on exercise tolerance, quality of life, and readmission for people with COPD. Design: Cross-sectional analytic survey. Methods: PRP records were audited over a 54 month period. A 6 minute walk test with dyspnoea rating (BORG scale) and quality of life assessment (using CRDQ) were conducted prior to and at completion of our 7 week PRP. A hospital records search for COPD admissions (DRG177) between $1994-1998$ was conducted and readmissions compared between those who were referred and completed the PRP, and those who were referred but did not complete. Results: There were 316 referrals to the PRP of whom $172(54 \%)$ completed the PRP. Exercise tolerance, perception of dyspnoea, and quality of life all improved significartly following participation in the programme. The 6 minute walk distance, mean(sd) improved by 65(137) metres from 283(157) $\mathrm{m}$ to $348(112) \mathrm{m}$ following the PRP $(p=0.005)$. Perceived dyspnoea during the walk test improved from $3.4(1.63)$ to $2.95(1.20)(p=0.004)$ at 7 weeks follow up. $64 \%$ showed a clinically significant improvement in quality of life. The initial total CRDQ score was 63.1 (23.7) and improved to 81.0(27.8) at completion $(p=0.005)$. Those that completed the PRP had a significantly reduced chance of hospital readmission (22\%) compared to the noncompletion group $(48 \%)(p<0.05)$. Conclusions: A 7-week hospital based outpatient pulmonary rehabilitation programme improves exercise tolerance, perceived dyspnoea and quality of life in COPD. Participants who complete the PRP are less likely to be readmitted to hospital. Alternative care models may be needed for non-completers to reduce readmissions.

Key words: pulmonary rehabilitation, quality of life, exercise tolerance Nomination for awards: Respiratory Nurses SIG poster prize
PULMONARY REHABILITATION REDUCES CARER STRAIN AND PSYCHOLOGICAL MORBIDITY

Paul Cafarella and Peter Frith.

Repatriation General Hospital, Daws Rd, Daw Park, South Australia, 5041

Carers, rather than the formal health care system, provide the bulk of personalised long term care. This burden results in physical and emotional health problems (DeLisa and Gans, 1998), and there is a link between a carer's psychological health and the quality of care received by the patient (Draper \& Luscombe, 1998). While the benefits of pulmanary rehabilitation (PR) for patients are well recognised (Mahler, 1998), the effect of PR on the carers of respiratory patients has gone without assessment. Method: A controiled design $(n=100$ ) was used to assess the carers of respiratory patients from the Repatriation General Hospital (S.A.). Fifty carers were assessed before and after their respiratory patients completed PR. Carers in the control group did not have patients who had attended PR. Participants completed the Caregiver Strain Index (CSI), General Health Questionnaire (GHQ) and Katz Adjustment Scales. Results: T-tests for paired samples were used in the preliminary assessment of the data from patients who have currently completed study requirements. The results indicate that carers experience less burden (pre $P R$ mean $=5.22$, sd $=3.44$ vs post $P R$ mean $=4.02, s d=2.73, p<0.001$ ) and lower levels of psychological morbidity (pre $\mathrm{PR}$ mean $=3.72, \mathrm{sd}=5.40$ vs post $\mathrm{PR}$ mean=1.69, $\mathrm{sd}=2.69, \mathrm{p}<0.001$ ) following PR. Conclusions: The results indicate that not only patients, but also carers benefit from PR. The observed carer benefits of reduced strain and psychological morbidity are of relevance given the health problems associated with this role, and are consistent with the findings in nonrespiratory populations (Graham, 1997). Improved carer health status through PR may have positive effects for the care and therefore health of patients with COPD.

Key words: COPD, pulmonary rehabilitation, carer strain, psychological morbidity

OXYGEN THERAPY: CURRENT PRACTICE AND A PRESCRIPTION FOR CHANGE.

Orla Morrissey, Niall Cain, Paul Fogarty \& Frank Thien

Bespiratory Unit, Box Hill Hospital, Victoria 3128

Background: There is evidence for the efficacy of oxygen therapy in certain clinical situations. The aims of this study are to establish the current oxygen prescribing and oxygen saturation monitoring methods in the acute hospital setting and to develop a protocol for the effective and efficient use of oxygen therapy. Methods: An audit sheet and algorithm for oxygen use were designed. Using these sheets, patients on one acute medical (25) and one acute surgical (20) ward had a clinical assessment, which included a pulse oximetry. Analysis of each patient's medical history was performed. Results: $32 \%$ were administered oxygen with no indication. $32 \%$ had a documented $\mathrm{SpO}_{2}$ or $\mathrm{SaO}_{2}$ prior to institution of oxygen. $32 \%$ had oxygen applied by nursing staff with no medical staff order. $20 \%$ had no documentation of who ordered oxygen. Oxygen therapy was ordered on the drug chart in $12 \%$. In terms of $\mathrm{SpO}_{2}$ monitoring, while on oxygen the frequency of assessment was 4-6 hourly in $68 \%$, and in those off oxygen the frequency was $>24$ hourly in $32 \%$. Concluslon: Oxygen is a drug and needs to be prescribed with the same care as other drugs. Based on literature review a protocol for the use and monitoring of oxygen therapy has been devised.

\section{No grant}

Key words: oxygen therapy, prescription, monitoring, audit and protocol Nomination for awards: nil 
EFFECTS OF PULMONARY REHABILITATION ON 12 MINUTE WALK DISTANCE IN LUNG VOLUME REDUCTION SURGERY PATIENTS Jenny Alison ${ }^{1,2}$ and Lissa Spencer ${ }^{1}$

(1) Department of Physiotherapy, Royal Prince Alfred Hospital, Sydney \&

(2) School of Physiotherapy, University of Sydney.

Aim: The aim of the study was to evaluate the effect of an 8 weeks exercise programme prior to lung volume reduction surgery (LVRS) on the distance walked in 12 minutes (12MD) and to compare this with the 12MD following an 8 weeks and 6 months exercise programme post-operatively. Subjects: 25 patients with chronic airflow limitation referred to the pulmonary rehabilitation programme at Royal Prince Alfred Hospital for exercise training prior to LVRS were recruited. Methods: At initial assessment (A) all patients performed spirometry, two 12 minute walking tests (2 days apart) and completed the St George Quality of Life Questionnaire. Each patient was prescribed an individual exercise programme which included walking on the flat, stationary bicycling, arm cranking, upper and lower body weight training, and treadmill walking up an incline. Reassessment at the end of eight weeks training (B) included the same measurements as at $A$. Patients resumed exercise training at an appropriate level 1-3 weeks post LVRS and were reassessed 8 weeks $(C)$ and 6 months later (D). ANOVA was used to compare results at $A, B, C$ and $D$. A $p<0.05$ was considered significant. All data are presented as mean \pm SD. Results: 25 patients with severe airfow limitation (mean $\mathrm{FEV}_{1}=0.62 \pm 0.16 \mathrm{~L}_{\text {; }}$ mean $\mathrm{FEV} / \mathrm{FVC}$ ratio $=33.1$ $10.4 \%$ ) completed the 8 weeks training programme prior to surgery and 8 weeks training post-operatively. There was a mean increase in $12 \mathrm{MD}$ from $A$ to $B$ of $26.5 \%(p<0.001)$ and from $A$ to $C$ of $22.2 \%(p=0.06)$. The subgroup of 11 patients completing 6 months post-operative rehabilitation were reanalysed separately. Improvements in $12 \mathrm{MD} A$ to $B=36.5 \%(p<0.001), A$ to $C=41.4 \%(p<0.05)$ and $A$ to $D=55.3 \%(p<0.001)$. Conclusions: Exercise training in patients with severe airflow limitation significantly improves 12MD. LVRS plus 8 weeks rehabilitation did not further significantly increase exercise capacity in the larger group of 25 patients. Significant improvements in 12MD at 6 months post-operatively in the subgroup of 11 patients suggests that eight weeks post-operatively may be too early to demonstrate peak changes in $12 \mathrm{MD}$ following LVRS.

\section{SIGNIFICANT FUNCTIONAL IMPROVEMENTS AND LONG TERM COMPLIANCE WITH A LOW INTERVENTION HOME BASED PULMONARY REHABILITATION PROGRAM}

R Grimley, A Brazel, B Breust, B Eckert, L Roberts \& D Fielding Department of Respiratory Medicine. Princess Alexandra. Hospital. Qld 4102

Pulmonary rehabilitation programs (PRP) are an established intervention in chronic obstructive lung disease (COLD) with demonstrated efficacy in controlling symptoms and improving functional capacity and quality of life. Home based PRP (HPRP) offer the advantages of improved access, decreased cost and better long-term compliance than hospital based programs. We carried out a prospective study to examine the effectiveness of a very low intervention HPRP.

Methods: 28 patients with stable COLD and breathlessness limiting daily activity underwent a 6 week HPRP. Lung function and functional exercise capacity ( 6 minute walk, endurance walk and maximal stair climb) were measured before and after the program. Exercise was prescribed using a simple algorithm based on performance in the initial functional exercise testing and performed daily at home. Supervision was limited to one initial home visit and a weekly phone call.

Results: The mean FEV1/FVC and FEV1 were $35.6 \%$ and $37.9 \%$ of predicted. 11 patients withdrew because of poor motivation, recurrent exacerbations or loss to follow up. In the 17 subjects completing the program all measures of functional exercise capacity improved significantly: mean six minute walk by $44.4 \mathrm{~m}(p<0.01)$; mean endurance walk by $484.1 \mathrm{~m}$ ( $p<0.001)$; and mean stairs climbed by 10.6 ( $p<0.03$ ). Lung function parameters and results of cardiorespiratory exercise testing did not change significantly. At mean 8.8 months of follow up $13 / 17$ of subjects $(76 \%)$ were still following a regular exercise program.

Conclusions: A simple and low cost HPRP can achieve results comparable to published trials of more intensive PRP as well as good long term compliance. Patients who withdraw may be selected for the more supportive environment of a hospital based PRP.

Key words: pulmonary rehabilitation, chronic obstructive lung disease, home, follow up
THE 24 HR EFFECT OF MANNITOL ON THE CLEARANCE OF MUCUS IN PATIENTS WITH BRONCHIECTASIS.

Evangelia Daviskas', Sandra D Anderson', Stefan Eberl2 ${ }^{2}, \mathrm{H}-\mathrm{Kim} \mathrm{Chan}^{3}{ }^{3}$ Iven $\mathrm{H}$ Young'.

Departments of Respiratory (1) and Pet \& Nuclear Medicine (2) Royal Prince Alfred Hospital, Camperdown, NSW 2050, \& Department of Pharmacy (3), University of Sydney NSW 2006, Australia.

Background \& Aim: We have previously reported an acute increase in clearance of mucus after a single dose of mannitol in patients with bronchiectasis (Daviskas et al. Am J Respir Crit Care Med 1999; 159(6): 1843-1848). In the present study we investigated, in addition to the acute effect of mannitol: 1) the $24 \mathrm{hr}$ retention ; and 2) the clearance rate 24 hours after inhalation of a single dose of mannitol in patients with bronchiectasis. Methods: Clearance of mucus was measured on $\mathbf{3}$ consecutive days in 8 patients with bronchiectasis, age 29 to 70 years. On each day, following inhalation of $99 \mathrm{mTC}$-sulphur colloid aerosol $(6 \mu \mathrm{m})$, lung images were collected over 2 hours and at $24 \mathrm{hr}$. Mannitol $(330 \pm 68 \mathrm{mg})$ was inhaled from an InhalatorTM (Boehringer Ingelheim) only on day 2.

Results: The key findings of the study were that: 1) Mannitol helped patients to clear mucus within $2 \mathrm{hr}$ that would otherwise have taken $24 \mathrm{hr}$ to clear from the whole right lung and all defined regions $(p<0.02)$ and 2) the $24 \mathrm{hr}$ retention of mucus was greatly reduced in all regions when the patients had inhaled the mannitol $(p<0.02)$. The clearance in the peripheral region at 24 $\mathrm{hr}$ was more than double the day mannitol had been inhaled compared to the day without mannitol $(29.1 \pm 4.3$ vs $13.4 \pm 4.6 \%$ ) (p<0.003). However, a single dose of mannitol did not change the clearance rate or lung function beyond $24 \mathrm{hr}(p>0.2)$.

In conclusion, a single dose of mannitol increases clearance of mucus acutely and its effect may extend up to $24 \mathrm{hr}$. The optimum daily dose of mannitol and its long term clinical benefit need to be investigated.

The study has been supported by a NH\&MRC grant

COORDINATION OF BREATHING AND SWALLOWING IN INDIVIDUALS WITH COPD.

Lydia Cvejic 1,2, Richard Harding2, Paul Finlay 1,3, Paul Guy ${ }^{1,3}$ and Nicholas Freezer $r^{1,3}$

1Department of Respiratory Medicine, Monash Medical Centre, 2Department of Physiology, and 3 Centre for Heart and Chest Research, Monash University, Melbourne.

Laryngeal penetration and subsequent aspiration of particulate matter such as food and gastric contents, has the potential to cause lung damage through direct injury and infection. The act of swallowing is intimately coordinated with ventilation in order to minimise this risk. The frequency of aspiration during swallowing in COPD may be increased, however the relationship between ventilation and swallowing in this group has yet to be elucidated. Alm: This pilot study was undertaken to determine if there is (1) an increase in the incidence of laryngeal penetration and aspiration, (2) a decrease in the deglutition-apnoea interval and (3) a change in ventilation before and after swallowing (inspiration-swallow-expiration IES, exp-swallow-exp EES, expswallow-insp EIS and insp-swallow-insp IIS) in patients with COPD. Methods: A group of COPD subjects (median (SD) age 74 years $\pm 4.0, n=5,3 F: 2 \mathrm{M}$ ) with FEV $1 N C<50 \%$ and a group of control subjects ( 71 years $\pm 6.8, n=4,4 F$ ) had videofluoroscopy while swallowing $3 \mathrm{ml}, 5 \mathrm{ml}$ and $10 \mathrm{ml}$ boluses of high density liquid barium. Sternocleidomastoid EMG, respiratory flow at the nose and mouth and pulse oximetry were measured simultaneously. Results: 3 out of the $5 \mathrm{COPD}$ subjects had either laryngeal penetration or aspiration while swallowing at least one of the 3 boluses compared to 0 out of 4 of the control group. There was a consistent respiratory phase difference between COPD and controls (figure1). Inspiration immediately post swallow was only noted in COPD subjects (odds ratio 15:1). No difference was observed in the deglutition-apnoea interval regardless of bolus size or the presence of aspiration $(p=0.2-0.5)$. There was no evidence of arterial oxygen desaturation in any patient. Figure 1.

\begin{tabular}{|l|c|c|c|c|}
\multicolumn{4}{c|}{ Ventilation associated with each swallow. } \\
\cline { 2 - 5 } \multicolumn{1}{c|}{} & IES & EES & EIS & IIS \\
\hline Controls & $83 \%$ & $17 \%$ & $0 \%$ & $0 \%$ \\
\hline COPD & $20 \%$ & $20 \%$ & $60 \%$ & $0 \%$ \\
\hline
\end{tabular}

Conclusions: There appears to be a greater incidence of penetration or aspiration in the COPD group compared to controls in this small group of subjects. This may be due to the increased incidence of inspiration directly following swallowing in the COPD group. There was no difference in the deglutition-apnoea interval. 
TREATMENT OF ACUTE EXACERBATIONS OF CHRONIC OBSTRUCTIVE PULMONARY DISEASE (COPD) IN A WARD-BASED INTERMEDIATE RESPIRATORY CARE UNIT (IRCU)

Cunnington D., Teichtahl H., Humphreys, لـ. Department of Respiratory Medicine, Western Hospital, Victoria, Australia

Background: IRCUs have been used to provide non-irvasive positive pressure ventilation (NIPPV) and basic non-invasive monitoring for acutely ill respiratory patients in non intensive care (ICU) or high dependency (HDU) settings. An IRCU was established in the respiratory ward at our hospital in May 1999. Aims: To evaluate patients admitted to IRCU and determine predictors of outcome in patients with acute exacertations of COPD. Methods: Demographic and physiological data were collected on all admissions to the IRCU. The data were analysed using a multiple logistic regression model to determine predictors of outcome. Results: 60 patients were treated in the IRCU from May to October 1999. 38 patients (68\%) had COPD, $9(15 \%)$ had obstructive sleep apnoea and/or obesity/hypoventilation $(\mathrm{OH})$, and $3(5 \%)$ had bronchiectasis. The remainder had non-hypercapneic respiratory failure due to asthma, pneurnonia or acute pulmonary oedema. NIPPV was used in 30 patients with acute hypercapneic respiratory failure (28 with acute exacerbation of COPD and 2 with $\mathrm{OH})$. Ten of 38 patients with COPD $(26 \%)$ had a poor outcome, defined as death (6 patients) or endotracheal intubation (ET) (4 patients). All of the patients that died had a not for resuscitation order and no patients with a diagnosis other than COPD had a poor outcome. The patients who had a poor outcome were significantly older ( 73 vs 62 years, $\mathrm{p}=0.002$ ) and had a lower initial arterial $\mathrm{pH}(7.19$ vs $7.30, p=0.03$ ) than those COPD patients who did well. In a multiple logistic regression model, age $(p=0.01)$ and initial arterial blood $\mathrm{pH}(\mathrm{p}=0.05)$ were significant predictors of poor outcome in patients with acute exacerbations of COPD.

Conclusion: The IRCU provides NIPPV and specialist respiratory care to patients who may otherwise have required ICU or HDU management. In patients with acute exacerbations of COPD the risk of a poor outcome is related to age and degree of respiratory acidosis at presentation.

Supported by: Western Hospita

Key words; COPD, outcomes, respiratory failure, NIPPV, ward

\section{P99}

THE IMPACT OF HOME OXYGEN THERAPY ON THE DISEASE SPECIFIC QUALITY OF LIFE OF SUBJECTS WITH CHRONIC AIRFLOW LIMITATION.

Alan Crockett'1, Josephine Cranston', John Moss², John Alpers'.

1. Respiratory Unit, Department of Medicine, Flinders Medical Centre, Bedford Park. South Australia, 5042. 2. Department of Public Health. University of Adelaide, South Australia, 5005

Chronic Aiffow Limitation (CAL) is a major contributor to the burden of itlhealth in Australia, and where hypoxia is present, can be treated with home oxygen therapy (HOT). At Flinders Medical Centre, a prospective longitudinal study has been undertaken to examine the impact of HOT on the quality of life of subjects with CAL. Methods: All eligible adult patients, aged less than 80 years, with a primary diagnosis of CAL who met the prescription guidelines of the Thoracic Society of Australia and New Zealand were offered HOT and invited to participate. After baseline assessment subjects were followed-up at 3, 6 and 12 months from commencement of HOT. The disease-specific Chronic Respiratory Disease Questionnaire (CRQ) was applied. A mean change in score from baseline equivalent to 0.5 per question was considered clinically significant. Results: Follow-up was available for 115 CAL patients, (male:female 58:57), mean age 69.3 years, prescribed HOT from January 1991 to July 1999. Based on Guyatt's clinically significant difference for the CRQ, $50 \%$ females experienced significant improvements in CRQ scores at three months, $42 \%$ at 6 months and $43 \%$ at 12 months with $21 \%$ patients experiencing significant deteriorations at 3 months, $22 \%$ at 6 months and $18 \%$ at 12 months. $41 \%$ males experienced clinically significant improvements at 3 months, $33 \%$ at 6 months and $42 \%$ at 12 months with $19 \%$ experiencing clinically significant deteriorations at 3 months and 6 months and $20 \%$ at 12 months.

Conclusion: More CAL patients were able to demonstrate clinically significant improvements in their quality of life after commencing HOT than clinically significant deteriorations.

Key words: Chronic Airflow Limitation, home oxygen therapy, clinically significant difference

\section{A RANDOMISED CONTROLLED TRIAL OF NON-INVASIVE VENTILATION (NIV) IN ACUTE EXACERBATIONS OF CHRONIC AIRFLOW LIMITATION (CAL).}

Con Archis ${ }^{1}$, Mary Dunford1, Michael Lazaris'1, Elizabeth Clark1, Peter Cistulli1,2.

1 Ceentre for Sleep Disorders \& Respiratory Failure. Department of Respiratory Medicine, St George Hospital, \& Unniversity of New South Wales

There is growing evidence that NIV improves outcome in acute exacerbations of CAL. However, the application of this therapy in the ward setting, and its effect on hospital length of stay (LOS) remain to be established. Method: A prospective, randomised controlled trial of standard therapy (ST; ie bronchodilators, steroids, $\mathrm{O}_{2}$, physiotherapy, \pm theophylline) versus ST+NIV in patients presenting to the emergency department with acute hypercapnic $\left(\mathrm{pCO}_{2} \geq 50 \mathrm{mmHg}\right.$ ) exacerbations of $\mathrm{CAL}$. The primary endpoint was the need for intubation and secondary endpoints were lung function parameters, symptom scores (Borg scale) and LOS. Results: 10 patients (mean \pm sem age $75 \pm 6 y r s$ ) with severe acute exacerbations of CAL (FEV1 $0.37 \pm 0.08 \mathrm{~L}, \mathrm{pCO}_{2} 62 \pm 7 \mathrm{mmHg}, \mathrm{pH} 7.33 \pm 0.03$ ) were randomised to either $S T(n=6)$ or $S T+N I V(n=4)$. Half the ST patients failed treatment and were deemed to require intubation, versus none in the NIV group. Symptom scores, FEV1, $\mathrm{FVC}, \mathrm{pCO}_{2}, \mathrm{pO}_{2}$ improved in all subjects by discharge, but the differences between treatment groups were not significant. The average LOS was shorter in the ST+NIV group $(12 \pm 7$ and $6 \pm 2$ days, $p=0.06)$. Conclusion: These preliminary data indicate that ward-based treatment with NIV is feasible in these acutely unwell patients, and further suggest that outcomes (including LOS) may be improved with such therapy. Further evaluation is required to verify these findings.

Key words: CAL, non-invasive ventilation, ward treatment

SEVERITY OF CHRONIC OBSTRUCTIVE PULMONARY DISEASE DETERMINES EFFECTIVENESS OF OUTREACH RESPIRATORY NURSING CARE: A SYSTEMATIC REVIEW

Brian Smitn*, Sarah Appleton*, Bob Adams $\ddagger$, Dick Ruffin*, Ann-Marie Southcott $¥$ *Departments of Medicine and Respiratory Medicine \pm The Queen Elizabeth Hospital, Woodville Rd, Woodville, SA 5011

Respiratory outreach nursing is an alternative care strategy which may reduce the burderi of chronic obstructive pulmonary disease (COPD) in terms of morbidity, mortality and costs to health care systems. Objective: To evaluate the effectiveness of such programmes for patients with COPD in terms of improving lung function, exercise tolerance and health related quality of life (HRQL) of patient and carer, and reducing mortality and hospital service utilisation.

Methods: Randomised controlled trials of relevant studies were identified and assessed according to Cochrane Collaboration guidelines. Results: Four studies were found (total $n=624$ ) with intervention durations of 7-12 months ( 1 and 3 studies respectively). Meta-analysis demonstrated that mortality was not significantly reduced by the intervention, Peto Odds Ratio $0.72 ; 95 \%$ confidence interval $0.43,1.21$. However, post hoc subgroup analysis suggested that mortality was reduced by the intervention in patients with less severe respiratory disease. Significant HRQL improvements were reported in one study in moderate COPD, but not in a study in patients with severe disease. FEV 1 and exercise performance were not improved in the studies where data were available. Hospital admissions, reported in one study in patients with severe disease, were not reduced. Conclusions: Overall meta-analysis showed no benefits from outreach nursing visits for COPD. Post-hoc sub-group analysis indicated that there was improved mortality when more mild COPD subjects were targetted. One study suggests this group also has HRQL gains. Current data suggest there are no reductions in hospital utilisation and health care system costs. Studies of longer duration, enhanced by inclusion of pulmonary rehabilitation may identify subjects most likely to benefit.

Key words: chronic obstructive pulmonary disease, outreach nurse 
BARRIERS TO SUCCESS FOR AN EVIDENCE-BASED "ACCORD" GUIDELINE FOR CHRONIC OBSTRUCTIVE PULMONARY DISEASE (COPD) - THE LITERATURE AND THE EXPERIENCE

Brian J. Smith', Kim Hender', Richard Rutfin'1, Peter Frith², Heather McElroy', Kieran McCaul ${ }^{3}$, Frida Cheok ${ }^{3}$.

(1) Clinical Epidemiology and Health Outcomes Unit. The Queen Elizabeth Hospital. SA 5011 and Dept of Medicine, (2) Respiratory Dept. Flinders Medical Centre, SA 5042, (3) Epidemiology Branch. Dept Human Services. SA 5000 .

An evaluation of barriers and attitudes to best practice guideline use was conducted during a comprehensive in-patient COPD management guideline implementation across three Adelaide metropolitan hospitals. Such guidelines to date have had a limited impact on clinician behaviour and patient outcomes. An evaluation of barriers is important in understanding what drives a clinician to use or not use a guideline. Methods: Focus groups of 30 health practitioners' experience with the ACCORD were analysed using NUDIST software and structured according to the Triandis model 1 , in terms of perceived consequences, emotions, facilitating and social factors. This analysis informed a 44-item questionnaire that was administered one-to-one to all doctors having managed 3 or more COPD patients. The questionnaire included test-retest questions, was piloted $(n=6)$, and modelled using multiple linear regression. Results: Social issues were identified in the focus groups primarily by senior medical staff and specialist respiratory staff, with nursing and allied health reporting more patient-care outcomes. $99(85 \%)$ doctors completed the interview. There was greater guideline use on respiratory units than other units $(p<0.01)$. Senior clinicians used the guideline less $(p=0.02)$ and the $40-50$ year age group were most resistant $(p=0.01)$. Triandis modelling explained $49 \%$ of variance in intention to use the guideline $(p<0.001)$ and $58 \%$ of variance in selfreported use of the guideline $(p<0.001)$. Conclusions: Implementation of guideline use must consider different issues relevant to different health professions, and within the medical profession according to seniority and nature of medical unit. Behaviour prediction modelling explains half of the variance of intention to use and self-reported use of the best practice guideline.

1. Triandis HC Nebraska Symposium on Motivation. 1980; 27:195-259. Supported by the NH\&MRC

Key words: Guidelines, barriers, COPD, attitudes, beliefs.

PREVENTING FRACTURES IN COPD, IS AT LEAST AS COST-EFFECTIVE AS TREATMENT OF POST-MENOPAUSAL. HIP FRACTURES.

S Appleton", B Smith", B Pekarsk ${ }^{\ddagger}$, J Harford *, L Smith", Terry Jones $¥ \ddagger$.

University of Adelaide Department of Medicine," and Pharmacy $\$ \ddagger$ at The Queen Elizabeth Hospital, Woodville. SA. KPMG ${ }^{*}$, Adelaide. Dept of Public Health" ${ }^{* *}$, The University of Adelaide.

Osteoporotic fractures cause significant morbidity, and people with asthma and chronic obstructive pulmonary disease (COPD) are especially at risk. COPD is associated with bone mineral density (BMD) reduction of $10 \%$ with associated increased fracture risk of 2.2. Long-term fracture studies are difficult to conduct, whereas modelling studies can provide information for today. Alendronate has been shown to significantly increase BMD and reduce fracture rate in a number of patient groups. Aims: To model the cost-effectiveness of reducing fractures (\#) by screening the COPD population to identify those with reduced BMD, and treating with alendronate. Methods: Markov model incorporates data for presence/absence of COPD, gender, initial BMD \& treatment response. Decision trees within the model generate annually over 30 years, probabilities of 4 outcomes: no event OR hip fracture OR dying OR institutionalisation. Data were derived from trials, population surveys, health utilisation data and life tables. Results: Costs per hip fracture, death prevented and life years saved were generated for treatment over 10,20 or 30 years (to age 85 ). Table: Describes the number of Australian women with COPD and BMD Z score* $<3$ and also for normal women with $Z=-1 \rightarrow-2$. (Mean BMD in women who have had a hip fracture and are Pharmaceutical Benefits Scheme (PBS) eligible for alendronate.) Conclusion: Screening and targeted preventative treatment for women with COPD is at least as cost-effective as current PBS funded practice. Key words: osteoporosis, hip fracture, COPD.

\begin{tabular}{|l|l|c|c|c|c|}
\hline $\begin{array}{l}\text { Years Tx } \\
\text { (treated) }\end{array}$ & Status & $\begin{array}{l}\text { No. Tx in first year- } \\
\text { No. alive, 85 yr old }\end{array}$ & $\begin{array}{l}\text { Cost/fracture } \\
\text { prevented }\end{array}$ & $\begin{array}{l}\text { Cost/death } \\
\text { prevented }\end{array}$ & $\begin{array}{l}\text { AUS/L } \\
\text { Y saved }\end{array}$ \\
\hline $10(75-85$ yo) & COPD & $114-35$ & $-\$ 1,727$ & $-\$ 5,717$ & $-\$ 1,732$ \\
\hline & hip fracture & $7710-3882$ & $\$ 3,032$ & $\$ 10,019$ & $\$ 1,931$ \\
\hline $20(65-85$ yo) & COPD & $98-15$ & $\$ 920$ & $\$ 4,012$ & $\$ 293$ \\
\hline & hip fracture & $9246-3754$ & $\$ 14,872$ & $\$ 55,324$ & $\$ 7,090$ \\
\hline $30(55-85$ yo) & COPD & $98-13$ & $\$ 7,020$ & $\$ 33,020$ & $\$ 2,088$ \\
\hline & hip fracture & $12303-4633$ & $\$ 38,392$ & $\$ 148,984$ & $\$ 17,262$ \\
\hline
\end{tabular}

* SDs below mean normal, age and gender matched BMD.
SYMPTOMATIC IMPROVEMENTS IN SOME PATIENTS NOT REFLECTED BY IMPROVEMENTS IN FEV : A SYSTEMATIC REVIEW OF THE EFFECTS OF SALMETEROL. IN CHRONIC OBSTRUCTIVE PULMONARY DISEASE.

Sarah Appleton", Brian Smith, Antony Veale $\$$, Anna Bara* Departments of Medicine and Respiratory Medicine ${ }^{\ddagger}$ The Queen Elizabeth Hospital. Woodville Rd. Woodville, SA, 5011. Cochrane Airways Group, Saint George's Hospital Medical School, London

Patients with chronic obstructive pulmonary disease (COPD) often demonstrate little or no reversible airways obstruction. A systematic review was therefore conducted to determine the effectiveness of long acting beta2adrenoceptor agonists (LABAs) on lung function and health related quality of life (HRQL). Methods: Randomised controlled trials (RCTs) comparing treatment with LABAs (salmeterol and formoterol) for at least four weeks, with placebo, in patients with stable non-reversible COPD were identified and assessed according to Cochrane Collaboration guidelines. Results: Four RCTs were found which assessed the efficacy of salmeterol (two 16 week parallel group RCTs and two cross-over RCTs with four week treatment arms). A 16 week study of salmeterol $50 \mu \mathrm{g}$ and $100 \mu \mathrm{g}$ twice daily (b.d.) treatment demonstrated a weighted mean difference for the increase in $\mathrm{FEV}_{1}$ was 0.10 litre [95\% confidence interval $\left.(\mathrm{Cl}): 0.15 ; 0.05\right]$ and 0.12 litre $(95 \% \mathrm{Cl}: 0.17 ; 0.06)$ respectively. $\mathrm{HRQL}$ was significantly improved, using The St George's Respiratory Questionnaire, after $50 \mu \mathrm{g}$ (b.d.), but not after $100 \mu \mathrm{g}$ (b.d). The cross-over RCTs did not show significant increases in FEV 1 or morning and night time PEFR. No improvements were demonstrated in the Medical Outcomes Short Form 36, exercise tolerance (6 minute walk distance) and the incidence of COPD exacerbations. Salmeterol $50 \mu \mathrm{g}$ (b.d.) was associated with reduced Borg Dyspnoea Scores (Peto Odds Ratio $=0.60,95 \% \mathrm{Cl}: 0.40 ; 0.88$ ).

Conclusions: Symptomatic improvements in terms of dyspnoea and HRQL were associated with limited increases in $\mathrm{FEV}_{1}$ in some patients with COPD after 16 weeks salmeterol treatment. The contribution to these improvements by a reversible airways obstruction component in trial subjects is unknown. Key words: chronic obstructive pulmonary disease, HRQL, salmeterol.

SECRETORY LEUKOCYTE PROTEINASE INHIBITOR MAY PROTECT THE LUNG AGAINST THE DEVELOPMENT OF EMPHYSEMA IN INDIVIDUALS WITH ALPHA-1-ANTITRYPSIN DEFICIENCY

Ayad M, Knight $\mathrm{K}^{*}$, Burdon J, Brenton S.

Department of Respiratory Medicine and the 'Bernard O'Brien Institute of Microsurgery, St Vincent's Hospital, Melbourne

Background: We have previously speculated (Respirology 1997;2:91-95) that the variation in expression of alpha-1-antitrypsin (A1AT) deficiency is in part due to the presence or absence of other antiproteinases particularly those of smail molecular size, eg. secretory leukocyte proteinase inhibitor(SLPI) [15kD]. Aim: To present two cases of A1AT deficiency which support this hypothesis. Case studies: Two patients are reported. Each underwent lung function testing (spirometry, DLCO) and bronchoscopy with bronchoalveolar lavage(BAL). A1AP and SLPI were measured in both serum and BAL by standard techniques. Case 1:51 yr old male with severe emphysema (PiZZ) and a past smoking history of $36 \mathrm{pk}$ yrs. Case 2: $49 \mathrm{yr}$ old female with normal lung function but A1AT deficient (PiZZ) and had never smoked.

\begin{tabular}{|c|c|c|c|c|c|c|}
\hline Case & $\begin{array}{c}\text { Smoking } \\
\text { history }\end{array}$ & $\begin{array}{c}\text { Serum } \\
\mathrm{A1} \text { AT } \\
\mathrm{mg} / \mathrm{dL}\end{array}$ & $\begin{array}{c}\text { Serum } \\
\mathrm{SLPI} \\
\mathrm{mg} / \mathrm{L}\end{array}$ & $\begin{array}{c}\mathrm{BAL} \\
\mathrm{A} 1 \mathrm{AT} \\
\mathrm{mg} / \mathrm{mg} \\
\text { Urea }\end{array}$ & $\begin{array}{c}\text { BAL } \\
\text { SLPI } \\
\mathrm{mg} / \mathrm{mg} \\
\text { Urea }\end{array}$ & $\begin{array}{c}\text { Lung } \\
\text { function }\end{array}$ \\
\hline 1 & $36 \mathrm{pk} \mathrm{yr}$ & 0.35 & 0.33 & 0.04 & 1.05 & $\begin{array}{c}\mathrm{AO}, \\
\downarrow \mathrm{DLCO}\end{array}$ \\
\hline 2 & Nil & 0.35 & 0.46 & 0.32 & 2.29 & Normal \\
\hline $\begin{array}{c}\text { Normal } \\
\text { values } \\
\text { (Mean } \\
\pm \mathrm{SD} \text { ) }\end{array}$ & & $\begin{array}{c}1.87 \\
\pm 0.36\end{array}$ & $\begin{array}{c}-114 \\
\pm 0.08\end{array}$ & $\begin{array}{c}0.48 \\
\pm 0.03\end{array}$ & $\begin{array}{c}3.64 \\
\pm 1.94\end{array}$ & \\
\hline
\end{tabular}

\# currentex smokers, data submitted for publication

Conclusion: These resuits are consistent with the concept that SLPI in the presence of A1AT deficiency can protect the lung against the development of emphysema in the absence of cigarette smoking. 
VATS INTRA-CAVITARY ABLATION OF GIANT BULLAE UNDER EPIDURAL FOR HIGH RISK PATIENTS

Gavin M Wright1, C Peter Clarke', Ross D Blair², Graham D Mills ${ }^{3}$

1 Cardiothoracic Care Centre, St Vincent's Hospital, Victoria 3065 2 Thoracic-Vascular Unit and 3 Bespiratory Unit. Waikato Hospital, New Zealand

Unlike lung volume reduction surgery, the benefits of surgery for giant bullae are not in dispute. However some patients have so little reserve that conventional surgery is not a viable option. Two such patients are presented with $F E V_{1}<0.6$ litres and hypercapnia. Both were bed-ridden and severely dyspnoeic on oxygen therapy. A surgical approach was devised by combining several approaches from the literature and adding the use of intra-cavity thoracoscopy. Both patients had surgery under epidural and local anaesthesia with excellent functional results.

A sub-periosteal rib resection was performed, leaving the parietal pleura intact. Concentric purse-string sutures were then placed and lightly snugged to keep the bulla against the parietal pleura and the centre was incised with a scalpel.

$\mathrm{A} 0^{\circ}$ thoracoscope was then advanced to visualize the inside of the bulla, allowing direct identification of septa and bronchial openings. Various techniques could then be used to unify the bullae and control leaks. Talc slurry was sprayed onto the interior surface of the bulla. A 24 French Foley catheter was introduced through the purse-string sutures and its balioon inflated. This was pulled up against the parietal pleura, secured and connected to an underwater seal drain.

Both patients reported immediate symptomatic relief. Improvements in $\mathrm{FEV}_{1}, \mathrm{PaCO}_{2}$ and $\mathrm{X}$-ray appearance were noted. The first patient survived 8 months, requiring oxygen on exertion. The other is fully active at 6 months without oxygen support.

The patients described could easily have been denied a surgical solution for their dyspnoea. This procedure achieves hospital discharge and good quality of life, without imposing risks of thoracotomy, anaesthesia or ventilator dependence.

Key words: Thoracoscopy, emphysema, lung volume reduction surgery.

RISK FACTORS FOR CHRONIC RESPIRATORY DISEASE IN A RURAL ABORIGINAL COMMUNITY IN NORTHERN AUSTRALIA

Graeme Maguire', Stephen McDonald1,2, Norma Benger1 \& Bart Currie1,2 (1) Menzies School of Health Research, Darwin (2) Flinders University. Royal Darwin Hospital Clinical School

Life expectancy for Northern Territory Aboriginal women and men is, respectively, 19.5 and 18.2 years less than non-Aboriginal people. Of this excess mortality $10 \%$ in females and $7 \%$ in males is due to chronic nonspecific lung disease. The cause of this burden of disease is due to factors in addition to tobacco smoking. Methods: Cross-sectional survey of 202 adult residents (18-80 years) of a remote rural Aboriginal community. Spirometry, histamine challenge, respiratory symptoms and signs, and established and postulated risk factors for respiratory disease were assessed. Results: $44 \%$ of participants fulfilled criteria for one or more of chronic bronchitis (CB) (20\%), recent wheeze (RW) $(31 \%)$, or abnormal respiratory auscultation $(18 \%)$. There was no significant difference in gender, age, marijuana use or positive skin prick reaction $(>3 \mathrm{~mm})$ between those with and without respiratory disease. There was a trend for bronchial hyperreactivity (BHR) $\left(\mathrm{PD}_{20} \mathrm{FEV}_{1}<3.8 \mu \mathrm{mo}\right.$ histamine) in those with respiratory disease (12.1 vs $4.4 \% \chi^{2} p=0.08$ ) and current or past tobacco smoking in men only (94.6 vs $81.6 \% \chi^{2} p=0.07$ ). Lower $\mathrm{PD}_{20} \mathrm{FEV}$, for histamine was significantly associated with respiratory disease $(p<0.05)$ and particularly when using a cutoff of $15 \mu \mathrm{mol}$. Low body mass index (BMI) was significantly associated with respiratory disease $(p<0.05)$, particularly those with a BMI in the lower quartile $\left(<20 \mathrm{~kg} / \mathrm{m}^{2}\right)$ $\left(\chi^{2} p<0.01\right)$. CB was associated with increasing age (43.6 (2.8(SEM)) compared to $38.4 \mathrm{yrs}(1.0(\mathrm{SEM})) \mathrm{p}<0.05)$ and also a $\mathrm{BMI}<20 \mathrm{~kg} / \mathrm{m}^{2}$ $\left(\chi^{2} p<0.01\right)$. CB was not associated with tobacco use or $\mathrm{PD}_{20} \mathrm{FEV}_{1}$. BHR $\left(\chi^{2} p<0.01\right)$ and $\mathrm{PD}_{20} \mathrm{FEV}_{1}(p<0.01)$ were however associated with $\mathrm{RW}$ as was current tobacco smoking $\left(\chi^{2} p<0.05\right)$. Conclusion: These findings demonstrate that BHR had a significant association with respiratory disease, particularly recent wheeze. Chronic bronchitis however was not found to be associated with BHR or tobacco smoking in contrast to recent wheeze which was associated with current smoking. The association between BMl and respiratory disease will require further prospective study. Supported by: AMAAPMA Aboriginal Health Initiative, Flinders University Northern Territory Clinical School, Community Health and Anti-Tuberculosis Association, CRC for Aboriginal and Tropical Health. Key words: Indigenous health, chronic non-specific lung disease. Nominations for Awards: nil
THE USE OF ICD CODES FOR ASSESSING OUTCOMES IN COPD Elizabeth A Geelhoed, Philip J Thompson

University Department of Medicine. University of Western Australia and Asthma and Allergy Research Institute

Background: Informational databases provide an expedient means of assessing trends in mortality, disability and costs. Health data are primarily collected according to the International Classification of Diseases (ICD-CM), a coding system based on disease diagnosis. However, Chronic Obstructive Pulmonary Disease (COPD) presents a challenge in diagnostic coding as it may include emphysema, chronic bronchitis and obstructive asthma. Aim:

To assess and analyse the national data set on COPD hospital admissions, classified according to ICD-9. Results: Four years of data from the National Hospital Morbidity Database were examined, using ICD-9-CM codes 490$492 \& 496$ as the primary diagnosis. Most COPD cases were recorded within a residual category labelled 'obstructive disease not otherwise specified' (code 496). The proportion attributed to this category varied from one state to another $(50 \%$ to $80 \%)$. The coding classifications within COPD also demonstrated variation in admission detail. The proportion of admissions for emphysema increased throughout the period ( $8 \%$ to $12 \%$ ) while the proportion for chronic bronchitis decreased (24\% to $19 \%$ ). These trends were also reflected in mortality rates derived from the Australian Bureau of Statistics. The mean length of hospital stay varied across ICD codes, with emphysema (10 days, SEM: 0.2 ) being longer than chronic bronchitis ( 8 days, SEM:0.2) ( $p<.001$ ). Using the number of diagnoses as a proxy for comorbidity, patients with emphysema have significantly more comorbidity than patients with chronic bronchitis ( $p<.001)$. Conclusion: The widespread use of a residual coding category generates both variation in recording because of unclear definition and a lack of information regarding subclassifications of COPD. Retrospective analysis using ICD-9 coding definition for COPD patient groups is likely to provide imprecise outcomes due to the heterogeneity of the most utilised coding group.

Acknowledgements: Astra Pharmaceuticals provides financial support to AARl for Health Economics

Key words: COPD, ICD-9, epidemiology

ACCORD: THE PSYCHOLOGICAL MORBIDITY IN PATIENTS ADMITTED FOR COPD TO SOUTH AUSTRALIAN HOSPITALS.

$P$ Frith, $F$ Cheok, $P$ Walker, N Potts, $R$ Antic, J Alpers, $R$ Ruffin, B Smith, AM Southcott for the ACCORD Project Team

Health Outcomes Unit, SA Department of Human Services, Adelaide, SA 5000

COPD is one of the leading discharge diagnoses for Australian hospitals. The reason for admission is not reflected in the Diagnosis Related Group (DRG) classification; but evidence from overseas and from Flinders Medical Centre (SA) suggest that psychosocial factors are an important contributing factor to admissions. Aim: The Adelaide Collaboration on Chronic Obstructive Respiratory Disease (ACCORD) Project Team developed and implemented evidence-based clinical practice guidelines for use in hospitalised COPD patients, with an aim of identifying more accurately not only physiological but also psychosocial morbidity. Methods: The ACCORD guidelines were implemented in three "active" (A) hospitals over a 6 month period. Patterns of admission and DRGs were tracked before and during the intervention period in both $A$ and "control" (C) hospitals. Patients completed the 28-item General Health Questionnaire at admission, 6 weeks after discharge, and 6 months later; a total score over 5 is considered to indicate significant psychiatric morbidity. Results: Mental health (MH) DRGs were recorded in less than $6 \%$ of patients in the pre-intervention period. At admission $72.5 \%$ of 506 patients had GHQ scores over 5 . Six weeks after discharge $56.2 \%$ of 283 patients had significant psychiatric morbidity, and at 6 months $51.1 \%$ had GHQ $>5$. (The age-matched prevalence of psychiatric morbidity is $12.8 \%$.) The main psychiatric diagnoses defined by the GHQ were Anxiety \& Insomnia (55.7\%) and Severe Depression (30.7\%). Conclusion: There is a high level of psychiatric morbidity in patients admitted for treatment of COPD to SA Teaching Hospitals. Neither the overall psychosocial impact of COPD nor the specific psychiatric diagnoses were coded. This under-recognition of psychosocial morbidity needs urgent attention.

Key words: COPD, hospitalisation, mental health, psychological morbidity 
SYSTEMATIC ASSESSMENT OF CLINICAL PRACTICE GUIDELINES FOR THE MANAGEMENT OF CHRONIC OBSTRUCTIVE PULMONARY DISEASE [COPD]

Kim Hender ${ }^{1}$, Brian Smith ${ }^{1}$, Frida Cheok ${ }^{2}$, Simon Spedding ${ }^{1}$, Peter Frith ${ }^{3}$ \& Alan Crockett ${ }^{3}$

(1)Clinical Epidemiology and Health Outcomes Unit. The Queen Elizabeth Hospital SA 5011. (Murray CJL 1996) (2) Health Qutcomes Unit. Epidemiology Branch. DHS, SA 5000. (3) Respiratory Department. Flinders Medical Centre, SA 5042

COPD is a condition of major mortidity and cost to society. Neither the extent of best practice COPD management guideline uptake in clinical settings, nor the impact on patient health outcomes is known. The quality of development of COPD guidelines is also in doubt. Therefore, our aim was to systematically review COPD guidelines to evaluate the quality of the process of development. Methods: A key-word search was performed using "COPD" and "guidelines". Two reviewers independently evaluated the guidelines using the criteria of Ward ${ }^{1}$ plus medico-legal and ethical considerations on a three-point scale. Results: Six national COPD guidelines (Thoracic Societies of Australia and New Zealand, Britain, USA (X2), Canada and South Africa) and one international COPD guideline (European Thoracic Society) were identified. Inter-reviewer correlations (Kappa $=0.7$ ) showed good agreement. The guidelines were uniformly based upon a consensus approach, mostly with a medical model focus, rather than multi disciplinary, and were lengthy (up to 43 pages) and discursive. Only one had an executive summary suited to everyday practice. The papers did not reflect a systematic evidence-based approach to the collection, appraisal, collation or presentation of information. Most guidelines provided no indication of their level of evidence or methods employed to resolve differences of opinion. There was no evidence of their actual uptake. Ethical and medico-legal implications were not addressed. A single drug company sponsored 6 of the guidelines. Conclusions: COPD is common, and places great morbidity and cost burdens upon society, therefore management principles should follow leading, state-of the-art, best-practice, evidence-based guidelines. However, in spite of guidelines being reported by major national bodies for over a decade now, most fail to meet the majority of Ward's ${ }^{1}$ criteria. Further, there is a lack of evidence of their uptake or any impact upon patient outcomes. There is a need to develop and evaluate independent guidelines, with multi disciplinary involvement, that will be readily adaptable for a range of clinical disciplines.

1. Ward JE, Grieco V. MJA. 1996; 165:574-576.

Key words: COPD, guidelines, development, review, quality of evidence.

A COMPARISON OF SINGLE BREATH CARBON MONOXIDE TRANSFER FACTOR AND HIGH RESOLUTION CT (HRCT) IN THE DIAGNOSIS OF EMPHYSEMA.

CF McDonald, LB Irving, J Usher, PD Rochford, BR Thompson. Department of Respiratory Medicine, Austin and Repatriation Medical Centre, Studley Rd, Heidelberg, Victoria, 3084.

HRCT is the gold standard for the diagnosis of emphysema in patients with airflow obstruction. $A$ reduction in $T_{L} C O$ is also an indicator of the presence of emphysema in this group of patients. A previous study demonstrated a poor correlation between reduction in $T_{2} C O$ and HRCT finding of emphysema in a subgroup of patients with very severe aiflow obstruction (1). Nonetheless, in clinical practice, a reduction in $T_{L} C O$ is commonly interpreted as consistent with a diagnosis of emphysema. We wished to determine the relationship between the reduction of $T_{L} \mathrm{CO}$ in the presence of severe airflow obstruction and the presence of emphysema on HRCT. METHODS: We examined current or ex-smokers with irreversible airflow obstruction, FER $<60 \%$, FEV $1<1.5 \mathrm{~L}, \mathrm{~T}_{\mathrm{L}} \mathrm{CO}<15 \mathrm{~mL} / \mathrm{min} / \mathrm{mmHg}$ who had undergone HRCT. RESULTS: 95 out of 113 patients had reduced $\mathrm{T}_{\mathrm{L}} \mathrm{CO}$ (mean $=37.5 \%$ pred) and $\mathrm{KCO}$ (mean $=50.0 \%$ pred) with $\mathrm{HRCT}$ evidence of emphysema, whereas 18 had reduced $\mathrm{T}_{\mathrm{L}} \mathrm{CO}$ (mean $=53.9 \%$ pred) and $\mathrm{KCO}$ (mean $=64.8 \%$ pred) but no HRCT evidence of emphysema. CONCLUSION: These data suggest a reduction in $T_{1}$ CO does not necessarily equate with parenchymal abnormality in patients with severe smoking related airflow obstruction. The explanation for this reduction in $T_{L} C O$ in patients with severe airflow obstruction but no HRCT evidence of emphysema remains hypothetical. Ventilatory inhomogeneity leading to measurement error may be an explanation for this finding.
THE CLINICAL UTILITY OF EAR LOBE ARTERIALISED CAPILLARY BLOOD IN THE ASSESSMENT OF PATIENTS FOR LONG TERM

\section{OXYGEN}

Eaton TE, Rudkin S, Garrett JE.

Department of Respiratory Services, Green Lane Hospital, New Zealand. Introduction

The prescription of long term oxygen (LTOT) is underpinned by the measurement of arterial $\mathrm{PO}_{2}$, generally obtained by radial artery puncture. Cutaneous oximetry has not proved sufficiently reliable as an alternative. The use of ear lobe arterialised capillary blood has been proposed with several studies suggesting close agreement between arterial values. However, this technique is not widely used and the clinical utility remains uncertain. Methods

Consecutive patients with chronic respiratory disease undergoing assessment for LTOT were invited to participate. Simultaneous radial artery and arterialised ear lobe sampling was performed and procedural difficulties and patient discomfort detailed. Agreement between arterial and arterialised $\mathrm{PO}_{2}$ was compared using the Bland and Altman method.

Results

One hundred patients were studied. Procedural difficulties (insufficient sample or air in sample) were similar for both procedures, however a clotted specimen occurred more frequently in the ear lobe arterialised sample. Seventy six sample pairs were available for comparison. Radial artery and arterialised $\mathrm{PO}_{2}$ were in close agreement with a mean difference $-0.30 \mathrm{kPa}$ $(95 \% \mathrm{Cl}-0.54$ to -0.006$)$. However, based on the arterialised earlobe sample and using the absolute criterion $\left(\mathrm{PO}_{2} \leq 7.3 \mathrm{kPa}\right)$ for the prescription of LTOT, 10/57 (18\%) patients would receive oxygen inappropriately. Conversely $6 / 19(32 \%)$ patients would have been denied treatment. Radial artery puncture gave rise to significantly greater discomfort $(p<0.0001)$ and level of concern $(\mathrm{p}<0.0001)$.

Conclusion

Although patient preference strongly favours arterialised ear lobe sampling, procedural difficulties and insufficient accuracy mean that ear lobe sampling cannot replace radial artery puncture in the assessment of patients for the provision of LTOT.

\section{VALIDATION OF THE SELIM CHRONIC LUNG DISEASE QUESTIONNAIRE WITH OBJECTIVE MEASUREMENT}

\section{Isirgiotis E. Ruffin R. Southcott AM, Smith B. Christopher DJ, Homan S.} Wilson D.Chittleborough C. Department of Respiratory Medicine University of Adelaide, TQEH Campus, South Australia SA 5011

The objective of this study was to evaluate the Selim Chronic Lung Disease Questionnaire (a severity rating based on symptoms) in patients with chronic obstructive pulmonary disease (COPD) and asthma by correlating the Selim score to objective measurements of severity. This open study was conducted in hospital respiratory outpatient clinics. Consecutive patients with COPD and asthma had responses to the Selim Questionnaire recorded: FEV1 and FVC were measured and a patient self rating of severity of illness was documented. The Selim Questionnaire is based on 6 questions related to dyspnoea, cough, wheeze and sputum production, and asks patients to rate these over the previous three month interval. This report is from the initial 55: 32 fernales and 23 males age range 20-79 years. A correlation has been demonstrated between FEV1 and the total Selim score (see diagram).

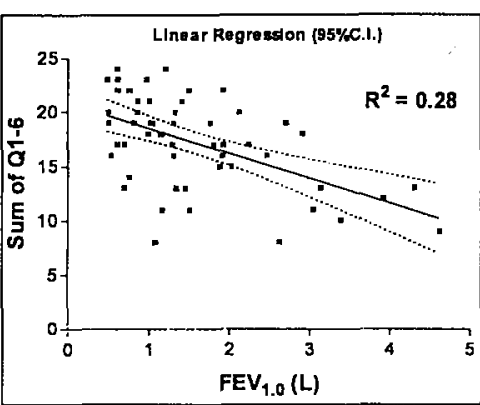

Furthermore, subgroup analysis has suggested that the questions relating to dyspnoea seem to correlate better with FEV1 and FVC than the questions relating to wheezing and cough. It is anticipated that further subgroup analysis will be performed on the basis of diagnostic groups viz. Asthma vs predominantly COPD. This research is funded by
TQEH Research Foundation.

Key words: Quality of life. Selim Questionnaire, Chronic Obstructive

Pulmonary Disease and asthma. 


\section{INTERLEUKIN-1 RECEPTOR ANTAGONIST AND TUMOUR NECROSIS FACTOR $\alpha$ (TNF $\alpha$ ) POLYMORPHISMS IN CYSTIC FIBROSIS}

'Bell SC, 1 Tunnicliffe AM, 18unting JP, ${ }^{1}$ Masel PJ, 2Wolter JM, 2Seeney SL, 2McCormack JG, ${ }^{3}$ Bowler SD, 1Yang IA, 1 Rabnott GC, ${ }^{1}$ Fong KM 1-Adult Cystic Fibrosis Unit. The Prince Charles Hospital: 2 Departments of Medicine, University of Queensland and 3 Thoracic Medicine Mater Adult Hospital, Brisbane

Considerable variation exists in cystic fibrosis (CF) phenotype and severity even amongst individuals with identical CFTR mutations. There is significant individual variation in host inflammatory response, with increased TNF- $\alpha$ and IL-1Ra concentrations in CF airways. Functionally relevant polymorphisms in cytokine genes have recently been characterised. The TNF2 polymorphism at position -308 of the TNF- $\alpha$ promoter is associated with TNF- $\alpha$ higher concentrations in vitro. Variable numbers of tandem repeats in intron 2 of the interleukin-1 receptor antagonist (IL-1Ra), an anti-inflammatory cytokine, lead to alleles which have been associated with inflammatory diseases. We hypothesised that these polymorphisms influence phenotype in CF. Aim: To examine TNF $\alpha$ promoter and IL-1Ra polymorphisms as disease-modifying genes in CF. Methods: We recruited 73 adults with cystic fibrosis and 168 healthy anonymous blood donors. Genotypes were determined using PCR. Results: CF patients (55\% female) had mean (SD) age 25.4 (6.4) years, BMI 21 (3) and $\mathrm{FEV}_{1} 56(22) \%$ predicted. Allele frequencies in CF were TNF2 $19.9 \%$, IL-1Ra*1 $77.4 \%$, IL-1Ra*2 $21.9 \%$ and IL-1Ra*3 $0.7 \%$, which did not differ from controls. BMI was similar between TNF- $\alpha$ genotypes $(1 / 120.9,1 / 2$ $21.2,2 / 2$ 19.8). FEV,$\%$ pred did not differ between TNF- $\alpha$ genotypes (1/1 57\%, $1 / 253 \%, 2 / 267 \%$ ). There was a trend to over-representation of TNF2 in adults with CF who required lung transplantation. IL-1Ra variants were not associated with BMI or lung function. Conclusions: Presently our data do not provide evidence for TNF- $\alpha$ and IL-1Ra polymorphisms as modifiers of BMI, FEV, or $V C$ in CF. Recruitment is continuing to obtain adequate power and further detailed CF phenotyping, including key longitudinal clinical and physiological outcomes, is required to exclude an effect of cytokine gene polymorphisms.

Supported by: The Prince Charles Hospital Foundation Key words: Cystic fibrosis, tumour necrosis factor-alpha, interleukin-1 receptor antagonist, polymorphisms.

\section{P115}

DEFINING AN EXACERBATION OF PULMONARY DISEASE IN CYSTIC FIBROSIS (CF).

Dakin CJ, Henry R, Field P, Morton J.

Respiratory Medicine, Sydney Children's Hospital, Randwick, 2031.

Despite the importance of pulmonary exacerbations (PE) in CF in both clinical and research settings, published evidence or consensus is lacking about the variables used to define an exacerbation. Hospitalisation, a surrogate measure, relies on uniformity among clinicians in diagnosis and treatment of PE. Aims: To evaluate and develop consensus among clinicians about the variables considered helpful in diagnosing a PE requiring treatment. Methods: Two round Delphi consensus development. A comprehensive list of symptoms, signs and investigations used to define PE was compiled from published trials. A written self-administered questionnaire included the list in age appropriate groups to survey opinion about the helpfulness of each item, and the estimated proportion of patients admitted within a month of diagnosis of PE. Sent to all clinicians managing CF patients in Australia. A second round of the survey was sent, with summarised responses from the first, giving the opportunity to modity responses to develop consensus. Results: 1st round: Replies from 59/91 clinicians $(65 \%), 41 / 60(68 \%)$ from those managing children $(C)$ and $18 / 31$ $(58 \%)$ from those managing adults (A). Responses of $\mathrm{C}$ and $\mathrm{A}$ differed for $7 / 32$ variables (MannWhitney, $p<0.05$ ). Clinic grouping did not show greater consensus among responses of $C$ (Kruskal-Wallis $p=0.362$ ). Consensus, $>74 \%$ or $<26 \%$ respondents rating a variable helpful/very helpful, was found in only $50 \%$ of variables listed. Estimated admission rate within a month of diagnosis was $61 \%(30-100 \%)$ for $A$ and $48 \%(5-100 \%)$ for C. 2 nd round: Replies from $55 / 91(60 \%)$, from C $32 / 61(52 \%)$, from A $23 / 31$ (75\%). Consensus increased from $50 \%$ of variables to $80 \%$ in $C_{1}$ and $63 \%$ in $A$. Conclusions: A lack of consensus was found among clinicians managing $C F$ about the variables considered in diagnosing PE and the duration to admission of identified PE's; suggesting inhomogeneity of management. Consensus increased following feedback of summarised responses. The resulting variables will be used to develop a clinical measurement tool.

Key words: Pulmonary exacerbation, cystic fibrosis, delphi. Nominations for Awards: Nil.
EVIDENCE BASED CHEST PHYSIOTHERAPY FOR INFANTS WITH CYSTIC FIBROSIS DIAGNOSED WITH NEWBORN SCREENING Button BM, Heine RG, Catto-Smith AG, Olinsky A, Phelan PD, Ditchfield $M$, Story 1. Depts of Physiotherapy, Gastroenterology, Respiratory Medicine and Radiology, Royal Children's Hospital, University of Melbourne, Alfred Hospital. Newborn screening leads to early diagnosis and introduction of chest physiotherapy (PT) in young mainly asymptomatic infants. Infantile reflux is common in early life. Gastroesophageal reflux (GOR) is more common in CF. Aims: To measure (1) the acute effects of standard postural drainage (SPT) versus modified positions (MPT) on GOR and oximetry in very young infants with $\mathrm{CF}$, while in different states of alertness (SOA) sometimes using non-nutritive sucking (NNS) for comfort; (2) the longer term effects of SPT vs MPT on respiratory status during infancy. Method: 20 infants (mean age 2.1 months) each had 2 sessions of SPT and MPT during $30 \mathrm{hr}$ oesophageal $\mathrm{pH}$ monitoring. The number of reflux episodes (NRE) and oximetry were measured in SPT positions (supine flat, prone, left and right side lying with $30^{\circ}$ head down tilt) and MPT positions (supine $30^{\circ}$ head up, prone, left and right side flat). Different SOA and NNS were recorded. Thereafter infants were randomized to SPT or MPT for 12 months. Respiratory status, medical treatment and radiology were compared. Results: The NRE/hour for SPT, MPT and background $(B G)$ were $2.5 \pm 1.6 ; 1.6 \pm 1.3$; and $1.1 \pm 0.6 ; p<0.05$. The NRE in each position during SPT vs MPT were: supine flat vs supine $30^{\circ}$ head up: 20 episodes vs $12, p=0.067$; prone $30^{\circ}$ head down vs prone flat: 18 vs $9, p=0.03^{*}$; right side lying (RSL) $30^{\circ}$ head down vs RSL flat: 13 vs 9 , $p=0.45$; left side lying (LSL) head down vs $L S L$ flat: 7 vs $11, p=0.59$. The mean SaO2 during SPT vs MPT were: $98.54 \pm 1.60 \%$ vs $98.75 \pm 1.07 \%$; and heart rate $141.97 \pm 11.48$ vs $139 \pm 8.61$ beats per minute. There was no evidence that use of NNS or falling asleep during treatment increased the episodes of GOR. In the longer term study, annual days with respiratory symptoms for SPT versus MPT groups were: cough: $118 \pm 73.56$ vs $83 \pm 119.86, p=0.50$; upper respiratory tract infections: $70 \pm 32.76$ vs $37 \pm 24.91$, $p=0.03^{*}$; wheeze: $41 \pm 32.46$ vs $25 \pm 54.54, p=0.50$; days on antibiotics: $116 \pm 88.56$ vs $67 \pm 58.62, p=0.21$. At mean age $2.5 y r s, 86 \%$ of the intants in the MPT groups had perfect Brasfield scores of 25 versus $43 \%$ in the SPT group. Conclusion: MPT should be considered as the most effective and safe regimen for infants with $\mathrm{CF}$ based on current scientific evidence.

\section{P116}

INFLAMMATION, INFECTION AND LUNG FUNCTION IN CLINICALLY STABLE INFANTS AND YOUNG CHILDREN WITH CYSTIC FIBROSIS (CF). Dakin $\mathrm{CJ}^{11,2}$, Numa $\mathrm{A}^{3}$, Morton $\mathrm{J}^{1,2}$, Wang $\mathrm{H}^{2}$, Henry $\mathrm{R}^{1,2}$. 1. Respiratory Medicine, Sydney Children's Hospital, Randwick, 2031. 2. School of Paediatrics, UNSW, Randwick, 2031. 3. Intensive Care Unit. Sydney Children's Hospital, Randwick, 2031

There is a lack of comprehensive research into interactions between infection, inflammation and lung fünction in infants and children with CF. The aim was to study the effect of lower airway infection on clinical parameters, pulmonary function tests (PFT's), and inflammation in clinically stable patients. Study design was a prospective cohort study of CF patients under 4 years, identified on newborn screening. Methods: A single general anaesthetic, with intubation and muscle relaxation tor PFT's. Passive lung mechanics were measured by single breath occlusion passive deflation (computerised system), for compliance (Crs) and resistance (Rrs). Functional residual capacity (FRC) and total lung capacity (TLC) measured by nitrogen washout. Lavage specimens (BAL) were obtained from right lower, middle and upper lobe bronchi for cytology, cytokine IL8, and quantitative microbiology. Results: 15 children studied, 6 girls, all pancreatic insufficient. Mean age 27.3 months (range 12-42 months). Double $\triangle F 508$ in 12/15. Prior respiratory admissions in $9 / 15$, correlated with culture of Pseudomonas aeruginosa $(\mathrm{Pa})(\mathrm{r}=0.694, \mathrm{p}=0.004)$, and IL.8 level ( $(\mathrm{r} 0.538, p 0.039)$. Lower respiratory pathogens (>10 cfu/ml BAL) were found in 7/15: Staphylococcus aureus (Sa) in 2; $\mathrm{Pa}$ in 4; and $\mathrm{Sa}, \mathrm{Pa}$ and haemophilus in one child. Sa was only detected in children not taking prophylactic flucloxacillin. Culture of any $\mathrm{Pa}$ or $\mathrm{Sa}>105 / \mathrm{ml} \mathrm{BAL}$ correlated significantly with BAL total cell count ( $0.526, p 0.029)$, neutrophil \% (r 0.92 , $p<0.001)$, macrophage $\%(r-0.75, p 0.001)$ and IL.8 $(r 0.72, p 0.001)$. Culture of $\mathrm{Pa}$ correlated with age adjusted $\mathrm{Crs}(r-0.60, \mathrm{p} 0.02), \mathrm{Crs} / \mathrm{FRC}(r-0.69$, $p 0.007)$ and FRC/TLC (0.75, p 0.003). Conclusion: In infants and children with CF the presence of pathogens in the lower airways correlated with levels of inflammation, respiratory system compliance and degree of air trapping.

Key words: Cystic Fibrosis, infant lung function testing, cytokines, intection. Nominations for Awards: Nil 
CYSTIC FIBROSIS CONDUCTANCE REGULATOR GENE MUTATIONS; DO THEY PLAY A ROLE IN THE AETIOLOGY OF ALLERGIC BRONCHOPULMONARY ASPERGILLOSIS?

Tam E Eaton, 1 Patrice W. Miller2, Jeffrey E Garrett', Gary R Cutting 2

1 Department of Respiratory Services, Green Lane Hospital, New Zealand

2 Centre for Medical Genetics and Division of Pulmonary and Critical Care Medicine, Johns Hopkins University School of Medicine, Baltimore

Introduction: Allergic bronchopulmonary aspergillosis (ABPA) shares some important similarities with cystic fibrosis (CF); hence the possibility that genetic factors may play a role in pathogenesis. We aimed to determine the frequency of cystic fibrosis conductance regulator (CFTR) gene mutations in asthmatics with and without $A B P A$.

Methods: Patients were recruited prospectively from asthma clinic. A diagnosis of ABPA required satisfaction of all "essential diagnostic criteria"; asthma, skin prick test (SPT) positivity to Aspergillus fumigatus (Af), elevated serum total $\lg E>1000 \mathrm{ng} / \mathrm{ml}$, elevated serum Af specific lgE, history of pulmonary infiltrates on CXR or central bronchiectasis on CT. Blood samples were obtained from the following patient groups; atopic asthma SPT positive to Af with ABPA ( $n=36$, $(n=27$ previously diagnosed and re-evaluated)), SPT positive to Af without ABPA $(n=26)$, SPT negative to Af $(n=31)$. Genomic DNA was screened for all the commonly occurring CFTR mutations using standard methods.

Results: One of the 26 asthmatics SPT positive to Af without ABPA and 3 of 33 with ABPA were heterozygous for the delta F508 mutation. None of the 31 asthmatics SPT negative to Af carried the delta F508 mutation. Of the less common mutations, only $\mathrm{R} 117 \mathrm{H}$ was identified; in one individual with ABPA and from another asthmatic SPT negative to Af. All patients found to carry a CFTR gene mutation had normal sweat chloride (<40mM).

Conclusion: Extensive genotyping revealed the carrier rate in ABPA cases did not differ significantly from patient groups without ABPA. Indeed it was not different from the anticipated rate in the general population. Hence, the presence of a CFTR mutation does not appear to play a role in the aetiology of ABPA.

This study was supported financially by the Health Research Council of New Zealand and the Green Lane Hospital Research and Education Fund.

GLUCOSE TOLERANCE IN A PAEDIATRIC CYSTIC FIBROSIS CLINIC Jodi Hilton, Dominic Fitzgerald, David Cooper John Hunter Children's Hospital, Newcastle, Australia

Cystic fibrosis related diabetes mellitus (CFRDM), attributed to ongoing pancreatic fibrosis and destruction of beta islet cells, complicates CF in $5-24 \%$ of patients, whilst impaired glucose tolerance (IGT) occurs in up to $75 \%$. The onset of CFRDM is usually insidious and associated with respiratory and nutritional deterioration. There is minimal information on the role of impaired glycaemic control in the period prior to the onset of CFRDM, nor its frequency in the paediatric population. Whilst implied, it remains unclear whether better management of the pre-diabetic stage will translate to slower progression to CFRDM or improved outcomes. Methods: A prospective analysis of glucose tolerance in the CF clinic at John Hunter Children's Hospital, as the first data in screening all school-aged children in NSW. A fasting blood glucose (FBG), 2-hour oral glucose tolerance test (OGTT) and HbA1C were measured. Results: 30 children have completed screening from a clinic of 62 (18 too young, 4 lost to follow-up, 3 CFRDM, 1 pancreatic sufficient, 6 to be completed). Mean [95\% Cl] age was 12.77 years $[11.44,14.1], \mathrm{HbA} 1 \mathrm{C} 5.6 \%[5.46,5.73]$, FBG $4.64 \mathrm{mmol} / \mathrm{L}[4.44,4.84]$ and $2 \mathrm{hr}$ OGTT $7.3 \mathrm{mmol} / \mathrm{L}[6.45,8.14] .11 / 30$ have evidence of IGT on testing with a $1.75 \mathrm{gm} / \mathrm{kg}(\max 75 \mathrm{gm})$ OGTT, with 2 hour BG $9.65 \mathrm{mmol} / \mathrm{L}$ $[8.51,10.82]$. This sepresented an older age group, 14.5 compared to 11.8 years $(p=0.047)$, but there was no significant difference in FBG 4.7 Vs $4.6 \mathrm{mmol} / \mathrm{L}(p=0.64)$, or $\mathrm{HbA1C} 5.7 \mathrm{Vs} 5.54 \%(p=0.31)$. Blood glucose at 2 hours after LucozadeTM load was significantly higher, $9.65 \mathrm{Vs} 5.93 \mathrm{mmol} / \mathrm{L}$ $(p<0.0001)$. Overall there was no influence of enteral feeding or liver disease, but there is a high probability of Type 2 error. Conclusions: In this small initial cohort of children, $37 \%$ have evidence of impaired glucose tolerance on OGTT, whilst only $3 / 11$ had a HbA1C in the IGT range (6.1-7\%). All patients with IGT had FBG $<7.0 \mathrm{mmol} / \mathrm{L}$, including the 1 patient with an OGTT result in the CFRDM range $(14.4 \mathrm{mmol} / \mathrm{L})$. Further evaluation is required of the true incidence of IGT in this population, due to uncertainty regarding influence on morbidity and progression to symptomatic CFRDM.
SEVERE FAT MALABSORPTION IN ADULT CYSTIC FIBROSIS (CF) CAN BE RELIABLY DETECTED WITH ${ }^{13} \mathrm{C}$-TRIOLEIN BREATH TEST HW Greville1, AC Di Matteo2, MA Ritz², RJ Fraser², R Butler ${ }^{3}$, P Cmielewski ${ }^{3}$, G Davidson ${ }^{3}$ Thoracic ${ }^{1}$ and G|2 Medicine Units. Royal Adelaide Hospital, GI Unit ${ }^{3}$, Women's and Children's Hospital. Adelaide, 5000 S.A.

Background: Diagnosis of fat malabsorption in CF patients usually requires faecal collections, invasive procedures or radio-labelled substrates. We aimed to determine whether (i) a non-invasive non-radioactive breath test using ${ }^{13} \mathrm{C}$ triolein reliably detected fat malabsorption in adult CF patients with exocrine pancreatic failure, and (ii) pancreatic replacement therapy (PERT) restored fat absorption to the level of healthy humans. Methods: Studies were performed in 6 adult CF patients $(4 \mathrm{M} ; 29$ yrs (21-36); BMI 22 (21-23)) and 8 healthy volunteers (6M; 24 yrs (20-49); BMI 24 (21-29)). After an overnight fast subjects drank $50 \mathrm{ml}$ of a liquid meal labelled with $200 \mathrm{pl}{ }^{13} \mathrm{C}$ labelled triolein. Breath samples were collected every 30 min for $6 \mathrm{~h}$ by blowing through a straw into collection tubes. In CF patients ${ }^{13} \mathrm{CO}_{2}$ recovery was measured with and without PERT (usual dose for light snack), on different days in a randomised order, and compared to ${ }^{13} \mathrm{CO}_{2}$ recovery from healthy subjects. Intra-individual variability was assessed in 7 healthy subjects by repeating the breath test on a second day (healthy 1and 2). Values are \% cumulative recovery of ${ }^{13} \mathrm{CO}_{2}$ dose over $6 \mathrm{~h}$. (Data are median (IQR); Statistics: Mann Whitney U and Wilcoxon tests; " $p<0.01$ PERT vs no PERT). Results: There was virtually no recovery of ${ }^{13} \mathrm{CO}_{2}$ in CF patients without PERT, but fat absorption was normal (vs healthy controls) after PERT (table). In healthy controls, there was no difference in recovery between days 1 and 2 (table).

\begin{tabular}{|c|c|c|c|}
\hline \multicolumn{2}{|c|}{ CF Patients } & \multicolumn{2}{c|}{ Healthy Volunteers } \\
\hline No PERT & PERT & healthy 1 & healthy 2 \\
\hline $3(0-8)^{*}$ & $\mathbf{3 7}(36-43)$ & $34(22-42)$ & $38(36-44)$ \\
\hline
\end{tabular}

Conclusion: The ${ }^{13 \mathrm{C}}$ triolein breath test is a simple and reproducible method to assess fat absorption which reliably screens for severe fat malabsorption in adult CF patients. Further studies are needed to determine whether it is also useful in less severe pancreatic exocrine failure or monitoring the dose of PERT.

Key words: cystic fibrosis, malabsorption, ${ }^{13} \mathrm{C}$ Triolein breath test

HIGH DOSE INTRAVENOUS TOBRAMYCIN DETERMINED BY AREA UNDER THE CURVE IS WELL TOLERATED IN CYSTIC FIBROSIS

Hopkins P, Bowler S, Rice-McDonald G, Rasiah R

Department of Respiratory Medicine, Mater Adult Hospital, Brisbane Qld 4101

Background: Traditionally, tobramycin is administered at $5-7 \mathrm{mg} / \mathrm{kg} / 24 \mathrm{hrs}$ to treat bronchiectasis in cystic fibrosis (CF) and monitored by trough/peak concentrations.

However, patients with CF exhibit unique pharmacokinetics including increased volume of distribution and clearance, which limit the validity of conventional drug monitoring. Aims: to 1) establish effective doses of tobramycin in CF patients using the Area Under the Curve (AUC) method: 2) document safety by audiometry, urine examination and serum creatinine; and 3) examine determinants of dose requirements.

Methods: Tobramycin was commenced at $10 \mathrm{mg} / \mathrm{kg} / 24 \mathrm{hrs}$ and adjusted according to AUC 86-101 mg/l.hrs (Br.J.Clin.Pharmac. 1995;39: 605-609). Clinical (FEV1,FVC, heart rate, respiratory rate and oxygen saturation), inflammatory (neutrophils, platelet count, CRP, ESR), nutritional (weight, prealbumin), audiometric and renal parameters were measured at baseline and day 10. Results: 15 patients ( 4 male) in 21 episodes of care, mean age 24 median 23 (SD 5.53) with mean FEV1 \% predicted 43.3 median 35.19 (SD 21.47) at day 1 tolerated an average dose of $8.99 \mathrm{mg} / \mathrm{kg}$ median 9.14 (SD 1.76) without any recorded renal nor ototoxicity. Patients achieved mean FEV $1 \%$ predicted of 55.59 median 52.79 (SD 23.44) at day 10. By univariate analysis: body mass index (BMI) $p=0.0003$, serum creatinine $p=0.0004$, FEV $1 \%$ predicted day $1 p=0.01$ and age $p=0.02$ explained $48 \%, 46 \%, 26 \%$ and $20 \%$ of the variability in dose requirements respectively. By multiple adjusted R-squared regression, $60 \%$ of dose variability explained by age, $\mathrm{BMI}$ and serum creatinine. Conclusion: Doses determined by AUC methods are higher, tolerated without increased toxicity and possibly superior to standard regimens. Determinants of dose include age, BMI, severity of lung disease and renal function. 
THEORIES FOR MEDIATING AND NEGOTIATING AN IN-PATIENT STAY FOR FAMILIES AND PATIENTS WITH CYSTIC FIBROSIS

Kathleen Latage', Dr Nicholas Freezer', Associate Professor Jeremy Anderson ${ }^{2}$ \& Professor Alun Jackson ${ }^{3}$

(1) Department of Respiratory Medicine. Monash Medical Centre. Melbourne (2) Centre For Clinical Effectiveness, Monash Medica Centre/Monash University (3) University of Melbourne, Victoria.

Cystic Fibrosis is a life threatening chronic illness requiring many inpatient admissions for treatment. Reviews of the literature and discussions with staff and families reveal a complex array of difficulties associated with the longterm psychosocial management of a chronic illness. Families often bring unresolved issues from past admissions, which may be conflicting in regard to aspects of the treatment or their relationship with hospital staff. These feelings are often expressed in terms of having little power or control in relation to the general management of the illness while in hospital. Staff also speak of a loss of professional role when working with families that are seen to be 'difficult:' A level of stalemate therefore often develops with both families and staff unsure about how they could work more effectively together. Systems theory, feminist and psychodynamic theory can be used to identity and analyse areas of conflict, and structure the process of mediation and negotiation between patients and staff. Systems theory examines the relationships between individuals, the way in which they communicate and the meanings that develop out of those interactions, as well as examining the organisation of the family. Feminist theory examines the structural influences on a family living with a chronic illness and Psychodynamic theory examines the intrapsychic processes of the individual such as the unconscious, the impact of past history on behaviour and object relations theory which examines the bond between child and parent. The use of these theories allows for the development of appropriate interventions to produce an effective outcome for all parties to the process. This in turn may assist families to cope with the demands of a chronic illness as well as potentially reducing unplanned patient presentations.

Key words: Cystic Fibrosis, Mediation, Negotiation

CLINICAL DIAGNOSIS OF CYSTIC FIBROSIS IN A SCREENED

COMMUNITY: 1989-1998

R.J.Massie'1, J Wilson'2, N Freezer ${ }^{3}$, C.F.Robertson ${ }^{1}$

Departments of Respiratory Medicine, Royal Children's Hospital1, The Alfred Hospital2, Monash Medical Centre ${ }^{3}$, Melbourne, Australia.

Introduction: In Victoria $95 \%$ of infants with cystic fibrosis (CF) are diagnosed in the newborn period because of a family history of CF, meconium ileus or newborn screening. Later diagnoses will be of patients born before screening was introduced or represent cases missed by newborn screening. The aim of this study was to examine the presenting features and genotype of patients diagnosed with CF on clinical grounds since the introduction of newborn screening in Victoria.

Methods: A retrospective review of patients attending the $3 \mathrm{CF}$ centres in Victoria (2 paediatric, 1 adult) who were diagnosed with CF between 1989 -1998 following a referral for sweat testing on the clinical suspicion of CF. Results: From 1989-1998, 56 patients were diagnosed with CF on clinical grounds, 47 were born before the introduction of newborn screening and 9 after (missed by screening). The principal clinical presentation of the 56 patients (age range 1 month-40 years) were as follows: failure to thrive(FTT)/steatorrhoea 18 ( 8 missed by newborn screening), productive cough (thaemoptysis) 14 , younger sibling with $C F 6$, rectal prolapse 3 , atypical asthma 3 , nasal polyps 2 , infertility 2 , recurrent pancreatitis 2 , lower respiratory tract infection 2 ( 1 missed by screening), chest pain 1 , hepatosplenomegaly 1 , appendicitis 1 , dehydration 1 . The mean age of the 18 patients with $\mathrm{FTT} /$ steatorrhoea was $2.2( \pm 2 \mathrm{sd})$ years while the mean age of the 14 patients presenting with productive cough was $21( \pm 8 \mathrm{sd})$ years. Nineteen patients were $\triangle F 508 / \Delta F 508$ (mean age at diagnosis $3.5 \pm 3.1$ (sd) years), $24 \Delta \mathrm{F} 508$ /other (mean age at diagnosis 14.3 \pm 11.5 (sd) years), 9 other/other (mean age at diagnosis $12.1 \pm 14.8(\mathrm{sd})$ years), 4 no genotype available.

Conclusions: Younger patients are more likely to present with FTT/steatorrhoea and following the introduction of newborn screening for $\mathrm{CF}$ there may be a lag period of 3-4 years when patients present. Later presentations are more likely to be due to respiratory complications but the range of clinical presentations is broad. Despite the presence of newborn screening for CF a sweat test should be performed if there is clinical suspicion of the diagnosis.

\section{NEWBORN SCREENING FOR CYSTIC FIBROSTS IN VICTORIA: 1989-} 1998

R.J Massie1, M.Olsen², J.Glazner1, C.F.Robertson'1, I.Francis ${ }^{3}$ Department of Respiratory Medicine 1 , Victorian Clinical Genetics Services2, Victorian Newborn Screening Program, Murdoch Institute ${ }^{3}$, Royal Children's Hospital, Melbourne, Victoria, 3052

Background: Newborn screening for cystic fibrosis (CF) was introduced in Victoria in 1989. The primary screen is immunoreactivegtrypsinogen (IRT) measured from heel prick blood taken on day 4 of life. Babies with an elevated ( $>99^{\text {th }}$ centile) IRT had either a second IRT measured at 4-6 weeks (1989-1990) or mutation analysis for $\triangle F 508$ (1991-1998). Babies with an elevated second IRT or with one or two copies of $\triangle F 508$ were referred for a sweat test to confirm the diagnosis of CF. The aim of this study was to review newborn screening for CF in Victoria over the first ten years of the programme (1989-1998) and examine the reasons for missed cases. Method: We retrospectively reviewed the records of the Victorian Newborn Screening Service from January 1989 to December 1998 to ascertain infants identified by newborn screening with CF. We also reviewed the $\mathrm{CF}$ clinic lists of the two tertiary referral paediatric facilities in Victoria to determine if any children born in the years 1989-1998 have been diagnosed outside the newborn period.

Results: From 1989-1998 650070 babies were screened for CF. 221 were diagnosed with $C F$ (incidence $1 / 2942,95 \% \mathrm{Cl} 1 / 3333,1 / 2631$ ), including 30 foetuses detected antenatally. Of the $191 \mathrm{CF}$ babies: 136 were detected by screening, 35 had meconium ileus (MI), 11 were siblings of older children with $\mathrm{CF}$ and 9 babies were missed by screening. The total number of babies detected early (screening, MI, siblings) was 182 which represents $95.3 \%$ of the screened cohort. Of the 9 babies missed by screening, 4 did not have an elevated neonatal IRT, 1 had a repeat IRT at 4-6 weeks which was not elevated (1989-1990), 3 did not have a $\triangle \mathrm{F} 508$ mutation (1991-1998) and 1 had a negative sweat test $(\mathrm{Cl} 36 \mathrm{mmol} / \mathrm{L})$. 7 of the $9(77 \%)$ missed babies were diagnosed within 4 months of birth.

Conclusion: Newborn screening for CF in Victoria has proven effective in detecting most babies with CF in the newborn period. Despite this a sweat test should be requested when clinical features suggest the diagnosis of $\mathrm{CF}$. even if the child has been screened.

\section{BY ELEVATED SURFACE CHLORIDE}

PG Middleton. KA Pollard E Donohoo \& JR Wheatiey

Dept. Respiratory Medicine, Westmead Hospital and University of Sydney, Westmead, 2145, NSW

We have previously demonstrated that the human airway responds to addition of hypertonic saline to the airway surface liquid (ASL) with a decrease in potential difference (PD). This response was rapid, reversible and blocked by amiloride pretreatment, suggesting that the additional saline decreased $\mathrm{Na}^{+}$absorption. To further investigate this response, we have separated the responses to increased $\mathrm{Na}^{+}$, increased $\mathrm{Cl}^{-}$, and increased osmolarity.

Methods: The effect of topical application of various hypertonic solutions on the nasal PD was measured in 6 normal volunteers (Eur Respir J 1994: 7: 2050-2056). Addition of $(500 \mathrm{mM})$ sodium gluconate, $(500 \mathrm{mM}) \mathrm{N}-$ methyl D-glucamine (NMDG)-Cl and (1 M) mannitol to the diluent (Krebs HEPES) were tested on separate days.

Results: Addition of NMDG-Cl decreased PD from a mean (SEM) of $-15.3(1.9)$ to $-4.0(1.0) \mathrm{mV}$, similar to the response to saline $-13.7(1.7)$ to -5.1 (1.3) $\mathrm{mV}$. In contrast, addition of mannitol or sodium gluconate resulted in small increases (more negative) in PD of $1.4(0.8)$ and $2.7(0.9) \mathrm{mV}$ respectively (both $p<0.05$ vs saline).

Discussion: This suggests that the response to hypertonic saline is mediated by the altered $\mathrm{Cl}^{-}$ion concentration, not changes in osmolarity nor $\mathrm{Na}^{+}$ions. As the response to saline is blocked by amiloride pre-treatment, we hypothesise that the airway epithelium can respond to an increase in the ASL $\mathrm{Cl}^{-}$concentration by reflexly decreasing $\mathrm{Na}^{+}$absorption.

Supported by the Australian CF Trust, Westmead Institutes of Health Research Trust. 
AIRWAY GENE TRANSFER IN-VIVO: IMPROVED VIRAL VECTOR TRANSDUCTION AND INCREASED PARTICLE DEPOSITION ONTO AIRWAY EPITHELIUM WITH SIMULTANEOUS LPC TREATMENT.

David Parsons', Peter Hopkins', Tania Martella', Maria Limberis'12 \& James Martin'

(1) Department of Pulmonary Medicine, and (2) Department of Chemical Pathology, Women's and Children's Hospital, Nth Adelaide, S Australia 5006.

A primary limitation to effective airway gene therapy for cystic fibrosis (CF) lung disease is poor gene transfer efficiency. LPC (lysophosphatidylcholine) pretreatment substantially improves adenoviral gene transfer into mouse nasal airway in-vivo. This study assessed the efficacy of coformulations of LPC with vector, and began examination of mechanisms underlying the improvements in gene transfer efficiency. Methods: Reporter gene (AdLacZ) transfer in nasal airways was examined in standard sections after 2 days (XGal processing). A surrogate vector particle - 100nm dia fluorescent beads - was used to estimate vector particle deposition (semi-quantitative scale) onto a defined portion of ciliated nasal septum 30 mins after particle instillation. Results: Vector coformulated with $0.01 \%$ LPC produced enhanced gene transfer (255.8 (82 SEM) transduced cells, $n=5$ ) similar to that observed after pretreatment with LPC (280.3 (34) cells, $n=6 ; p=0.08$, t-test). Various controlgroup instillations (no LPC / irrelevant vector / no vector) produced little gene transter (range 0-50 cells). Compared to coformulation with PBS, LPC cotormulation significantly increased fluorescent particle deposition onto epithelial cell surfaces ( $p=0.03$, Mann-Whitney). Discussion: Preservation of enhanced in-vivo gene transfer when using coformulations of LPC and viral vector indicates LPC does not diminish vector viability, nor does it substantially degrade gene transfer enhancement in-vivo. Increased deposition of LPCcoformulated particles suggests one mechanism is improved vector particle delivery to cells, and we speculate this may be due to solubilisation of airway fluid/mucus, altered receptor access, and direct effects of LPC on other cell functions. Our findings suggest deposition and retention of particles onto respiratory cell surfaces can be modulated by exogenous LPC.

Supported by USA CF Foundation \& WCH Research Foundation

Key words: Gene transfer, adenovirus, nasal airway, particle deposition.

\section{SPUTUM CONTENT OF IRON AND FERRITIN DURING ACUTE} EXACERBATIONS IN PATIENTS WITH CYSTIC FIBROSIS. Reid DW MRCP., Walters EH DM, FRACP., Withers NJ MD., Francis $L$., Wilson JW MD., Kotsimbos TC MD. CF Service, Dept of Respiratory Medicine and Monash University Medical School, Alfred Hospital, Melbourne, Victoria.

Introduction; Colonisation of the cystic fibrosis (CF) airway by Pseudomonas aeruginosa (PA) stimulates the migration into the airway of activated polymorphonuclear neutrophils (PMN) which generate proteases and reactive oxygen species (ROS) which cause cell damage and ultimately lung destruction. Hypothesis; Iron may be part of the patho-immunological processes in CF because of its ability to both facilitate PA replication and catalyse the formation of toxic hydroxyl radicals. Methods; We determined the levels of total iron and ferritin within sputum from 21 CF patients admitted to our institution with an infective exacerbation of their bronchiectasis. We also assessed the sputum concentration of albumin as a marker of vascular leakage. Results: Sputum total iron and ferritin concentrations exceeded those found in normal serum (Table) and significantly correlated with sputum albumin levels $(r=0.7, r=0.6, p<0.05$, respectively). There were significant negative correlations between sputum total iron, ferritin and albumin concentrations and FEV1\%predicted $(r=-0.5, r=-0.5, r=-0.5$, respectively, $p<0.05)$ and FVC\% predicted $\langle r=-0.8, r=-0.8, r=-0.6, p<0.05\rangle$.

\begin{tabular}{|l|c|c|c|}
\hline & $\begin{array}{c}\text { Iron } \\
\mu \mathrm{mol} / \mathrm{L}\end{array}$ & $\begin{array}{c}\text { Ferritin } \\
\mu \mathrm{g} / \mathrm{L}\end{array}$ & $\begin{array}{c}\text { Micro-albumin } \\
\mathrm{mg} / \mathrm{L}\end{array}$ \\
\hline Plasma (NR) & $(13-32)$ & $(15-300)$ & $\mathrm{N} / \mathrm{A}$ \\
\hline CF sputum & 66 & 3801 & 633 \\
& $(13-220)$ & $(525-23,560)$ & $(168-2060)$ \\
\hline
\end{tabular}

NR - normal range, data are expressed as median and (ranges). Discussion; The increased extracellular concentrations of total iron and ferritin in CF sputum demonstrated in this study may facilitate PA colonisation and promote ROS generation thus perpetuating airway damage. Increased sputum albumin concentrations may represent a micro-vascular leak syndrome which contributes to the presence of iron and ferritin within the airway although their very high levels suggest that local production or release of tissue stores may be primarily responsible.

\section{AMINOGLYCOSIDES IN CYSTIC FIBROSIS- A DESCRIPTIVE STUDY OF CURRENT PRACTICE IN AUSTRALIA \\ Judith Phillips, Scott Bell \\ Adult Cystic Fibrosis Unit. The Prince Charles Hospital, Brisbane}

Objective- To determine the diversity of clinical practice with respect to aminoglycosides in Cystic Fibrosis Units within Australia.

Method- In April 1999, a questionnaire was sent to 30 Cystic Fibrosis Units across Australia on the use of Aminoglycosides. Information was collected about drug selection, dosing, monitoring and toxicity with the intravenous and nebulised routes of administration.

Results- Completed surveys were received from 26 of the 30 units (response rate $=86 \%)$ ( $100 \%$ of units with $>40$ patients). There was a wide range of responses received to every question, suggesting that practices vary considerably throughout Australia. Tobramycin was the drug of choice in all but two centres where there was equivalent use of Gentamicin and Tobramycin. The survey demonstrated a trend in recent years to reduce the number of doses per day with $54 \%$ of centres prescribing daily doses for inpatients. Methods of intravenous infusion varied widely, depending on dose, the setting (home or hospital) and venous access device. There was considerable variation in the protocols used for serum level monitoring. Forty percent of centres had computer programs to assist in dose adjustments. In the setting of nebulised antibiotics, Tobramycin was prescribed more often than Colistin; although again there was a wide range of practices evident. Conclusion- The prescribing, dosing, and monitoring of aminoglycosides in Cystic Fibrosis across Australia varies greatly. This may be due to a lack of definitive evidence as to the optimum use of these drugs.

Key words: aminoglycosides, cystic fibrosis, survey, diversity.

AIRWAY RESPONSIVENESS (AR) TO MANNITOL \& $4.5 \% \mathrm{NaCl}$ AEROSOLS IN SUBJECTS WITH CYSTIC FIBROSIS (CF)

Leanne Rodwell', Margaret McElrea', Sandra Anderson², Kim Chan ${ }^{3}$, Scott Bell'.

Depts of (1)Thoracic Med, Prince Charles Hospital, Brisbane 4032: (2)Respiratory Med, Royal Prince Alfred Hospital. Sydney 2050: (3)Pharmacy, Sydney Uni,2006.

Mannitol dry aerosol challenge is used to assess airway responsiveness (AR) in asthmatics. It has not been used to assess AR in CF. Aim: To measure and compare AR to mannitol \& $4.5 \% \mathrm{NaCl}$ in CF subjects. Methods: 12 CF subjects (5F) (18-33yr), inhaled $4.5 \% \mathrm{NaCl}$, generated by DeVilbiss ${ }^{\top \mathrm{M}}$ ultra-neb, for $0.5,1,2,4,8,8 \& 8 \mathrm{~min}$ or part thereof, and on a separate day inhaled mannitol(1) from capsules in $0,5,10,20,40,80,160,160,160 \mathrm{mg}$ doses via a Dinkihaler ${ }^{\mathrm{TM}}$ or Inhalator ${ }^{\mathrm{M}}$. FEV ${ }_{1}$ was measured $1 \mathrm{~min}$ after each dose and salbutamol inhaled after challenge. 8 subjects repeated mannitol(2) challenge. Analysis: Mean $\mathrm{FEV}_{1}$ (\%pred)(SEM) was measured before challenge (Pre), during challenge (Lowest), end of challenge(Final) after salbutamol (postBD). The cumulative dose of saline(ml) \& mannitol(mg) was calculated at the lowest and final FEV ${ }_{1}$. Significance within and between challenges was tested using repeated ANOVA $(p<0.05)$ and t-test with Bonferroni adjustment $(p<0.01)$. Results: The aiway response to $4.5 \% \mathrm{NaCl}$ was different to mannitol( $p<0.028 ; n=12)$. Transient $A R$ occurred during $4.5 \% \mathrm{NaCl}$ challenge, Lowest FEV < Final FEV $_{1}$ : Dose lowest< Dose final) compared to sustained AR during mannitol challenge (Lowest $\mathrm{FEV}_{1}=$ Final $F E V_{1}$ : Dose lowest $=$ Dose final). The mannitol airway response was repeatable $(p=0.736 ; n=8)$.

\begin{tabular}{|c|c|c|c|c|c|c|}
\hline & Pre & Lowest & Final & Post BD & Dose lowest & Dose final \\
\hline$=12$ & $71 \pm 4.3$ & $60 \pm 4.1^{5}$ & $66 \pm 5.1^{*}$ & $77 \pm 4.4^{\wedge}$ & $\overline{11 \pm 3 m !}$ & $27 \pm 3 m \mid \#$ \\
\hline tol(1) $n=12$ & $71 \pm 4.6$ & $62 \pm 3.9^{\mathrm{s}}$ & $63 \pm 4.0$ & $70 \pm 3.9^{n}$ & $495 \pm 50 \mathrm{mg}$ & $575 \pm 30 \mathrm{mg}$ \\
\hline \multicolumn{7}{|c|}{.001 ; 'lowest vs flnal p<0.007; ^flnal vs post BD $p<0.01$; \#lowest vs final p<0.001 } \\
\hline Mannitol(1) $n=8$ & $69 \pm 5.4$ & $58 \pm 4.1^{s}$ & $58 \pm 4.3$ & $68 \pm 4.1$ & $535 \pm 52 \mathrm{mg}$ & $575 \pm 42 \mathrm{mg}$ \\
\hline Mannitol(2) $n=8$ & $67 \pm 5.7$ & $53 \pm 4.6^{5}$ & $54 \pm 4.6$ & $66 \pm 3.7^{\wedge}$ & $495 \pm 47 \mathrm{mg}$ & $555 \pm 43 \mathrm{mg}$ \\
\hline
\end{tabular}

Conclusion: Airway challenges of mannitol dry powder may be better than wet aerosol of $4.5 \% \mathrm{NaCl}$ because it sustains an osmotic gradient longer.

(1)Supported by The Prince Charles Hospital Foundation Key words: Cystic fibrosis, mannitol, airway hyperresponsiveness Nominations for awards: Nil 


\section{ß-ADRENERGIC AGONISTS DO NOT IMPROVE EXERCISE CAPACITY IN} CYSTIC FIBROSIS

D.J. Serisier, A. Coates, S.D. Bowler, \& G. Rice-McDonald Department of Respiratory Medicine, Mater Adult Hospital_South Brisbane. Q 4101

$\beta$-Adrenergic agonists are widely prescribed in patients with cystic fibrosis (CF).The physiologic rationale for their use includes a high incidence of acute bronchodilator reversibility (up to $95 \%$ of patients), improved mucociliary clearance (in-vivo) and increased ciliary beat frequency (invitro). However, there is a paucity of data assessing functional or clinically relevant outcomes, and $\beta$-agonists may reduce alveolar ventilation by increasing dead space. We sought to assess the effect of salbutamol on a functional outcome, exercise capacity, in adults with CF.

METHODS: Randomised, placebo-controlled, double-blind, crossover study of adult CF outpatients with clinically stable disease. Each subject penformed 2 maximal, incremental cycle ergometer exercise tests on separate days following inhalation of either salbutamol or placebo (6 puffs) metered-dose inhaler via a large volume spacer. Each patient, on 2 separate days, underwent histamine challenge and detailed respiratory function testing (with assessment for acute bronchodilator reversibility to salbutamol). RESULTS: 9 unselected patients ( 5 male) aged 17 to 25 were studied. Baseline (mean +/- SD) FEV1 was $71+/-13.5 \%$ of predicted and bodymass index $21.8+1-2.5$. Lung function at the completion of exercise in the salbutamol arm improved significantly from pre-exercise (increase FEV1 $16.1+/-11 \%, p<0.01)$, and this increase was significantly greater than the 5 $+/-3.8 \%$ increase in the placebo arm $(p=0.02)$. There was no difference between the 2 arms for maximal oxygen uptake (salbutamol arm $33.38+1-$ $6.2 \mathrm{ml} / \mathrm{kg} / \mathrm{min}$ vs $32.42+/-2.15, \mathrm{p}=0.6$ ) or maximal workload achieved (salbutamol arm $1185.2+/-280.7 \mathrm{kpm}$ vs $1209+/-272.4, p=0.2$ ). CONCLUSIONS: In this group of adult cystic fibrosis patients, inhaled $\beta$ adrenergic agonists did not result in improved exercise capacity, despite a significant bronchodilation effect. These preliminary results do not provide support for the widespread use of $\beta$-Adrenergic agonists in adults with CF.

Key words: cystic fibrosis, bronchodilator, $\beta$-adrenergic agonists, exercise.
CLINICAL OUTCOME IN PATIENTS WITH CYSTIC FIBROSIS COLONISED WITH BURKHOLDERIA CEPACIA WITH REFERENCE TO SPECIFIC GENOMOVARS.

Soni $\mathrm{R}^{1}$, Marks $\mathrm{G}^{1}$, Robinson $\mathrm{M}^{1}$, Henry $\mathrm{D}^{2}$, Moriarty $\mathrm{C}^{1}$, Parsons $\mathrm{S}^{1}$, Taylor $\mathrm{P}^{3}$, Bye $\mathrm{PI}^{\mathrm{I}}$

(1) Royal Prince Alfred Hospital, Sydney, 2050, Australia. (2) University of British Columbia, Canada. (3) St George Hospital, Sydney, Australia. The presence of colonisation with Burkholderia cepacia (B. cepacia) complex in patients with cystic fibrosis (CF) has been reported to be associated with adverse outcomes. Aims: To determine the actuarial survival, morbidity and post transplantation prognosis of ourpatients with $\mathrm{CF}$ colonised with B. cepacia, with reference to the specific genomovar and strain. To evaluate the effectiveness of the centre's segregation policy. Methods: Retrospective review of spirometric, epidemiological and microbiological data on all patients whose primary CF care was obtained from an adult CF centre and were colonised with B. cepacia. For each B. cepacia colonised subject three age and gender matched case control subjects were randomly allocated. Phenotype and genomovar typing, random amplified polymorphic DNA (RAPD) strain type and B. cepacia epidemic strain marker (BCESM) analyses were performed. The effect of $B$. cepacia-colonisation on transplant-free survival was estimated by Cox's proportional hazards regression using the entire clinic population. Results: Fifteen patients were colonised with B. cepacia of which $6 / 15(40 \%)$ had died from CF related disease by August 1998 compared with 30/173 (17.3\%) of the entire clinic population. Taking the combined end points of transplantation and death $8 / 15$ (53.3\%) of those colonised with B. cepacia compared with $49 / 173(28.3 \%)$ of the clinic population had either been transplanted or died. Cepacia status had a significant adverse effect on survival with a hazard ratio of $2.16(95 \% \mathrm{Cl} 1.0$ to $4.69, p=0.05$ ). When those colonised with $B$. cepacia were sub grouped according to genomovar and strain type: genomovar II was associated with $1 / 2$ (50\%) death, genomovar III RAPD 40, 3/3 (100\%) deaths, genomovar III RAPD $10,1 / 5(20 \%)$ death. The remaining 5 subjects were found to be genomovar III with heterogeneous strains of which $1 / 5(20 \%)$ died and two had lung transplantation and survived. Based on genomovar and strain types there has been no hospital transmission of B. cepacia. Discussion. Colonisation with $B$. cepacia had a significant adverse effect on survival within this study population. Genomovar and strain typing has been used to determine that the segregation policy in place has been effective in preventing cross-colonisation There were trends towards lower pulmonary function and the data suggest prognosis is not uniform amongst specific genomovars and strains. Supported by the NHMRC and CHATA. Nomination for awards: Nil. Key words: Cystic Fibrosis, B. cepacia, survival

AZITHROMYCIN (AZM) REDUCES TNF- $\alpha$ RELEASE FROM LIPOPOLYSACCHARIDE (LPS)-STIMULATED MONONUCLEAR CELLS (PBMC) IN CYSTIC FIBROSIS (CF).

Joanne Wolter1, Sharon Seeney', lan Yang ${ }^{2}$, Simon Bowler ${ }^{3}$, Scott Bell2 \& Joseph McCormack'.

(1) University Department of Medicine and (2) Department of Respiratory Medicine. Mater Adullt Hospital and (3) The Adult Cystic Fibrosis Unit. The Prince Charles Hospital Brişbane.

Use of erythromycin (ERY) for treatment of diffuse pan-bronchiolitis (DPB) follows convincing clinical trials and in vitro evidence of immunomodulatory properties of macrolide antibiotics when given at low dose for long periods. $\mathrm{CF}$ shares many similarities with DPB. AZM is a macrolide antibiotic better tolerated than ERY and is a candidate for long-term oral therapy in CF. We examined the effect of AZM on TNF- $\alpha$ production by LPS-stimulated PBMC collected from 10 healthy individuals and 10 individuals with stable CF. Since TNF- $\alpha$ production is influenced by the presence of polymorphisms for this gene, genotyping was performed using standard PCR techniques. Methods: PBMCs were collected from venous blood and stimulated overnight with LPS $(100 \mathrm{ug} / \mathrm{ml})$ with or without AZM (100ug/ml). TNF- $\alpha$ in cell culture supernatant was measured by ELISA (R\&D Systems). Skewed data were logarithmically transformed and ANOVA applied. Results: There was wide individual variation in TNF- $\alpha$ production. TNF- $\alpha$ levels were higher at baseline and after stimulation in CF-affected cells $(\mathrm{p}=0.02)$. TNF- $\alpha$. significantly increased from baseline after exposure to LPS $(p<0.001)$. The magnitude of change was not different between $C F$ and healthy individuals $(p=0.3)$. Baseline and stimulated secretion of TNF- $\alpha$ was reduced in the presence of AZM in both normal and CF subjects $(p<0.001)$. Change in TNF- $\alpha$ levels after LPS stimulation was 5 -fold greater in one individual homozygous for the variant TNF- $\alpha$, allele than those with the wild type. Conclusions: AZM displays immunomodulatory effects. It inhibits LPS secretion from PBMCs both at baseline and after stimulation with LPS. This effect is not significantly different between individuals with and without CF. Further studies to examine potential immunomodulatory role for AZM in CF are underway. TNF- $\alpha$ polymorphisms appear to influence PBMC response to LPS.

Supported by: Pfizer Pty Ltd

Key words: Azithromycin, cystic fibrosis, TNF- $\alpha$, polymorphism 
NEBULISED HEPARIN IN CYSTIC FIBROSIS

Joanne Wolter ${ }^{1}$, Simon Bowler ${ }^{2}$, Sharon Seeney', Karen Walmsley ${ }^{1}$ \& Joseph McCormack'.

(1) University Department of Medicine and (2) Department of Respiratory Medicine, Mater Adult Hospital, Brisbane.

Heparin is a glycosaminoglycan found naturally in mast cells and is rapidly inactivated by macrophages in the circulation. As an immunomodulatory agent heparin reduces the acute cutaneous reaction to allergens and inhibits bronchospasm in asthma. We studied the effect of this potentially useful therapy on pulmonary inflammation in CF.

Methods: 4 adult CF patients (2 male, mean age 23 (18-25)) chronically colonised by Pseudomonas aeruginosa with stable disease and no history of past severe, or recent haemoptysis inhaled heparin 1000 units $/ \mathrm{kg} /$ day as a single nebulised dose for 10 days. Lung function and sputum samples were assessed at baseline and after the first dose, then again on Day 5 and 10 of therapy. APTT levels were performed on Day 5 and 10. IL-8 in sputum supernatant was measured by ELISA. T-tests compared FEV1, FVC and IL8 levels in sputum.

Results: One patient had mild haemoptysis on Day 7 and ceased treatment. APTT remained in the normal range in all patients. There was no significant change in FEV1 or FVC (\% predicted) between baseline and all subsequent measurements $(p>0.2)$. IL-8 levels in sputum were extremely high (maximum $200 \mathrm{ng} / \mathrm{ml}$ ). There was wide intra-individual variation in IL-8 levels detected in sputum samples. No significant difference in IL-8 levels was demonstrated during or after heparin therapy $(p>0.18)$.

Conclusion: In this pilot group, short-term (10 days) nebulised heparin therapy was well tolerated but was not associated with any detectable improvement in lung function or reduction in LL-8 in sputum.

Key words: cystic fibrosis, heparin, IL-8

\section{LUNG CANCER AND ASBESTOS EXPOSURE IN VICTORIA - PRELIMINARY RESULTS}

O'Halloran J, Burdon J

Department of Respiratory Medicine, St Vincent's Hospital,Melbourne

Background: Lung cancer is a common malignancy in Australia. Whilst cigarette smoking is regarded as being the causative factor in most patients it is also true that most are not subjected to a detailed comprehensive occupational history.Aim: The aim of this study is to determine the incidence of asbestos in patients presenting with lung cancer. Method: All patients presenting with histologically proven lung cancer to the Depts of Respiratory Medicine, Cardio-Thoracic Surgery and General Medicine at St Vincent's Hospital were identified at presentation. Detailed occupational and smoking histories were taken. Demographic data (age, sex, ethnic background) was recorded. Matched control patients without lung cancer but suffering from ather respiratory conditions were similarly interviewed. The data collected were stored and analysed using the MicroSoft Access Database. Results: The results of the first 24 matched pairs enrolled in this study are presented. There were 4 female and 20 male pairs with a mean age 59.8yrs (range 52-69yrs) and 69.5yrs (range 53-83yrs) respectively. Mean smoking history was respectively 56.8 and $57.9 \mathrm{pk}$ yrs in the controls and lung cancer patients. Seven lung cancer patients and 10 controls had had asbestos exposure (no significant difference). Conclusions: The results of the analysis of the first 24 patient pairs enrolled in this study indicate that there is no excess exposure to asbestos in lung cancer patients compared with controls.

\section{ENDOBRONCHIAL TECHNIQUES IN MALIGNANT MAIN AIRWAY OBSTRUCTION}

\author{
Caldecott M., Robinson P. \\ Department of Thoracic Medicine, Royal Adelaide Hospital
}

Introduction A variety of bronchoscopic techniques is available at our institution for the palliation of iroperable locally advanced airway malignancy or to improve survival in lung carcinoma in situ. This study is a review of the use of Nd:YAG laser ablation and airway stents in such patients. It aims to establish their efficacy at our institution compared to other previously reported series. Method: We retrospectively reviewed the records of 36 patients treated for progressive ainway malignancy (26 primary lung) with Nd:YAG laser ablation $(n=34)$ and/or stent insertion $(n=16)$. 33 patients had advanced inoperable tumours and 3 had lung carcinoma in situ. Results: Successful palliation was achieved following $93 \%$ of $\mathrm{Nd}$ :YAG laser ablations and $81 \%$ of stent insertions. At 6 months symptomatic benefit was maintained in $45 \%$ post stent and $29 \%$ post laser. For all procedures, patients with primary bronchogenic carcinoma achieved a shorter duration of benefit. In this group radiotherapy post procedure was effective with $47 \%$ maintaining local control at 4 months compared with $25 \%$ without adjuvant radiotherapy. 24 patients died during follow up, 13 from progressive airway obstruction, 8 from generalised disease and 2 from immediate procedure related complication. In patients with lung cancer, the presence of stage 4 disease pre procedure adversely affected survival. Kaplan-Meier survival curves will be presented for censoring. Conclusion: Nd:YAG laser therapy and airway stents are both effective, safe procedures for the palliation of symptomatic malignant airway obstruction as previously reported in larger patient series. Adjuvant radiotherapy was demonstrated to be beneficial. Selection of appropriate lung cancer patients should favour those without distant metastases. There is probably also a survival benefit although a prospective, randomised clinical study would be required to further evaluate this.

Key words: Nd:YAG laser, airway stent, malignancy 


\section{CHARACTERISTICS OF PATIENTS WITH LUNG CANCER UNDER THE} AGE OF 45

M Cornere, J Kolbe, W Fergusson, T Christmas

Respiratory Services, Green Lane Hospital, Auckland, New Zealand

Introduction: Lung cancer represents $12 \%$ of all cancers registered in New Zealand and $20 \%$ of all cancer deaths. The incidence of lung cancer, like other countries parallels the rate of cigarette smoking and is increasing. Smoking rates among teenagers are increasing raising concerns that this may result in increased rates of lung cancer in young people. The aims of this study was to determine the characteristics of young $(<45 y \mathrm{r})$ patients with lung cancer.

Methods: A case control study was undertaken. Cases were patients aged $<45$ diagnosed with primary lung cancer at Green Lane Hospital between 1993 and 1998. Controls were chosen by matching for the date of the diagnostic procedure. Clinical and pathological data were obtained retrospectively from clinical notes.

Results: 48 patients (<45yrs) were diagnosed as having lung cancer. These were matched with 123 control subjects $(>45 y$ rs). The mean age was 38 compared to 67 in the control group $(p<0.01$ ). There were $67 \%$ females versus $32 \%$ in the control group. Adenocarcinoma was the predominant type $(48 \%$ v $27 \%)(p=0.001)$ whereas squamous cell carcinoma was less common $(17 \%$ v $35 \%)(P=0.001)$. The majority of cases were current or exsmokers $(80 \%)$ compared with $95 \%$ of control. Mean survival overall was 11.1 versus 8.5 months $(p=0.1)$. There was no difference in tumour stage, family history or ethnicity between the two groups.

Conclusion: These data suggest young ( $<45 \mathrm{yrs}$ ) patients with lung cancer are predominately female and adenocarcinoma is the most common histological diagnosis. Smoking is the likely aetiology but other factors (genetic) may contribute. The data may also suggest that young women may be more susceptible to the carcinogenic effects of cigarette smoke.

PRE-OPERATIVE PREDICTORS OF COMPLICATIONS POST LUNG CANCER (CA) RESECTION: LESSONS FROM LUNG VOLUME REDUCTION SURGERY (LVRS).

E. Gabbay, C. Watson, M. Edwards. Dept of Respiratory Medicine and Cardiothoracic Surgery, Royal Perth Hospital, Western Australia.

The best pre-operative predictor of postoperative complications following lung resection remains unclear. Results from LVRS suggest that patients with severe emphysema can safely undergo thoracic resection of 'target' areas of lung with poor perfusion suggesting that even patients with severe emphysema and lung CA may be operable. We have elected to perform CA resection on patients with severe emphysema (FEV1 $<35 \%$ predicted), who would previously have been deemed inoperable, where it could be combined with unilateral LVRS. Between February and October 1999, surgery was performed on 5 patients with lung $\mathrm{CA}_{\text {, }}$ an FEV $1<35 \%$ predicted and appropriate 'target' areas of poor perfusion. Pre-operative and perioperative data are shown.

\begin{tabular}{|c|c|c|c|c|c|}
\hline & FEV 1* $^{*}$ & DLCO $^{*}$ & VO2 max & operation & Inpatient days \\
\hline 1 & 27 & 31 & 54 & (R) upper lobectomy & 24 \\
\hline 2 & 25 & 40 & 51 & Wedge resection & 21 \\
\hline 3 & 29 & 35 & 49 & $(\mathrm{R})$ upper lobectomy & 17 \\
\hline 4 & 32 & 30 & 60 & $\begin{array}{c}\text { (R) upper + middle } \\
\text { lobectomy }\end{array}$ & 36 \\
\hline 5 & 23 & 37 & 47 & Wedge resection & 27 \\
\hline
\end{tabular}

* \% predicted.

Median (range) improvement in FEV1 was $24 \%(-3,46 ; p=0.23)$. There have been no deaths and none of the patients has required long-term oxygen. Two patients had prolonged ( $>21$ days) air leak and two required postoperative intravenous antibiotics. There were no episodes of reintubaton and all patients were discharged from the intensive care unit within 48 hours. We conclude that the applicability of previously established risk factors for lung resection may need to be reassessed.

\section{ABERRANT GENE PROMOTER METHYLATION IN NON-SMALL CELL.} LUNG CANCER (NSCLC)

Fong KM1 1 , Zoechbauer-Mueller $\$^{2}$, Virmani $A^{2}$, Geradts $\mathrm{J}^{2}$, Gazdar $A F^{2}$, Zimmerman PV 1 , Minna JD²

1Division of Thuracic Medicine, The Prince Charles Hospital; 2UT Southwestern Medical Center, Dallas, Texas. USA

Aberrant methylation of $\mathrm{CpG}$ islands in the promoter region of genes is a tumour acquired mechanism for inactivating the function of tumour suppressor genes. Aim: To determine the frequency of geme promoter methylation of the following genes: p16, O6-methylguanine-DNAmethyltransferase (MGMT), death-associated protein kinase (DAPK), Ecadherin (ECAD), and glutathione S-transferase P1 (GSTP1). Methods: DNA was obtained from 107 resected NSCLC and corresponding normal lung tissue. After bisulphite modification, unique PCR primers allowed the detection of methylated DNA sequences from unmethylated sequences. Results: Aberrant promoter methylation was identified in $25 \%$ for $016,18 \%$ for ECAD, $19 \%$ for DAPK, $6 \%$ for GSTP1, and $20 \%$ for MGMT, whereas it was not seen (or only at very weak levels in the corresponding normal tissues). At least one of these five genes were aberrantly methylated in $62 \%$ of resected NSCLC. As expected, aberrant methylation of p16 corresponded with p16 down-regulation, as detected immunohistochemically. Correlations for various aberrant methylation changes include other genetic changes, disease stage and survival. Conclusions: This study confirms that a large proportion of NSCLCs are characterised by aberrant promoter methylation in the five genes tested. As other genes are known to be aberrantly methylated in other human cancers, it is likely that more genes will be identified to be aberrantly methylated in lung cancer. If so, not only will methylation studies contribute to knowledge of the pathogenesis of lung cancer, but may also provide an early detection strategy as others have found that aberrant methylation can also be detected in the peripheral blood of lung cancer patients.

Supported by:

Key words: lung cancer, molecular, promoter methylation, tumour suppressor genes

\section{A COMBINED DIAGNOSTIC AND PLEURODESIS ROLE OF MEDICAL THORACOSCOPY USING FROZEN SECTION}

Hopkins P1, Fielding D1, Solomos $\mathrm{J}^{2}$, Steele $\mathrm{R}^{3}$

Departments of Respiratory Medicine (1), Anaesthetics(2), Anatomical

Pathology (3) Princess Alexandra Hospital, Brisbane, Queensland 4102

Background and Aims: Medical thoracoscopy has high diagnostic efficacy in pleural effusions and the potential to perform pleurodesis in the same procedure, thereby reducing the number of patient interventions. Because pleurodesis should be proceeded by tissue diagnosis, we investigated the role of frozen section at the time of thoracoscopy to determine if this could allow more combined procedures.

Methods: Prospective evaluation of 11 patients with undiagnosed symptomatic pieural effusions. All underwent medical thoracoscopy under local anaesthesia and conscious sedation in the bronchoscopy suite. Biopsies for frozen section and conventional histology were submitted at thoracoscopy. In addition, closed pleural biopsies (CPB) were taken through the entrance port before proceeding to compare diagnostic sensitivity. Results: 11 patients had final diagnoses of 1) 6 benign and 2) 5 malignant ( 4 mesothelioma and 1 pleural synovial sarcoma). Frozen section concurred with the final histopathological diagnosis in 10/11 subjects, with 1 mesothelioma associated with extensive adipose tissue not detected. The diagnostic sensitivity for malignancy at thoracoscopy was $100 \%$; the 6 benign cases have shown no evidence of disease progression or recurrence (mean follow up 4 months). In 7/11 subjects CPB results were consistent with thoracoscopic biopsies, with a sensitivity of only $40 \%$ for malignancy. One benign case along with ail malignancies were pleurodesed with good radiological outcome. One patient with mesothelioma has a chronic pleurocutaneous fistulae at the intercostal catheter site undergoing slow resolution. The patient with mesothelioma and negative frozen section was pleurodesed at a second thoracoscopy three weeks later. Conclusions: Medical thoracoscopy is the preferred intervention in pleural effusions in which the aetiology remains undetermined after simple thoracentesis. Frozen section analysis during the procedure facilitates the possibility of doing combined procedures. 
AUDIT OF CLOSED PLEURAL BIOPSY LEADING TO AN ALGORITHM FOR THE MANAGEMENT OF PLEURAL EFFUSIONS USING MEDICAL THORACOSCOPY

Hopkins $P_{\text {, Fielding } D}$

Department of Respiratory Medicine, Princess Alexandra Hospital, Brisbane

Background: An efficient diagnostic approach to exudative pleural effusions after an initial negative thoracentesis remains controversial. We conducted a retrospective review of patient records at the Princess Alexandra Hospital from 1994-99: 104 subjects who had undergone closed pleural biopsy (CPB) with thoracentesis for pleural effusion. Aim: 1) audit CPB with respect to diagnostic sensitivity and procedural aspects 2) consider the role of CPB in the diagnosis and management of pleural effusion. Results: Of 104 patients, 51 had final diagnoses of malignancy, 44 benign and 9 no final diagnosis. Adequate pleura was sampled in $82 / 104$ subjects $(79 \%)$. Only $30 / 51$ (59\%) malignancies were detected from the combined procedure of cytology pleural fluid and CPB with 19 requiring thoracoscopic or open pleural biopsy (OPB) and 2 bronchoscopy. Sensitivity of $\mathrm{CPB} /$ cytology for mesothelioma was $37 \%$ and the negative predictive value of a nonspecific biopsy $59 \%$. In $39 \%$ of procedures, no sedation or analgesia was administered and $32 \%$ of biopsy specimens were not sent for microscopy. The sensitivity of taking $1 \mathrm{CPB}$ was $0 \%, 2-4$ $51 \%$ and taking multiple biopsies $57 \%$. Increasing volume of pleural fluid sent for cytological examination correlated with improved diagnostic sensitivity for malignancy. The complication rate was $16 \%$. Conclusions: Procedural - The sensitivity of a single pleural biopsy is low and not appreciably improved beyond 2-4 percutaneous samples. There is a greater need for analgesia and sedation use during CPB along with a need to send specimens for microscopy and culture. Pleural fluid sent for cytology should be of at least $100 \mathrm{ml}$ volume for improved diagnostic sensitivity. Diagnostic Role - Our reported sensitivity of CPB is similar to previous published studies. Nonspecific biopsy results are common and pose a significant limitation to this technique given the low negative predictive value for malignancy, particularly mesothelioma. At our institution this has led to an algorithm including medical thoracoscopy in a combined diagnostic and pleurodesis role.

\section{TRANSFORMING GROWTH FACTOR (TGF) $\beta_{2}$ PRODUCES MORE} EFFECTIVE AND FASTER PLEURODESIS THAN TALC

YC Gary Lee, KB Lane, LR Teixeira, MAC Vaz, AO Carmo, CJ Devin, FS Vargas, Richard W Light \& Philip J Thompson University of Western Australia, W.A.; University of Sao Paulo, Brazil and St. Thomas HospitalVanderbilt University, USA.

TGF $\beta$ is a unique cytokine with potent pro-fibrotic and anti-inflammatory properties that make it an attractive agent for pleurodesis. We hypothesise that TGF $\beta$ can produce pleural fibrosis effectively, quickly and without causing excessive pleural inflammation. Aims: To compare the 1) degree and 2) speed of pleurodesis and 3) histologic changes after intrapleural instillation of TGF $\beta_{2}$ and talc. Methods: 18 rabbits were given either TGF $\beta_{2}$ $(1.7 \mu \mathrm{g})$ or talc slurry $(400 \mathrm{mg} / \mathrm{kg})$ via a chest tube. 3 rabbits from each group were sacrificed at Days 1,4 and 7 . Pleurodesis was graded macroscopically from 1 (none) to 8 ( $>50 \%$ hemithorax). Histologically, pleural inflammation and fibrosis were graded from 0 to 4. Pleural thickening and area of collagen deposition were measured. Results: TGF $\beta_{2}$ induced excellent pleurodesis within 7 days. It produced more collagen deposition and pleural fibrosis. $\mathrm{TGF}_{2}$ also stimulated the production of large effusions of low inflammatory indices.

\begin{tabular}{|c|c|c|c|c|c|c|c|c|c|}
\hline \multirow{6}{*}{ 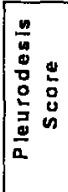 } & $\square$ Talc & - & & \multicolumn{3}{|c|}{ Effusion } & \multicolumn{2}{|c|}{ TGF $\beta_{2}$} & Talc \\
\hline & & & & Vol. 2 & $\mathrm{ir}$ & & $18 \pm$ & & $2 \pm 0$ \\
\hline & & & & \multicolumn{3}{|c|}{ WBC $\left(100 / \mathrm{mm}^{3}\right)^{\star}$} & $12+2$ & & $243 \pm 64$ \\
\hline & & & & \multicolumn{3}{|c|}{$\mathrm{LDH}(100 \mathrm{IU} / \mathrm{L}))^{*}$} & \multicolumn{2}{|c|}{$30 \pm 6$} & $118 \pm 25$ \\
\hline & & $\because$ & & Prote & in & & $29 \pm$ & & $29 \pm 2$ \\
\hline & $\bar{\delta} \overline{\bar{y}}$ & & $\sqrt{47}$ & \multicolumn{3}{|l|}{${ }^{*}$} & \multicolumn{3}{|c|}{$\mathrm{Me}$} \\
\hline \multirow{2}{*}{\multicolumn{3}{|c|}{ ean values shown. }} & \multicolumn{2}{|c|}{ Day 1} & \multicolumn{3}{|c|}{ Day 4} & \multicolumn{2}{|c|}{ Day 7} \\
\hline & & & Talc & $\mathrm{TGFB}_{2}$ & Tale & & & \multicolumn{2}{|c|}{ Talc TGF $\beta_{2}$} \\
\hline \multirow{2}{*}{\multicolumn{3}{|c|}{ Teural Fibrosis $(0-4)$}} & & 2.0 & & & & 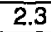 & 3.0 \\
\hline & & & 1.0 & 1.3 & ? & & & 1. & $\overline{3.3}$ \\
\hline \multirow{2}{*}{\multicolumn{3}{|c|}{$\begin{array}{l}\text { eural thickness } \quad(\mu \mathrm{m}) \\
\text { tal Collagen }\left(1000 \mu \mathrm{m}^{2}\right)\end{array}$}} & 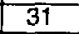 & 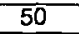 & 132 & & & 78 & $34 \overline{0}$ \\
\hline & & & 12 & 51 & 27 & 21 & 17 & 76 & 576 \\
\hline
\end{tabular}

Conclusions: $\mathrm{TGF}_{2}$ produces pleurodesis more effectively and faster than talc. $\mathrm{TGF}_{2}$ induced more collagen deposition and fibrosis without causing more inflammation.
OUTCOME AFTER SURGICAL RESECTION OF NON-SMALL CELL LUNG CANCER (NSCLC) IN THE ELDERLY. B H Lam*, C Kennedy, L Truong, B C McCaughan, P N Hendel, M J Peters. Concord Repatriation General Hospital. Royal Prince Alfred. Hospital and Strathfield Private Hospital, NSW.

Surgical resection offers the only realistic prospect of long-term survival in NSCLC. In NSW, half of all NSCLC is diagnosed in patients over 70. The presence of significant co-morbidity and previously reported high operative risks may dissuade clinicians from considering surgery in older patients. Methods We reviewed data collected prospectively on tumour staging, surgery performed and outcome in patients from CRGH and RPAH(1985-98) and SPH (1989-98). All hospitals have in common an 'aggressive' approach to surgery. Mortality included death from any cause and is not agecorrected. Results Data were available on 455 patients. The table summarises results presented by operation and stage. In 18 cases resection was impossible. 30 day mortality was $4.2 \%(n=19)$. Common causes of death were cardiac $(n=4)$ or respiratory complications (7) and CVA (5). Mortality after lobectomy fell from $7.1 \%$ for $1985-93$ to $2.9 \%$ for

\begin{tabular}{|l|c|c|c|c|c|}
\hline & Cases & $\begin{array}{c}30 \text { day } \\
\text { Mortality }\end{array}$ & $\begin{array}{c}1 \text { year } \\
\text { Survival }\end{array}$ & $\begin{array}{c}2 \text { year } \\
\text { Survival }\end{array}$ & $\begin{array}{c}5 \text { year } \\
\text { Survival }\end{array}$ \\
\hline Pneumonectomy- $R$ & 19 & $10.5 \%$ & $32 \%$ & $26 \%$ & $8 \%$ \\
\hline Pneumonectomy- $L$ & 45 & $4.4 \%$ & $63 \%$ & $48 \%$ & $22 \%$ \\
\hline Bilobectomy & 34 & 0 & $86 \%$ & $64 \%$ & $27 \%$ \\
\hline Lobectomy & 258 & $4.7 \%$ & $72 \%$ & $59 \%$ & $33 \%$ \\
\hline Wedge Resection & 70 & $1.6 \%$ & $74 \%$ & $67 \%$ & $35 \%$ \\
\hline \hline Stage 1A & 100 & $6.3 \%$ & $83 \%$ & $80 \%$ & $54 \%$ \\
\hline Stage 1B & 182 & $4.0 \%$ & $72 \%$ & $64 \%$ & $35 \%$ \\
\hline Stage 2 & 79 & $2.6 \%$ & $72 \%$ & $50 \%$ & $21 \%$ \\
\hline Stage 3 & 67 & $3.1 \%$ & $56 \%$ & $34 \%$ & $6 \%$ \\
\hline
\end{tabular}

1994-1998 and was higher with right, $7.5 \%$, than left-sided lobectomy, $1.6 \%$ $(p=0.02)$. Conclusions Surgery is the treatment of choice for many elderly patients with NSCLC. Operative risks have fallen and are now lower than frequently reported. Operative risks are highest and outcomes are poor after right pneumonectomy. In this older group, there is no difference in survival between lobectomy and wedge resection.

Key words: Bronchogenic carcinoma, surgery, elderly, survival

GROWTH FACTOR INDUCED EXPRESSION OF METALLO-PROTEINASE 2 IN CELLS CULTURED FROM PATIENTS WITH PLEURAL MESOTHELIOMA

H Parsons, M Tamm, P. Johnson, R Himbeck, B Robinson, J Black. M Roth Department of Pharmacology, University of Sydney and Department of Respiratory Medicine, Perth, Australia

Mesothelioma develops in people exposed to asbestos and is characterised by a spread throughout the pleural space with tissue infiltration. In contrast to other lung carcinomas showing distant organ metastases early in the clinical course mesothelioma develops as a locally very aggressive tumour. Tumour proliferation is influenced by the composition of the extracellular matrix (ECM). ECM is regulated by de novo production, degradation by matrix metalloproteinases (MMP) and the action of the respective inhibitors. Furthermore different growth factors contribute to malignant cell proliferation. To investigate the role of MMPs in tumour progression in mesothelioma we analysed the supernatant of human mesothelioma cell lines under cell culture conditions for their ability to synthesise MMPs in the presence and absence of growth factors. Zymography and western blot were performed as previously described (AJRCCM 1997;156:1987). Three out of 5 primary human mesothelioma cell lines spontaneously synthesised MMP-2 propeptide but no other gelatinolytic MMP, especially no MMP-9. The MMP-2 pro-peptide could not be activated by the mesothelioma cells themselves. Following stimulation with transforming growth factor B MMP-2 expression increased in all mesothelioma. In contrast, stimulation with platelet derived growth factor did not influence MMP-2 expression.

Summary and conclusion: MMP-2 is expressed by cultured human mesothelioma cells, especially if stimulated with transforming growth factor B. This might explain the pattern of local tumour progression in mesothelioma. In contrast, platelet derived growth factor had no effect on MMP 2 production. Our findings might allow to develop new therapeutic approaches to control tumour spread in patients with mesothelioma. This study is supported by the Dust Diseases Board of New South Wales and the Australian Lung Foundation 


\section{CT-GUIDED FINE NEEDLE ASPIRATION BIOPSY OF INTRATHORACIC} MASSES

\author{
Biha, RL, Rafter, LE, Slaughter, RE, Oliver, WA \\ The Prince Charles Hospital, Queensland, Australia
}

Background: CT has been established as the most accurate method for guiding fine needle aspiration (FNA) biopsies of intrathoracic masses, thereby allowing for cytological delineation of malignant from nonmalignant lesions. In January 1997, a database was established at a tertiary referral hospital specialising in cardiothoracic disorders to assess procedural outcomes and to quantify individual risk. Method: During a 2.5-year period (01.01.1997-30.06.1999), 315 FNA biopsies were performed on 312 patients (202 males, 110 females). Biopsies were performed with $3-5 \mathrm{mls}$ of 1-\% lignocaine injected into skin. A Greene 19g-biopsy needle was used under CT guidance and samples aspirated via a finer gauge needle passed through the outer needle. Data were recorded at the time of patient referral and procedure and were followed up. Results: The mean age of patients was $65+12.4$ yrs (range $19.1-89.7 y$ rs). Forty-nine (16\%) patients were aspirin/NSAIDs; $11(3.5 \%)$ on anticoagulants. Diagnostic material was obtained in $72.7 \%$ of samples. Lung cancer represented 201 samples ( $96 \%$ NSCLC, 4\% SCLC). Other malignancy was diagnosed in $22(7 \%)$ cases. Cellular atypia was evident in $17(5 \%)$ cases. Benign lesions were diagnosed in $6(2 \%)$ cases. Non-specific features characterised $71(22.5 \%)$ samples. Eleven aspirates proved false-ve and 4 false+ve for malignancy. The only complications recorded were pneumothorax in $40 \%$ of cases $(17 \%$ requiring ICC) and haemoptysis in 3 cases. The mean \% predicted values for FEV1, VC and KCO did not correlate significantly with risk of pneumothorax. Conclusions: CT-guided FNA biopsy continues to be a reliable method of diagnosing intrathoracic lesions, thereby reducing the need for diagnostic thoracotomy. Diagnostic accuracy is high in lung cancer, though negative results do not preclude malignancy. Benign cytology may be of use in a clinical context but is rarely useful in establishing a diagnosis. Pneumothorax, though a frequent complication, is significant in a relatively small number of patients.

Acknowledgments: Sr D. Gillan

Key words: lung cancer diagnosis; fine-needle aspiration biopsy; pneumothorax

\section{CARCINOMA OF THE BRONCHUS AND HIV INFECTION}

Selwyn Fung, Peter Wark*, Paul S. Thomas. Faculty of Medicine, UNSW \& Dept of Respiratory Medicine, Prince of Wales Hospital, Randwick, 2031. "Airway Research Centre, John Hunter Hospital, Newcastle, 2310.

It is well established that lymphomas and cutaneous malignancies, such as Kaposi's sarcoma and squamous cell carcinoma, are a common sequelae of human immunodeficiency virus (HIV) infection. Carcinoma of the bronchus is less clearly associated with HIV. A review was therefore undertaken of HIV positive patients who presented with a diagnosis of carcinoma of the bronchus, and compared with a group of control patients who were not in a risk group for HIV. Methods: Case notes of all patients coded as both HIV +ve and lung cancer between the period from $1 / 1 / 96$ to $31 / 12 / 98$ were reviewed at $\mathbf{2}$ hospitals which are local centres for HIV care. For each case, 10 controls were matched for sex and compared for age, smoking, histopathology, and survival time. Results: Five patients who were HIV +ve were identified with biopsy proved primary lung cancer. All were male smokers. Median CD4 count was 180, range 120-500.106/L. Histopathology: large cell 2; squamous cell 2; \& 1 adenocarcinoma. Survival time was brief, median 3-6 months, but all received palliative radiotherapy; 2 also required steroids and one required a pulmonary artery stent. Lung cancer was diagnosed at a median age of $49 \mathrm{yrs}$ (range 37-53 yrs), and 2-9 yrs after diagnosis of HIV. Compared to the control group there was a significantly earlier median age of presentation; controls: 71yrs (42-81yrs, $p<0.0001 \mathrm{chi}$ squared). Conclusion: Bronchial carcinoma occurs at an earlier age in those with HIV infection, and has a poor prognosis.

Key words: HIV, lung cancer
A CLINICO-PATHOLOGICAL REVIEW OF PRIMARY PULMONARY NONHODGKINS LYMPHOMA

Andrew Scott ${ }^{1}$, Richard Slaughter ${ }^{2}$, Peter Wood ${ }^{3}$, Belinda Clarke ${ }^{4}$

1The Department of Thoracic Medicine, 2Director of Radiology, ${ }^{3 D i r e c t o r ~ o f ~}$ Haematology, ${ }^{4}$ Director of Anatomic Pathology, The Prince Charles Hospital, Brisbane.

Objectives: To evaluate the clinical, radiological and pathological profile of disease in patients with primary pulmonary non-Hodgkins lymphoma in nonimmunocompromised patients. Methods: A retrospective review of patients with histologically proven disease, between 1973 and 1999. 22/patients had confirmed lymphoma without extrathoracic involvement. Records were available for 18 patients. Pathology and radiology were reviewed by independent specialists. Two patients with primary pulmonary Hodgkins disease were not included.

Results : Low grade (LG) lymphomas were found in 6 male and 3 female patients (median age 70 years) who presented with cough $(67 \%)$, dyspnoea $(33 \%)$, weight loss $(22 \%)$, B symptoms $(11 \%)$ and haemoptysis $(11 \%)$. Mean ESR at presentation 40.3. Imaging demonstrated localised nodules / opacities $(89 \%)$, adenopathy (22\%), pleural effusion (11\%) and diffuse infiltrates (11\%) without cavitation. Bronchoscopy revealed mucosal involvement in $22 \%$. Monoclonal gammopathy (IgM and $\mathrm{IgH}$ ) involved the marrow in $22 \%$ of patients. Treatment included surgery alone (22\%), chemotherapy ( $44 \%$ ), and supportive (22\%). Survival (1 year) was $75 \%$, and at 3 years $71 \%$. High grade $(\mathrm{HG})$ lymphomas occurred in 7 male and 2 female patients (median age 44 years). There was a higher incidence of weight loss (89\%) and 8 symptoms (56\%). Mean ESR at presentation 67.6 . Hypercalcaemia was common (67\%). The major radiological pattern was nodules (67\%) with cavitation in $33 \%$. Additional patterns included pleural effusions $(33 \%)$, diffuse infiltrates (33\%) and adenopathy (11\%). Mucosal abnormalities on bronchoscopy included extrinsic compression (33\%), and a polypoid mass $(11 \%)$. No marrow abnormalities were detected. Treatment included surgery $(11 \%)$, adjuvant chemotherapy $(22 \%)$, or chemotherapy alone $(44 \%)$. One year survival was $25 \%$.

Conclusions: Patients with HG lymphomas were younger and more symptomatic on presentation. Both groups had predominant nodular patterns on imaging. Poorer outcome was associated with HG tumours, weight loss and B symptoms, elevated ESR and hypercalcaemia, but not serum LDH. LG tumours were more responsive to combined chemotherapy (alone or adjuvant) with better survival outcomes.

Key words: Non-Hodgkins lymphoma, primary lung malignancy, chemotherapy

ADENOVIRAL MEDIATED GENE TRANSFER OF ACHAETE-SCUTE HOMOLOG-1 INDUCES APOPTOSIS IN NON-SMALL-CELL. LUNG CANCER (NSCLC)

DN Watkins, V Sriuranpong, MW Borges, BD Nelkin, SB Baylin, DW Ball. Johns Hopkins Oncology Center, Baltimore, MD, USA.

Introduction. The achaete-scute complex of basic helix-loop-helix transcription factors determines neural cell fate in Drosophila development. A mammalian achaete-scute homolog (MASH1) is essential for neuroendocrine (NE) cell differentiation in the developing lung. A human ortholog (hASH1) is highly expressed in small cell lung cancer (SCLC) and has been implicated in the pathogenesis of the malignant NE phenotype. Though these findings suggest a role for hASH1 in commitment to NE cell fate in airway development and repair, the effects of hASH1 on cell growth and differentiation in non-NE airway epithelial cells are unknown. Aim. Using a replication deficient adenovirus strategy, we studied the effects of hASH-1 overexpression in two classic hASH1 positive SCLC lines (NCl-H209, DMS53), two variant SCLC hASH1 negative lines ( $\mathrm{NCl}-\mathrm{H} 82, \mathrm{NCl}-\mathrm{H} 417$ ) and two hASH-1 negative NSCLC lines (A549, U1752). Methods. Cells were infected with an adenovirus containing hASH1, or control viruses expressing either a hASH1 mutant deficient in the basic DNA binding domain, or betagalactosidase. Viral dose was normalized for the expression of a green fluorescent protein reporter by FACS analysis. Cells were examined for changes in morphology, growth and nuclear condensation by Hoechst staining. Results. Both control viruses had no effect in all 6 cell lines. Infection with the hASH1 adenovirus induced growth arrest and apoptosis in both NSCLC lines, but induced no change in growth or morphology in the classic and variant NSCLC lines. Conclusion. These data suggest that hASH1 specifically induces apoptosis in NSCLC. This effect is likely to be transcriptionally mediated given the lack of apoptosis induced by the hASH1 mutant lacking DNA binding capability. This unexpected finding suggests an intolerance to the induction of a NE differentiation program in NSCLC, and implicates specific anti-apoptotic mechanisms in SCLC necessary for the expression of the NE phenotype. 


\section{A CASE OF GOOD RESPONSE TO ROXITHROMYCIN FOR DIFFUSE PANBRONCHIOLITIS}

Con Archis', Leon Vonthethoff ${ }^{2}$, Elizabeth Clark'

Departments of 1 Respiratory Medicine and 2Anatomical Pathology, St George Hospital. Sydney, NSW.

Diffuse panbronchiolitis is a chronic progressive pan-inflammatory condition affecting the respiratory bronchioles. It is most commonly seen in Japanese people and rarely reported outside the Far East. The condition has a poor prognosis due to the inefficiency of corticosteroids although erythromycin has been shown to be effective. Case: We report a case in a Chinese-born man who presented with symptomatic airway obstruction and nodular infiltrate on radiological investigation. Diagnosis was subsequently made on open lung biopsy and he was treated with roxithromycin $300 \mathrm{mg}$ daily. Clinically and radiologically he has had a good response after three months therapy. His lung function has improved with FEV1 of 1.2 (pre-diagnosis) to 2.5L, and FVC of 2.7 to $3.7 \mathrm{~L}$, with less air-trapping but unchanged indices of diffusion. Conclusion: Roxithromycin has been used successfully in this patient. It should be considered as an alternative therapy to erythromycin Once daily administration and better tolerance may improve patient compliance.

Key words: diffuse panbronchiolitis, roxithromycin

\section{ASTHMA IN NEW ZEALAND SAWMILL WORKERS}

Jeroen Douwes, Dave McLean, Tania Slater, Neil Pearce

Wellington Asthma Research Group, Wellington School of Medicine, New Zealand

Exposure to both hard- and softwood dust has been shown to be associated with occupational asthma, chronic airflow obstruction, and respiratory symptoms.

We conducted a cross sectional questionnaire study among 772 New Zealand sawmill workers (processing pinus radiata) focussing on respiratory symptoms.

Preliminary results showed that asthma prevalence (current asthma medication, or shortness of breath or asthma attack(s) in past 12 months) in sawmill workers with moderate to high exposure to wood dust (based on job title) was significantly higher $(19 \% ; n=623)$ compared to non- or low wooddust-exposed workers $(12 \% ; n=147)(O R=1.8 ; p<0.05)$. Non-exposed workers included office workers $(n=52)$, saw doctors $(n=27)$ and various other job titles with an expected low exposure to wood dust $(n=68)$. Twenty two percent of the moderate to high exposure workers reported wheezing, shortness of breath or chest tightness in relation to certain work tasks compared to only $8 \%$ among the low or non-exposed workers $(\mathrm{OR}=3$; $\mathrm{p}<0.001)$. Also cough and cough with phlegm were reported more frequently $(\mathrm{OR}=1.8$ and $1.7 ; p<0.05)$ by the moderate to high exposure workers $(34 \%$ and $28 \%$ versus $22 \%$ and $18 \%$, respectively). Significant differences in smoking habits (but not in age and duration of employment) were found between both exposure groups. After adjusting for smoking, odds ratios for asthma and asthma symptoms in relation to work remained the same, whereas odds ratios for cough and cough with phlegm were only marginally decreased ( $O R=1.7$ for both symptoms; $p<0.05$ ).

In conclusion, preliminary results indicated that exposure to pine wood dust is associated with asthma and other airway symptoms.

Supported by the Health Research Council of New Zealand. Key words: Occupation, epidemiology, respiratory symptoms, sawmill workers
THE INVESTIGATION OF CRYPTOGENIC FIBROSING ALVEOLITIS (CFA): RESULTS OF THE CFA PRACTICE SURVEY

S Chapman (1), G Ryan (2), R Scicchitano (1), M Wilsher (3), FR Lake (4) (1) Department of Thoracic Medicine. Royal Adelaide Hospital, Adelaide. 5000 (2) Department of Respiratory Medicine. Sir Charles Gairdner Hospital, Nedlands, 6009 (3) Department of Reespiratory Meedicine, Green Lane Hospital, Auckland. New Zealand (4) Department of Medicine, Royal Perth Hospital, Perth, 6001

CFA is the most common cause of interstitial lung disease. Previous surveys of pulmonary physicians have shown little consistency in the diagnostic procedures used for, or the methods for monitoring progress in, CFA. The development of further diagnostic tools for CFA such as HRCT chest and DTPA scanning are likely to have had an impact on the evaluation of CFA. This prompted us to evaluate the investigation of CFA in Australia and New Zealand. Methods: A questionnaire was designed to evaluate the patterns of investigation and treatment of CFA using an example case of fibrotic CFA. This was distributed to all identified respiratory physicians in Australia and New Zealand. Results: 168 responses to the questionnaire have been received. When investigating a case of probable CFA, $>90 \%$ of respondents would routinely perform CXR, HRCT chest, spirometry and DLCO, while $<10 \%$ would routinely perform open lung biopsy (OLB), gallium scan, DTPA scan or measured VO2(max). The investigations considered most important in making a diagnosis of CFA were HRCT chest, DLCO and OLB. The investigations considered most useful for monitoring progress of CFA were DLCO, spirometry and static lung volumes. In the example case of fibrotic CFA, $55 \%$ of respondents would recommend a biopsy, with $61 \%$ of these recommending a thorascopic or open biopsy. Conclusions: HRCT chest is now considered to be a routine part of the investigation of CFA, while the use of routine open biopsy has declined. Although $<10 \%$ of physicians indicated they routinely perform OLB, $55 \%$ indicated they would perform it in a hypothetical case of fibrotic CFA, suggesting a discrepancy between perceived and actual practice.

Key words: Interstitial lung disease, investigation, lung biopsy, questionnaire.

\section{EXHALED NITRIC OXIDE IN INFANTS AND DOMESTIC FORMALDEHYDE EXPOSURE.}

Peter Franklin', Stephen Stick²

(1) Department of Paediatrics, University of Western Australia

(2) Department of Respiratory Medicine, Princess Margaret Hospital for Children, WA, 6008.

Formaldehyde is an ubiquitous indoor air pollutant. We have previously reported an association between residential formaldehyde concentrations and exhaled nitric oxide levels (eNO) in healthy children. We are currently investigating if a similar association can be demonstrated in infants. Methods: Formaldehyde was monitored in homes using passive sampling devices that were placed in the living room and child's bedroom for at least 3 days. The infants $(n=20)$ made one visit to the Respiratory Medicine Department at Princess Margaret Hospital where they underwent a lung function test using the raised volume-rapid thoracic compression technique. Exhaled air was collected in gas sampling bags during tidal breathing and eNO was measured from the bags using a nitric oxide analyser (Seivers NOA280). Results: There was no effect of formaldehyde levels on lung function, however a significant correlation was found between eNO levels and indoor formaldehyde concentrations $(r=0.48, p<0.05)$. Conclusions: These are preliminary data and the number of subjects is small, however this supports our previous findings that exposure to formaldehyde at leveis typically encountered in the home may induce an inflammatory response in the airways of children.

Supported by the NH\&MRC

Key words: Formaldehyde, exhaled nitric oxide, infants Nominations for awards: nil 
MANAGEMENT OF CRYPTOGENIC FIBROSING ALVEOLITIS (CFA) IN AUSTRALIA AND NEW ZEALAND: TREATMENT PATTERNS

FR Lake (1), S Chapman (2), G Ryan (3), R Scicchitano (2), M Wilsher (4) (1) University Department of Medicine. Royal Perth Hospital, Perth, 6001 (2) Department of Thoracic Medicine, Royal Adelaide Hospital, Adelaide, 5000 (3) Department of Respiratory Medicine. Sir Charles Gairdner Hospital. Nedlands 6002. (4) Department of Respiratory Medicine, Green Lane Hospital, Auckland

Evidence supporting the use of drugs in the treatment of CFA is limited with no placebo controlled trials being performed. More recently, HRCT has helped stratify patients into those with a poorer prognosis and poorer response to treatment (honeycombing on HRCT vs ground glass changes). However as the prognosis is poor, many physicians feet a need to treat patients with CFA. Methods: Using a postal questionnaire, respiratory physicians in Australia and New Zealand were asked about treatment of CFA using an example case of fibrotic CFA. The patient was a 65yo man with moderate symptoms for 6 months, exercise limitation to $100 \mathrm{~m}$, moderately impaired lung function ( $50 \%$ predicted) and honeycombing on HRCT. Results: 168 responses to the questionnaire have been received. In regard to the sample case, $74 \%$ of respondents would recommend treatment following the results of initial investigations, while a further $23 \%$ would start treatment if there were a decline in symptoms or lung function. Only $3 \%$ would never commence treatment, the reasons being fibrosis on HRCT and lack of evidence for effectiveness of treatment. Of the 163 who would consider treatment, the majority $(76 \%)$ would use Prednisolone/Prednisone (Pred) alone as initial therapy, 15\%

Pred/Cyclophosphamide and 6\% Pred/Azathioprine. In addition, $3 \%$ would use methylprednisolone in the initial phase. If the patient deteriorated, the most common alternatives ( $>25 \%$ of respondents) considered were azathioprine, cyclophosphamide, cyclosporin and colchicine. Conclusions: A pro-active approach to treatment of a hypothetical case of fibrotic CFA is demonstrated in Australia and New Zealand. This may reflect the lack of good evidence supporting or refuting the role of therapy in CFA and a poor prognosis in this disease.

Key words: Cryptogenic fibrosing alveolitis, drug therapy, questionnaire.

\section{AMIODARONE LUNG TOXICITY: A CASE SERIES}

J Malone, J Burdon, R Brownlee*

Departments of Respiratory Medicine and Radiology*, St Vincent's Hospital, Melbourne

Background: Amiodarone toxicity is an unusual cause of diffuse parenchymal lung disease.Case studies: A series of four cases is presented, for which causation was either proven on biopsy or strongly suggested by clinical features. The clinical,radiological and histologic features are described in detail, and for three patients, serial respiratory function testing is reported. In all cases, a mixed alveolar and interstitial infiltrate was present. One case had severe hepatitis, and was also notable for the presence of marked peripheral blood eosinophilia. This patient developed pulmonary fibrosis and still required domiciliary oxygen five months after cessation of amiodarone. The remaining patients had complete resolution of their pulmonary infiltrates, however two had reduced carbon monoxide transfer documented at the end of the follow up period. Three patients were treated with corticosteroids. In the remaining case,corticosteroid therapy was contra-indicated by low grade pulmonary infection and poorly controlled diabetes. This patient, nevertheless, had an excellent outcome. Comment: Amiodarone pulmonary toxicity is a rare event and needs to be considered in all patients treated with this drug. $A$ review of the literature is presented, with recommendations for screening.

\section{MALIGNANT MESOTHELIOMA IN AUSTRALIA (1945-2000)}

James Leigh

Besearch Unit, National Occupational Health and Safety Commission. Sydney 2001, and Department of Thoracic Medicine. Concord Hospital. Sydney 2139

Between 1945 and 1980, 658 (535 male, 123 female) cases of malignant mesothelioma were identified in Australia and the condition was regarded as rare.

Since 1981 Australia has maintained a complete national register for cases of malignant mesothelioma. This is a specific disease register with histologically confirmed notifications accepted from clinicians and medical records administrators. Cross checks using patient identified data are regularly carried out with all State cancer registries in Australia. Incidence has increased rapidly and is still increasing in both males and females. In 1999 the extrapolated annual incidence rate was 6.6 per 100,000 (male $>20 /$ year) (1982-85: 2.7) and 1.6 per 100,000 (female $>20 /$ year) (1982-85: 0.325). Therefore, incidence rates are similar to bladder cancer in males and uterine cancer in females. As at 3 December 1999, there were $\mathbf{5 8 0 6}$ cases in Australia, almost all of them fatal. Pleural:peritoneal ratio was found to be 18:1 (male), 6:1 (female). Positive asbestos exposure history was obtained in $85 \%$ of cases. Mean latency from first exposure was 37 years. Common exposure histories were: repair and maintenance of asbestos materials $(13 \%)$, shipbuilding $(3 \%)$, asbestos cement production $(4 \%)$, railways $(3 \%)$, power stations $(3 \%)$, boilermaking $(3 \%)$, Wittenoom ( $5 \%)$, wharf labour (2\%), paraoccupational, hobby, environmental $(4 \%)$, carpenter $(4 \%)$, builder $(6 \%)$, navy $(3 \%)$, plumber $(2 \%)$, brake linings $(2 \%)$, and multiple $(12 \%)$. The pattern of exposure is shifting away from the older traditional industries towards product, domestic and environmental exposure. The incidence is still increasing and assuming peak amphibole exposure occurred about 1965 and peak chrysotile about 1975, peak incidence is not expected until about 2010. These incidence rates are the highest national rates in the world. The estimated number of cases still to occur in Australia is about 10,000 by 2020 .

Key words: Mesothelioma, incidence, Australia, exposures

CORRECTING FOR HETEROGENEITY USING THE ILO CLASSIFICATION DOES NOT IMPROVE RADIOLOGICALPHYSIOLOGICAL CORRELATIONS

McGrath GB', Lee YC(Gary)2 ${ }^{2}$ Musk AW'.

(1) Department of Respiratory Medicine, Sir Charles Gairdner Hospital, Nedlands, WA and (2) Division of Pulmonary Medicine, St Thomas Hospital, Nashville, USA.

The International Labour Organisation classification of radiographs for the pneumoconioses is aivalidated, economical and clinically \& epidemiologically extremely useful tool in assessing for the presence and progression of asbestosis (Hilt et al 1992). The correlation between the ILO classification and functional parameters is however modest. The most densely concentrated zone determines the profusion score without regard for heterogeneity among the 6 radiographic zones thus it may not accurately represent the true disease burden. AlM: To examine the correlations between ILO score for small-opacity profusion and parameters of functional impairment (\%predicted FVC, \%predicted DLCO and \%predicted VO2max) with and without a correction for profusion heterogeneity. METHOD: Two scorers (GBM \& AWM) independently classified CXRs from 52 patients with asbestosis assigning profusion scores for each separate zone individually. The sum of the six zones' scores was averaged to provide a "corrected for heterogeneity" ILO score per radiograph. Each radiograph was also scored by conventional IL.O criteria. The "corrected" and conventional profusion scores were then tested for correlation against ventilatory function, gas exchange and exercise test results for these $\mathbf{5 2}$ subjects. RESULTS:

\begin{tabular}{|l|l|l|l|}
\hline $\mathrm{R}^{2}$ & ILO profusion & "Corrected" ILO & $\mathrm{p}^{*}$ \\
\hline \%pred FVC & 0.207 & 0.233 & $>0.4$ \\
\hline \%pred DLCO & 0.412 & 0.437 & $>0.4$ \\
\hline \%pred VO2max & 0.079 & 0.027 & $>0.4$ \\
\hline
\end{tabular}

*Using Fisher's transformation

CONCLUSION: Averaging of profusion scores for the 6 zones on the plain CXR does not improve the modest correlation between radiographs and functional parameters in patients with asbestosis. KEYWORDS: asbestosis, radiology Hilt et al, Chest Radiographs in subjects with asbestos-rolated abnormalities: comparison between ILO categorisations and clinical reading. Am J Ind Med 1992;21(6):855-61. 


\section{ENDOTOXIN AND B $(1,3)$-GLUCAN EXPOSURE IN NEW ZEALAND SAWMILL WORKERS}

Dave McLean', Jeroen Douwes1, 2, Ellen van der Maarl1, 2, Dick Heederik2, Neil Pearce'

'Wellington Asthma Research Group, Wellington School of Medicine, New Zealand 2Environmental and Occupational Health Group, Wageningen, The Netherlands

Background: Sawmill workers have been shown to have an increased risk of developing occupational asthma and other respiratory symptoms. Exposure to both wood dust and airborne microorganisms, and particularly fungal spores, has been suggested as playing a role in the development of respiratory symptoms among these workers. Few studies, however, have measured microbial exposure levels in sawmills. Methods: The preliminary study reported here assessed airborne levels of wood dust, bacterial endotoxin and $B(1,3)$-glucan in 37 personal exposure samples taken in two New Zealand sawmills. Results: The wood dust levels measured were generally low (GM 0.7 , range: $0.1-5.8 \mathrm{mg} / \mathrm{m3}$ ), with approximately $80 \%$ of the samples being below $1 \mathrm{mg} / \mathrm{m}^{3}$ and only one exceeding $5 \mathrm{mg} / \mathrm{m}^{3}$. Endotoxin levels, however, were clearly elevated above background levels (GM 68.5, range: $7-588 \mathrm{EU} / \mathrm{m}^{3}$ ) with $50 \%$ of all measured exposures exceeding $50 \mathrm{EU} / \mathrm{m}^{3}$ which is a recently recommended Dutch occupational exposure standard. The $B(1,3)$-glucan levels were comparable with levels measured in similar industries, however no quantitative dose-response relationships have been described. Dust levels were only weakly correlated with endotoxin and $B(1,3)$-glucan levels. Conclusions: Endotoxin exposures in sawmill workers are at levels sufficient to potentially contribute to the development of respiratory symptoms. Moreover, measurement of dust exposure is a poor proxy for $B(1,3)$-glucan and endotoxin exposure in sawmill workers.

Supported by the Health Research Council of New Zealand.

Key words: sawmill, wood dust, endotoxin, $B(1,3)$-glucan, asthma

\section{LUNG FUNCTION CHANGES IN ASBESTOS EXPOSED WORKERS WITH PLEURAL ABNORMALITIES}

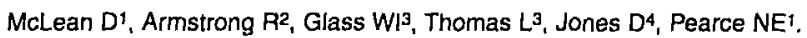

'Wellington Asthma Research Group, Wellington School of Medicine, New Zealand 2Department of Respiratory Medicine, Memorial Hospital, Hastings, New Zealand 3 Occupational Safety and Health Service, Department of Labour, New Zealand 4 Department of Respiratory Medicine, Wellington Hospital, New Zealand

Background: As asbestos exposure has declined, interest has increasingly focused on the clinical relevance of the so-called "markers of asbestos exposuren, namely pleural plaques and pleural thickening. The New Zealand Occupational Safety and Health Service maintains National Asbestos Registers, with over 800 cases on a Disease Register and over 14,000 individuals on an Exposure Register. The database includes a work history, estimated exposure index, ILO categorisation of radiographs, lung function data and basic demographic details. Methods: Cases from the Disease Register with pleural changes and with lung function measured to ATS standards, $1 \mathrm{LO}$ reading of $\mathrm{O} / 1$ or less, and with smoking and occupational data available, were selected for study. Our analysis of lung function compared actual with predicted values, and evaluated the effect of asbestos exposure and smoking habit. Results: Overall the group $(n=185)$ showed a significant loss of FEV, $(-9.11 \%$ of predicted, $\mathrm{Cl}-12.27$ to $-5.96, \mathrm{p}<0.001)$ and FVC $(-8.00 \%$ predicted, $\mathrm{Cl}-10.88$ to $-5.12, \mathrm{p}<0.001)$. FEF $25-75$ was also reduced $(-18.43 \%$ predicted, $\mathrm{Cl}-26.44$ to $-10.42, \mathrm{p}<0.001)$ where this was measured. Stratification by smoking habit showed a similar loss in FEV, and FVC in the never-smokers ( $n=52$ ). Stratification by exposure showed a clear dose response relationship in the group taken as a whole, which was maintained in never-smokers. Conclusions: Whilst preliminary, these results demonstrate an adverse effect on lung function in workers with pleural changes or thickening. These effects are independent of smoking habit, and show a clear dose response.

Presented on behalf of the National Asbestos Medical Advisory Panel. Key words: asbestos, pleural plaques, pulmonary function

\section{INHALED FINE CARBON PARTICLES ARE NOT ACUTELY BRONCHOCONSTRICTIVE.}

M.C.F.Pain', A.Hutchinson', D.Nguyen1, C. Suphioglu 2 Department of Thoracic Medicine. The Royal Melbourne Hospital and Department of Allergy. Asthma and Clinical Immunology. The Alfred Hospital2

Due to the possible association between ambient air degradation and exacerbations of asthma, we have explored the response to the inhalation of fine carbon particles. Methods. The subjects were 8 patients ( 4 males) with stable asthma (mean age $40.6 \mathrm{yrs}$ ) receiving regular inhaled corticosteroid therapy supplemented with occasional use of inhaled $\beta$-agonists. Their initial FEV 1 was $84 \%$ of predicted. Therapy was ceased at least eight hours prior to the study. All were atopic with positive skin reactions to at least three common allergens and with bronchial hyper-reactivity to lyophilised total rye grass pollen extract (mean $\mathrm{PD}_{20}=3.78 \mu \mathrm{g}$ ). Suspensions of carbon black particles in air were produced from a nebulizer/large particle trap device, the output of which produced $70 \%$ of the particles between 2.5 and 7 microns. The dust concentration was determined using a TSI Dustrak ${ }^{\mathrm{TM}}$ laser dust monitor. Single inhalations (RV to TLC) of particle suspensions of increasing concentrations, followed by breath-holding for 10 seconds were carried out and $\mathrm{FEV}_{1}$ measured at 3,10 and 20 minutes after inhalation and peak flow measurements were charted by the subjects on two occasions over the nex 4-6 hours. Most subjects received four concentrations. Results. No subjects showed any symptoms or statistically significant falls in FEV, following particle inhalation at concentrations up to $55 \mathrm{mg} / \mathrm{M}^{3}$ (equivalent to a total retained dose of approximately $100 \mu \mathrm{g}$ ). Late reactions did not occur. Conclusions. The lack of an acute bronchoconstrictive response to the inhaled dust is surprising and may have been due to the protection afforded by inhaled corticosteroid therapy or the total dose of particles delivered may be too small to elicit reactions which might be evident on more chronic exposure.

Supported by the Commonwealth Employees Medical Research Fund Key words Diesel fumes; air pollution; asthma

\section{IDIOPATHIC PULMONARY FIBROSIS - A REVIEW}

Riha, RL, Zimmerman, PV, Clarke, L, Duhig, E, Steele, $R$, Slaughter, BE The Prince Charles Hospital, Queensland, Australia

BACKGROUND: No Australian data are currently published correlating the clinical course of idiopathic fibrosing alveolitis (IPF) with histological subtype and radiological features. A review of the medical records, histology and chest radiographs of 70 cases of biopsy proven idiopathic pulmonary fibrosis was undertaken at The Prince Charles Hospital, a tertiary referral hospital for cardiothoracic disorders, from 1975 onwards. RESULTS: The median age of patients presenting with IPF was 62 years (mean $60.8+1-12.3$ yrs); $76 \%$ were male and all were Caucasian. Eleven (16\%) patients were current smokers at time of diagnosis (mean $36.6+1-11.7 \mathrm{pk} / \mathrm{yrs}$ ) and $34(48.5 \%)$ former smokers (mean $40.8+1-27$ pk/yrs). Of histological subtype, 51 (73\%) patients had a diagnosis of UIP, $5(7 \%)$ of DIP, $13(118.5 \%)$ of NSIP and one of AIP. Seven patients had dual pathologies. Dyspnoea was the primary presenting symptom in $64(91 \%)$ of patients with a median duration of symptorns of 12 months. Three patients had a family history of IPF. Open lung biopsy alone was undertaken in 32 patients, tranșbronchial biopsy alone in 18 , and both procedures in 17 . Chest radiograph was normal in $3 \%$ of cases, revealed fine nodular markings as a feature in $55 \%$ of cases, ground glass opacities in $13 \%$, ill-defined, patchy infiltrates in $26 \%$ and honeycombing in $27 \%$. No significant difference was found among histological subtypes in clinical presentation or tests of respiratory function. Steroids alone were first-line treatment in $28(40 \%)$ patients and no treatment was undertaken in $17(24 \%)$ patients. Fifteen patients received combination cytotoxic and steroid; 10 undenwent transplant assessment and one underwent lung transplantation. Median survival in patients with UIP from date of diagnosis was 17 months (range 1 - 192 months). CONCLUSION: Clinical features alone do not allow for distinction among histological subtypes of IPF. Steroids with or without a cytotoxic agent remain initial treatment options with decision to treat subject to a number of considerations. This study represents an important starting point to the prospective evaluation of IPF in Australia.

Key words: idiopathic pulmonary fibrosis 
RESPIRATORY SYMPTOMS AND LUNG FUNCTION CHANGE IN WELDERS. HOW DO THEY COMPARE TO WORKPLACE EXPOSURES?

Tania Slater ${ }^{1}$, David Fishwick ${ }^{2}$, Lisa Bradshaw ${ }^{2}$, Andrew Curran², Neil Pearce'.

1Wellington Asthma_Research Group. Wellington School of Medicine, New Sheffield, United Kingdom

The possibility was investigated that work related respiratory symptoms and acute falls in $\mathrm{FEV}_{1}$ seen in current welders relate to measured workplace exposures to total fume and metals. Methods: Changes in pulmonary function and reported respiratory symptoms were recorded in 75 welders (and non-welders) in four work sites in New Zealand. Personal breathing zone levels of total fume and various metals were also determined. Results: Work related respiratory symptoms were reported by $21.3 \%$ of all workers and related significantly only to personal breathing zone nickel exposure; (odds ratio (OR) and 95\% confidence interval (CI) of the high exposure group in relation to a low exposure group) OR 7.0, Cl 1.3-36.6. There were non signiticant associations (odds ratio $>2.5$ ) seen with total fume exposure, exposure index of greater than 10 years and age between 25 and 40 years. A fall in FEV, of at least $5 \%$ after 15 minutes of work was significantly associated with aluminium exposure alone; OR $5.8 \mathrm{Cl}$ 1.7-20.6. Conclusions: Current MIG and TIG welding, and particularly respectively nickel and aluminium exposure are associated with work related respiratory symptoms and a fall in FEV, of at least $5 \%$ after 15 minutes of work.

Supported by the Health Research Council of New Zealand.

Key words: Occupation, epidemiology, respiratory symptoms, welders.

Posters - Population Health Zealand and 2Sheffield Occupational and Environmental Lung injury Centre.

PARTICULATE AIR POLLUTION AND HOSPITALL ADMISSIONS IN CHAISTCHURCH

J. McGowan" P. Hider", E. Chacko", G. I. Town"

Department of Mathematics \& Statistics", University of Canterbury; New

Zealand Health Technology Clearing House ${ }^{+}$and Department of Medicine\#, Christchurch School of Medicine, Christchurch, New Zealand.

Aims: Winter air pollution in Christchurch is dominated by particulate from solid fuel domestic heating. The aim of the study was to explore the relationship between particulate air pollution and admissions to hospital with cardiorespiratory illnesses.

Methods: Air pollution data $\left(\mathrm{PM}_{10}\right)$ were obtained from the Canterbury Regional Council monitoring station in the central city. The New Zealand Health Information Service provided data on admissions to the Princess Margaret and Christchurch Hospitals for the period June 1988 through December 1998 for both adults and children with cardiac and respiratory disorders. The relationship between $\mathrm{PM}_{10}$ and admissions was explored using a time series analysis approach controlling for weather variables. Missing data were interpolated from carbon monoxide values for the same time period, which showed a close relationship with $\mathrm{PM}_{10}$.

Results: There was a significant association between $\mathrm{PM}_{10}$ levels and cardiorespiratory admissions. For children and adults combined there was a $2.52 \%$ increase in respiratory admissions for each interquartile rise in $\mathrm{PM}_{10}$ (interquartile value $14.8 \mathrm{mcg} / \mathrm{m}^{3}$ ). In adults there was a $1.26 \%$ rise in cardiac admissions for each interquartile rise in $\mathrm{PM}_{10}$. There was no relationship between $\mathrm{PM}_{10}$ and admissions for appendicitis, the control condition selected.

Conclusions: In keeping with international literature, there is evidence in Christchurch of a relationship between particulate levels and admissions with cardiac and respiratory illnesses. The size of the effect is in keeping with overseas data with the greatest impact seen on the respiratory system.

EXPERIENCE OF TROCAR-FREE THORACOSTOMY TUBE PLACEMENT BY NASAL SPECULUM TECHNIQUE AT THE SIR CHARLES GAIRDNER HOSPITAL

McGrath GB and Phillips MJ

Department of Respiratory Medicine, Sir Charles Gairdner Hospital, Nedlands, Western Australia.

Tube thoracostomy is commonly performed using a trocar, which provides rigidity and assists placement of the catheter tip into the desired position. Following the fatal impaling of a patient's myocardium in 1997, our hospital adopted a new technique for inserting and directing a trocar-free ICC by nasal speculum.

AIM: To examine the indications for, problems with, and patient characteristics of those requiring thoracostomy tube placement in the department over the last 2 years since the change in technique. METHODS: 32 patients who required ICC placement in clinical management were recorded on a database for analysis. AESULTS: 13 patients $(41 \%)$ had pneumothorax and 21 (66\%) had pleural effusions ( 2 had both indications). Nine $(28 \%)$ were performed emergently. The most frequent problems noted were significant pleural fluid leakage (19/21) and increased analgesia requirement (19/32). Thirty-four percent had misplacement of the catheter tip and in only 4 was the speculum too short (average BMI 30). There was low correlation between number of problems per patient and their weight $(\mathrm{r} 2=0.028)$ or $\mathrm{BMI}(\mathrm{r} 2=0,155)$. Time taken for the procedure correlated modestly with number of problems per patient $(\mathrm{r} 2=0.385)$ with the average time taken 23.7 minutes (range 5 - 60 minutes). There have been no fatalities since conversion to the new technique; however, in one patient (BMI 44.4) the procedure was abandoned for reasons of too short a speculum such that a trocar was required.

CONCLUSION: Per-speculum ICC insertion is safe but appears to create more than the expected number of non-fatal complications of excess pleural fluid leak, poor directioning of the catheter tip and increased analgesia requirement because of the larger opening needed to accommodate the speculum. The procedure also takes longer to perform; however, a learning factor may account for this.

\section{COMPLIANCE IN THE CHILDHOOD ASTHMA PREVENTION STUDY (CAPS)}

Seema Mihrshahi, Jennifer Peat for the Childhood Asthma Prevention Study Team

Clinical Epidemiology Unit, New Children's Hospital, Westmead, NSW 2145 INTRODUCTION: CAPS is a randomised controlled trial to evaluate the effectiveness of dietary modification and house dust mite allergen reduction as methods for the primary prevention of asthma. The results of the study will be compromised if compliance with either of the interventions is poor. METHODS: The dietary intervention consists of supplying margarine and oils and addition of a supplement to the child's food each day. Compliance is assessed by parents self rating and nurse rating of margarine, ofl and supplement use. The house dust mite intervention consists of washing the child's bedding every 3 months in an acaricidal wash; covering the child's mattress with an allergen impermeable mattress encasing; and removing sheepskins and soft toys. Compliance was assessed by completion of a laundry diary and by nurse observations of the presence of the impermeable mattress cover on the bed. As an objective measure, dust was collected from the child's bed and assayed for Der $\mathrm{p} 1$.

RESULTS: Table : Compliance with dietary intervention at 1 year

\begin{tabular}{|l|c|c|c|c|c|c|}
\hline $\mathrm{N}=136$ & \multicolumn{2}{|c|}{ Self reported compliance (\%) } & \multicolumn{3}{|c|}{ Nurse report of compliance (\%) } \\
\hline & $\begin{array}{c}\text { All of } \\
\text { the time }\end{array}$ & $\begin{array}{c}\text { Most of } \\
\text { the time }\end{array}$ & $\begin{array}{c}\text { Some of the } \\
\text { time or rarely }\end{array}$ & $\begin{array}{c}\text { All of the } \\
\text { time }\end{array}$ & $\begin{array}{c}\text { Most of } \\
\text { the time }\end{array}$ & $\begin{array}{c}\text { Some of the } \\
\text { time or rarely }\end{array}$ \\
\hline Margarine & 92 & 6 & 1 & 97 & 1.5 & 1.5 \\
\hline Oil & 92 & 17 & 95 & 5 & 0 \\
\hline Supplements & 42 & 41 & 17 & 42 & 43 & 15 \\
\hline
\end{tabular}

At one year, $83 \%$ of households in the active house dust mite allergen reduction group $(n=117)$ had completed the acaricide wash and $81 \%$ of children's beds had allergen impermeable mattress covers fitted. In children's beds, $58 \%$ in the active group had allergen levels below $10 \mu \mathrm{g} / \mathrm{g}$ compared with only $29 \%$ in the control group ( $p<0.001)$.

CONCLUSION: Compliance with the use of margarines and oils was high (94$96 \%$ ) and there was good agreement between self-rating and nurse rating. Compliance with supplements, laundry routines and mattress covers was lower and will need to be improved. The finding that almost twice as many children's beds in the active group had allergen levels below $10 \mu \mathrm{g} / \mathrm{g}$, is encouraging. 
PULMONARY THROMBOEMBOLISM AND FAT EMBOLISM ARE BOTH COMMON EARLY AFTER JOINT REPLACEMENT SURGERY M Peters*, L Morgan, $W$ Bruce, $H$ van der Wall. Departments of Thoracic Medicine, Orthopaedic Surgery and Nuclear Medicine. Concord Hospital, NSW Introduction. Respiratory complications are common after arthroplasty with pulmonary thromboembolic disease (PTE) and fat embolism being the most serious. It is generally thought and taught that the high risk period for PTE is 7-10 days after surgery. As fat embolism from bone marrow should contain reticulo-endothelial cells, we hypothesised that these cells would take up colloid in the lung. We conducted a scintigraphic study designed to assess the occurrence of both diseases. Methods. Patients with previous PTE were excluded. Within 48 hours of surgery, tomographic lung studies were acquired after $99 \mathrm{~m}$ Tc MAA injection. Pre and post-operative blood gases (ABG) and relevant chest radiography/CT were obtained. ABGs were analysed as the difference in alveolar-arterial oxygen gradients, pre and post-operatively $\left(\mathrm{D}_{\mathrm{A}-\mathrm{a}}\right)$. Results Forty patients were studied $(16 \mathrm{~F}, 24 \mathrm{M})$ with a mean age of $71 \mathrm{yr}$ (Range: $36-88 \mathrm{yr}$ ). Of these, 16 were hip and 24 knee arthroplasties. Lung uptake of $99 \mathrm{~m}$ Tc Colloid was present in $35 \%$ of cases (7/16 hips and 7/24 knees). Either focal or diffuse uptake was present. Diffuse uptake was associated with mottling in the lung perfusion studies. PTE was detected in 25 of $38(66 \%)$ evaluable patients. There was no significant difference in the incidence or number of segments affected, between hip and knee arthroplasty. $D_{A-a}$ was significantly higher in patients with scan evidence either of PTE ( $p<0.05)$ or fat embolism $(p<0.05)$. All patients recovered well and were free of respiratory symptoms at 6 months. Conclusion PTE immediately after arthroplasty is common and PTE should not be discounted as a clinical diagnosis in the early post-operative period. Prospective studies are needed to establish its natural course and whether intervention is of benefit. We have developed a simple test that appears able to demonstrate fat embolism to the lungs and that this is common after joint replacement surgery.

Key words Joint replacement, complications, pulmonary embolism, fat embolism, nuclear imaging

\section{GENDER, ASTHMA AND THE IMAGE}

Rhonda Hawley,

Department of Clinical Nursing, University of Sydney, 2006

The purpose of this descriptive study of the print media was to quantify the nature of the community health message and to gain an insight into how media stories on asthma are constructed. Method: Print media articles on any aspect of asthma were obtained from two media agencies, the Sydney Morning Herald and Associated Consolidated Press for the 40 month period from January 1995 to April 1998. This included daily metropolitan newspapers and non-daily print magazines available in Sydney. Content analysis was then undertaken on the news articles $(n=160)$. The news articles were coded according to which newspaper or magazine published the article, controversial issues relating to asthma (where opposing views were given), type of message (information or action orientated), spokesperson, main themes and images created. Results: The vast majority of messages gave information (81\%), both sides of controversial issues were reported and each side received approximately the same coverage. The most frequently quoted spokesperson was from the medical profession $(50 \%)$, however when sports personalities were quoted $(5.6 \%)$ they received more coverage and space. One of the main themes which emerged, was asthma across the life span; however, the images that accompanied this theme revealed a story which indicated that asthma was gendered and that this image was consistent from childhood through to early adulthood. Conclusion: Within the limits of this study the media provided an important forum for discussion and interpretation of health messages on asthma. This study also found that when a story was accompanied by a picture the image created was one that reinforced typical feminine and masculine stereotypes in Western society.
THE ASTHMA MANAGEMENT PROFILE QUESTIONNAIRE (AMPQ): A TOOL TO ASSESS PATIENT ATTITUDES TO ASTHMA, ADHERENCE AND SELF-MANAGEMENT.

FA Finlayson'1, S McLellan'2, EH Walters ${ }^{3}$, S Cronin ${ }^{4}, \mathrm{~F}$ Thien², RE O'Hehir², $J$ Douglass?

${ }^{1}$ Lung Health Promotion Centre, Departments of ${ }^{2}$ Allergy and Clinical Immunology and ${ }^{3}$ Respiratory Medicine, 4 Ward 5D, The Alfred, Melbourne, Victoria, 3181.

A questionnaire (AMPQ) was developed as part of The Alfred Inpatient Asthma Education Program (IAEP). The IAEP was developed to enable inpatients with asthma to receive an individualised education program that included consideration of health beliefs to promote positive health behaviour change and improve self-management. Aim: To develop the AMPQ as an instrument to assist the educator to explore and address attitudes to asthma .with the patient, in addition to assessing the educational needs of the person. Method: A 31-item questionnaire was devised in six sections: Health Beliefs, Causes of Asthma, Medications, Devices/ Techniques, Monitoring Asthma and Action Plan based on two previously published questionnaires. This was administered to 30 people admitted to Ward 5D at The Alfred with asthma over a two-month period. The responses to the questionnaire were summarised and nursing staff was asked to provide format evaluation on the AMPQ. Results: $19(66 \%)$ patients held health beliefs that were consistent with positive health behaviour change. $20(67 \%)$ understood what happens in the airways when they have symptoms. 12 $(40 \%)$ reported they were smokers. $14(47 \%)$ people were unsure about the role of their medications. $27(90 \%)$ had been prescribed an aerosol steroid and of these $17(58 \%)$ used a spacer. $19(70 \%)$ reported forgetting to take their preventer and $7(37 \%)$ forgot more than 6 times per week. $20(67 \%)$ had a peak flow meter. Administration time was 10-15 mins. All the nurse educators were positive about the length of time involved and the information collected. Conclusion: The Asthma Management Profile Questionnaire provided useful information to educators about patient health beliefs and asthma management. This allowed the information delivered to be tailored to meet the specific needs of the patient in the context of their health beliefs.
CASE DISCUSSION: THE IMPORTANCE OF NRESERVING AUTONOMY IN THE DYING PERSON WITH CYSTIC FIBROSIS - THE NURSE'S ROLE.

B.L. Love-Smith, F.A. Finlayson, B. Miller, E.H. Walters, J.W. Wilson, T.J. Williams. Dept. of Respiratory Medicine, The Alfred, Melbourne, Vic.

Background: Lung transplantation may allow prolongation and improved quality of life in end stage cystic fibrosis. It may, however hamper the treating team's ability to offer a consistent approach to palliative care. Objective: To describe a case that illustrates the contribution of the distinctive nursing ethic to a morally just outcome in the management of the terminal event in an adult with cystic fibrosis. Case discussion: A 59-year-old male with severe lung disease and substantially reduced quality of life was receiving inpatient treatment. The primary aim of medical management was the preservation of life to transplantation, including aggressive anti-microbial, nutritional and non-invasive ventilatory support. The patient's condition deteriorated prompting an honest and open discussion of treatment options with the patient. The goal of treatment was then changed (as a direct result of the intervention of the nurse and consistent with the expressed wishes of the patient) to ensure that suffering was minimized and dignity preserved. In the context of the caring relationship that exists between nurse and patient, the role of advocate was vital to ensuring that the wishes of the patient were reflected in the treatment goals. Conclusion: In the terminal phase, it possible, the patient should be given the opportunity to make an autonomous decision when to continue aggressive medical treatment or not. This should occur at a stage when the patient can make an informed choice about how and when they wish to die. The nurse has a pivotal role in this process. 
Posters - Sleep \& Physiology

\section{RECRUITMENT IN THE CHILDHOOD ASTHMA PREVENTION STUDY} (CAPS)

Seema Mihrshahi', Nicola Vukasin'1, Samantha Forbes', Craig Wainwright ${ }^{2}$ William Krause ${ }^{2}$ and Gabrielle Prater ${ }^{1}$ for the CAPS Team.

'Clinica! Epidemiology Unit, New Children's Hospital, Westmead, NSW 2145

2Department of Respiratory Medicine, Liverpool Hospital, Liverpool, NSW 2170

CAPS is a multicentre randomised controlled trial designed to determine whether certain interventions may reduce the incidence of asthma in children.

AIM: the aim of this sub-study is to determine the main reasons for not participating in those subjects who were eligible to participate in CAPS. METHODS: Pregnant women whose unborn children were at high risk of developing asthma were identified using a short questionnaire at the antenatal clinics of 5 hospitals in western and south-western Sydney. Subjects were excluded if they had a cat at home, lived over $30 \mathrm{~km}$ from the centre of recruitment, if the baby was delivered before 36 weeks gestation, if the baby had neonatal complications or a low birth weight. Eligible subjects were sent printed information about the study and telephoned to determine whether they wanted to participate. Their reasons for not participating in the study were recorded on the questionnaire.

RESULTS: A total of 7375 women were screened and $1462(20 \%)$ women were eligible for the study. A total of 871 women ( $60 \%$ of eligible population) declined to participate. The main reasons were: $42 \%$ were not interested or too busy to participate; $22 \%$ were ineligible for the study because they delivered early, were moving out of the area or for some other reason; $16 \%$ did not call back after three attempted contacts; $7 \%$ did not agree with the study interventions; $4 \%$ gave no reason; $3 \%$ said the study was too long; $2 \%$ cited medical reasons; $2 \%$ said family/marital problems were the main reasons they couldn't participate; and $2 \%$ cited other reasons. A total of six hundred and eighteen pregnant women ( $42 \%$ of the eligible population) have been successfully recruited into the study. CONCLUSIONS: Complexity and length of the study and amount of time available for the participants were the major factors involved in deciding not to participate in this research study. Recording the reasons for not participating in studies enabled us to have a better understanding of why people chose not to participate. This may have a bearing on the way a research project is presented to the general population and the way in which recruitment into research studies is planned.

THE INCIDENCE OF SLEEP DISORDERED BREATHING IN QUADRIPLEGICS 3 MONTHS AFTER INJURY

DJ Berlowitz, DJ Brown', DA Campbell, RJ Pierce Department of Respiratory Medicine \& Victorian Spinal Cord Service 1 . Austin \& Repatriation Medical Centre, Melbourne, Victoria, 3084 There are approximately 1500 spinal cord injured (SCl) persons in Victoria (900 quadriplegics), with most having a nearly normal life expectancy. In cross-sectional studies the prevalence of sleep disordered breathing (SDB) in quadriplegics is 2 to 5 times higher than in the general population (McEvoy, 1995; Short, 1992). The reasons for this increased prevalence are unknown. Aims The aim of this study was to investigate the evolution of SDB in the first year following acute cervical SCI. Methods Assessments will commence as soon as possible post injury ( $<48 \mathrm{hrs}$ ) and comprised serial polysomnography (Compumedics TM PS2, Abbotsford, Australia) and respiratory function testing (flow-volume loops, MIPS \& MEPS).

Assessments were performed immediately post injury, at 2 weeks, 1, 3, 6 and 12 months. Evidence of undiagnosed SDB that may have existed prior to the SCI was quantified using the Maislin et al (Sleep 1995) multivariate apnoea prediction equation (MAP). Results Subject recruitment began in December 1998 and will continue for 1 year. As of 30th September 1999, 67 patients had been admitted to the VSCS, 42 quadriplegic. Ten were excluded because they were older than 70,2 because of an associated head injury and 3 declined to participate. Three have been ventilator dependent since admission, leaving 24 in the trial. Twelve subjects (11 men, average age 35 , range 18-66 years) have been studied at 3 months. All had a restrictive ventilatory deficit (average VC $45 \%$, range $14-64 \%$ ) and 10 of the subjects have $\mathrm{SDB}(\mathrm{RDI}>5)$, despite only 2 having a pre injury MAP likelihood of $>0.5$, i.e. chance of pre-existing SDB of $75 \%(\mathrm{Cl} 70-80 \%)$. Conclusion The incidence of SDB 3 months following cervical SCl is $83 \%$, significantly higher than previously reported

Supported by Physiotherapy Research Foundation-008/98 \& NHMRC997544

Key words quadriplegia, sleep apnoea

Nomination for awards None
SURFACE TENSION OF UPPER AIRWAY MUCOSAL SURFACE LIQUID Salman Ali ${ }^{1}$, Jason P. Kirkness'2, Terence C. Amis'2, Hugo K. Christenson ${ }^{3}$, Margaret Seto-Poon', and John R.Wheatley2.

(1) School of Science, University of Western Sydney, Nepean, Parramatta, NSW 2150 (2) Department of Respiratory Medicine, Westmead Hospital and University of Sydney, Westmead NSW, 2145; and (3) Department of Applied Mathematics. The Australian National University, Canberra, ACT 0200. Surface tension (ST) properties are recognised as playing an important role in the maintenance of alveolar and small airway patency. In recent years, there has been interest in the role surface forces may play in the control of upper airway patency. This concept is particularly relevant to the control of pharyngeal patency during sleep in the obstructive sleep apnea syndrome (OSAS). While the ST of saliva samples obtained from the oral cavity have been studied extensively there is little information describing the surface forces associated with oro-pharyngeal lining liquid. There are a number of approaches to the measurement of ST, however, many require relatively large samples of the liquid under study. We have now developed an approach which utilises sma!l (microlitre) sample volumes. Methods: ST was quantified using the "pull-off" force technique ${ }^{1}$ in which $S T$ is measured as the force required to separate two curved silica discs bridged by the liquid sample. After calibration with liquids of known ST, we measured the $S T$ of $0.2 \mu \mathrm{L}$ samples of upper airway surface liquid (UASL) obtained (using $0.5 \mathrm{~mm} I D$ polyethylene tubing attached to a $1 \mathrm{~mL}$ syringe) from ten adult subjects (4 females, 6 males) and from three sites within the upper ainway. Results: There was no significant difference $(P=0.5$, ANOVA) between the ST values of UASL samples obtained from under the tongue [61.6(5.4 (SD) dynes/cm], at the oral surface of the soft palate [56.1(10.1) dynes/cm] and the posterior pharyngeal wall [58.1(7.6) dynes $/ \mathrm{cm}]$ These values are similar to published values for the ST of saliva $(\sim 57$ dynes $/ \mathrm{cm}$ ). Conclusion: We conclude that the "pull-off" force technique is suitable for measuring the ST of small samples of liquid obtained from the respiratory tract and that the ST of UASL from the oro-pharyngeal wall (site of collapse in OSAS) is equivalent to that of saliva.

(1) Christenson H.K., J. Colloid Interface Sci., 121(1):170-8, 1988

Supported by: The Garnett Passe \& Rodney Williams Memorial Foundation and the NH\&MRC

Key words: oro-pharyngeal wall collapse, upper airway patency, saliva, surface tension

Nomination for Awards: John Read Prize

CPAP IMPROVES CARDIAC FUNCTION IN PATIENTS WITH CENTRAL SLEEP APNEA AND CARDIOMYOPATHY.

K. Burgess ${ }^{123}$, P. Johnson ${ }^{2}$, J. Gehring ${ }^{2}$, L. Tyler ${ }^{2}$ and N. Berend ${ }^{3}$ Department of Critical Care. Manly Hospital ${ }^{1}$, Peninsula Private Sleep Laboratory ${ }^{2}$, University of Sydney ${ }^{3}$

Introduction: Two groups in Toronto have shown improvement in cardiac function by non invasive means using nasal CPAP therapy. Their data however have not been reproduced elsewhere. Therefore the aim of this study was to assess the effects of nasal CPAP on cardiac function in patients with cardiomyopathy and central sleep apnea (CSA). Methods: 5 adult male subjects (age: $67.8 \pm 8.7 \mathrm{yrs}, \mathrm{BMl}: 25.9 \pm 7.6 \mathrm{~kg} / \mathrm{m}^{2}($ mean $\pm S D)$ ) with cardiomyopathy and CSA (central apnea/hypopnea index >'20/hour) were studied before and after three months of nasal CPAP therapy set at a level to control any coexistent upper airway obstruction. Cardiac function (cardiac index (CI), Pulmonary Artery Occlusion Pressure (PAOP), Systematic Vascular Resistance Index (SVRI) and Pulmonary Vascular Resistance Index (PVRl) was measured invasively by pulmonary artery catheter. Extravascular lung water (EVLW) was measured by double indicator dilution technique. CPAP compliance was measured objectively by measuring actual mask application time (Respironics Aria's). In three subjects data were also collected after 12 months of CPAP treatment. Results: The mean CPAP compliance was $6.3 \pm$ 0.7 hours per night and the mean pressure was $13 \pm 2.5 \mathrm{cmH}_{2} \mathrm{O}$.

\begin{tabular}{|c|c|c|c|}
\hline Measurement & Baseline $(n=5)$ & 3 months $(n=5)$ & 12 months $(n=3)^{1}$ \\
\hline CI & $1.7 \pm 0.31 / \mathrm{min} / \mathrm{m}^{2}$ & $2.4 \pm 0.6 / / \mathrm{min} / \mathrm{m}^{2} *$ & $2.89 \pm 0.8 \mathrm{lmmin} / \mathrm{m}^{2}$ \\
\hline PAOP & $19 \pm 9 \mathrm{mmHg}$ & $16 \pm 9 \mathrm{mmHg}$ & $11 \pm 2 \mathrm{mmH}$ \\
\hline SVRI & $2980 \pm 1053 \mathrm{~d} \cdot \mathrm{cm}-5$ & $2366 \pm 966 \mathrm{~d} \cdot \mathrm{cm}-5$ & $2179 \pm 628 \mathrm{~d} \cdot \mathrm{cm}-5$ \\
\hline PVRI & $622 \pm 389 \mathrm{~d} \cdot \mathrm{cm}-5$ & $384 \pm 230 \mathrm{~d} \cdot \mathrm{cm}-5$ & $261 \pm 191 \mathrm{~d} \cdot \mathrm{cm}-5^{* *}$ \\
\hline EVLW & $10.6 \pm 2.1 \mathrm{~m} / \mathrm{kg}$ & $10.6 \pm 5.4 \mathrm{ml} / \mathrm{kg}$ & $7 \pm 1.8 \mathrm{ml} / \mathrm{kg}$ \\
\hline
\end{tabular}

Data are mean $\pm \mathrm{SD}_{1}{ }^{*}=p<0.1,{ }^{* *} p<0.05,1$ : one subject died at 6 months after stopping CPAP. Conclusion: These preliminary results show by direct measurement trends towards significant improvement in cardiac function after three months CPAP treatment. The improvement appeared to continue over the following 9 months.

Acknowledgment: ALF, NSAHS, Peninsula Private Sleep Laboratory 
NASAL CPAP IMPROVES CENTRAL SLEEP APNEA AND UNMASKS OBSTRUCTIVE SLEEP APNEA IN PATIENTS WITH CARDIOMYOPATHY.

K. Burgess 123, P. Johnson² , J. Gehring2, L. Tyler² and N. Berend ${ }^{3}$ Department of Critical Care. Manly Hospital', Peninsula Private Sleep Laboratory2 University of Sydney ${ }^{3}$

Introduction: The potential interactions between central sleep apnea (CSA), obstructive sleep apnea (OSA) and cardiac dysfunction are not well defined. Therefore the aim of the study was to assess the effect of 3 months nasal CPAP therapy on sleep disordered breathing in patients with (CSA) due to cardiomyopathy. Methods: 5 adult male subjects (age: $67.8 \pm 8.7$ yrs, BMI: $25.9 \pm 7.6 \mathrm{~kg} / \mathrm{m}^{2}$ (mean $\pm \mathrm{SD}$ )) with severe cardiomyopathy and CSA (central apnea/hypopnea index $>20 /$ /hour) were treated with nasal CPAP for 3 months after a CPAP pressure determination study. Three subjects continued CPAP for 12 months. Polysomnographic measurements were recorded after 3 and 12 months. A pulmonary artery catheter was used to measure Cardiac Index (CI) invasively. All patients received standard medical therapy which was either unchanged or reduced during CPAP therapy. Results: Patients BMI did not change. The mean compliance was $6.3 \pm 0.7$ hours per night and the mean pressure was $13 \pm 2.5 \mathrm{cmH}_{2} \mathrm{O}$.

\begin{tabular}{|c|c|c|c|}
\hline Measurement & Baseline $(\mathrm{n}=5)$ & 3 months $(\mathrm{n}=5)$ & 12 months $(\mathrm{n}=3)$ \\
\hline Arousal Index & $60 \pm 31 / \mathrm{h}$ & $41 \pm 22 / \mathrm{h}^{*}$ & $37 \pm 17 / \mathrm{h}$ \\
\hline Central RDI & $51 \pm 14 / \mathrm{h}$ & $19 \pm 20 / \mathrm{h}^{* *}$ & $1 \pm 1.2 / \mathrm{h}^{* *}$ \\
\hline Obstructive RDI & $4 \pm 5 / \mathrm{h}$ & $24 \pm 38 / \mathrm{h}$ & $38 \pm 9 / \mathrm{h} * *$ \\
\hline Total RDI & $55 \pm 1 / \mathrm{h}$ & $43 \pm 38 / \mathrm{h}$ & $39 \pm 7 / \mathrm{h}$ \\
\hline Minimum SaO & $79 \pm 10 \%$ & $80 \pm 6 \%$ & $83 \pm 8 \%$ \\
\hline $\begin{array}{c}\text { Plasma } \\
\text { Noradrenaline Night }\end{array}$ & $5.8 \pm 2.4 \mathrm{nmol} / /$ & $3.0 \pm 1.4 \mathrm{nmo} / \mathrm{n}^{* *}$ & $1.4 \pm 2.0 \mathrm{nmol} / \mathrm{h}^{* * *}$ \\
\hline Cl & & & \\
\hline
\end{tabular}

Data are mean $\pm S D,{ }^{*}=p<0.1,{ }^{* *} p<0.05,{ }^{* * *} p<0.001,1:$ one subject died at 6 months after stopping CPAP. Conclusion: Nasal CPAP dramatically improved CSA. The improvement paralleled the normalisation of nocturnal plasma noradrenaline, and improved cardiac function. Interestingly, significant obstructive sleep apnea developed (? was unmasked) in these subjects as CSA resolved.

Acknowledgment: ALF, NSAHS, Peninsula Private Sleep Laboratory

EXTERNAL NASAL DILATOR STRIPS DECREASE NASAL RESISTANCE IN OBSTRUCTIVE SLEEP APNOEA PATIENTS WITH HIGH NASAL RESISTANCE

Emily Di Somma, Stephen West, Philomena Mayrhofer, Peter Briffa, Terence C Amis, John R Wheatley.

Department of Respiratory Medicine, Westmead Hospital and University of Sydney, Westmead, NSW 2145.

The external nasal dilator strip (ENDS; Breathe Right TM, 3M Co.) is a mechanical device which has been advocated for reduction of snoring and promotion of nasal breathing. In addition, we have previously shown that ENDS reduces inspiratory nasal airflow resistance (An) by approximately $17 \%$ in healthy subjects with a normal Rn. However, there are few data available concerning quantitative effects of ENDS on Rn in disease states, such as snoring and obstructive sleep apnoea (OSA). Methods: We have now studied the effect of ENDS on inspiratory Pn in 17 patients $[11$ male; 6 lemale; age = 45 (4 SEM) years; $\mathrm{BMI}=37$ (3) $\mathrm{kg} \cdot \mathrm{m}^{-2}$ ) diagnosed with OSA (respiratory disturbance index $>10$ events/hr). Patients were selected on the basis of an abnormally high (>3.3 $\mathrm{cm} \mathrm{H}_{2} \mathrm{O} / / \mathrm{s}$ ) resting inspiratory $\mathrm{Rn}$ (at $0.4 \mathrm{l} / \mathrm{s}$ ). Using posterior rhinomanometry, inspiratory $\mathrm{Rn}$ (at $0.4 \mathrm{l} / \mathrm{s}$ ) was measured in the upright seated posture: 1) without ENDS; 2) with ENDS; and 3) without ENDS and following topical nasal decongestant (oxymetazoline hydrochloride, 0.5 $\mathrm{mg} / \mathrm{ml}$ ). Results: Inspiratory Rn decreased from $7.10(0.87) \mathrm{cm} \mathrm{H}_{2} \mathrm{O} / \mathrm{s}$ to $4.59(0.80) \mathrm{cm} \mathrm{H}_{2} \mathrm{O} / \mathrm{s}$ with ENDS, and to 2.29 (0.32) $\mathrm{cm} \mathrm{H}_{2} \mathrm{O} / \mathrm{s}$ with topical nasal decongestant (all $P<0.05$, ANOVA). Thus, in OSA patients with a high baseline Rn, ENDS reduced mean Rn by approximately $35 \%$ while topical nasal decongestant achieved an average $68 \%$ reduction in Rn. Conclusions: We conclude that while the ENDS device is effective in decreasing An in OSA patients with a high Fn, the effect is only about half that achieved using a sympathomimetic topical nasal decongestant.

Supported by: NH\&MRC

Key words: upper airway, nasal airflow resistance, OSA.

Nominations for Awards: Nil
A RESPIRATORY WARD BASED VENTILATOR WEANING PROGRAM Karen Detering David Berlowitz Linda Schachter. Department of Respiratory Medicine, Austin \& Repatriation Medical Centre, Heidelberg Victoria, Australia

The Victorian Respiratory Support Service (VRSS) was established in June 1996 to provide complex ventilatory support. This included facilitating early discharge of ventilator dependent patients from Intensive Care Units (ICU) and providing ward based weaning. Method: A 3yr retrospective audit of the VRSS records was performed. All trachesotomised patients with weaning difficulties were included. Results:

\begin{tabular}{|c|c|c|c|c|c|}
\hline & $\begin{array}{c}\text { All } \\
\text { patients }\end{array}$ & $\begin{array}{l}\text { Stable } \\
\text { NMD }\end{array}$ & $\begin{array}{l}\text { Progressive } \\
\text { NMD }\end{array}$ & COPD & Oher \\
\hline No of patients & 39 & $15(38.5 \%)$ & $4(10.3 \%)$ & $14(35.9 \%)$ & $6(15.4 \%)$ \\
\hline Age - years $\pm 95 \% \mathrm{Cl}$ & $59.0 \pm 4.4$ & $54.1 \pm 6.9$ & $48.6 \pm 21.5$ & $65.5 \pm 4.4$ & $62.7 \pm 11.5$ \\
\hline Gender (\% male) & 48.7 & 80 & 50 & 14. & 50 \\
\hline FEV1\%predicted $\pm 95 \% \mathrm{Cl}$ & $40.4 \pm 8.3$ & $45.5 \pm 14.2$ & $29.5 \pm 4.9$ & $31.9 \pm 7.4$ & $60.8 \pm 29.2$ \\
\hline FVC \%predicted $\pm 95 \% \mathrm{Cl}$ & $54.6 \pm 9.2$ & $55.0 \pm 16.0$ & $26.0 \pm 3.9$ & $56.7 \pm 14.4$ & $60.2 \pm 18.8$ \\
\hline Referral $\mathrm{PaCO} 2 \pm 95 \% \mathrm{Cl}$ & $54.7 \pm 5.6^{\circ}$ & $46.2 \pm 7.5$ & $50.1 \pm 10.9$ & $67.6 \pm+0.2$ & $48.8 \pm 5.5$ \\
\hline $\begin{array}{l}\text { Referral PaO2/FiO2 } \\
\pm 95 \% \mathrm{Cl}\end{array}$ & $\begin{array}{l}267.1 \pm \\
27.04\end{array}$ & $\begin{array}{c}263.8 \pm \\
44.2\end{array}$ & $310.3 \pm 58.9$ & $\begin{array}{c}249.5 \pm \\
39.4\end{array}$ & $\begin{array}{r}287.6 \\
+98.0\end{array}$ \\
\hline $\begin{array}{l}\text { Final } \mathrm{PaCO} 2 \\
\pm 95 \% \mathrm{Cl} \\
\end{array}$ & $48.7 \pm 3.5^{\circ}$ & $45.6 \pm 4.3$ & $40.9 \pm 12.6$ & $\begin{array}{c}54.3 \pm \\
6.5\end{array}$ & $49.4 \pm 8.4$ \\
\hline $\begin{array}{l}\text { Final PaO2/FiO2 } \\
\pm 95 \% \mathrm{C}\end{array}$ & $\begin{array}{l}321.5 \pm \\
26.7 \pm\end{array}$ & $\begin{array}{c}346.7 \pm \\
44.5\end{array}$ & $405.0 \pm 58.9$ & $\begin{array}{c}267.9 \pm \\
38.6 \\
\end{array}$ & $\begin{array}{c}319.0 \pm \\
40.9\end{array}$ \\
\hline $\begin{array}{l}\text { Compliete weaning } \\
\text { (no ventilation required) }\end{array}$ & $20(51.3 \%)$ & $40 \%$ & $0 \%$ & $72.7 \%$ & $50 \%$ \\
\hline $\begin{array}{l}\text { Partial weaning } \\
\text { (nocturnal ventilation only) }\end{array}$ & $15(38.5 \%)$ & $46.7 \%$ & $50 \%$ & $27.3 \%$ & $50 \%$ \\
\hline Tracheostomy removal & $31 / 39$ & $11 / 15$ & $2 / 4$ & $12 / 14$ & $6 / 6$ \\
\hline $\begin{array}{l}\text { Hospital LOS. } \\
\text { median(range) }\end{array}$ & $\begin{array}{c}43 \\
(6-785) \\
\end{array}$ & $\begin{array}{c}119 \\
\langle 6-412) \\
\end{array}$ & $\begin{array}{c}42.0 \\
(24-785)\end{array}$ & $\begin{array}{c}34.5 \\
(16-173)\end{array}$ & $\begin{array}{c}45.5 \\
(21-71) \\
\end{array}$ \\
\hline 12 month survival & $30 / 39$ & $14 / 15$ & $3 / 4$ & $9 / 14$ & 0 \\
\hline
\end{tabular}

* $\triangle$ Significance level of $p<0.05$. Zonclusion: Ward based weaning can be successfully performed in a range of disease processes and is associated with tracheostomy removal, improved gas exchange, and low mortality. This study was funded by the Department of Respiratory Medicine ARMC

EFFECT OF NECK POSITION, MANDIBULAR ADVANCEMENT AND MOUTH OPENING ON HYPOPHARYNGEAL DIMENSIONS Douglas JA, Noffsinger WJ, Singh B, Hiliman DR

Department of Pulmonary Physiology, Sir Charles Gairdner Hospital, Perth.

Introduction: Pharyngeal narrowing predisposes to obstruction during sleep. Aim: To determine the effects of neck position (NP), mouth opening (MO) and mandibular advancement (MA) on hypopharyngeal dimensions (antero-posterior (AP), lateral (L), cross-sectional area (XSA)). Methods: 5 healthy subjects ( $44 \pm 12$ years (mean $\pm S D)$ ) were studied awake using a naso-pharyngoscope (tip in velopharynx) to visualize the hypopharynx. They were studied at 3 levels of MA (neutral, $5 \mathrm{~mm}$, maximum $(7-10 \mathrm{~mm})$ ), 3 levels of MO (teeth opposed, $10 \mathrm{~mm}$ apart, $20 \mathrm{~mm}$ apart) and $3 \mathrm{NP}$ (neutral $\left(0^{\circ}\right.$ ), flexion $\left(-20^{\circ}\right)$, extension $\left(+20^{\circ}\right)$ ). During tidal breathing, end-inspiratory images were stored digitally. AP and $L$ dimensions were obtained from these images and expressed in units of epiglottic width to correct for magnification. XSA was calculated from AP and $\mathrm{L}$. The separate effects of changes in NP, MO and $M A$ on hypopharyngeal dimensions ( $A P, L, X S A)$ were determined relative to each baseline condition (neck neutral, teeth opposed, mandible neutral) (multiple regression).

\begin{tabular}{|l|c|c|c|c|c|c|c|c|c|}
\hline Results: & \multicolumn{3}{|c|}{ Neck Neutral } & \multicolumn{3}{|c|}{ Teeth Opposed } & \multicolumn{3}{c|}{ Mandible Neutral } \\
\hline Coeff: & $\mathrm{AP}$ & $\mathrm{L}$ & $\mathrm{XSA}$ & $\mathrm{AP}$ & $\mathrm{L}$ & $\mathrm{XSA}$ & $\mathrm{AP}$ & $\mathrm{L}$ & $\mathrm{XSA}$ \\
\hline $\mathrm{NP} \% /^{\circ}$ & $0.46^{\mathrm{d}}$ & $0.66^{\mathrm{d}}$ & $1.15^{\mathrm{d}}$ & $\mathrm{ns}$ & $\mathrm{ns}$ & $\mathrm{ns}$ & $0.19^{\mathrm{a}}$ & $0.30^{\mathrm{b}}$ & $0.51^{\mathrm{c}}$ \\
\hline $\mathrm{MO} \% / \mathrm{mm}$ & $\mathrm{ns}$ & $\mathrm{ns}$ & $\mathrm{ns}$ & $\mathrm{ns}$ & $-0.57^{\mathrm{b}}$ & $\mathrm{ns}$ & $-0.40^{\mathrm{a}}$ & $-0.72^{\mathrm{d}}$ & $-1.22^{\mathrm{d}}$ \\
\hline $\mathrm{MA} \% / \mathrm{mm}$ & $\mathrm{ns}$ & $\mathrm{ns}$ & $\mathrm{ns}$ & $\mathrm{ns}$ & $\mathrm{ns}$ & $\mathrm{ns}$ & $1.12^{\mathrm{d}}$ & $1.11^{\mathrm{d}}$ & $2.63^{\mathrm{d}}$ \\
\hline
\end{tabular}

$a=p<0.05, b=p<0.01, c=p<0.005, d=p<0.001$

Neck extension increased all dimensions. MO decreased lateral dimensions. MA increased all dimensions with this increase enhanced by neck extension but diminished by neck flexion and MO. Conclusions: Pharyngeal dimensions can be increased by MA, mouth closure and neck extension. Their study may help determine optimal mandible position for MA therapy. Neck extension may be a useful additional treatment principle. Key words: Airway dimensions, Mandibular advancement, Neck position 
NASAL POWER DYNAMICS DURING EXERCISE

J.M. Gehring, S.R. Garlick, J.R. Wheatley and T.C. Amis

Department of Respiratory Medicine. Westmead Hospital and University of Sydney, Westmead, NSW. 2145

Flow resistive work of nasal breathing (WONB) and nasal power (NP) values are known to be consistent, subject specific, predictors of the 'switching point' ventilation from nasal to oro-nasal breathing during exercise. Methods: To investigate the relationship between NP and nasal ventilation $(V)$ within an individual subject, we studied 13 healthy, adult subjects ( 6 males, 7 females; age: 27.8(6.2 SD) yrs); who performed a progressive, graded exercise task (30 to $140-230$ watts in 2 minute, 30 watt steps) on a cycle ergometer while breathing only via the nose. Tidal volume $\left(V_{T}\right)$ was monitored using an integrated nasal airflow signal obtained with a nasal mask and pneumotachograph. Trans-nasal pressure was calculated as the difference between nasal mask pressure and oro-pharyngeal pressure (measured using a stoppered mouthpiece, with no flow in a patent oral pathway). Inspiratory WONB (joules per breath, mean of 5 steady-state breaths) was measured by planimetry from pressure-volume plots constructed from the trans-nasal pressure and $V r$ data at each level of exercise. Inspiratory NP was calculated as inspiratory WONB $x$ respiratory rate (i.e. joules/sec or watts) and increased progressively with exercise. The relationship between NP and $V$ for each subject was mathematically fitted using the function $N P=a V_{0}$ (where $a$ and $b$ are constants, $R^{2}>0.91$ ). The rate of change of NP with $V$ was calculated as the first derivative of the fitted function (NPC) and the rate of change of NPC as the second derivative (NPCR). Results: There was considerable between subject variation in these parameters such that at $V=30 \mathrm{~V} / \mathrm{min}$ (i.e. within the published range for $V$ at the 'switching point') NP, NPC and NPCR ranged from 1.02 to 2.68 watts, 0.01 to 0.22 watts $1^{-1} \mathrm{~min}$ and 0.0004 to 0.01 watts $1.2 \mathrm{~min}^{2}$, respectively. Moreover, each of these parameters was positively correlated with inspiratory nasal resistance ( $R$ n at peak nasal aiflow) measured at rest $(R n=$ 1.1-12.9 $\mathrm{cm} \mathrm{H} \mathrm{H}_{2} \mathrm{O} / \mathrm{s}$; $\mathrm{R}=0.77-0.96 ; \mathrm{P}<0.002$ ). Conclusions: Thus, a higher $\mathrm{Rn}$ at rest may lead to a relatively larger increase in NP, NPC and NPCR during exercise. We conclude that resting $R n$ influences NP dynamics at higher $V$ and, thus, may be a subject specific determinant of the 'switching point' to oral breathing during exercise.

Supported by: NH\&MRC

Key words: Nasal power, oro-nasal airflow partitioning, nasal resistance Nominations for Awards: Nil

\section{A REVIEW OF HOME BIPAP FOR OBESITY-HYPOVENTILATION} SYNDROME

R.J. Hancox \& K.F. Whyte. Department of Respiratery Medicine, Green Lane Hospital. Auckland, New Zealand

Aim: To describe the characteristics and outcomes of patients treated with nocturnal bi-level positive ainway pressure ventilation (BiPAP) at home for Obesity Hypoventilation Syndrome (OHS). Methods: A case-note review of all patients treated with home BiPAP. Results: 59 patients ( 37 male) with a primary diagnosis of OHS were treated between March 1993 and November 1999. The mean (SD) age was 45 (11). Most were either Maori $(n=26)$ or Pacific islanders (27). All were extremely obese with a mean BMi of $53 \mathrm{~kg} / \mathrm{m}^{2}$ (range 37-80). 33 had been admitted acutely (16 to intensive care) with respiratory failure. Several others had had previous acute/ICU admissions. 39 patients also had obstructive sleep apnoea. 43 had right heart failure and 30 had biventricular failure. Other co-morbidity included: polycythaemia $(n=20)$, asthma/COPD (22), hypertension (21) and non-insulin dependent diabetes (14). A striking feature was a history of cellulitis (13 patients) which often precipitated the index admission. The median duration of BiPAP therapy so far is 22 months (range 1-79). The mean (SD) weight loss since presentation is $7.7 \mathrm{~kg}(19)$. Only 2 have lost sufficient weight to stop treatment. 7 patients have changed to CPAP. 5 have refused to continue treatment, 2 have been lost to follow-up and 2 are followed up elsewhere. 37 patients continue on BiPAP. Nocturnal ventilation in most patients remains sub-normal with mean morning arterial blood gases after BiPAP : $\mathrm{pCO}_{2} 7.0 \mathrm{kPa}, \mathrm{pO}_{2} 9.3 \mathrm{kPa}, \mathrm{HCO}_{3}^{-} 29.8$ $\mathrm{mmo} /$. Despite this the mortality is surprisingly low. Only 4 are known to have died, one died from renal failure and one from obesity-related cardiomyopathy. Two probably died from respiratory failure; one 8 months after refusing to continue BiPAP, the other was thought to be non-compliant.

Conclusions:OHS in New Zealand is predominantly a disease of Maori and Pacific Islanders and is associated with important co-morbidity. This descriptive study suggests that nocturnal BiPAP ventilation may improve survival.

Key words: obesity-hypoventilation, non-invasive ventilation, B.PAP

\section{BREATHING ROUTE LABILITY IN ASTHMATIC AND HEALTHY} SUBJECTS

Mervat Hallani, Terence C. Amis \& John R. Wheatley

Dept of Respiratory Medicine. Westmead Hospital \& University of Sydney, Westmead. N.S.W. 2145.

Recently, we demonstrated for the first time that asthmatic patients spontaneously breathe exclusively via the nasal route when asymptomatic but switch to oro-nasal breathing during an acute exacerbation. We have now examined the sensitivity to external inspiratory nasal loading for switching the route of breathing in asymptomatic asthmatic patients 6 females; age $=31.8(11.7(\mathrm{SD})$ yrs; FEV1 $=83(4.2) \%$ predicted), and healthy subjects (2 females, 2 males; age $=31.0(13.5)$ yrs; FEV1 $=101(14.8$ ) $\%$ predicted). Methods: Subjects breathed via a dual compartment face mask with attached nasal and oral pneumotachographs. Starting with exclusive nasal breathing, subjects inspired via a one-way valve connected to a chamber in which a constant negative pressure (inspiratory load) was maintained. Progressively increasing external loads were applied until subjects spontaneously switched to oro-nasal breathing. The external load at which this occurred was termed the 'switching load'. Standard posterior rhinomanometry was subsequently employed for measuring nasal resistance at the 'switching load'. The total nasal resistive load (NPL) was calculated as the sum of the 'switching load' resistance and nasal resistance (at $0.41 / \mathrm{sec}$ inspiratory flow). Results: The 'switching load' for the healthy subjects was -5.5 (2.1) (SEM) $\mathrm{cmH}_{2} \mathrm{O}$ and tended to be lower for asthmatic patients ( -3.5 (0.7) $\mathrm{cmH}_{2} \mathrm{O}$ ), although this difference did not achieve sigrificance $(p=0.3$, unpaired t-test). However, the NRL for healthy subjects was 6.3(2.03) $\mathrm{cmH}_{2} \mathrm{O} / 1 / \mathrm{sec}$ and did not differ from that for the asthmatic patients (6.0(1.9) $\mathrm{cmH}_{2} \mathrm{O} / / \mathrm{sec} ; \mathrm{p}=0.9$ ). Conclusion: The total NRL at which asthmatic patients switch to oro-nasal breathing is not different from that at which healthy subjects switch. Thus, asymptomatic asthmatic patients do not appear to demonstrate enhanced sensitivity to switching of breathing route in response to increased nasal loading.

Supported by: NH\&MRC \& Australian Postgraduate Award Key words: inspiratory loading, upper airway, asthma, physiology Nominations for Awards: NIL

TRANSPORT DRIVERS ARE SLEEPIER THANWORKIMG ADULTS THEIR VEHICLE ACCIDENTS ARE RELATED TO DURATION OF SLEEP AND WORK

Mark Howard1, Christopher Worsnop', Donald Campbell2, Philip Swann33, Rob Pierce' 1Department of Respiratory Medicine. Austin Repatriation_Medical Centre. 2 Clinical Epidemiology \& Health Service Evaluation Unit. Royal Melbourne Hospital. 3 Department of Road Safety VICROADS, Victoria, Australia Introduction: Fatigue contributes to a high proportion of motor vehicle accidents (MVA). We are conducting a study to assess the relationship between MVA history and sleep and work habits, excessive daytime sleepiness (EDS) and the probability of obstructive sleep apneea (OSA) in Australian transport drivers. Method: A questionnaire has been given to 255 randomly selected drivers to date. It includes age, height, weight, sleep and work habits, MVA history, the Epworth Sleepiness Scale (ESS) to measure EDS and the Multivariate Apnoea Prediction (MAP) questionnaire. The latter assesses the probability of having OSA with a respiratory disturbance index of greater than 10. Results: Most subjects were male, 184 of 185, with an average age of $42.4\{10.1$ (SD) $N=178$ )years. Mean body mass index was $27.8 \mathrm{~kg} / \mathrm{m} 2(4.2, N=181)$ and transport drivers were more likely to be overweight or obese than the Australian male population $(N=181, p<0.05)$. Relationships were evident between drivers having an MVA in the preceding three years, and hours of sleep on work days, hours worked per week and longest shift duration ( $N=141,148$ and 146 respectively, $\mathrm{p}<0.05$ for each). There was no significant relationship between MVA rates and ESS, MAP score, age or kilometers driven per year. A high proportion of drivers, $25.6 \%$ (CI 19.2 to 32.8) had an ESS of at least 10 and drivers had significantly higher ESS scores than a reference working population $(N=168, p<0.05)$. Conclusions: In this group of transport drivers MVA history was related to hours of sleep and work, but not ESS score, MAP score, age or kilometers driven per year. They were sleepier than a reference working population. Supported by AHMRF and Victorian Ministry of Roads and Ports Key words: Sleep apnoea, Sleepiness, Transport Drivers, Motor Vehicle Accidents 
METHOD FOR VALIDATING MEASUREMENTS OF TLCO AND VA Johns DP, Stephens R, Rudolph L, Jack S and Walters EH Department of Respiratory Medicine, The Alfred and Monash University Medical School, Melbourne, Victoria 3181, Australia

The carbon monoxide transfer factor (TLCO) and alveolar volume (VA) are routinely measured to assess the integrity of that part of the alveolar-capillary membrane accessible to respired gas. However, these physiological measurements are complex and there is no method available for validating the TLCO results. Aim: To develop a method for producing a known value for TLCO and VA to validate TLCO testing systems. Method: A 3.00 litre calibration syringe, 3-way tap and two gravimetric gas mixtures (BOC) containing accurately known $\mathrm{Ne}$ and $\mathrm{CO}$ concentrations were used to simulate the TLCO manoeuvre. One gas mixture ('inspired gas') contained 0.3000 $\pm 0.0015 \% \mathrm{CO}$, and $0.4991 \pm 0.0025 \% \mathrm{Ne}$; the other mixture ('alveolar gas') contained $0.1002 \pm 0.0005 \% \mathrm{CO}$ and $0.3507 \pm 0.0018 \% \mathrm{Ne}$. Theoretical TLCO and VA values were computed (target values) based on a 3.00 litre inspired volume, standardised breath-holding time of ten seconds and the accuracy range of the gas mixtures. Theoretical values were compared to results measured using a computerised TLCO system (MedGraphics Elite, MN, USA). Measured values of TLCO were standardised to a breath-hold time of ten seconds. Results: See table. The validation method has proved easy to apply

\begin{tabular}{|l|c|c|c|c|}
\hline & $\begin{array}{c}\text { Measured }(n=6) \\
\text { mean }(\mathrm{SD})\end{array}$ & $\begin{array}{c}\text { Target } \\
\text { Absolute Range }\end{array}$ & \multicolumn{2}{|c|}{ Error From Target } \\
\cline { 4 - 5 } & Absolute & Percentage \\
\hline VC L (ATPD) & $2.94(0.015)$ & 3.00 & -0.06 & -2.0 \\
\hline VA L (ATPD) & $4.08(0.04)$ & $4.02-4.11$ & 0.02 & 0.4 \\
\hline $\begin{array}{l}\text { TLCO } \\
\mathrm{mL} / \mathrm{min} / \mathrm{mmHg}\end{array}$ & $20.3(0.19)$ & $20.6-21.4$ & -0.7 & -3.3 \\
\hline
\end{tabular}

and the TLCO and VA values obtained from the MedGraphics system were reproducible. VA was found to be accurately measured but TLCO was underestimated by $0.7 \mathrm{~mL} / \mathrm{min} / \mathrm{mmHg}$. However, TLCO was within the target range when calculated using the measured inspired $\mathrm{VC}$. This method can be easily adapted to other TLCO systems including those which use $\mathrm{He}$ or methane and can provide any physiological value for VA and TLCO. Conclusion: This TLCO validation method shows promise for confirming TLCO and VA measurements.

\section{IMPULSE OSCILLOMETRY: REPEATABILITY OF INDICES OF} RESISTANCE BEFORE AND AFTER VENTOLIN.

C. Milkins, D. Smith, E.C. Smith, A.H. Kendrick.

Department of Respiratory Medicine. Bristol Royal Infirmary, Bristol, England.

Impulse oscillometry (IOS: Jaeger) is a rapid, non-invasive method of estimating airway resistance. Resistance $\left(R_{x}\right)$, measured at frequencies from $5 \mathrm{~Hz}$ to $35 \mathrm{~Hz}$, peripheral reactance $\left(X_{5}\right)$ and the respiratory impedance $\left(Z_{\mathrm{r}}\right)$ are obtained during tidal breathing. Aim: To determine the repeatability of the indices obtained from IOS in patients before and after Ventolin. Method: 23 patients (aged 41-81yrs) produced two technically acceptable estimates of the indices from IOS, spaced 2 mins apart, each estimate being recorded over $30 \mathrm{~s}-40 \mathrm{~s}$. Repeatability was reassessed $10 \mathrm{~min}$ after inhalation of $200 \mu \mathrm{g}$ of Ventolin via spacer using the one second forced expiratory volume (FEV $\left.{ }_{1}\right)$ obtained from a volume-time curve using a wedge-bellows spirometer (Vitalograph). Results: For each index, the coefficients of repeatability and precision were obtained (British Standards Institute, 1979). The results for baseline and post Ventolin are given in the table in the form (repeatability:

\begin{tabular}{|l|l|l|}
\hline & Baseline & Post Ventolin \\
\hline $\mathrm{R}_{5}$ & $1.00: 0.71$ & $0.73: 0.52$ \\
$\mathrm{R}_{10}$ & $0.59: 0.42$ & $0.59: 0.42$ \\
$\mathrm{R}_{15}$ & $0.48: 0.34$ & $0.55: 0.39$ \\
$\mathrm{R}_{20}$ & $0.41: 0.29$ & $0.49: 0.35$ \\
$\mathrm{R}_{25}$ & $0.35: 0.25$ & $0.48: 0.34$ \\
$\mathrm{R}_{35}$ & $0.29: 0.21$ & $0.42: 0.29$ \\
$\mathrm{Z}_{\mathrm{r}}$ & $1.19: 0.85$ & $0.88: 0.62$ \\
$\mathrm{X}_{5}$ & $0.74: 0.52$ & $0.55: 0.39$ \\
\hline
\end{tabular}
precision), the units being $\mathrm{kPa} / \mathrm{/s}$. Mean $( \pm S D$ ) baseline $F E V$, was $1.6 \pm 0.7 !$ FVC was $2.5 \pm 1.01$, and $F E V_{1} \% F V C$ was $65 \%$ (range $32 \%$ - $77 \%$ ). After Ventolin, FEV 1 was $1.91 \pm 0.7 l$, FVC was $2.7 \pm 0.91$, and $\mathrm{FEV}, \% \mathrm{FVC}$ was $66 \%$ (range $32 \%-88 \%$ ). From IOS, mean baseline tidal volumes were 0.90 $\pm 0.41 l$ and $0.89 \pm 0.45 l$, and were not significantly different. After Ventolin, mean tidal volumes were $0.95 \pm 0.30 l$ and $1.0 \pm 0.4 \mathrm{l}$ and were not significantly different. They were also not significantly different from baseline values. Conclusion: In these patients there is an improvement in the repeatability and precision of $R_{5}, Z_{r}$ and $X_{5}$ obtained using IOS after administration of Ventolin via spacer device.

\section{A SLOW DECLINE IN UPPER AIRWAY DILATOR MUSCLE ACTIVTY EXISTS FOLLOWING A BRIEF HYPOXIC STIMULUS}

Amy Jordan ${ }^{1,2}$, Peter Catcheside', Fergal O'Donoghue', Doug McEvoy'. 1) Sleep Disorders Unit, Repatriation General Hospital, Daw Park. SA 5041. 2) Dept. Physiology, University of Adelaide, Adelaide SA 5005 .

Following abrupt removal of a brief respiratory stimulus, there is a slow decline in ventilation back to resting levels known as ventilatory afterdischarge (VAD). VAD has been proposed to stabilise breathing patterns and has been shown to be reduced in patients with Obstructive Sleep Apnea (OSA). Theoretically, it would appear a slow decline in upper airway dilator muscle activity would be necessary to accompany the $\mathrm{VAD}$, to prevent upper airway collapse during this time. The purpose of the current study is therefore to investigate if such an upper airway afterdischarge exists and to compare its decay to that of an inspiratory pump muscle. METHODS: Healthy volunteers ( $n=7$ to date) were exposed to multiple 45 second periods of hypoxia $\left(9 \% \mathrm{O}_{2}\right.$ in $\left.\mathrm{N}_{2}\right)$ abruptly terminated with one breath of $100 \% \mathrm{O}_{2}$. Ventilatory, inspiratory diaphragmatic and genioglossal muscle responses were measured during and after this intervention. The half times of decay were calculated for each variable. RESULTS: The mean of 63 trials are reported. The half time of decay in minute ventilation, diaphragmatic and genioglossal activity was not significantly different at 9.1 (2.3 (SEM)), 8.4 (2.4) and 9.3 (2.8) seconds respectively ( $p>0.05$ ). CONCLUSIONS: Preliminary analysis of this study shows a slow decline in upper airway dilator muscle activity concurrent with the VAD, suggesting that upper airway afterdischarge exists in healthy volunteers. The time course of this decay appears to be of similar magnitude to that of VAD. If the upper airway afterdischarge is proportionately less than VAD in any situation or in any patient group, then the upper airway would theoretically be more prone to collapse. Investigation of this phenomenon in OSA patients, during sleep and between men and women is therefore important.

\section{Supported by the NHMRC}

Key words: Genioglossus, Ventilatory Afterdischarge, Obstructive Sleep Apnea, Upper Airway function, Hypoxia.

Nominations for awards: Nil

RESISTIVE LOAD DETECTION AND MAGNITUDE ESTIMATION DURING SUBMAXIMAL STEADY-STATE EXERCISE IN NORMAL SUBJECTS.

C. Deaton(2), K. Lee(2), C.J. Spokes(2), G. Laszlo(1), A.H. Kendrick(1).

1) Department of Respiratory Medicine*.,Bristol Royal Infirmary, Bristol, Engiand. 2) Department of Physiology, University of Bristol, England

Normal subjects can rate magnitudes of added resistive loads at rest and on exercise. Aim: To investigate if, during sub-maximal steady-state exercise normal subjects can estimate the magnitude of added inspiratory loads on the first or second breath after presentation and the effects of these loads on inspiratory flow. Methods: 12 normal subjects (6F: aged 20-21yr) were studied. Exercise levels of 33 and $66 \%$ of maximal ventilation $\left(V_{E}\right)$ were obtained from a maximal treadmill test (Balke protocol). At rest, at $33 \%$ and at $66 \% V_{E}$, resistors of $\left.\left.A\right) 0, B\right) 0.3, C$ ) 0.7, D) $1.3, E$ ) 2.5 and F) 5.0 $\mathrm{cmH}_{2} \mathrm{O}^{-1}$.s were each presented 10 times, for 2 breaths, in a random order. Subjects estimated the magnitude of the resistor using a digital $100 \mathrm{~mm}$ visual analogue scale (VAS). Data were compared at rest, $33 \%$ and $66 \% V_{E}$ using ANOVA and are given as mean \pm sem in the form (Rest: $33 \%: 66 \%$ ). Results: There were significant differences $(p<0.01)$ in VAS scores between exercise levels for resistors $D(13.4 \pm 2.3: 17.4 \pm 2.7: 26.6 \pm 3.5 \mathrm{~mm})$ and $F$ $(42.3 \pm 6.1: 50.4 \pm 6.7: 60.4 \pm 6.4 \mathrm{~mm})$ but not B. Flow rate $\left(\mathrm{l} . \mathrm{s}^{-1}\right)$ significantly decreased $(p<0.05)$ on the first breath after presentation for resistor $D(33 \% ; 1.0 \pm 0.04$ to $0.8 \pm 0.05: 66 \% ; 1.1 \pm 0.06$ to $0.87 \pm 0.07)$ and $F(33 \% ; 1.09 \pm 0.11$ to $0.57 \pm 0.09: 66 \% ; 1.06 \pm 0.11$ to $0.64 \pm 0.08)$. The likelihood of detecting a resistance on the first breath was greater from $0.7 \mathrm{cmH}_{2} \mathrm{O}^{-1} . \mathrm{s}$ upwards on exercise. Conclusion: in normal subjects, steady-state exercise at $33 \%$ or $66 \% V_{E}$ significantly influences estimation of the magnitude of added inspiratory resistances and decreases inspiratory flow of the first breath after presentation. The load is more likely to be detected on the first breath than the second at higher resistances.

Key words: Perception, Exercise, Normal Subjects

Key words: Aiwways Resistance, Impulse Oscillometry 
IMPULSE OSCILLOMETRY: RELATIONSHIP OF CENTRAL RESISTANCE, RESPIRATORY IMPEDANCE AND PERIPHERAL REACTANCE TO DYNAMIC LUNG VOLUMES.

C Milkins, D Smith, EC Smith, AH Kendrick.

Department of Respiratory Medicine. Bristol Royal Infirmary Bristol. England

Impulse oscillometry (IOS: Jaeger) is a rapid, non-invasive method of estimating central resistance $\left(R_{5}\right)$, peripheral reactance $\left(X_{5}\right)$ and respiratory impedance $\left(Z_{r}\right)$ at a frequency of $5 \mathrm{~Hz}$ during tidal breathing. Aim: To determine if there is a relationship between $R_{5}, X_{5}$ and $Z_{r}$ to the $1 \mathrm{~s}$ forced expiratory volume $\left(\mathrm{FEV}_{1}\right)$, forced vital capacity $(\mathrm{FVC})$ and the $\mathrm{FEV}_{1} / \mathrm{FVC}$ ratio. Methods: 76 subjects (48M: 28F) aged $16-82 \mathrm{yr}$ (mean $56.3 \mathrm{yr}$ ) were studied. All measurements were made in the upright sitting position. Estimates of $R_{5}, X_{5}$ and $Z_{r}$ were made during resting tidal breathing over a 30 s recording period. Measurements of FEV , and FVC were obtained using a wedge-bellows spirometer (Vitalograph, UK), with the highest value for each index obtained from three technically acceptable attempts being used. Relationships between each spirometric index and $R_{5}, X_{5}$ and $Z_{r}$ from IOS were obtained using regression analysis.

Results: Mean \pm SD values of FEV 1 were $1.9 \pm 0.9 l$, FVC were $2.95 \pm$ 1.1 $l$, FEV $\%$ FVC were $62.2 \pm 13.1$ (range $28 \%$ to $78 \%$ ), $R_{5}$ were $0.54 \pm$ $0.29 \mathrm{kPa} . \mathrm{l}^{-1} . \mathrm{s}, X_{5}$ were $-0.25 \pm 0.24 \mathrm{kPa} . \mathrm{l}^{-1} . \mathrm{s}$ and $Z_{r}$ were $0.59 \pm 0.37$ $k P a . l^{-1}$.s. There were significant $(p<0.001)$ linear relationships between the spirometric indices and $R_{5}: F E V_{1}=2.80-1.71 R_{5}, r^{2}=0.32: F V C=3.93-$ $1.81 R_{5}, r^{2}=0.22: F E V_{1} / N C=74-22.8 R_{51} r^{2}=0.25 ; X_{5}: F E V_{1}=2.52+$ 2.38X $\mathrm{X}_{5}, \mathrm{r}^{2}=0.38: \mathrm{FVC}=3.67+2.71 \mathrm{X}_{5}, \mathrm{r}^{2}=0.31: \mathrm{FEV}_{1}, \mathrm{NC}=70.5+28.6 \mathrm{X}_{5}$ $\mathrm{r}^{2}=0.26 ;$ and $Z_{\mathrm{r}}: \mathrm{FEV}_{1}=2.81-1.50 \mathrm{Z}_{\mathrm{r}}, \mathrm{r}^{2}=0.37: \mathrm{FVC}=3.96-1.64 \mathrm{Z}_{\mathrm{r}} \mathrm{r}^{2}=$ 0.27 : FEV $, N C=75-19.7 Z_{r}, r^{2}=0.30$. Conclusion: In these patients there are significant relationships between airway function assessed by conventional dynamic lung volume measurements and estimates of central resistance, peripheral reactance and respiratory impedance obtained from IOS.

Key words: Airways Resistance, Impulse Oscillometry

\section{(187)}

EXTRACORPOREAL MEMBRANE OXYGENATION: INDICATIONS, TRANSPORT, PREGNANCY

Paul King 1,4, Alex Rosalion ${ }^{3}$, Michael Buist ${ }^{3}$, Geoffrey Parkin' ${ }^{2}$ Nicholas Freezer 1,4, Peter Holmes 1,4 Department of Respiratory Medicine_(2) Intensive Care Unit, Monash Medical Centre, Clayton, (3) Knox Private Hospital, Wantirna, 3152 and (4) Monash University Centre for Heart and Chest Research, Clayton, Victoria, 3168

Extracorporeal Membrane Oxygenation (ECMO) is a technique that when used appropriately, is often lifesaving. We describe the first recorded case of its use in pregnancy. Case: A 29 year old woman presented to a hospital with a 2 day history of dyspnoea, malaise and fevers. She was 25 weeks pregnant and had a past history of mild asthma. A chest $X$-ray showed bilateral consolidation, and she was admitted with a diagnosis of pneumonia and asthma. She deteriorated rapidly over the next day and had to be intubated. Despite full mechanical ventilation and paralysis her oxygen saturations were $<80 \%$. It was felt that she should be transterred to a tertiary hospital for specialised obstetric care. To facilitate her transfer and improve her oxygenation it was decided that she should be commenced on ECMO. This was done and within 10 minutes her saturations had improved remarkably to $99 \%$. She was transferred without incident. Her condition stabilised and she was weaned off ECMO after 3 days and extubated several days later. She went into spontaneous labour at 30 weeks gestation. 3 months later mother and baby are in good health with no residual problems. Discussion: ECMO has been used for almost 30 years. It has been used extensively in the treatment of respiratory failure in neonates, with survival rates of $>80 \%$. In adults it has not had such a good reputation, and until recently has been regarded as being fairly unsuccessful, with survival rates of $<10 \%$. However, improved techniques and in particular better patient selection, have resulted in recent survival figures being reported as $60-70 \%$. ECMO should be considered for young patients with acute reversible respiratory failure who have a $90 \%$ mortality risk with conventional treatment, as calculated by pulmonary compliance and vascular shunt measures. Its use in this case saved two young lives. ECMO is also a way of guaranteeing oxygenation when transferring highly unstable hypoxic patients. Several small studies have reported uniform success in transporting such patients. Portable machines are available that will itt inside an ambulance. There have been reports of ECMO in post partum patients but this is the first documented case of its use in pregnancy.

Key words: ECMO, pregnancy, respiratory failure, transport

\section{ADAPTATION TO RESISTIVE LOADS DURING SUBMAXIMAL STEADY- STATE EXERCISE IN NORMAL SUBJECTS.}

C. Deaton (2), K. Lee (2), C.J. Spokes (2), G. Laszlo (1), A.H. Kendrick (1)

1) Department of Respiratory Medicine. Bristol Royal Infirmary. Bristol. England. 2) Department of Physiology University of Bristol. England

We have observed that inspiratory flow significantly decreases on the first breath after the presentation of a resistor. The number of breaths needed to adapt to the presence of a resistor at different workloads has not been studied. Aim: To investigate if, during sub-maximal steady-state exercise, inspiratory flow and pressure returns to pre-load values within 5 breaths of presentation. Methods: 6 normal subjects (3F: aged 21-22yr). An exercise level of $33 \%$ of maximal ventilation, obtained from a maximal treadmill test, was used and resistors of A) $0, B$ ) 0.3, C) 0.7, D) 1.3, E) 2.5 and F) $5.0 \mathrm{cmH}_{2} \mathrm{O}^{-1}$.s were each presented 10 times, for 5 breaths, in a random order. Inspiratory flow (I.s-1) and mouth pressure $\left(\mathrm{cmH}_{2} \mathrm{O}\right)$ were recorded throughout. Results: There were no significant effects on flow or pressure with resistors $A$ or $B$. For resistors $C$ to $F$, inspiratory flow significantly decreased $(p<0.01)$ on the first breath after presentation and had not returned to baseline after 5 breaths. Inspiratory pressure significantly increased $(p<0.001)$ on the first breath after presentation and remained significantly greater than baseline after 5 breaths.

\begin{tabular}{|l|c|c|c|c|c|c|}
\hline Breath & Pre & Post - 1 & Post - & Post - 3 & Post - 4 & Post - 5 \\
\hline Flow & $1.1 \pm 0.1$ & $0.8 \pm 0.1$ & $0.8 \pm 0.1$ & $0.8 \pm 0.1$ & $0.9 \pm 0.1$ & $0.9 \pm 0.1$ \\
\hline Pressure & $2.6 \pm 0.4$ & $5.3 \pm 0.3$ & $5.1 \pm 0.2$ & $4.8 \pm 0.3$ & $4.4 \pm 0.3$ & $3.9 \pm 0.3$ \\
\hline
\end{tabular}

Data are shown for resistor $D$ as mean \pm sem. Conclusion: In normal subjects, inspiratory resistors of $0.7 \mathrm{cmH}_{2} \mathrm{O}$ and above significantly influence the inspiratory pressure and flow during steady-state exercise at $33 \%$ maximal ventilation. These changes indicate that adaptation, mainly through changes in inspiratory pressure occur in order to maintain an adequate tidal volume.

Key words: Perception, Exercise, Normal subjects

\section{HYPERTENSION IN OSAS: CHANGES IN THE FIRING PATTERN OF SINGLE MUSCLE VASOCONSTRICTOR NEURONES IN AWAKE PATIENTS}

V.G. Macefield, E. Balong-e', D.K. McKenzie' \& M. Elam²

Prince of Wales Medical Research Institute, Randwick, NSW 2031, Australia; 'Department of Respiratory Medicine, Prince of Wales Hospital, Randwick, NSW 2031, Australia; Institute for Clinical Neuroscience, Sahlgren University Hospital, S-413 45 Göteborg, Sweden.

Muscle sympathetic outtlow is greatly elevated in awake patients suffering from the obstructive sleep apnoea syndrome (OSAS), with the increase in multi-unit burst activity being similar to that seen in congestive heart failure (CHF). To increase our understanding of the neural mechanisms of sympathoexcitation, single-unit activity of 12 muscle vasoconstrictor neurones (recorded via tungsten microelectrodes in the peroneal nerves of 6 OSAS patients) was compared with that of 16 neurones recorded in $8 \mathrm{CHF}$ patients and 33 neurones recorded in 14 healthy controls (CTL). For both groups of patients the mean firing rates and firing probabilities were significantly higher than in the controls (Table). However, while the percentages of cardiac intervals in which neurones generated $1,2,3$ or 4 spikes were statistically identical in CHF and CTL, the OSAS patients generated significantly fewer solitary spikes and more double spikes. These firing properties were identical to those generated by 9 neurones during acute increases in sympathetic drive (inspiratory-capacity apnoea) in 5 controls. We conclude that the sympathoexcitation associated with OSAS differs from that of $\mathrm{CHF}$, perhaps due to an acute sympathetic drive related to the resting hypopnoea in OSAS.

$\begin{array}{lllll} & \text { CTL rest } & \text { CHF rest } & \text { OSAS rest } & \text { CTL apnoea } \\ \text { frequency } & 0.40 \mathrm{~Hz} & 0.98 \mathrm{~Hz}^{*} & 0.96 \mathrm{~Hz} & 1.04 \mathrm{~Hz} \\ \text { firing prob. } & 30.8 \% & 55.1 \% \%^{*} & 51.8 \% & 56.3 \% \\ 1 \text { spike } & 72.7 \% & 70.6 \% & 58.8 \% \%^{*} & 61.2 \% \\ 2 \text { spikes } & 18.4 \% & 18.2 \% & 27.3 \%{ }^{*} & 26.7 \% \\ \text { 3 spikes } & 5.1 \% & 7.3 \% & 9.7 \% & 9.5 \% \\ 4 \text { spikes } & 2.9 \% & 3.0 \% & 2.9 \% & 1.7 \% \quad("=P<0.05)\end{array}$


SURVEY OF VICTORIAN SLEEP LABORATORIES: EQUIPMENT, STAGING AND SCORING CRITERIA.

R. Manser1, P. Rochford2, T. Roebuck ${ }^{3}$, R. Pierce'2, M. Naughton ${ }^{3}$, D. Campbel/1 (1) Clinical Epidemiology Unit. Royal Melbourne Hospital. Parkville, Victoria, 3050 (2) Department of Respiratory Medicine. Austin \& Repatriation Medical Centre, Heidelberg. Victoria, 3081 (3) Respiratory Medicine, Alfred Hospital, Prahran. Victoria, 3181.

Surveys of laboratories overseas have documented significant diversity in the working definitions used for reporting respiratory events in sleep studies. Aim: To assess sources of variability in the measurement of sleep disordered breathing (as defined by the Respiratory Disturbance Index) between different sleep laboratories throughout Victoria. Methods: A selfcomplete written questionnaire was constructed following literature review and interviews with staff at 3 separate sleep laboratories. The survey was sent to all laboratories listed in Victoria by the Australasian Sleep Association in 1999. The first part of the survey related to the type of equipment used to record sleep \& other variables during overnight polysomnography and the second part to the definitions and methods used for the reporting of the results. Results: The response rate was $94 \%$ (17 out of 18 laboratories returned the surveys). Overall there was reasonable consistency between laboratories with respect to the types of variables measured during overnight polysomnography $\&$ the methods used to measure them. Some differences are described. In relation to the methods used for scoring sleep study results the greatest inconsistencies were noted in the definition of hypopnoeas. Variability was also noted in the scoring of apnoeas, \& arousals. Conclusions: Several sources of variability in the methods used to measure \& define sleep-disordered breathing are described. The extent to which these variations may affect the comparability of reported results between different laboratories requires further research. Supported by: Victorian Department of Human Services tor the CPAP Service Development Project.

Key words: Survey, sleep disordered breathing, sleep laboratories

\section{OUTCOMES OF THE VICTORIAN CPAP PILOT PROGRAM PATIENT} FOCUS GROUPS.

R. Manser', H. Teichtahl'2, M. Ho ${ }^{3}$, A. Sasse ${ }^{4}$, G. Cherry'2, J. Copland ${ }^{3}$, D. Campbell1 (1) Clinical Epidemiology Unit, Royal Melbourne Hospital, Parkvilie, Victoria, 3050, (2) Department of Respiratory Medicine. Western Hospital, Footscray, Viçtoria (3) Monash SSleep Centre. Monash Meedical Centre. Clayton, Victoria (4) Traralgon Hospital, Traralgon, Victoria.

The Victorian CPAP Pilot Program was developed to provide CPAP Services to eligible participants with sleep disordered breathing. As part of the evaluation of that program patient focus groups were conducted at institutions involved in service delivery. Aim: To examine patient perceptions of the CPAP Pilot Program with an emphasis on service delivery, outcome, side effects, reasons for presentation \& barriers to compliance. The purpose was to inform the development of a patient satisfaction survey \& validate the researchers' understanding of the research agenda from the viewpoint of patients. Methods: Focus groups were conducted by an independent observer at 4 institutions involving a total of 24 patients. Both compliant \& non-compliant patients attended. The procedure for the conduct of focus groups followed standard recommendations. Taped discussions were transcribed verbatim and the text was submitted to independent thematic analysis. Results: Most participants were happy with the delivery of the program \& its outcomes. The main area of concern to participants was the lack of technical support related to the use of equipment particularly masks, $\&$ costs related to consumables. Several triggering factors for entry to the program were identified and include; a bad driving experience, chronic tiredness \& family pressure to attend. Conclusions: The results have been used to develop a survey that will assess patient satisfaction quantitatively. Further research may help to establish whether additional technical support improves long-term adherence \& examine the relationship between triggering factors for presentation, outcome \& compliance. Supported by: Victorian DHS for the CPAP Service Development Project. Key words: CPAP, focus groups, sleep disordered breathing
MANDIBULAR ADVANCEMENT SPLINT IMPROVES INDICES OF OBSTRUCTIVE SLEEP APNOEA AND SNORING BUT SIDE EFFECTS COMMON.

Neill AM, Whyman R, Jeffery O, Bannan SPT, Campbell AJ. WellSleep Centre, Wellington School of Medicine.

OBJECTIVE: To assess the efficacy of a Mandibular Advancement Splint (MAS) in the treatment of Obstructive Sleep Apnoea Syndrome (OSAS). DESIGN: Randomized double blind crossover study.

METHODS: 18 OSAS patients, consecutively treated with a MAS, were randomised to sleep a half night with and a half night without the MAS. A paired student $t$ test was used to compare polysomnographic indices of sleep-disordered breathing and sound (Rion - integrating sound meter). Snores were scored when inspiratory noise was greater than five $\mathrm{dB}$ above background. A questionnaire was used to assess symptoms and side effects. RESULTS: 18 patients (17 male and 1 female) with a mean \pm SD age of $47.2 \pm 9.0$ years, Body Mass Index of $32.1 \pm 4.9 \mathrm{~kg} / \mathrm{m}^{2}$ and an initial Respiratory Disturbance Index (RDI) of $32.7 \pm 24.5$ /hour were studied. Total sleep time, sleep efficiency, \% REM sieep and \% sleep spent supine were similar $(p>0.05)$ with and without the MAS. The use of the MAS significantly reduced mean RDI from $22.8 \pm 20.8$ to $17.4 \pm 21.7$ /hour $(p=0.047)$, Arousal Index from $25 \pm 17$ to $19 \pm 15$ /hour $(p=0.02)$, average snoring intensity from $52.8 \pm 4.1$ to $50.9 \pm 2.7 \mathrm{~dB}(p=0.02)$. Treatment over 9.3 $( \pm 9.0)$ weeks with the MAS was associated with a small improvement in daytime somnolence (mean Epworth Sleepiness Score $12.0 \pm 4.9$ c.f. $9.9 \pm 3.1, p=0.07$ ) and significant side effects. 15 patients $(83 \%)$ reported difficulty using the MAS and $38 \%$ use the device for less than 3 nights a week.

CONCLUSION: The use of the MAS resulted in a small but statistically significant reduction in indices of OSAS. A significant number of patients had difficulty tolerating the device with $38 \%$ failing to achieve satisfactory compliance.

Supported by an Otago Research Grant
EFFECTS OF LONG-TERM NOCTURNAL NON-INVASIVE VENTILATION (NIV) ON GAS EXCHANGE IN PATIENTS WITH CHRONIC OBSTRUCTIVE PULMONARY DISEASE (COPD) AND HYPERCAPNIA.

TD Robinson, ER Collins, CE Sullivan and IH Young Department of Respiratory Medicine, Royal Prince Alfred Hospital. Sydney, NSW, 2213

It is postulated that long-term nocturnal non-invasive ventilation (NIV) reverses hypercapnic respiratory failure due to obesity hypoventilation or chest wall abnormalities largely by increasing alveolar ventilation. The effects of long-term NIV in patients with hypercapnia due to chronic obstructive pulmonary disease (COPD) remain unclear. We have performed detailed gas exchange studies using the multiple inert gas elimination technique (MIGET) on 4 patients with COPD and hypercapnic hypoxic respiratory failure, at baseline and following three months of NIV plus oxygen. All patients had severe COPD with an $\mathrm{FEV}_{1} 0.58(0.20 \mathrm{~L}$ (SD), $(21$ (6\% predicted), FVC $1.89(0.48 \mathrm{~L}$ (56(5\% predicted) and RV 221(45\% and chronic respiratory failure with $\mathrm{PaCO}_{2} 61\left(9 \mathrm{mmHg}\right.$ and $\mathrm{PaO}_{2} / \mathrm{FIO}_{2} 348(114$. At baseline, the MIGET measure of perfusion to low $V_{A} / Q$ units (log SD $Q$ ) was abnormal at $0.63(0.16$ (normal $<0.6$ ) and the MIGET measure of ventilation to high $V_{A} / Q$ units $(\log S D V)$ was abnormal at $0.76(0.31$. There was minimal shunt $(0.8(0.3 \%)$ and alveolar ventilation was $4.1(1.8 \mathrm{~L} / \mathrm{min}$ (dead space of $56(5 \%)$. After three months of NIV plus oxygen, $\mathrm{PaCO}_{2}$ fell by $>6 \mathrm{mmHg}$ in three patients and rose by $5 \mathrm{mmHg}$ in one patient with a mean $\mathrm{PaCO}_{2}$ of $54(5 \mathrm{mmHg}$ ( $p=0.1$, paired $\mathrm{t}$-test). Alveolar ventilation fell in all patients to a mean $3.7(1.8 \mathrm{~L} / \mathrm{min}(p=0.03$, paired t-test), with no change in dead space or $\mathrm{PaO}_{2} / \mathrm{FIO}_{2}$ and no significant change in log SD Q or log SD V. These findings show that improvements in $\mathrm{PaCO}_{2}$ occur with NIV despite falls in daytime alveolar ventilation. In the absence of significant changes in intrapulmonary gas exchange this suggests that the fall in $\mathrm{PaCO}_{2}$ may be due to a reduction in $\mathrm{CO}_{2}$ production, possibly due to reduced respiratory muscle work.

Supported by the NH\&MRC

Key words: COPD, MIGET, hypercapnia, non-invasive ventilation Nominations for Awards: TSANZ/ALF John Read Prize Physiological Research 


\section{MEASUREMENTS OF SMALL AIRWAYS NARROWING IN PATIENTS} WITH OBSTRUCTIVE SLEEP APNEA

Elizabeth Veitch', David Schembri', Alan Crockett² \& R.Doug McEvoy'.

(1) Respiratory and Sleep Disorders Unit, Repatriation General Hospital, Daw Park, SA, 5041. (2) Respiratory Unit, Flinders Medical Centre, Bedford Park, SA, 5042

OSA has been thought to selectively involve the pharynx or upper ainway, however several recent studies suggest that OSA may be associated with intrapulmonary airways dysfunction. If proven this would shed new light on the possible pathogenesis of airways disease and suggest a new approach to management. Aim: To investigate the presence of small airways narrowing in patients with documented OSA. Methods: Consecutive patients referred to the respiratory laboratory at RGH with a diagnosis of possible OSA underwent full respiratory function testing (RFT) and impulse oscillometry (IOS) the morning after their polysomnography(PSG). Results: 40 patients were studied with RFT, IOS and PSG (33 males). The mean age was 55 years (range 22-75) and body mass index (BMI) was 35 (range $25-50) .10(25 \%)$ patients had a history of prior or current respiratory disease (6 asthma, 4 COPD) and $28(76 \%)$ had a history of prior or current smoking. The mean RDI of the total group was 41 (range 2-112), $16(40 \%)$ patients had severe OSA (RDI>40). PSG excluded the diagnosis of OSA in only 3 patients $(\mathrm{RDI}<5)$. Significant associations with the severity of OSA (based on RDI and minimum $\mathrm{SaO}_{2}$ ) were seen with BMI $(p<0.01)$ but not with age, history of respiratory disease or smoking. Measurements of small airway narrowing by $I O S$ and flow volume curves were not significantly associated with severity of OSA based on RDI. Conclusions: Our preliminary RFT and IOS data failed to confirm the observations by other groups of abnormalities in the small airways in patients with OSA. Larger studies including larger numbers of normal (non-OSA) patients are required to further investigate this area and provide conclusive findings.

Key words: Obstructive sleep apnea, small airways, oscillometry.

EXAMINING THE ASSUMPTIONS IN A METHOD FOR MEASURING THE VOLUME DISPLACED BY DIAPHRAGM MOTION (AVdi)

B. Singh 12,2 J.A. Panizza1, K.E. Finucane ${ }^{1}$.

Departments of Pulmonary Physiology, Sir Charles Gairdner Hospital

(SCGH), Western Australia and 2Physiology, University of Western Australia. We have proposed a method for measuring $\Delta V d i$ from the change in subphrenic volume (Singh et al AJRCCM 1999;160:1507-15). The subphrenum was defined by the diaphragm dome, diaphragm-apposed rib cage and a horizontal plane at the inferior insertion of the diaphragm.

Subphrenic volume was calculated by dividing it into multiple $1 \mathrm{~cm}$ horizontal slices, the cross-sectional (CS) areas of which were determined from a geometric model of each slice and measurements from PA and lateral CXRs, and was corrected for spinal volume. In this study, we examined in healthy ( $N$ ) and hyperinflated $(H)$ subjects 1 ) the accuracy of several geometric models used to calculate lower rib cage CS area, and 2) the internal consistency of the proposed method for measuring $\triangle V$ di. The former was studied near TLC using chest CTs in $25 \mathrm{~N}$ and $22 \mathrm{H}$ subjects by comparing lower rib cage $\mathrm{CS}$ area measured by planimetry against that calculated from the mid sagittal and coronal axes of the lower rib cage using several models: ellipse, rectangle, $1 / 3$ the way between an ellipse and a rectangle, circle, and a rectangle bounded by two semicircles. The internal consistency of $\Delta V$ di was examined in $10 \mathrm{~N}$ and $9 \mathrm{H}$ subjects by comparing it with the difference between change in lung volume $\left(\Delta V_{L}\right)$ measured by the method of Pierce et al. (Thorax 1979;34:72634) and the change in lung volume attributable to lateral expansion of the rib cage $(\Delta \mathrm{Vrc})$ measured radiographically for breaths from RV to FRC, FRC $+1 / 2$ inspiratory capacity and TLC. We found that in both groups, lower rib cage CS area measured by planimeter was most closely predicted by modelling the rib cage as $1 / 3$ the way between an ellipse and a rectangle $\left(r^{2}=0.99\right.$, difference [mean $\pm \mathrm{SD}$ ] $1.3 \pm 4.2 \%$ ), and there was a close relationship between $\Delta V \mathrm{di}$ and $\Delta V_{L}-\Delta \operatorname{Vrc}\left(r^{2}=0.97\right.$, difference $\left.94 \pm 148 \mathrm{mls}\right)$. We conclude that in healthy and hyperinflated subjects, lower rib cage CS shape is close to $1 / 3$ of the way between an ellipse and a rectangle, and the proposed method for measuring $\Delta V$ di is internally consistent.

Supported by NHMRC, Medical Research Fund of WA and SCGH Research Fund.

\section{UTILITY OF AWAKE IMPULSE OSCILLOMETRY IN DETECTING AIRWAY} NARROWING IN PATIENTS WITH OBSTRUCTIVE SLEEP APNEA

Elizabeth Veitch', David Schembri', Alan Crockett² \& R.Doug McEvoy'.

(1) Respiratory and Sleep Disorders Unit, Repatriation General Hospital, Daw Park, SA, 5041 and (2) Respiratory Unit, Flinders Medical Centre, Bedford Park, SA, 5042

The "gold standard" investigation for obstructive sleep apnea (OSA) is polysomnography (PSG) which is costly and time-consuming for patients and health care staff. History and examination show poor predictive values for PSG-proven OSA and thus other investigations (cephalometry and CT scanning) have been examined for their ability to detect upper airway narrowing and consequently select those patients likely to have OSA and warranting PSG. Aim: To investigate the utility of IOS measurements in assessing upper airway resistance in awake patients with OSA. Methods: Consecutive patients referred to the respiratory laboratory at RGH with a diagnosis of possible OSA underwent full respiratory function testing (RFT) and impulse oscillometry (IOS). IOS recorded "central" and "peripheral" resistance. The former is thought to mainly reflect upper aiway resistance. Results: 40 patients were studied with RFT, IOS and PSG (33 males). The mean age was 55 years (range 22-75) and body mass index (BMI) was 35 (range 25-50). PSG excluded the diagnosis of OSA in only 3 patients (RDl<5/hr of sleep). The mean RDI of the total group was 41 (range 2-112), $16(40 \%)$ patients had severe OSA (RDI>40). There was no significant association of upper airway resistance as measured by IOS (sitting or supine) with the severity of OSA as measured by RDI. However, the small numbers of normal (non-OSA) patients prevented a meaningful comparison of upper airway resistance (as measured by IOS) between normals and OSA patients. Conclusions: Although current data do not show an association between upper airway resistance and severity of OSA our work to date provides a preliminary profile of resistance measures (by IOS) in patients with OSA.

Key words: Upper airways resistance, obstructive sleep apnea, oscillometry.

INFLUENCE OF EXPIRATORY FLOW ON ANATOMICAL DEAD SPACE MEASUREMENTS

DM Smallwood, T Demis, MCF Pain

Department of Thoracic Medicine, Royal Melbourne Hospital, Parkville. Victoria

Background. The anatomical dead space (VD) represents the luminal volume of the conducting airways not involved in gas exchange. Its measurement, using a single-breath nitrogen washout technique, is influenced by initial lung volume and the pause between inspiration and expiration of the test gas. It is unclear whether the flow rate of the expired gas influences VD. Aim. To measure the VD at varying expiratory flow rates on different days in normal subjects and determine whether a suitable range could be found that would provide consistent VD values.

Methods. 2 male subjects were recruited, aged 42(TD) and 29(PW). They had no history of respiratory disease. A modified Fowler's method utilised a single-breath nitrogen wash out after oxygen inhalation to measure VD. Subjects exhaled to FRC then inhaled $1 L$ of $100 \%$ oxygen from a reservoir bag at about $0.5 \mathrm{~L} / \mathrm{sec}$. After an inspiratory pause of less than 1 second the expired volume and nitrogen concentration were simultaneously recorded. The VD was taken as the volume that coincided with the arithmetic mean of the phase II slope of the nitrogen washout curve. The procedure was repeated at varying expiratory flow rates on 4 different days. Results. Subject TD had 63 readings taken with an expiratory flow varying from $253 \mathrm{ml} / \mathrm{sec}$ to $1278 \mathrm{ml} / \mathrm{sec}$. VD ranged from $188-372 \mathrm{ml}$. PW had 52 readings with flow varying from $264-1231 \mathrm{ml} / \mathrm{sec}$. VD ranged from $191-396 \mathrm{ml}$. VD did correlate with expiratory flow in both subjects $\left(R^{2} 0.82\right.$ for TD, $R^{2 \cdot} 0.90$ for $P W)$. VD values between flows of $350-700 \mathrm{ml} / \mathrm{sec}$ were relatively consistent. Over the 4 days TD averaged $255 \mathrm{ml}$ (range of averages 249-262), coefficient of variation $9.9 \%$. PW averaged $242 \mathrm{ml}$ (233-250), coefficient of variation $8.8 \%$. Conclusion. Expiratory flow rates do alter VD and need to be controlled. The lower VD at slow flow rates probably results from increased time for mixing at the interface. The larger VD at higher flows is probably artifactual. Using the arithmetic mean for VD calculation may introduce error. The consistent VD value at intermediate flow rates enables comparison of VD at different lung volumes.

Key words. Anatomical dead space, single breath nitrogen test Supported by the RMH Research Foundation 
SPIROMETRY IS LESS DEMANDING FOR PATIENTS AND MORE

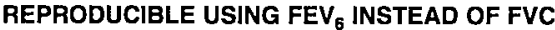

Maureen Swanney', Robert Jensen², David Crichton'1, Lutz Beckert' ${ }^{1}$, Lauren Cardno ${ }^{1}$, Robert Crapo ${ }^{2}$

(1) Respiratery Physiology Laboratory, Christchurch Hospital, Canterbury Health Ltd, Christchurch, NZ.

(2) Pu!monary Division, \& Department of Medicine, LDS Hospital \&

University of Utah School of Medicine, Salt Lake City, Utah, USA

Spirometry can be a physically demanding manoeuvre for patients with severe respiratory disease because of the long exhalation time required to meet standardised testing criteria. Aims: To determine if the forced expiratory volume in 6 seconds $\left(\mathrm{FEV}_{6}\right)$ could serve as an acceptable alternative to $\mathrm{FVC}$ in the diagnosis of airway obstruction and lung restriction.

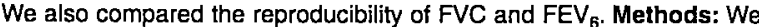
analysed data from consecutive patients referred to our laboratory for spirometry. FEV $1 / F V C$ was compared with $F E V_{1} / F V_{6}$ and $F_{E V}$ with FVC. Results: Compared to $F E V_{1} / F V C$ using $\mathrm{FEV}_{1} / \mathrm{FEV}_{6}$ the sensitivity for obstruction was $94.6 \%$ and for restriction $92.6 \%$; the specificity for obstruction was $97.4 \%$ and for restriction $100 \%$ The intra-subject coefficient of variation was 3.4 for $F V_{6} \& 4.5 \%$ for FVC. Conclusions: $F_{E V}$ is more reproducible than FVC and is an acceptable alternative to FVC for diagnosing airway obstruction. $\mathrm{FEV}_{6}$ is a good spirometric predictor of a restrictive pattern.

Key words: Spirometry, reproducibility, obstruction, restriction.

Nomination for Awards: Nil.

A STUDY OF THE EFFECTIVENESS OF LOCAL ANGEL (LOCAL ANAESTHETIC GEL) IN THE REDUCTION OF PAIN DURING ARTERIAL PUNCTURES

Ngoc Tran, Linda Schachter, Christopher Worsnop.

\section{Department of Respiratory Medicine. Austin and Repatriation Medica} Centre Victoria. 3084.

Background: Needle punctures have been shown to cause considerable pain and discomfort. Topical use of local anaesthetics has been shown to reduce the pain associated with venous punctures, especially in children. No data are yet available concerning the use of local anaesthetic with arterial punctures.

Aim: To evaluate the effectiveness of local anaesthetic gel, containing amethocaine $4 \%$ (Local AnGel), in the reduction of pain during arterial punctures.

Methods: A placebo controlled, double blinded study was employed. Gel was applied for 30 minutes prior to the procedure. Pain was rated by a visual analogue scale and a short questionnaire. The scale was from 0 (no pain) to $100 \mathrm{~mm}$ (most severe pain). Follow-up by telephone was used to determine side effects. Heart rate was monitored with a pulse oximeter. Results: Preliminary results: 32 subjects were evaluated, $19 \mathrm{M}$ and $13 \mathrm{~F}$; mean (sd) age 67.3 (12.8). 16 received placebo and 16 Local AnGel. Mean pain scores were $16.2(13.6)$ and $17.9(25)$ respectively. $(p=0.8)$. The mean (sd) heart rates before arterial puncture were 88 (11.5) with placebo and 85 (13.2) with AnGel $(p=0.5)$, and after the puncture were $89(13.4)$ and $84(13.6)$ respectively $(p=0.4)$. Twelve subjects in each group said that they would prefer to have gel applied next time they had an arterial puncture. Two subjects in the placebo group reported slight tingling sensation at the site of the puncture after the procedure.

Conclusion: Local AnGel is not effective in the reduction of pain during arterial puncture when applied for 30 minutes prior to the procedure. This is an important finding suggesting widespread use of local anaesthetic in this setting is not justified.

Key words: arterial puncture; local anaesthetic.
A COMPARISON OF VENTILATORY MODES OF NONINVASIVE POSITIVE PRESSURE VENTILATION IN ACUTE HYPERCAPNIC RESPIRATORY FAILURE IN CHRONIC OBSTRUCTIVE AIRWAYS DISEASE

Khoa Tran' ${ }^{1}$ Robyn O'Sullivan' ${ }^{1}$ Neil Bretz' ${ }^{1}$ and Elizabeth Voss ${ }^{2}$ Departments of Respiratory Medicine ${ }^{1}$ and Physiotherapy2 ${ }^{2}$ Greenslopes Private Hospital, 4120

Aim: The role of noninvasive positive pressure ventilation (NIPPV) in patients with chronic obstructive airways disease (COAD) presenting in acute hypercapnic respiratory failure (AHRF) is now well established. However, no studies have compared different ventilatory modes in this group. The aims of this study were to compare the efficacy of NIPPV in spontaneous (S) and spontaneous/timed $(\mathrm{S} / \mathrm{T})$ modes in AHRF complicating COAD, in the respiratory ward setting. Methods: Thirteen subjects presenting with COAD and AHRF, considered unsuitable for mechanical ventilation, were recruited into a double blinded trial with NIPPV via a full face mask randomised to either $\mathrm{S}$ or $\mathrm{S} / \mathrm{T}$ mode on a bi-level airway pressure device (Respironics BiPAP STD). Arterial blood gases (ABGs), respiratory rate (RR), Borg dyspnoea score (BDS) and pulse oximetry were measured at $0,1,6,24$, and 48 hours on NIPPV. Results: Baseline indices at time of randomisation were (mean \pm SD) age $76.6 \pm 6.3$ and $72.8 \pm 6.3$ years, FEV1 $0.45 \pm 0.13$ and $0.63 \pm 0.19$, RR $32.3 \pm 11.5$ and $28.0 \pm 5.7 \mathrm{bpm}$, BDS $9.0 \pm 2.0$ and $7.3 \pm 2.2, \mathrm{pH} 7.30 \pm 0.04$ and $7.27 \pm 0.06$, and $\mathrm{pCO}_{2} 70.3 \pm 11.5$ and $75.2 \pm 15.1 \mathrm{mmHg}$, in the $\mathrm{S}$ and $\mathrm{S} / \mathrm{T}$ groups respectively. There were no significant differences in indices between ventilatory modes at any time point. All indices improved significantly from baseline to 1 hour $(0<0.05)$ when groups were combined. There were no NIPPV induced complications. Conclusions: In a highly selected group of elderly subjects with COAD and AHRF, NIPPV significantly improved ABGs, RR and dyspnoea within 1 hour using either $S$ or $S / T$ modes. No significant difference between ventilatory modes was demonstrated which may reflect the small numbers, and recruitment is ongoing. NIPPV was effectively managed in a respiratory ward setting.

Key words: Noninvasive positive pressure ventilation, acute hypercapnic respiratory failure, BiPAP

\section{VALIDATION OF A SLEEP APNOEA SYMPTOM QUESTIONNAIRE}

Christopher Worsnop, Nicole Goh, Maree Barnes, Russell Goudge, Mark Howard.

\section{Department of Respiratory Medicine. Austin and Repatriation Medical} Centre, Victoria. 3084

Barnes et al. have assessed nasal CPAP in patients with mild obstructive sleep apnoea (OSA) and found that a sleep apnoea symptom questionnaire (SASQ) was the only test that showed a significant improvement with CPAP. Aim: To validate the SASQ with the following aims: (I) To establish the feasibility of answering the questionnaire. (II) To establish that SASQ scores are low in normal people. (III) To determine the difference in the scores between normal subjects and patients with OSA. (IV) To determine the correlation between the SASQ and ESS, RDI, arousal index (AI) and Maislin score (MAP). (V) To test the consistency of answering the questionnaire over time. (VI) To determine the difference in scores before and after treatment with CPAP in patients with OSA.

Methods: The normal group was medical students, who completed the SASQ ESS and MAP twice. The patient group was patients referred for a sleep study. They completed the questionnaires on 3 occasions, twice before and once after treatment with CPAP. The SASQ consisted of 14 items with responses given on a visual anaiogue scale 0 to $100 \mathrm{~mm}$. Lower scores meant fewer symptoms. Results: $\mathbf{N}=70$ students (29 females) and 106 patients ( 24 females). (I) $94 \%$ students and $86 \%$ patients successfully completed the SASQ. (II) The responses of the students were in the lower third of the range of possible scores. (III) Mean (sd) SASQ scores of patients with RDI $<5$ and RDI $\geq 5$ : 53.9 (22.4) and $69.0(22.0)(p=0.004)$. (IV) Correlation between SASQ and ESS $r=0.64, \mathrm{MAP} r=0.65, \mathrm{RDI} r=0.14$, and $\mathrm{Al} r=0.23(\mathrm{~V})$ Consistency of answering the SASQ: $r=0.93(\mathrm{VI})$ SASQ scores before and after CPAP were 70.7 (15.4) and $38.9(24.1)(p=0.001)$.

Conclusions: The SASQ was able to be completed by patients, produced low scores in normal people, had different scores between people with and without OSA, and had poor correlation with $\mathrm{RDI}$ and $\mathrm{Al}$, but good correlation with ESS and MAP. It was answered reliably and was responsive when OSA was treated. Key words: sleep apnoea, questionnaire. 
AN INVITRO ASSESSMENT OF NONINVASIVE BILEVEL POSITIVE PRESSURE VENTILATORS.

A Wright' ${ }^{1}, \mathrm{H}$ Niel2, A Ramsden², P Holmes' ${ }^{1}$ N Freezer'1. 1.Monash Medical Centre, Clayton, Victoria, Australia.2.Ritchie Centre, Monash University.

BACKGROUND: Noninvasive positive pressure ventilation (NIPPV) can effectively treat patients with acute respiratory failure. However, the performance of the NIPPV ventilators at high respiratory rates has not been described. AIM: To examine and compare the performance of NIPPV ventilators across a range of respiratory rates. METHOD: A spontaneously breathing respiratory model was built and connected, in turn, to 4 different ventilators capable of providing NIPPV. They are designated ventilator $A, B$, $C, D$. Each device was set up in a spontaneous mode to deliver, when triggered, an inspiratory pressure of $10 \mathrm{cmH}_{2} \mathrm{O}$ and an expiratory pressure of $5 \mathrm{cmH}_{2} \mathrm{O}$. The respiratory model breathed at respiratory rates between 10 and 50 breaths per minute. The respiratory model was similar to a normal thorax, having a compliance of $100 \mathrm{ml} / \mathrm{cmH}_{2} \mathrm{O}$ and a resistance of 2.4 $\mathrm{cmH}_{2} \mathrm{O}$.sec.L-1, but different in that the inspiratory to expiratory rate was fixed at a ratio of $1: 1$ and that both phases of respiration were active. RESULTS: Table 1. Average inspiratory and expiratory pressures.

\begin{tabular}{|c|c|c|c|c|c|}
\hline Resp. rate & 10 & 20 & 30 & 40 & 50 \\
\hline A : Insp. & 14 & 13.4 & 12.7 & 11.9 & 10.3 \\
\hline Exp. & 9.7 & 9.8 & 9.9 & 10.3 & 11.8 \\
\hline B : Insp. & 8.9 & 8.3 & 8.3 & 7.4 & 6.2 \\
\hline Exp. & 6.2 & 6.3 & 6.6 & 7.1 & 7.6 \\
\hline C:Insp. & 10.1 & 9.1 & 7.7 & 6.3 & 4.7 \\
\hline Exp & 5.8 & 6.1 & 6.7 & 7.4 & 8.4 \\
\hline D:Insp. & 8.4 & 8.1 & 6.8 & 5.2 & 3 \\
\hline Exp. & 4.6 & 5.6 & 6.4 & 7.5 & 9 \\
\hline
\end{tabular}

CONCLUSION: As the respiratory rate increased beyond 30 breaths $/ \mathrm{min}$. the devices failed to deliver effective bilevel ventilation.

Key words: Noninvasive ventilation, ventilator performance, respiratory model.

\section{BRONCHOSCOPY IN FEBRILE NEUTROPENIC PATIENTS WITH} PULMONARY INFILTRATES.

Mylinh Duong', Sharon Morton²

We undertook a retrospective review of 35 (20 men, 15 women) consecutive neutropenic patients with pulmonary infiltrates who failed to respond to conventional neutropenic antibiotic protocol. These patients were referred for bronchoscopy with washings, brushings, lavages and bronchial biopsies obtained. The age range was 17-77 years, with the commonest cause for neutropenia being chemotherapy. The mean duration of neutropenia and antibiotic therapy was 14 days ( $2-40$ days) and 11 days ( $1-40$ days) respectively. The yield from all types of specimens was low. The extent of the radiological involvement did not influence the yield. The commonest results obtained were growth of bacteria and fungi which were difficult to interpret without a histological correlation. The procedure overall impacted on the management of 8 patients. It was useful in excluding an infective cause and allowing rationalization of antimicrobial therapy in non critically ill patients but was disappointing in aiding the management of the critically ill where mortality remained high. However, the procedure was safe with a low complication rate despite the presence of hypoxaemia and thrombocytopenia. Open lung biopsy provided a diagnosis of pneumocystis in 2 cases when bronchoscopy was negative. In conclusion, bronchoscopy in this setting proved to be disappointing even with lavage specimens in identifying an infectious cause. Perhaps in conjunction with the routine use of transbronchial biopsies, we can improve the yield of this procedure and render it a useful diagnostic tool in the management of these patients without the elevated risk of complications which often accompanies other invasive pulmonary procedures.

Key words: Neutropenia, bronchoscopy, pulmonary infiltrates

\section{A PROTOCOL FOR THE MANAGEMENT OF COMMUNITY ACQUIRED PNEUMONIA IS COST-EFFECTIVE \\ C. Dobbin \\ Department of Respiratory Medicine. Royal Prince Alfred Hospital, NSW $\underline{2050}$}

In July 1998 a district hospital (DH) implemented a protocol for the treatment of community acquired pneumonia (CAP) in order to eliminate empirical use of third generation cephalosporins (TGC). This study assesses its efficacy and cost-effectiveness by comparing patient outcomes with those of patients treated at an urban teaching hospital $(\mathrm{UH})$ where standard theraby for CAP involves the use of TGC. Methods: A retrospective review of patients with coded records admitted to the $\mathrm{DH}$ and $\mathrm{UH}$ with CAP between January 1999 and June 1999 was performed. Rate of compliance with the protocol by emergency department (ED) staff at the DH was assessed. The age, sex, severity, smoking history, underlying lung disease, initial treatment, culture results, duration of fever and intravenous (IV) therapy and length of stay (LOS) were compared and an estimate of cost effectiveness of the protocol made. Results: Of the 86 patients with CAP at the DH $52(60 \%)$ received treatment as per protocol. Twenty-four patients (28\%) received empirical treatment with TGC despite the protocol. Of the 62 initially treated with penicillin or ampicillin, $8(13 \%)$ were later treated with TGC because of failure to improve. Protocol adherence was significantly less likely in patients allergic to penicillin (RR $0.35 ; 95 \% \mathrm{Cl} 0.16$ to $0.76)$. At the UH $93 \%$ (67/72) of patients with CAP received empirical treatment with TGC. Patients at the DH were older (63.0 vs 56.6 years; $p=$ 0.04 ) and on more regular medications $(4.0 \mathrm{vs} 2.6, p=0.002$ ). The vital signs, white cell counts, creatinine levels, prevalence of underlying lung disease, intercurrent illness, smoking history and rate of clinically relevant culture results were similar in the two groups. There was no significant difference in time to defervescence $(p=0.4)$ or LOS $(p=0.62)$. Mean duration of IV therapy did not differ significantly ( 4.2 days $\mathrm{DH}$ vs 4.9 days $\mathrm{UH} ; \mathrm{p}=0.14$ ). Ampicillin treatment for one day costs $\$ 4.40$ ( $\$ 1.10 \times 4$ ) and ceftriaxone $\$ 19.38$. Therefore, the DH saves an average of $\$ 77.73$ for every patient treated as per protocol.

Conclusion: One year after implementation, a protocol for the treatment of CAP is proving efficacious and cost-effective. Compliance with the protocol by ED staff is good but could be improved by continuing education.

Key words: Pneumonia, third generation cephalosporins, cost-effectiveness.

\section{TUBERCULOSIS UNDERTAKING CLINIC - A 27 MONTH EXPERIENCE}

Michael Fiynn, Harry Teichtahl Department of Respiratory Medicine. Western Hospital. Footscray. Victoria

Background: Subjects migrating to Australia as permanent residents or entering Australia as students are required to have a health screen provided by the Department of Immigration and Multicultural Affairs. Those found to have abnormal chest $X$-rays (CXR) which are not due to active TB, sign a Tuberculosis Undertaking (TBU) and are reviewed when in Australia. Aim: To review the experience of a TBU clinic and outcomes of refecral from the TBU clinic for isoniazid preventive therapy (IPT). Methods: Prospective analysis of CXR and Mantoux test results and retrospective analysis of TBU clinic patients referred for IPT. Results: Between November 1996 and February 1999, 2395 subjects were referred to the TBU Clinic, of whom $1919(80 \%)$ attended in person and $203(8 \%)$ had X-ray only review. The most common countries of birth were China (20\%), Vietnam (12\%), India (6\%) and Philippines $(6 \%)$. Assessment was: inactive TB (39\%), possibly active TB (1.6\%), non-TB (12\%), or normal (47\%). Twenty-eight percent of TBU subjects were referred to a consultant, $13 \%$ to a general practitioner, and $58 \%$ were discharged without follow-up. Mantoux ( $10 \mathrm{TU}$ ) was $\geq 15 \mathrm{~mm}$ in 106 of $272(39 \%$ ) subjects aged $<35$ years who had an abnormal CXR or were from a high risk country. Of 126 people referred for IPT, $9(7 \%)$ did not attend their appointment at Western Hospital TB clinic and $13(10 \%)$ were referred to other clinics. Of the 104 who were followed at Western Hospital, IPT was offered to $78(75 \%)$. Of these, 9 (12\%) refused IPT, 20 (26\%) started but failed to complete IPT and $49(63 \%)$ completed six months IPT. Conclusions: Of subjects referred on a TBU, $47 \%$ had no significant pulmonary abnormality on expert review. Forty-three percent of TBU subjects referred for IPT and managed at Western Hospital completed 6 months therapy.

Support: Department of Human Services, Victoria. Key words: tubercuiosis, migrants, isoniazid preventive therapy. Nominations for awards: nil. 


\section{FACTORS INFLUENCING PRESCRIPTION OF ISONIAZID CHEMO-} PREVENTIVE THERAPY

Hart DHL.

Department of Respiratory Medicine, St Vincent's Hospital, Melbourne

Background: Physicians vary widely in their use of isoniazid (INH) chemopreventive therapy in subjects with a positive Mantoux reaction. In this study a questionnaire was used to ascertain the reasons physicians prescribed or withheld INH in two groups of subjects. Method: 18 physicians seeing 181 health care workers detected by hospital staff screening, and 103 migrants in a migrant screening clinic, all with Mantoux $>15 \mathrm{~mm}$, filled a questionnaire for each patient detailing their reasons for or against use of INH. Results: 79 of the 181 health care workers were <35yrs mean Mantoux size $20 \mathrm{~mm}$; (range 15-58), 10 were recommended INH, 5 agreed to take it. Of the 69 not offered INH physicians recorded the following reasons: known past positive Mantoux 6, reactivation unlikely 11, Mantoux likely to be due to past BCG 46, others 6 . Among 103 migrants (mean age $30.7 y$ rs, range 16-71), mean Mantoux size 20.7, (range 15-50), 76 were offered INH, 67 accepted. Of the 27 not offered INH physician reasons were as follows: previous adequate treatment 7 , reactivation unlikely 14 , Mantoux likely to be due to prior BCG 2, others 4 .

Conclusions: Prescription of INH chemo-preventive therapy is highly context sensitive and not driven by size of Mantoux alone. Simple prescription algorithms are unlikely to be of use. Physicians more often ascribed Mantoux positivity to prior BCG vaccination in health care workers of many ethnic backgrounds than they did in migrants who had also had high rates of prior BCG.

Key words: isoniazid, tuberculosis, chemo-preventive therapy

SUCCESSFUL OUTCOME FOR NON-HIV MULTIDRUG RESISTANT TUBERCULOSIS (MDR TB) TREATED WITH DIRECTLY OBSERVED THERAPY (DOT).

Seeto, L.J., Erdstein, A.A., Lagaac, E.M., Breslin, A.B.X. Respiratory Unit, Concord Hospital, Sydney, AUSTRALIA.

Aim: To review the experience in non-HIV MDR TB individuals.

Methods: Five consecutive patients with positive sputum smears \& MDR cultures are presented.

\begin{tabular}{|l|l|l|l|l|}
\hline $\begin{array}{l}\text { Patient } \\
\text { (Pt) }\end{array}$ & $\begin{array}{l}\text { Sex/Age } \\
\text { (years) }\end{array}$ & $\begin{array}{l}\text { Country of } \\
\text { birth }\end{array}$ & $\begin{array}{l}\text { Time in } \\
\text { Australia }\end{array}$ & Chest radiograph \\
\hline 1 & F 36 & Philippines & 5 years & RU \& RLL cavities \\
\hline 2 & M 28 & Korea & 6 months & LUL cavity \\
\hline 3 & M 34 & Korea & 2 years & $\begin{array}{l}\text { RUL, RMZ \& LUL } \\
\text { infiltrate }\end{array}$ \\
\hline 4 & M 45 & U. K. & 35 years & Bilateral cavities \\
\hline 5 & M 14 & China & 2 years & LUL infiltrate \\
\hline
\end{tabular}

All patients were given DOT with 5 to 8 drugs, chosen from: ethambutol, pyrazinamide, ciprofloxacin, amikacin, capreomycin, clofazamine, cycloserine, para-aminosalicylic acid, amoxycillin/clavulanate, interferon $\gamma$. Two patients came to surgery for cavities: Pt 1 - right upper lobectomy, Pt 4 - right upper \& middle \& left upper lobectomies. Results: Duration of followup from $O$ (current treatment) to 6 years. Patients $1,2 \& 3$ completed treatment \& remain smear \& culture negative with improved chest radiographs. Patients $4 \& 5$ are still on therapy. Pt 4 had most of the disease removed and became smear negative 14 weeks into treatment. Pt 5 is now smear negative with marginal radiographic improvement.

Conclusion: A successful outcome in these 5 patients has been achieved. Two patients still continuing treatment show signs of recovery. Aggressive treatment with at least 5 drugs combined with surgery in cavitatory disease gives promise of cure in this difficult, life-threatening disease.
PULMONARY MANIFESTATIONS OF MALARIA

Graeme Maguire1, Susan Jacups², Dale Fisher'2, Tim Cain ${ }^{3}$, Paul Marks², Bart Currie1.2, Nick Anstey1,2

(1) Menzies School of Health Research. Darwin 0810 (2) Flinders University, Royal Darwin Hospital Clinical School (3) NT Imaging

Severe pulmonary disease is an infrequent complication of malaria. Clinical manifestations of milder disease however occur in up to $18 \%$ of patients with Plasmodium falciparum infection with a reduction in PEFR on the third to fourth day of symptoms'. Methods: prospective study of 10 subjects returning from malaria endemic regions who were hospitalised with blood smear confirmed malaria. Lung function testing was performed daily for 1-5 days, and at 1 week post discharge. Lung function testing included spirometry, single breath $\mathrm{CO}$ diffusion (DL $\mathrm{CO}_{\mathrm{CO}} \mathrm{SB}$ ), body plethysmograph lung volumes, and airway resistance. CXRs were performed on day $1 \& 3$ and 1 week after discharge, $99 \mathrm{mTc}$-DTPA ventilation scans on day 1,3,5 and 89mTc-sulphur-colloid scans on day 1,2 and 3 Results: $3 / 10$ subjects had cough on the day of presentation. Cough was more common in subjects with $P$. vivax (3/4) compared with those subjects with $P$. falciparum (0/6) (Fisher's exact, $p=0.01$ ) This difference persisted until at least day 4 postpresentation. Predicted FEV 1 had a mean increase of $4.5 \%$ $(1.9(\mathrm{SEM}))$ from day 1 to day 5 post-presentation and DLCOSB

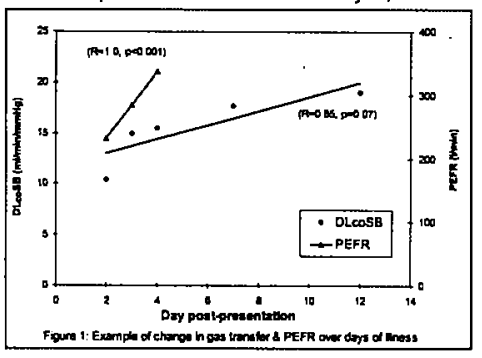
$11.5 \%$ (4.2(SEM)). Conclusion: Mild pulmonary involvement is common in patients with malaria. Increased investigation of respiratory physiology in this condition may facilitate a greater understanding of what is a multisystem disease.

Supported by: Flinders University Northern Territory Clinical School Key words: Malaria, respiratory physiology, nuclear medicine

Nominations for Awards: nil

1. Gozal, D. The incidence of pulmonary manifestations during Plasmodium falciparum malaria in non immune subjects. 1992 Trop Med Parasitol; 70: 6-8.

IN VITRO EFFECTS OF STREPTOKINASE AND DEOXYRIBONUCLEASE ON VISCOSITY OF HUMAN SURGICAL AND EMPYEMA PUS

Graham Simpson, David Roomes, Mal Heron

Purified streptokinase is now frequently used intra-pleurally to treat empyema thoracis replacing the older preparation (Varidase ${ }^{T M}$ ) which contains streptococcal DNase as well as streptokinase. Our clinical impression was that the purified preparation was less effective than Varidase. To investigate whether DNase was contributing to easier drainage of pus we have measured pus viscosity using a simple viscosometer. Pus from three soft issue abscesses drained surgically and from six patients with empyema thoracis was studied. Pus samples were incubated with normal saline (as control) and with streptokinase, Varidase, human recombinant DNase (Pulmozyme ${ }^{T M}$ ) and a mixture of streptokinase and Pulmozyme.

Purified streptokinase had little effect on pus viscosity with mean reduction of $11.1 \%$ in surgical specimens and $1.7 \%$ in empyema samples. Varidase reduced viscosity by a mean of $52.8 \%$ in surgical samples and $94.8 \%$ in empyema samples and human recombinant DNase reduced viscosity by a mean of $32.8 \%$ in surgical samples and $93.4 \%$ in empyema samples. Adding streptokinase to DNase did not reduce viscosity further. Final viscosities in all samples treated with DNase were similar whatever the starting viscosity.

DNase thus significantly reduces pus viscosity whereas streptokinase has little effect and may work simply by breaking down loculations in empyema thoracis. Combining streptokinase with DNase may be of clinical benefit in empyemas where pus viscosity is high 


\section{SUCCESSFUL TREATMENT OF EMPYEMA THORACIS} WITH HUMAN RECOMBINANT DNA

\author{
Graham Simpson and Ben Reeves
}

Treatment of empyema thoracis with fibrinolytic enzymes if simple tube drainage fails is increasing in popularity. Recent work $\mathbf{1 . 2}$ suggests that streptokinase may break down fibrinous loculations in empyemas but has no effect on pus viscosity whereas DNase does reduce pus viscosity and may facilitate drainage.

An 82 year old lady presented with pneumococcal pneumonia and subsequently developed an empyema. Treatment with intravenous antibiotics and intercostal tube drainage for five days failed to improve the empyema and a second intercostal drain was inserted. Streptokinase in standard doses (250,000U daily for 3 days) was instilled into the pleural cavity but the empyema persisted. The patient refused surgical intervention. She was treated with intrapleural human recombinant DNase $5 \mathrm{mg}$ daily for 3 days. This resulted in drainage of further $700 \mathrm{ml}$ of pus with clinical and radiological improvement.

We believe this is the first use of human recombinant DNase in treating empyema thoracis in man.

References:

1) Simpson G., Roomes D. Thrombolytics and pleural empyema Medical Journal of Australia. 1998. 168, 144

2) Simpson G., Roomes D. Heron M. Effects of streptokinase and deoxyribonuclease on viscosity of human surgical and empyema pus. Thorax (in press)

\section{SPUTUM INDUCTION IN A HETEROGENEOUS POPULATION.}

Alistair Wright, Peter Holmes, Department of Respiratory Medicine, Monash Medical Centre Clayton, Victoria, Australia, 3168.

BACKGROUND: Inhaled hypertonic saline via an ultrasonic nebuliser can be effectively used to induce coughing and sputum production for the diagnosis of diseases of the lower respiratory tract. However the place of this technique in populations with ready access to fibre-optic bronchoscopy (FOB) remains controversial. AlM: To compare sputum induction with FOB in the assessment of patients with lower respiratory tract diseases. METHOD: Induced sputum samples (20mls of $6 \%$ saline given as continuous nebuliser) were taken from 16 consecutive patients referred to the primary investigator with one or more of the following two indications for FOB: (a) haemoptysis for investigation (in patients age $>40$ yo, a smoking history and an abnormal $\mathrm{CXR}$ ) and/ or (b) possible TB (with a significant clinical risk of TB and negative sputum smears). All induced sputum procedures were well tolerated with no adverse effects seen. All patients proceeded on the same day to FOB. The type of specimen taken at FOB was at the discretion of the bronchoscopist. Specimens from both investigations were sent to the same laboratory and were analysed with cytology and microscopy and culture, including AFB. RESULTS: Adequate induced sputum specimens were obtained in 11 of the 16 patients. The FOB included general washings $(n=15)$, brushings $(n=10)$, lavage $(n=5)$, biopsy $(n=5)$. In total 8 patients received definitive diagnoses. 5 malignancies ( 2 small cell, 2 adenocarcinomas and 1 squamous cell lung cancer), MTB was diagnosed in two patients and an atypical TB was diagnosed in one patient. 7 of these 8 patients with diagnoses had adequate induced sputum specimens. Bronchoscopy diagnosed all 8 cases while the induced sputum technique diagnosed oniy 2 of the malignancies also seen on bronchoscopy (odds ratio in favour of bronchoscopy $=7: 1$ ). CONCLUSION: In our small, heterogeneous group of patients, sputum induction did not provide additional diagnoses to $\mathrm{FOB}$

Key words: Fibre-optic bronchoscopy, sputum induction.

\section{TRANS-THORACIC NEEDLE ASPIRATION IN COMMUNITY-ACQUIRED} PNEUMONIA.

William Slater ${ }^{1}$, Graham Mills ${ }^{1,2}$. Noel'Karalus' ${ }^{1}$, Clare Coles ${ }^{2}$, Yvonne Kuys ${ }^{2}$ \& Ray Cursons ${ }^{3}$.

(1) Department of Respiratory Medicine, and (2) Department of Respiratory Research Services, Waikato Hospital, Hamilton, New Zealand, and (3) Department of Biological Sciencess, Waikato University, Hamilton. New Zealand.

In New Zealand and Australia trans-thoracic needle aspiration (TNA) is not a commonly used method of obtaining diagnostic material in communityacquired pneumonia (CAP). Conventional culture techniques usually require more than $24 \mathrm{hrs}$ to yield a microbiologicai diagnosis. Molecular techniques have recently been shown to increase the diagnostic yield by $33 \%$ to $44 \%$. Pneumothorax is the most common complication and has been reported in up to $30 \%$ of procedures. There is variation in the use of radiological guidance for TNA. Methods: Prospective study of all patients admitted to our hospital with CAP. TNA was performed on patients in whom there were no contraindications and from whom informed consent was obtained. For TNA to proceed certain chest ultrasound features were required. The complication rate of TNA and the patient's perceptions of the procedure were recorded. Results: 84 patients have been enrolled to date. 18 patients were asked to undergo TNA, 13 patients consented, 3 did not meet the ultrasound requirement, 10 patients had TNA. There were no pneumothoraces, 4 patients experienced pain during the procedure, and 1 patient would not have had a repeat procedure. Conclusions: TNA is not associated with an unacceptable complication rate and patients find it an acceptable procedure. TNA in combination with molecular techniques may improve the accuracy and reduce the time to microbiological diagnosis.

Supported by Respiratory Research Services, Waikato Hospital Key words: pneumonia, trans-thoracic needle aspiration, complications.

* Refer to page A16 for Pa10A 